1157

ILLINOIS INSTITUTE OF TECHNOLOGY

THIS DOCUMENT CONFIRMEO AS UNCLASSIFIED

DIVISION OF CLASSIFICATION.

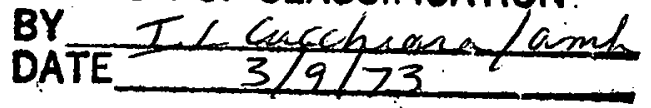

\title{
MICROMECHANICS ANALYSIS OF COMPOSITE MATERIALS \\ USING FINITE ELEMENT METHODS
}

BY

BHAGWAN DASS AGARWAL

Submitted in Partial Fulfillment of the

Requirements for the Degree of

Doctor of Philosophy in Mechanics

in the Graduate School of

Illinois Institute of Technology

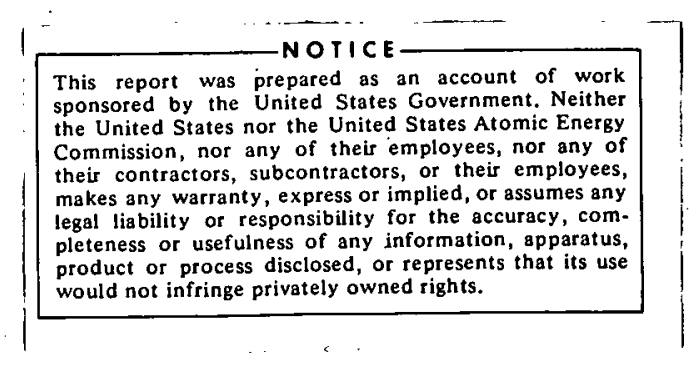

Approved

CHICAGO, ILLINOIS

May 1972

Adviser

DISTRIEUTISN OF THIS DOCUAFNT IS URYAMITED 


\section{DISCLAIMER}

This report was prepared as an account of work sponsored by an agency of the United States Government. Neither the United States Government nor any agency Thereof, nor any of their employees, makes any warranty, express or implied, or assumes any legal liability or responsibility for the accuracy, completeness, or usefulness of any information, apparatus, product, or process disclosed, or represents that its use would not infringe privately owned rights. Reference herein to any specific commercial product, process, or service by trade name, trademark, manufacturer, or otherwise does not necessarily constitute or imply its endorsement, recommendation, or favoring by the United States Government or any agency thereof. The views and opinions of authors expressed herein do not necessarily state or reflect those of the United States Government or any agency thereof. 


\section{DISCLAIMER}

Portions of this document may be illegible in electronic image products. Images are produced from the best available original document. 


\section{PREFACE}

Finite element methods have been used to calculate internal stresses within a spherical particle and discontinuous fiber composite. The internal stresses have been analyzed as a function of interparticle spacing and the elastic constants have been calculated from a knowledge of these stresses. The effect of altering the interface properties has been simulated by assigning different properties to the finite elements forming the boundary of the interface.

The author expresses his sincere thanks and gratitude to Professor Lawrence J. Broutman for accepting the supervision of this work and highly appreciates his most valuable discussions and suggestions. Without the continuous help and personal attention of Dr. Broutman it would not have been possible for the author to complete the research work. The author is indebted to Dr. Chiarulli for his timely and most needed help at the beginning of this work. Thanks are due to Doctors R. C. Dix and G. A. Panizza for their help with the computer program for the axisymmetric analysis and to Dr. R. L. Chiapetta for his help with the program SAFE-3D. Dr. J. M. Lifshitz deserves special mention for his very helpful discussions and suggestions. The author appreciates Messrs. S. K. Gaggar, P. K. Mallick, S. M. Krishnakumar and T. Kobayashi for providing a lively atmosphere conducive to the research work in the Mechanics of Materials Laboratory. The author wishes to express thanks to his friends Sudhari Sahu and Pratap Singh Surana for overall encouragement in the research work. Finally, the author thanks Dewki and Kiran Agarwalla for the illustrations in the thesis and Mrs. Judy Masilun for carefully typing the thesis.

The research work described here was supported by the Atomic Energy Commission under contract No. AT(11-1)-1794. 
PREFACE .................... ii

LIST OF TABLES $\ldots \ldots \ldots \ldots \ldots \ldots \ldots \ldots$

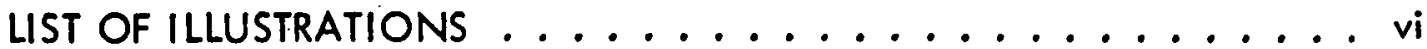

\section{SECTIONS}

1. INTRODUCTION .................... I

11. AXISYMMETRIC ANALYSIS OF PARTICULATE COMPOSITES .. 5

Introduction

Approximations and Boundary Conditions

Internal Stresses

Elastic Constants

Effect of a Weak Interface on Composite Properties

III. THREE-DIMENSIONAL ANALYSIS OF PARTICULATE

COMPOSITES ............................. 37

Introduction

Boundary Conditions

Internal Stresses

Elastic Constants

IV. ELASTIC ANALYSIS OF THREE PHASE FIBROUS COMPOSITES . 58

Introduction

Representative Area

Boundary Conditions and Component Properties

Stresses

Effect of Interface

Effect of Fiber End Condition

Some Comments on the Results

V. ELASTIC-PLASTIC ANALYSIS OF FIBROUS COMPOSITES $\ldots .94$

Introduction

Analysis of the Models

Boundary Conditions

Stresses with Axial Strains Imposed on One Boundary of the Representative Cell

Stress-Strain Curves with Axial Strains Imposed on One Boundary of the Representative Cell

Stresses and Stress-Strain Curves with Axial Strains

Imposed on Entire Outer Boundary of the

Representative Cell 


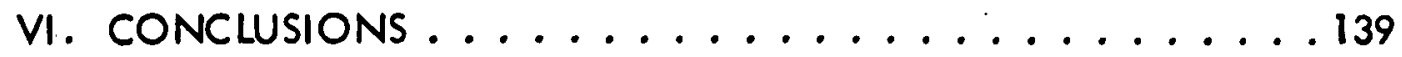

APPENDIX ................................

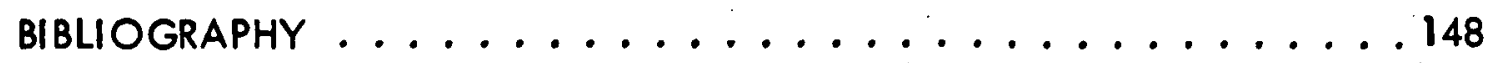




\section{LIST OF TABLES}

Table

1. The Relation Between Particle Spacing and Volume Fraction ....... 17

2. Predicted Properties of Porous and Filled Ceramics ............... 30

3. Effect of Interface on Modulus of Elastic ity of Composite :......... 36

4. Predicted Modulus of Elasticity of Alumina Filled Glass ......... 55

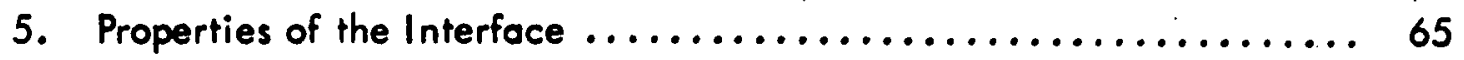

6. Average Composite Stresses for $\frac{r_{2}}{r_{1}}=0.67$ and $\left(\sigma_{y}\right)_{m}=8,000$ psi $\ldots . .106$

7. Average Composite Stresses for $\frac{r_{2}}{r_{1}}=0.67$ and $\left(\sigma_{y}\right)_{m}=20,000$ psi $\ldots 108$

8. Average Composite Stresses for $\frac{r_{2}}{r_{1}}=0.25$ and $\left(\sigma_{y}\right)_{m}=8,000$ psi $\ldots \ldots 114$

9. Average Composite Stresses for $\frac{r_{2}}{r_{1}}=0.67$ and $\left(\sigma_{y}\right)_{m}=8,000$ psi .... 129 


\section{LIST OF ILLUSTRATIONS}

Figure

Page

1. Unit Cell for Axisymmetric Representation of Sphere-Filled Composite

2. Finite Element Grid for $r_{2}{ }^{1} r_{1}=0.357 \ldots \ldots \ldots \ldots \ldots \ldots \ldots . . \ldots$

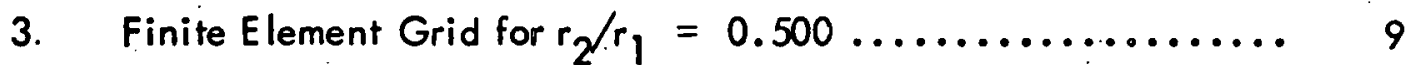

4: Finite Element Grid for $r_{2} / r_{1}=0.615 \ldots \ldots \ldots \ldots \ldots \ldots \ldots$

5. Finite Element Grid for $r_{2} / r_{1}=0.714 \ldots \ldots \ldots \ldots \ldots \ldots \ldots$

6: Finite. Element Grid for $r_{2} / r_{1}=0.833 \ldots \ldots \ldots \ldots \ldots \ldots \ldots$

7. Finite Element Grid for $r_{2} / r_{1}=0.870 \ldots \ldots \ldots \ldots \ldots \ldots \ldots$

8. Volume Packing (A) Sphere in a cylinder, (B) Hexagonal Array, and $(C)$ Square or Cubic Array ....................... 16

9. Matrix Stresses at Pore Boundary in Porous Ceramic with

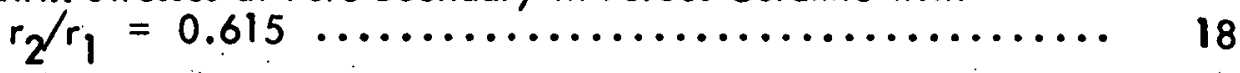

10. Matrix Stresses at Pore Boundary in Porous Ceramic with

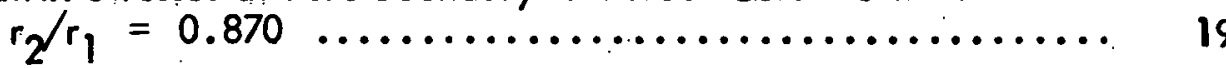

11. Pore Boundary Stresses as a Function of Porosity (vol. \%) ..... 20

12. Matrix Stresses in the Composite ................. 22

13. Matrix Stresses at Interface in Alumina-Filled Glass with

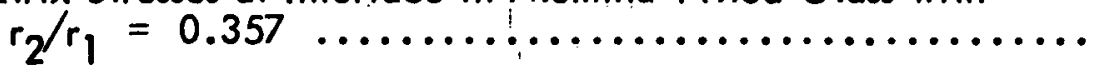

14. Matrix Stresses at Interface in Alumina-Filled Glass with

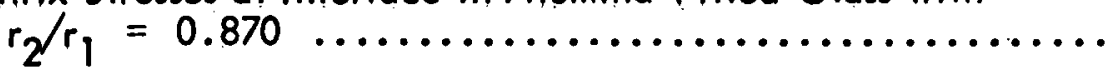

15. Interfacial Stresses in Alumina-Filled Glass as a Function of Volume Fraction of Alumina $\ldots \ldots \ldots \ldots \ldots \ldots \ldots$.

16. Comparison of Predicted Modulus of Elasticity of Porous Ceramic with Experimental Data and Prediction of Poisson's Ratio ...

17. Comparison of Predicted Modulus of Elasticity of Alumina-Filled Glass with Experimental Data of Reference $14 \ldots . . . . .$. .

18. Comparison of Predicted Modulus of Elasticity of Tungsten-Filled Glass with Experimental Data of Reference 15 
19. Shaded Elements Represent Finite Thickness of the Interface

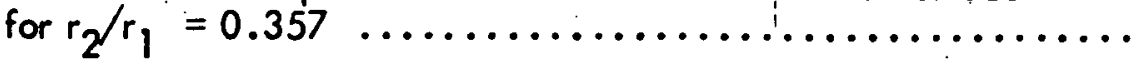

20. Shaded Elements Represent Finite Thickness of the Interface for $r_{2} / r_{1}=0.714$

21. Comparison of Matrix Stresses With and Without a Weak Interface

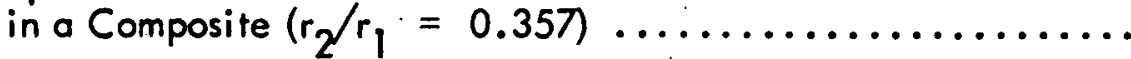

22. Comparison of Matrix Stresses With and Without a Weak Interface

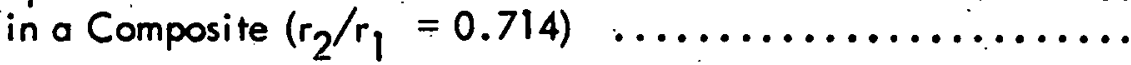

23. Finite Element Types: (A) Tetrahedral Element, (B) Membrane Plane Element, and (C) Uniaxial Bar Element ...........

24. Octahedral Element and Degenerate Cases: (A) Basic Octahedral Element, (B) Two Tetrahedra Degenerate Case, and (C) One Tetrahedron Degenerate Case

25. Packing of Spherical Inclusions in a Composite .......... 41

26. A Typical Three-Dimensional Region Analyzed ........... 42

27. Three-Dimensional Mesh for $r_{2} / r_{1}=0.357 \ldots \ldots \ldots \ldots \ldots \ldots 43$

28. Three-Dimensional Mesh for $r_{2} / r_{1}=0.714 \ldots \ldots \ldots \ldots \ldots \ldots 44$

29. Three-Dimensional Mesh for $r_{2} / r_{1}=0.870 \ldots \ldots \ldots \ldots \ldots \ldots$

30. Three-Dimensional Mesh for $r_{2} / r_{1}=0.952 \ldots \ldots \ldots \ldots \ldots \ldots 46$

31. Matrix Stresses at Interface in Alumina-Filled Glass with

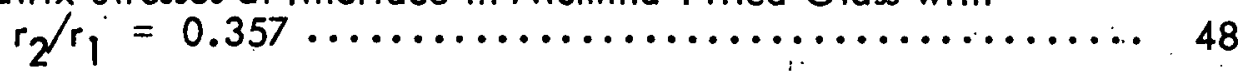

32. Matrix Stresses at Interface in Alumina-Filled Glass with $r_{2} / r_{1}=0.714$

33. Matrix Stresses at Interface in Alumina-Filled Glass with

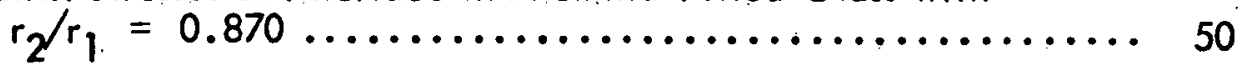

34. Matrix Stresses at Interface in Alumina-Filled Glass with

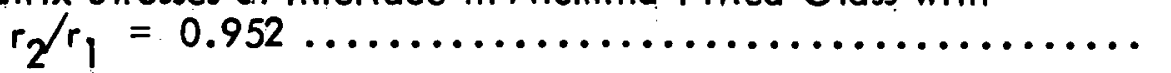

35. Interfacial Stresses in Alumina-Filled Glass as a Function

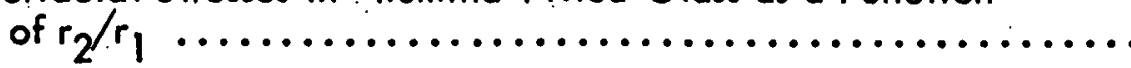

36. Comparison of Modulus of Elasticity of Alumina-Filled Glass as a Function of $r_{2} / r_{1}$, Predicted by Three-Dimensional and Axisymmetric Analyses 
37. Comparison of Modulus of Elasticity of Alumina-Filled Glass as a Function of Alumina Contents, Predicted by ThreeDimensional and Axisymmetric Analyses ............. 56

38. Symmetrical Packing of Discontinuous Fibers in a Composite ..... 61

39. Cell for Axisymmetric Representation of Fibrous Composite ...... 62

40. (A) Finite Element Grid for $r_{2} / r_{2}=0.67$, and (B) Finite Thickness of the Interface Represented by Shaded Elements .........6 63

41. Normalized Fiber Axial Stresses and Interfacial Shear Stresses Along Fiber Axis in a Two-Phase Composite ..........67

42. Radial Variation of Normalized Axial Stresses in the Matrix of a Two-Phase Composite ...................... 68

43. Normalized Matrix Axial Stresses Along Fiber Axis in a Two-Phase Composite .......................... 69

44. Normalized Matrix Radial Stresses Along Fiber Axis in a Two-Phase Composite ...................... 70

45. Normalized Fiber Axial Stresses Along Fiber Axis in a Three-Phase Composite ....................... 73

46. Normalized Interfacial Shear Stresses Along Fiber Axis in a Three-Phase Composite ....................... 74

47. Equilibrium of Infinitesimal Fiber Length $\ldots \ldots \ldots \ldots \ldots \ldots$

48. Normalized Axial Stress at the Fiber End as a Function of Interface Modulus ......................... 7

49. Radi al Variation of Axial Stresses Near Fiber End in a Three-Phase Composite. ..................... 79

50. Normalized Axial Stresses Along Fiber Axis in a Three-Phase Composite ....................... 80

51. Normalized Matrix Radial Stress Along Fiber Axis in a Three-Phase Composite ....................... 81

52. Composite Modulus as a Function of Interface Modulus ........ 83

53. Maximum Distortion Energy as a Function of Interface Modulus ... 85

54. Composite Strength as a Function of Interface Modulus (Qualitative Representation) 
55. Normalized Fiber Axial Stresses Along Fiber Axis for Different

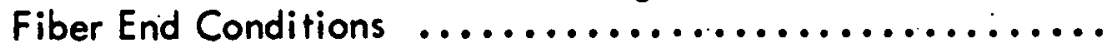

56. Normalized Interfacial Shear Stresses Along Fiber Axis for Different Fiber End Conditions ...................

57. Radial Variation of Axial Stresses Near Fiber End for Different

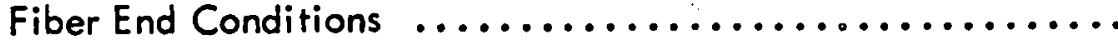

58. Normalized Axial Stresses Along Fiber Axis for Different Fiber End Conditions ....................... 9

59. Packing of Discontinuous Fibers in a Composite .......... 97

60. Finite Element Grids for $(A) r_{2} / r_{1}=0.67,(B) r_{2} / r_{1}=0.25 \ldots .98$

61. Fiber Axial Stresses Along Fiber Axis $\left(r_{2} / r_{1}=0.67\right.$, Matrix Yield Strain $=2.0 \%$, Axial Strain Imposed on One Boundary)

62. Interfacial Shear Stresses Along Fiber Axis $\left(r_{2} / r_{1}=0.67\right.$, Matrix Yield Strain $=2.0 \%$, Axial Strain Imposed on One Boundary)

63. Matrix Axial Stresses Along Fiber Axis $\left(r_{2} / r_{1}=0.67\right.$, Matrix Yield Strain $=2.0 \%$, Axial Strain Imposed on One Boundary)

64. Radial Variation of Matrix Axial Stress Near Fiber End $\left(r_{2} / r_{1}=0.67\right.$, Matrix Yield Strain $=2.0 \%$, Axial Strain Imposed on One Boundary) $\ldots \ldots \ldots \ldots \ldots \ldots \ldots \ldots \ldots \ldots$

65. Yielding of Matrix at Different Composite Strains $\left(r_{2} / r_{1}=0.67\right.$, Matrix Yield Strain $=2.0 \%$, Axial Strain Imposed on One

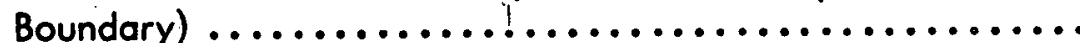

66. Fiber Axial Stresses Along Fiber Axis $\left(r_{2} / r_{1}=0.67\right.$, Matrix Yield Strain $=5.0 \%$, Axial Strain Imposed on One Boundary)

67. Interfacial Shear Stresses Along Fiber Axis $\left(r_{2} / r_{1}=0.67\right.$, Matrix Yield Strain $=5.0 \%$, Axial Strain Tmposed on

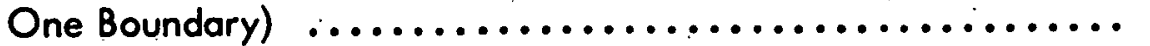

68. Matrix Axial Stresses Along Fiber Axis $\left(r_{2} / r_{1}=0.67\right.$, Matrix Yield Strain $=5.0 \%$, Axial Strain Imposed on One Boundary)

69. Radial Variation of Matrix Axial Stresses Near Fiber End $\left(r_{2} / r_{1}=0.67\right.$, Matrix Yield Strain $=5.0 \%$, Axial Strain Imposed on One Boundary) 
70. Yielding of Matrix at Different Composite Strains $\left(r_{2} / r_{1}=0.67\right.$, Matrix Yield Strain $=5.0 \%$, Axial Strain Imposed on One Boundary)

71. Fiber Axial Stresses Along Fiber Axis $\left(r_{2} / r_{1}=0.25\right.$, Matrix Yield Strain $=2.0 \%$, Axial Strain Imposed on One Boundary)

72. Interfacial Shear Stresses A long Fiber. Axis $\left(r_{2} / r_{1}=0.25\right.$, Matrix Yield Strain $=2.0 \%$, Axial Strain fmposed on One Boundary)

73. Matrix Axial Stresses Along Fiber Axis $\left(r_{2} / r_{1}=0.25\right.$, Matrix Yield Strain $=2.0 \%$, Axial Strain Imposed on One Boundary)

74. Radial Variation of Matrix Axial Stresses Near Fiber End $\left(r_{2} / r_{1}=0.25\right.$, Matrix Yield Strain $=2.0 \%$, Axial Strain

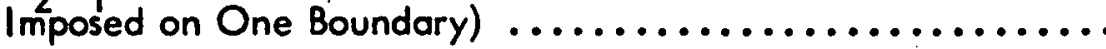

75. Yielding of Matrix at Different Composite Strains $\left(r_{2} / r_{1}=0.25\right.$, Matrix Yield Strain $=2.0 \%$, Axial Strain Imposed on One Boundary)

76. Stress-Strain Curves for Matrix and Composites with Different Fiber Volume Fractions

77. Stress-Strain Curves for Matrices and Corresponding Composites .

78. Fiber Axial Stresses Along Fiber Axis $\left(r_{2} / r_{1}=0.67\right.$, Matrix Yield Strain $=2.0 \%$, Axial Strain Imposed on Entire Outer Boundary)

79. Interfacial Shear Stresses Along Fiber Axis $\left(r_{2} / r_{1}=0.67\right.$, Matrix Yield Strain $=2.0 \%$, Axial Strain Tmposed on Entire Outer Boundary)

80. Matrix Axial Stresses Along Fiber Axis $\left(r_{2} / r_{1}=0.67\right.$, Matrix Yield Strain $=2.0 \%$, Axial Strain Imposed on Entire Outer Boundary)

8i. Radial Variation of Matrix Axial Stresses Near Fiber End $\left(r_{2} / r_{1}=0.67\right.$, Matrix Yield Strain $=2.0 \%$, Axial Strain Imposed on Entire Outer Boundary)

82. Yielding of Matrix at Different Composite Strains $\left(r_{2} / r_{1}=0.67\right.$, Matrix Yield Strain $=2.0 \%$, Axial Strain Imposed on Entire Outer Boundary)

83. Stress-Strain Curves for Matrix and Composite with Axial Strain Imposed on Entire Outer Boundary $\left(r_{2} / r_{1}=0.67\right) \ldots \ldots \ldots$ 
84. Fiber Axial Stresses Along Fiber Axis $\left(r_{2} / r_{1}=0.25\right.$, Matrix Yield Strain $=2.0 \%$, Axial Strain Imposed on Entire Outer Boundary)

85. Interfacial Shear Stresses Along Fiber Axis $\left(r_{2} / r_{1}=0.25\right.$, Matrix Yield Strain $=2.0 \%$, Axial Strain Tmposed on Entire Outer Boundary)

86. Matrix Axial Stresses Along Fiber Axis $\left(r_{2} / r_{1}=0.25\right.$, Matrix Yield Strain $=2.0 \%$, Axial Strain Imposed on Entire Outer Boundary)

87. Radial Variation of Matrix Axial Stresses Near Fiber End $\left(r_{2} / r_{1}=0.25\right.$, Matrix Yield Strain $=2.0 \%$, Axial Strain

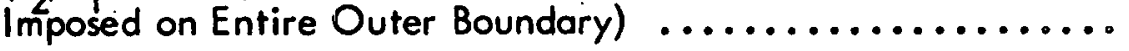

88. Yielding of Matrix at Different Composite Strains $\left(r_{2} / r_{1}=0.25\right.$, Matrix Yield Strain $=2.0 \%$, Axial Strain Imposed on Entire Outer Boundary) 


\section{INTRODUCTION}

Many of the new materials being developed are multiphase or in the composites family. For example, glass fibers are used extensively to reinforce organic matrices. Dispersion strengthened alloys consist of a finely divided second phase distributed in a crystalline matrix. Glass-cermaics make use of controlled crystallization from a glassy melt. Rubber reinforcements are used in glassy polymers to enhance the impact strength of the brittle matrix. In all the cases, the properties of the composite material depend on the properties of the individual components, their distribution, and their physical and chemical interaction.

For the efficient use of composite materials, it is necessary to understand how these materials behave under applied loads. A micromechanics analysis of composite materials is needed to accurately predict the internal stress distributions which are mainly responsible for the ultimate behavior of the composites under given loading conditions. A knowledge of stress distributions helps in the understanding of the material behavior in two ways. As will be discussed in the later sections, the boundary stresses and the accompanying displacements can be used to calculate the modulus of elasticity and Poisson's ratio of the composite materials. These elastic properties predict the macroscopic response of the composite material to the applied loads. Secondly, the internal stresses determine the areas of high stress concentrations in the material. These areas are critical because failure is most likely to start there. The onset of failure may be the formation of a crack in the ceramic matrix composites, or the start of yielding in case of the metal matrices or the initiation of internal crazing in the rubber modified polymers. A number of analytical studies of stress distributions have been performed by the use of simplified physical models. Most of these studies have used elasticity theories to obtain the 
solutions $(1-5)$ : Some of the solutions have been summarized by Holister and Thomas ${ }^{(6)}$. Elasticity solutions of some of the problems are obta ined from Muskhelishvili's ${ }^{(7)}$ solutions using complex variables. Thus solutions exist for simplified models which do not necessarily represent an actual composite material. It is difficult to use the elasticity approoch for solving problems involving complex geometries. In any realistic model of a composite material such complex geometries are unavoidable. The finite element method which has been used in the present investigations allows any complex geometry to be analyzed with equal ease.

The finite element method is not new. For more than a decade, it has been applied extensively to the analysis of large complex structures. Conventional engineering structures can be visualized as an assemblage of structural elements interconnected at a discrete number of nodal points. If the force-displacement relationship for the individual elements is known it is possible, by using various techniques of structural analysis, to derive the properties and study the behavior of the assembled structure.

In an elastic continuum the true number of interconnection points is finite, and here lies the biggest difficulty of the numerical solutions. The difficulty can be overcome (and the approximation made) in the following manner:

(a) The continuum is separated by imaginary lines or surfaces into a number of "finite elements".

(b) The elements are assumed to be iffterconnected at a discrete number of nodal points situated on their boundaries. The displacements of these nodal points will be the basic unknown parameters of the problem, just as in the simple structural analysis.

\footnotetext{
* For all numbered references, see bibliography
} 
(c) A function (or functions) is chosen to define uniquely the state of displacement within each "finite element" in terms of its nodal displacements.

(d) The displacement functions now define uniquely the state of strain within an element in terms of the nodal displacements. These strains, together with any initial strains and the elastic properties of the material will define the state of stress throughout the element and, hence, also on its boundaries.

(e) A system of forces concentrated at the nodes and equilibriating the boundary stresses and any distributed loads is determined.

Once this stage has been reached the solution procedure can follow the standard structural routine. The details such as the formulation of finite element characteristics, mathematical basis, the applicability of the method, etc. can be found in the text written by Zienkiewicz ${ }^{(8)}$. In the present investigations, existing computer programs were used with minor changes. The details and reference will be given in appropriate sections of this thesis.

The next two sections deal with the particulate composites. In the first of the two, axisymmetric representation of the composite has been assumed. The effect of the volume fraction of the inclusions on the internal stresses and elastic constants is discussed in detail. The results are compared with the available experimental data and with some of the predictions obtained by using elasticity theory. Another important problem of the particulate composites, namely the effect of an interface, which received very little attention from other investigators has also been studied. In the third section a general three-dimensional model of the composite material has been analyzed. The emphasis, once again, has been on the internal stresses and the elastic constants. Some conclusions have been drawn concerning the use of a complex and relatively expensive computer program to analyze the general three-dimensional solids. 
Sections IV and $V$ deal with the discontinuous fibrous composites. In Section IV, an elastic analysis has been used to study in detail the effect of an interface. It covers a number of areas such as weak and strong interface, effect of interface on internal stresses and elastic constants, and effect of fiber end conditions. In Section V, an elastic-plastic analysis of fibrous composites has been performed to study the internal stresses. Effect of matrix yielding on the composite stress-strain. curve has been investigated. 


\section{AXISYMMETRIC ANALYSIS OF PARTICULATE COMPOSITES}

Introduction

The composites which consist of particles of one phase dispersed in a second phase are usually referred to as the particulate composites. The filler particles in a particulate composite have no long dimension. It may be round, square or even triangular, but the dimensions of its sides are approximately equal. In a particulate composite, the matrix and dispersed particles share the load. Strengthening of particulate-reinforced composite occurs initially when the dispersed particles restrict the matrix deformation by a mechanical restraint. The magnitude of the restraint is unknown and complex, but it is a function of the interparticle spacing and the ratio of the elastic properties of the matrix and particle. A micromechanics analysis is needed to understand the influence of these factors on the behavior of composite materials.

Previous investigators have discussed the stresses oround spherical inclusions as applied to porous ceramics or spherically filled ceramic particulate composites $^{(9-12)}$. The 'Goodier solution ${ }^{(1)}$, which was used for all of these analyses, applies only to a single inclusion or cavity; thus, interactions between particles are ignored when the theory is applied to actual composites in which particle interactions can influence the stress state. Even in particulate composites with only 30 vol\% particles, local regions of the composite may have effective concentrations of 50 to 60 vol\% as a result of non-uniform particle concentrations. The results presented indicate how the interfacial stresses are influenced by particle spacing. 
Approximations and Boundary Conditions

The present investigations were carried out using an analysis of axisymmetric solids. In the finite element approximation of axisymmetric solids, the continuous structure or medium is replaced by a system of axisymmetric elements interconnected at nodal circles. It was assumed that the porous or filled ceramics (assumed to possess symmetry) could be approximated by a unit cell (Fig. 1) which when rotated $360^{\circ}$ around axis $A D$ produces a hemisphere embedded within a cylinder. The interparticle spacing is equal to $2\left(r_{1}-r_{2}\right) ; r_{1}$ and $r_{2}$ are shown in Fig. $?$. The volume percent of filler particles or cavities (radius $=r_{2}$ ) can be altered and calculated from the ratio $r_{2} / r_{1}$ (note $A B=B C=C D=A D$ in Fig. 1). This axisymmetric representation of the composite only approximates its real packing and structure. These axisymmetric cells are not an actual repetitive unit but are related in their dimensions to the interparticle spacing. A 3-dimensional computer program without restrictions (e.g., using tetrahedral elements) has also been used (in Section III) to truly model a particulate composite.

The unit cell shown in Fig. $I$ is subdivided into small elements. The finite element grids used for six different interparticle spacings are shown in Figs. 2-7. The finite element method permits calculation of the stresses in all the elements and the displacements at the nodal circles for any loading and boundary conditions. It is assumed that the composite is strained in the $z$-direction and that no tractions are applied in the r-direction. By symmetry, on the boundary $A B C D($ Fig. 1) the shear stresses are:

$$
\tau_{r z}=\tau_{z r}=0
$$

The sides $A B$ and $B C$ remain parallel to their original positions after they are displaced due to strain in the $z$ direction, whereas the normal displacements of $A D$ and $D C$ are zero. Thus, $A B$ and $B C$ will undergo normal displacements, and the traction in the $r$ direction must be zero so that:

$$
\int_{B C} \sigma_{r} d z=0
$$




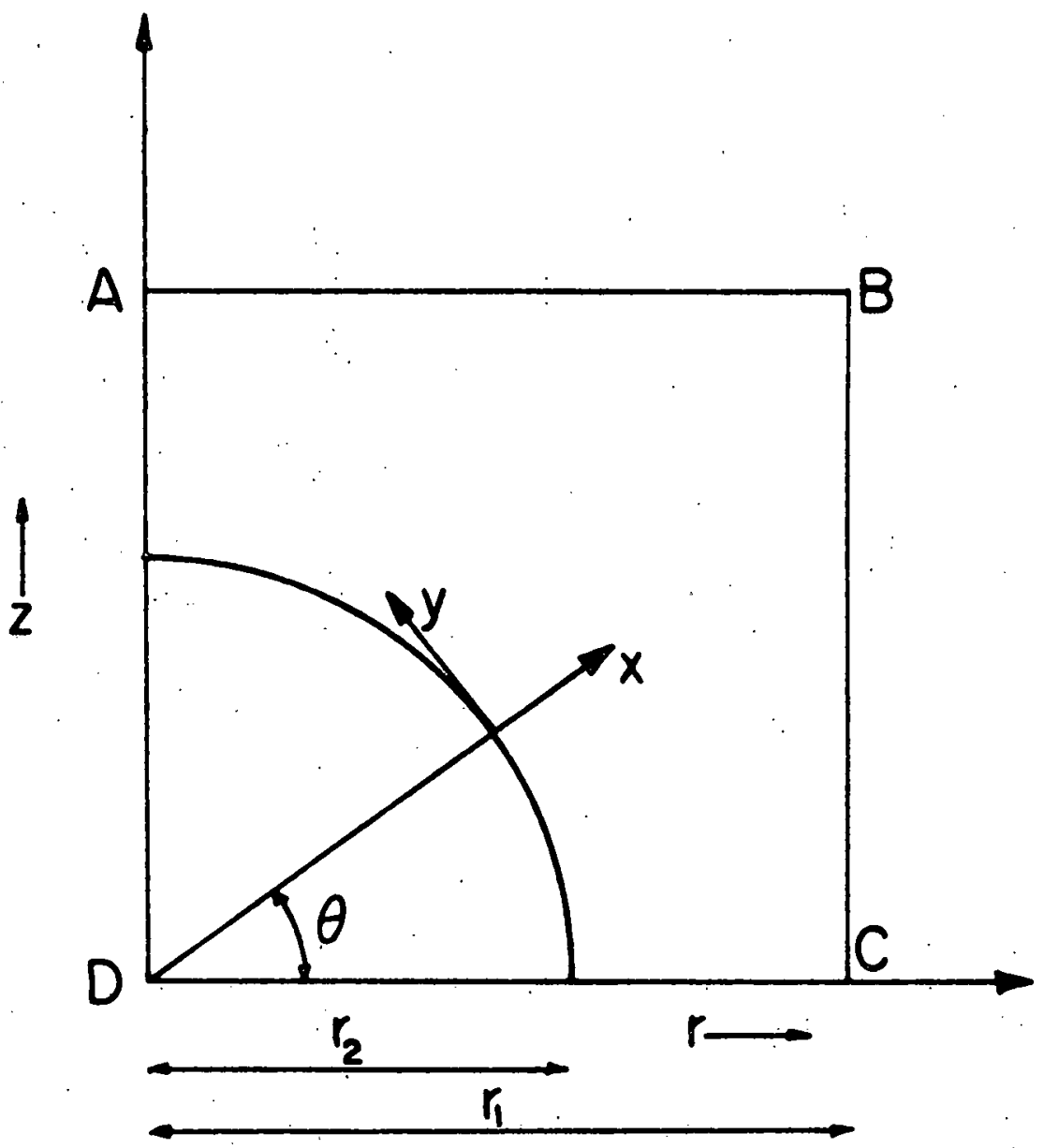

Fig. 1. Unit Cell for Axisymmetric Representation of Sphere-filled Composite 


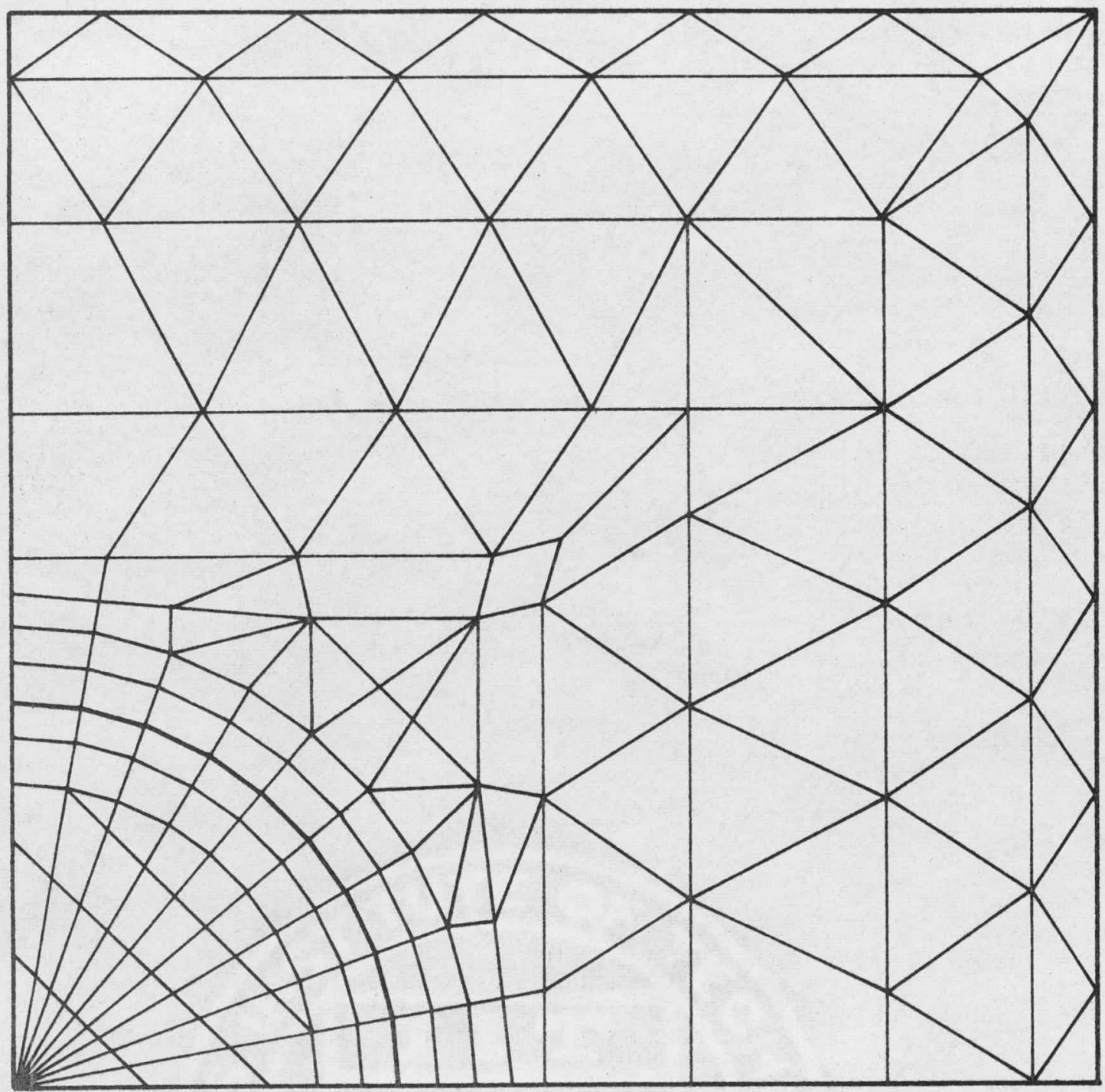

Fig. 2. Finite Element Grid for $r_{2} / r_{1}=0.357$ 
$a$

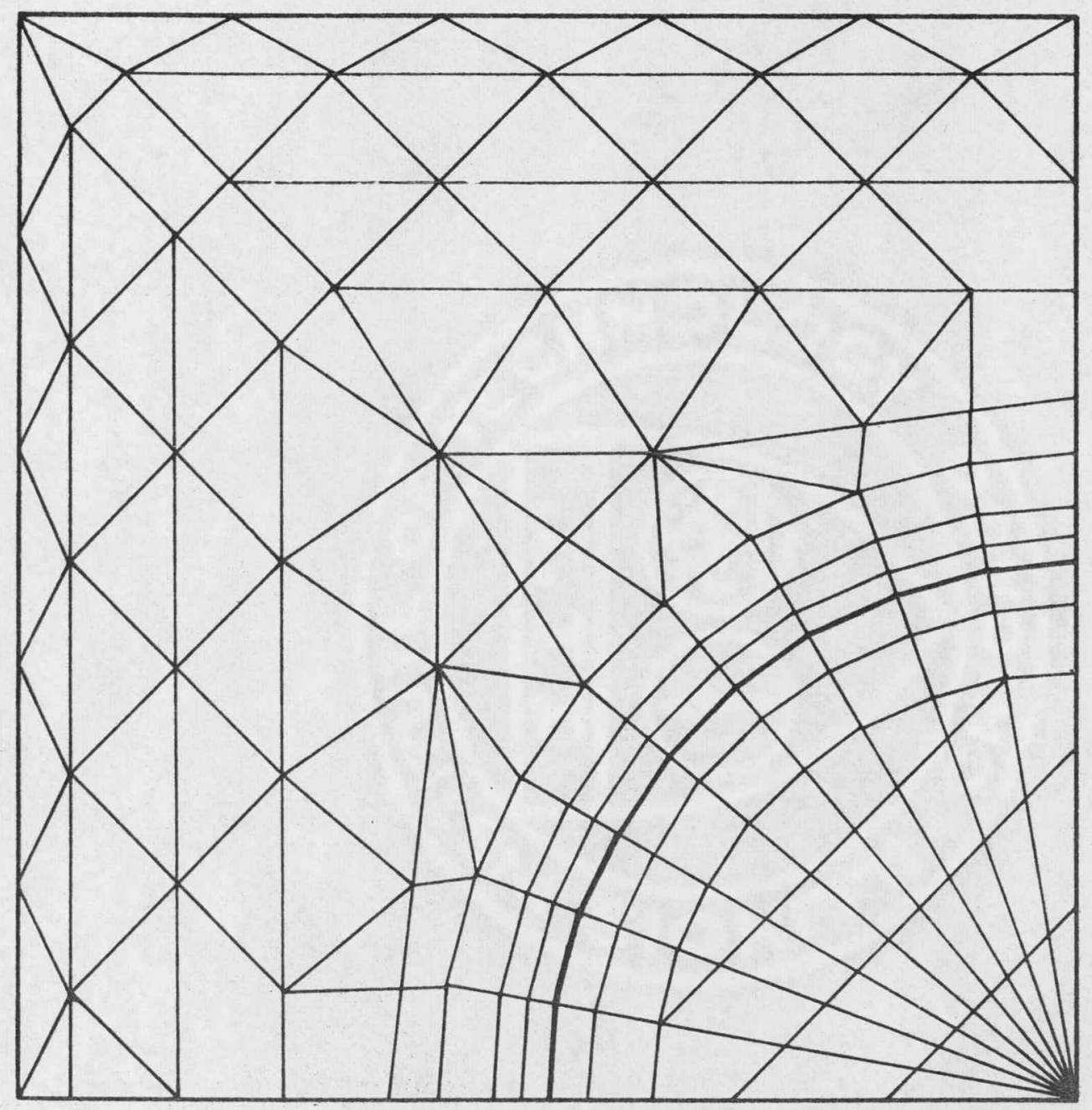

ह 


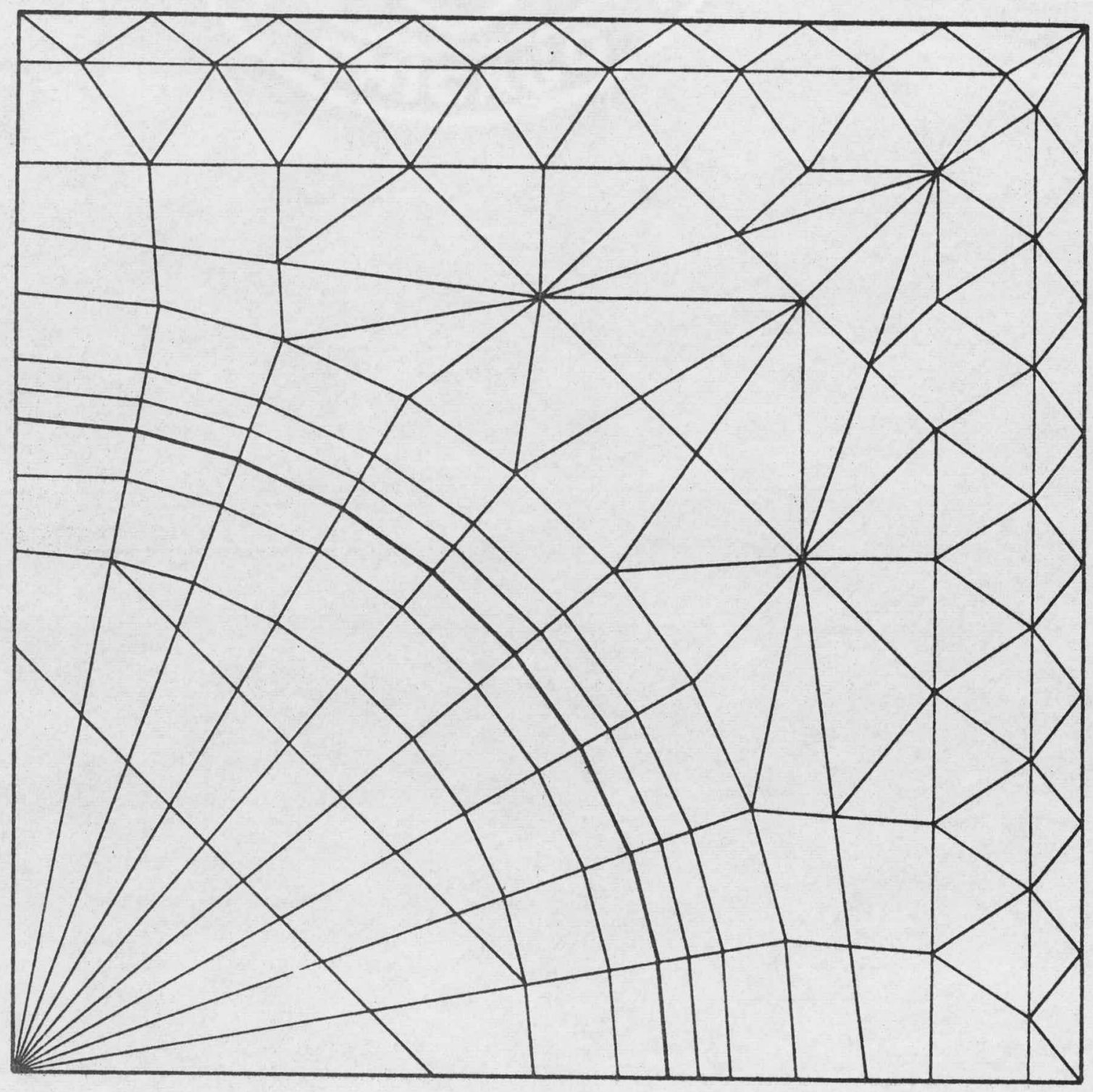

Fig. 4. Finite Element Grid for $r_{2} / r_{1}=0.615$ 


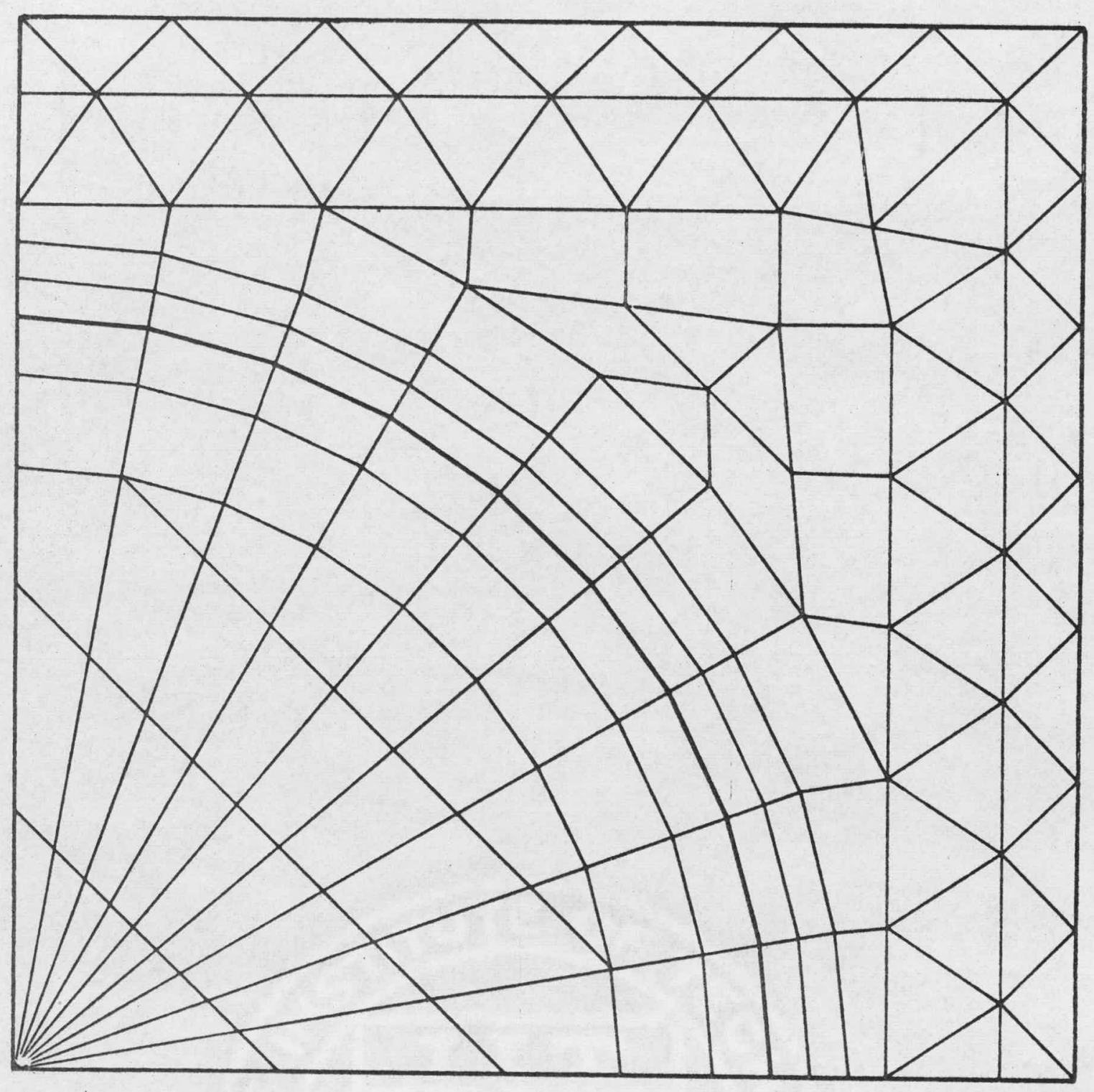

Fig. 5. Finite Element Grid for $r_{2} / r_{1}=0.714$ 


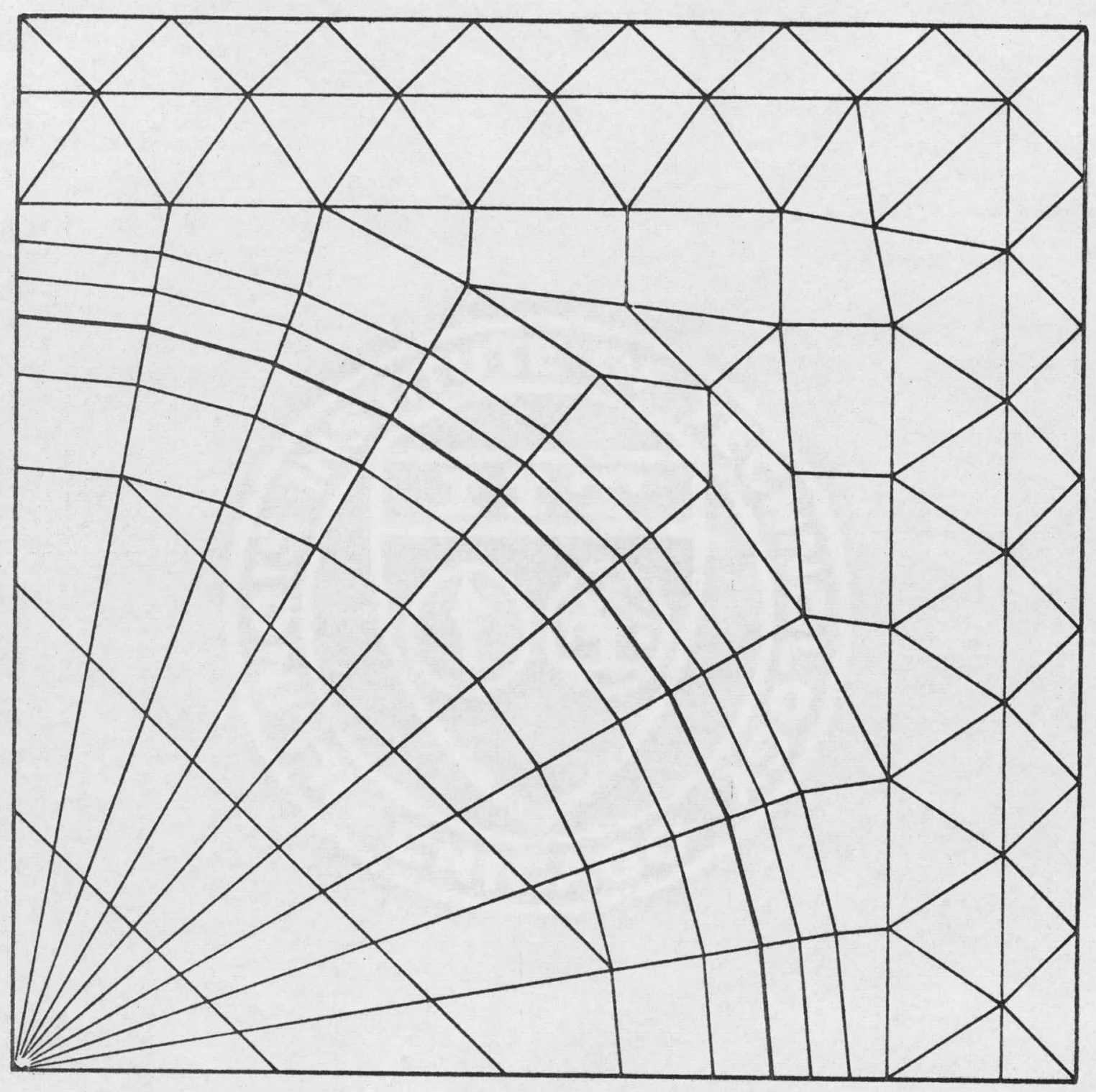

Fig. 5. Finite Element Grid for $r_{2} / r_{1}=0.714$ 


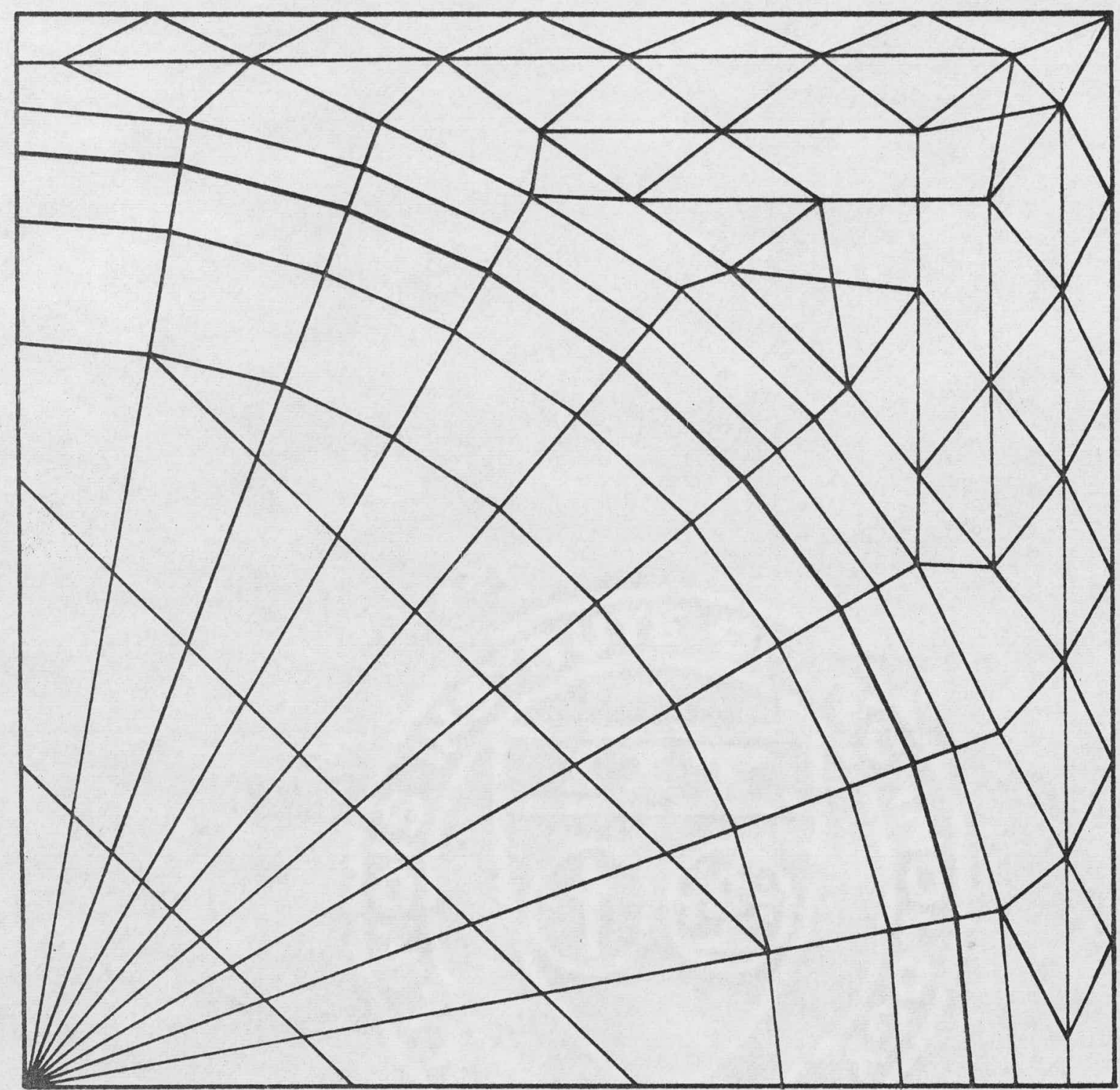

Fig. 7. Finite Element Grid for $r_{2} / r_{1}=0.870$ 
where the integral is replaced by a summation in the finite element method.

The following assumptions were made concerning the material:

1. Filler particles and cavities are spherical and of uniform size; packing of particles can be represented by an axisymmetric element (Fig. 1).

2. Both filler and matrix materials obey elastic stress-strain relationships.

3. Perfect bonding exists between filler and matrix (continuity of displacements at the interface).

- The calculations were made on a large digital computer* by using a computer program for the analysis of axisymmetric solids written by E. L. Wilson (13) . The boundary conditions were prescribed in the mixed mode i.e., the displacements were prescribed on some of the boundaries whereas tractions were prescribed on the others. The prescribed boundary displacements were selected to obtain the desired composite strains. The average composite stress was calculated from a knowledge of the stresses in the elements at the boundary of the unit cell. The composite stresses and strains are used to calculate the composite modulus of elasticity and Poisson's ratio. The details of the procedure to satisfy the boundary conditions and to calculate composite stress, strain, modulus of elasticity and Poisson's ratio have been given in the Appendix.

\section{Internal Stresses}

The internal triaxial stresses were calculated throughout the volume (in each element shown in Figs. 2-7) of porous and filled cermaics with cavity or filler volume contents up to 43.83 percent (minimum interparticle spacing $=0.26 r_{2}$ ' where $r_{2}=$ radius of spherical particles or covities).

For a porous cermic the following properties were assumed for the ceramic with no porosity:

*. Univac 1108, Univac Div., Sperry Rand Corp., Philadelphia, Pa. 


$$
\begin{aligned}
& E \text { (modulus of elasticity) }=10 \times 10^{6} \mathrm{psi} \\
& \nu \text { (Poisson's ratio) }=0.24
\end{aligned}
$$

Two filled ceramic systems were analyzed. The component properties assumed for system 1 were:

$$
\text { Glass matrix } \begin{aligned}
E & =11.8 \times 10^{6} \mathrm{psi} \\
\nu & =0.197 \\
\text { Alumina filler } E & =60.4 \times 10^{6} \mathrm{psi} \\
\nu & =0.257
\end{aligned}
$$

These properties are those reported by Hasselman and Fulrath ${ }^{(14)}$.

For the system 2, the values were

$$
\begin{aligned}
\text { Glass matrix } E & =11.8 \times 10^{6} \mathrm{psi} \\
\nu & =0.197 \\
\text { Tungsten filler } E & =52.2 \times 10^{6} \mathrm{psi} \\
\nu & =0.1985
\end{aligned}
$$

as reported by Hasselman and Fulrath ${ }^{(15)}$.

Volume contents of void or filler can be calculated from the ratio $r_{2} / r_{1}$, by assuming an appropriate packing of the cylindrical region analyzed. Three different arrangements, namely sphere in a cylinder, hexagonal packing of cylinders and square packing of cylinders (this coincides with the cubic array of spheres), have been shown in Fig. 8. Expressions for the volume fractions have also been indicated on the figure. The ratios $r_{2} / r_{1}$ and filler or void contents corresponding to the above packings are given in Table 1 . The volume fractions used in this chapter were calculated by assuming a sphere in a cylinder. Significance of assuming a different packing will be discussed in the next chapter. 

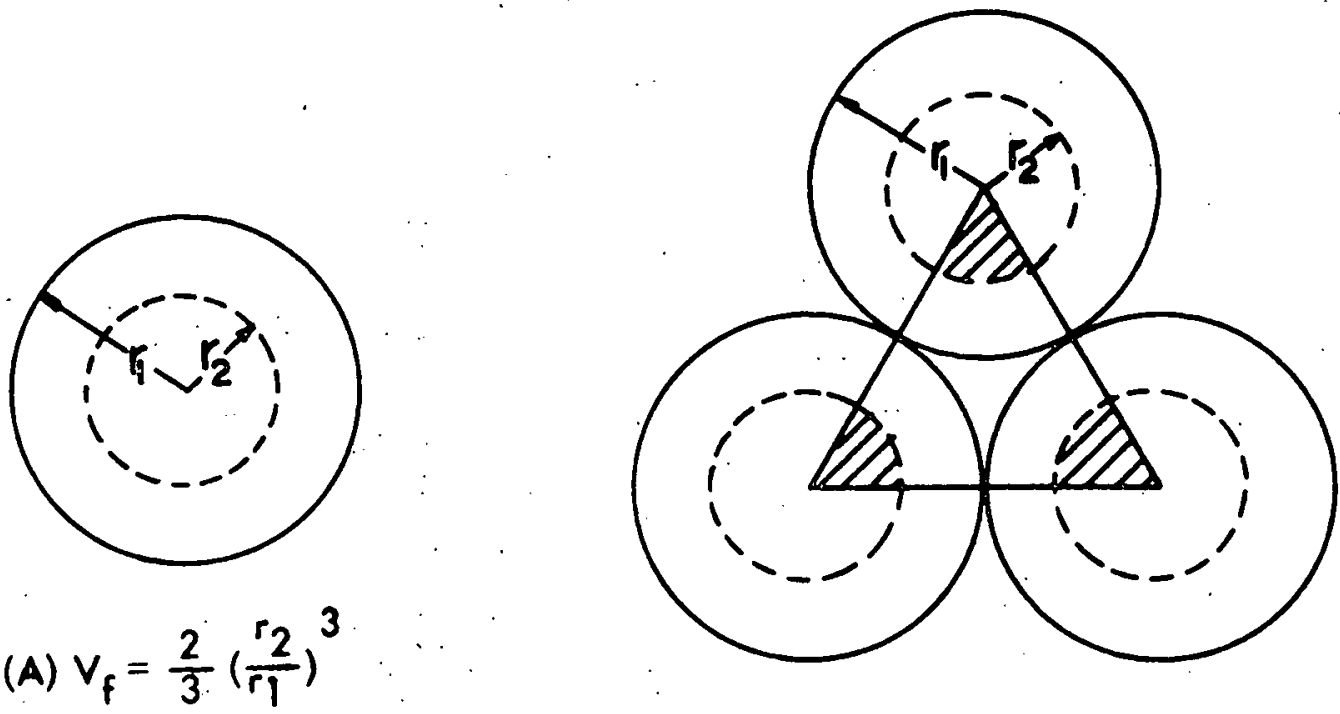

(B) $V_{f}=\frac{\pi}{36 \times 0 . n T}\left(\frac{r_{2}}{r_{1}}\right)^{3}$

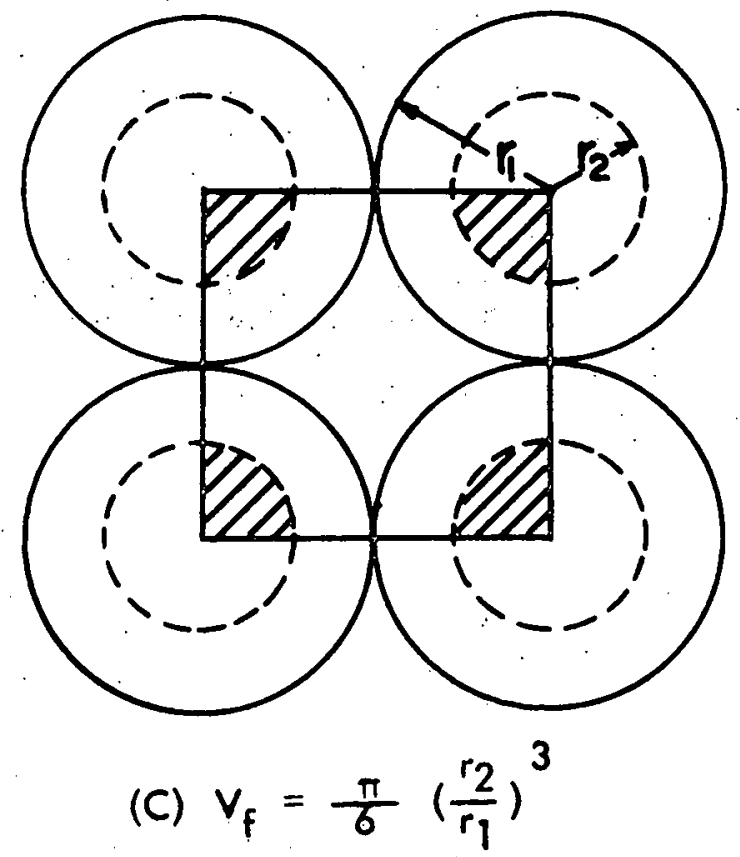

Fig. 8. Volume Packing (A) Sphere in a Cylinder, (B) Hexagonal Array, and (C) Square or Cubic Array 
Table 1. The Relation Between Particle Spacing and Volume Fraction

\begin{tabular}{cccc}
\hline${ }^{2} / r_{1}$ & $\begin{array}{c}\text { Sphere in a } \\
\text { Cylinder }\end{array}$ & $\begin{array}{c}\text { Hexagonal } \\
\text { Array }\end{array}$ & $\begin{array}{c}\text { Square or } \\
\text { Cubic Array }\end{array}$ \\
\hline 0.357 & 3.04 & 2.75 & 2.38 \\
0.500 & 8.33 & 7.56 & 6.54 \\
0.615 & 15.54 & 14.09 & 12.20 \\
0.714 & 24.30 & 22.03 & 19.08 \\
0.833 & 38.58 & 34.99 & 30.30 \\
0.870 & 43.83 & 39.75 & 34.43 \\
0.952 & 57.59 & 52.23 & 45.23 \\
\hline
\end{tabular}

The stresses around the spherical cavity are shown in Figs. 9 and 10. The stresses are represented as a ratio $\sigma / \bar{\sigma}_{z}$, where $\bar{\sigma}_{z}$ is the average stress applied to the composite. Thus, the ratio represents the stress concentration around the cavity. The stress system is defined in Fig. 9. Fig. 2 shows that there are 9 finite elements around the interface of the cavity; the calculated stresses are assumed to. act at the center of each element: In all of the cases shown, the radial and tangential stresses at the interface are almost equivalent to the principal stresses and thus the shear stresses $\left(e . g_{.}, \tau_{x y}\right)$ are nearly zero. The absolute value of maximum shear stress is calculated from:

$$
\tau_{\text {max }}=\left|\frac{\sigma_{1}-\sigma_{2}}{2}\right|
$$

The variation of stresses at the pole $\left(\theta=90^{\circ}\right)$ and equator $\left(\theta=0^{\circ}\right)$ of a spherical cavity are shown in Fig. 11 as a function of porosity percent. The curves for stresses were extrapolated to $V_{f}=0$ percent. For tangential stresses at the pole, perfect agreement is obtained with the Goodier solution for an inclusion in an infinite matrix. For the stress at the equator, the agreement is within five percent of the theoretical solution of Goodier. The difference results from the size of the 


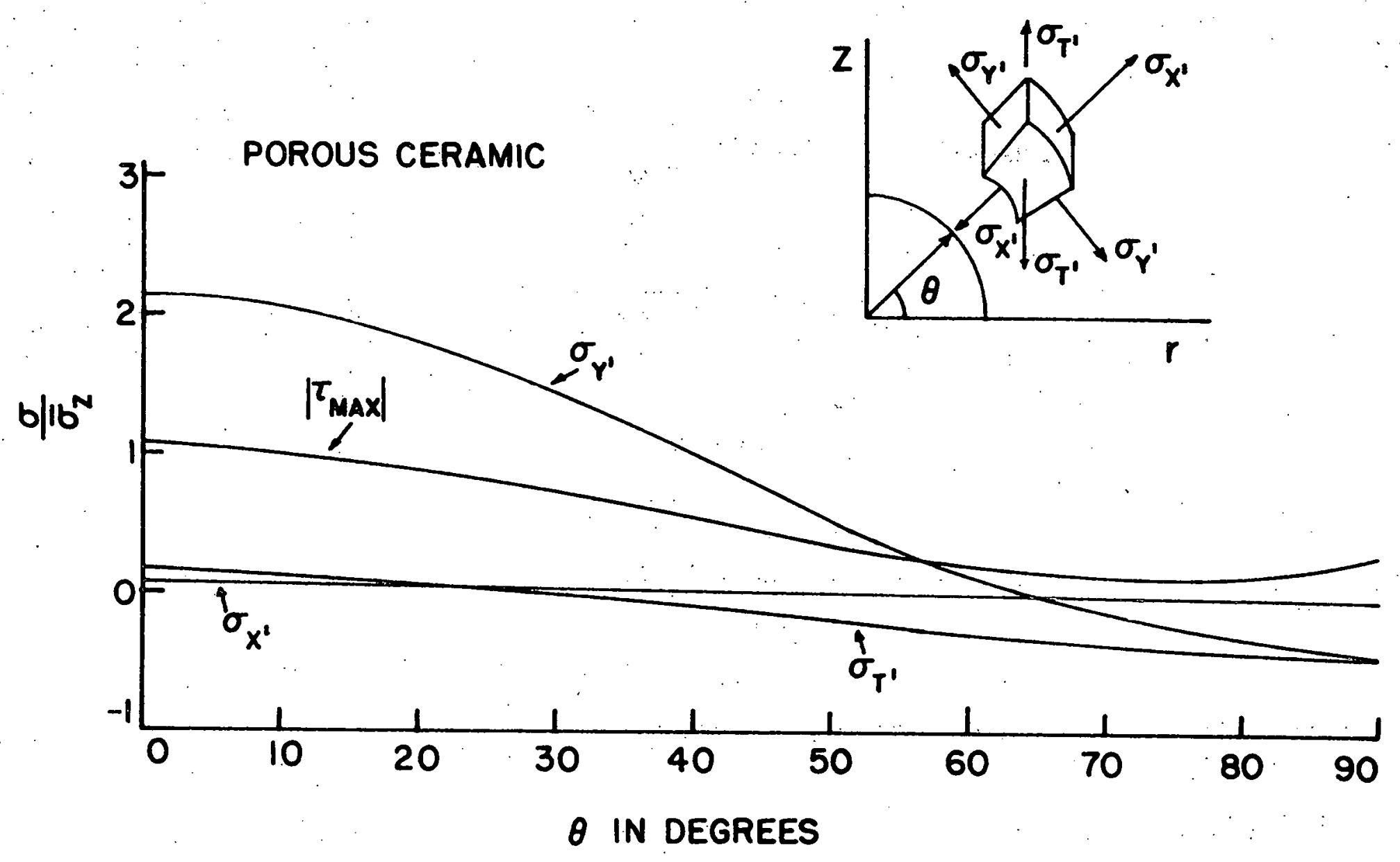

Fig. 9. Matrix Stresses at Pore Boundary in Porous Ceramic with $\frac{r_{2}}{r_{1}}=0.615$ 


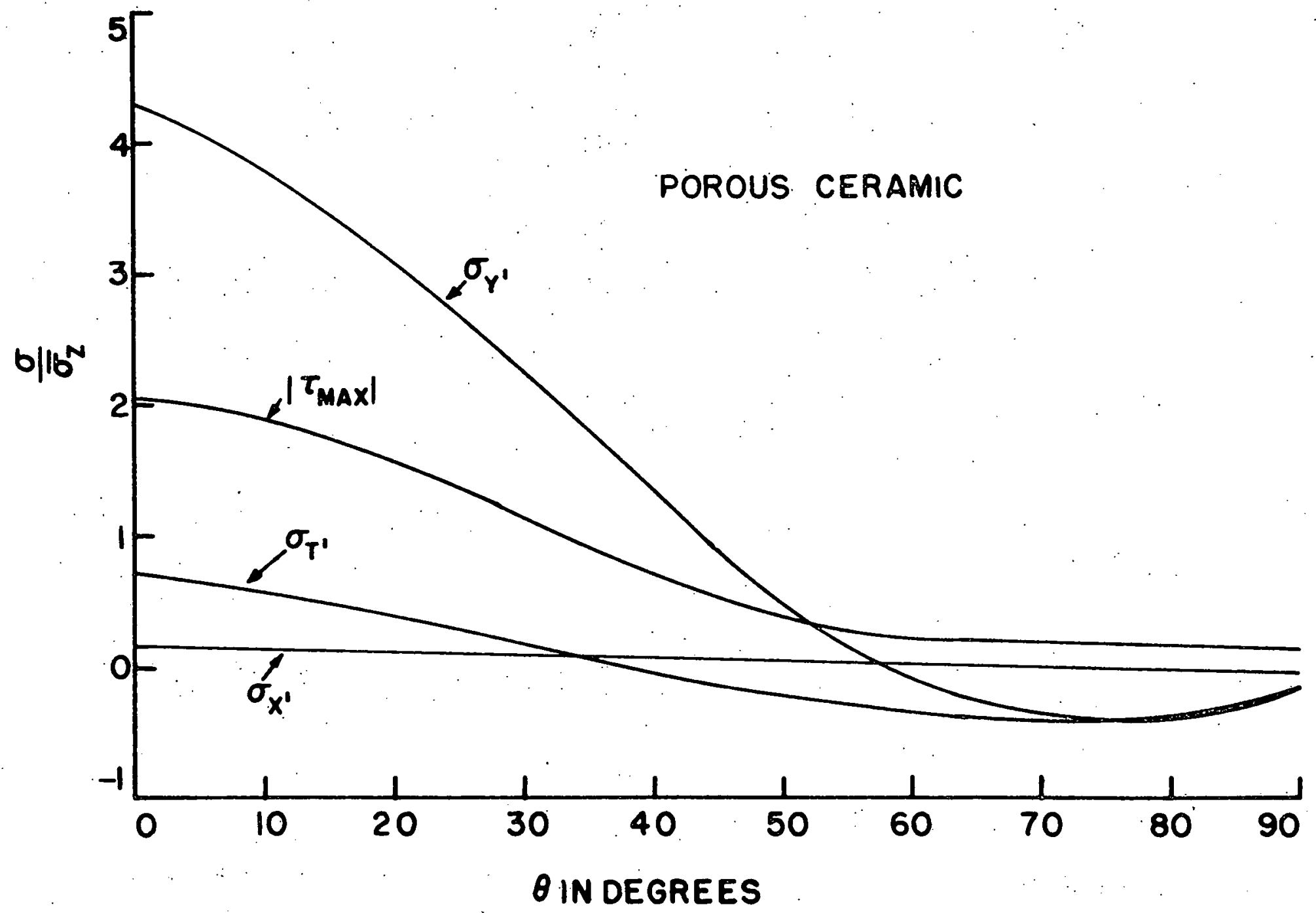

Fig. 10. Matrix Stresses at Pore Boundary in Porous Ceramic with $r_{2} / r_{1}=0.870$ 


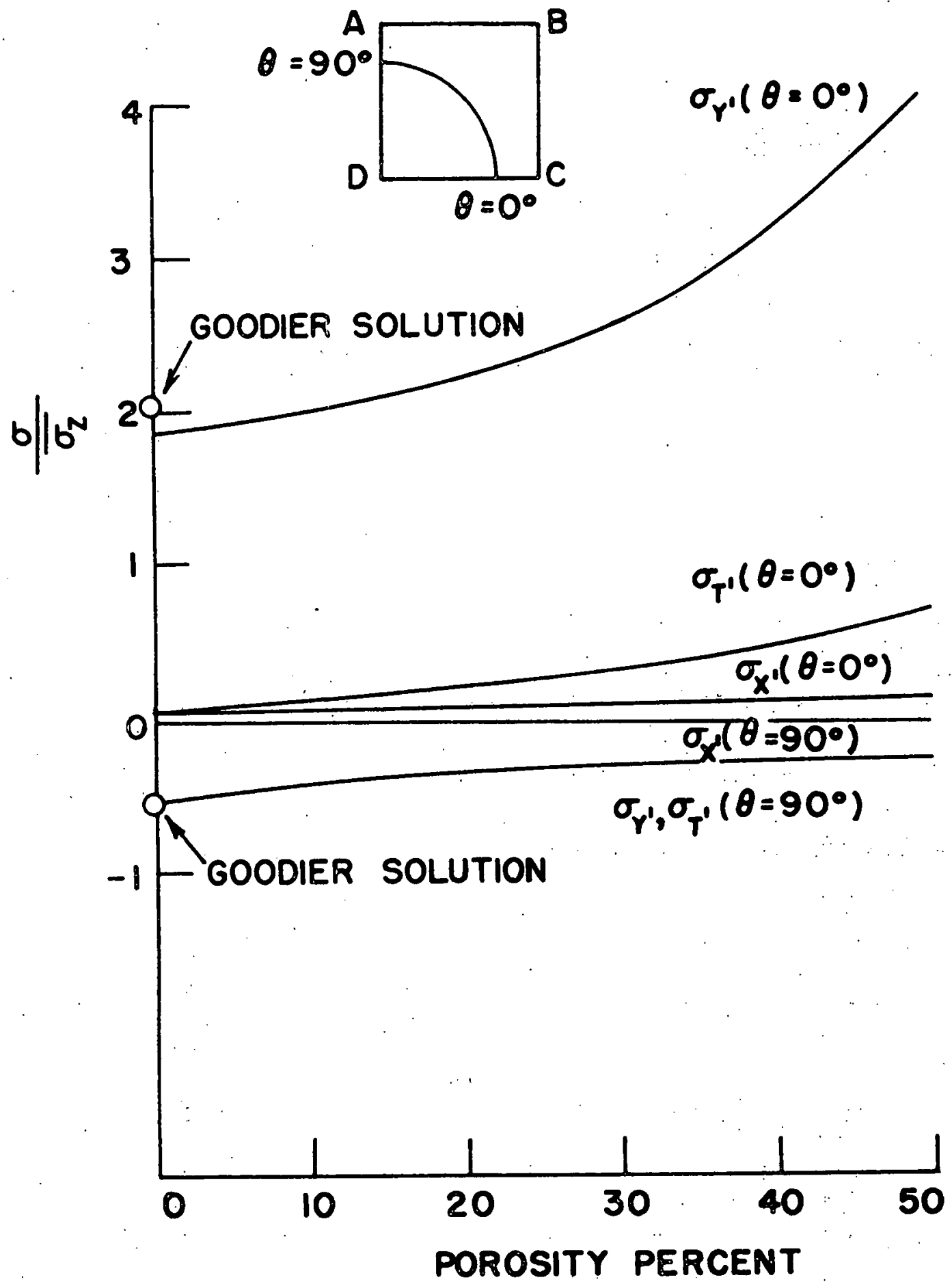

Fig. 11. Pore Boundary Stresses as a Function of Porosity (vol.\%) 
finite element and the averaging which thus occurs. Also, the stress normal to the cavity boundary $\left(\sigma_{x}\right)$ should be zero at the boundary. The very small positive or negative value obtained in the present solution results again from the size taken for the finite element.

The decay of stresses away from the interface is shown in Fig. 12. The stresses have been plotted beginning from the interface and continuing along the boundaries $A D$ and $C D$ as shown. Only the stress normal to the boundary has been plotted. It can be seen that for a low volume percent filler (3\%) the normal stress along CD reduces to the average stress, $\bar{\sigma}_{\mathbf{Z}^{\prime}}$ at the midpoint between two cavities while the normal stress along $A D$ approaches zero as it should. However, the results for higher volume percent show that the stress concentrations persist even at the midpoint between two cavities because of the close cavity spacing.

The stresses around the interface between the $\mathrm{Al}_{2} \mathrm{O}_{3}$ particles and glass matrix are shown in Figs. 13 and 14. The stress system is the same as that defined in Fig. 9. The variation of stresses at the pole $\left(\theta=90^{\circ}\right)$ and the equator $\left(\theta=0^{\circ}\right)$ of an $\mathrm{Al}_{2} \mathrm{O}_{3}$ particle (assumed spherical) is shown in Fig. 15, again the theoretical solutions of Goodier agree quite well at zero volume percent filler.

The variation of stresses was not reported for the glass $-W$ system. However, these stresses are very similar to those for the glass- $\mathrm{Al}_{2} \mathrm{O}_{3}$ system. These analyses show that, for porous composites, the stresses around the pores are influenced by interactions from neighboring pores when $r_{2} / r_{1}=0.5$ or when the spacing between pores is equal to one pore diameter. For the filled composites, interactions are not important until the inclusions are within approximately $1 / 4$ diameter of one another. Broutman and Panizza ${ }^{(16)}$ made some micromechanics studies with rubber reinforced glassy polymer. This represents an interesting case where the reinforcing particles have a modulus of elasticity much less than that of the glassy polymer 


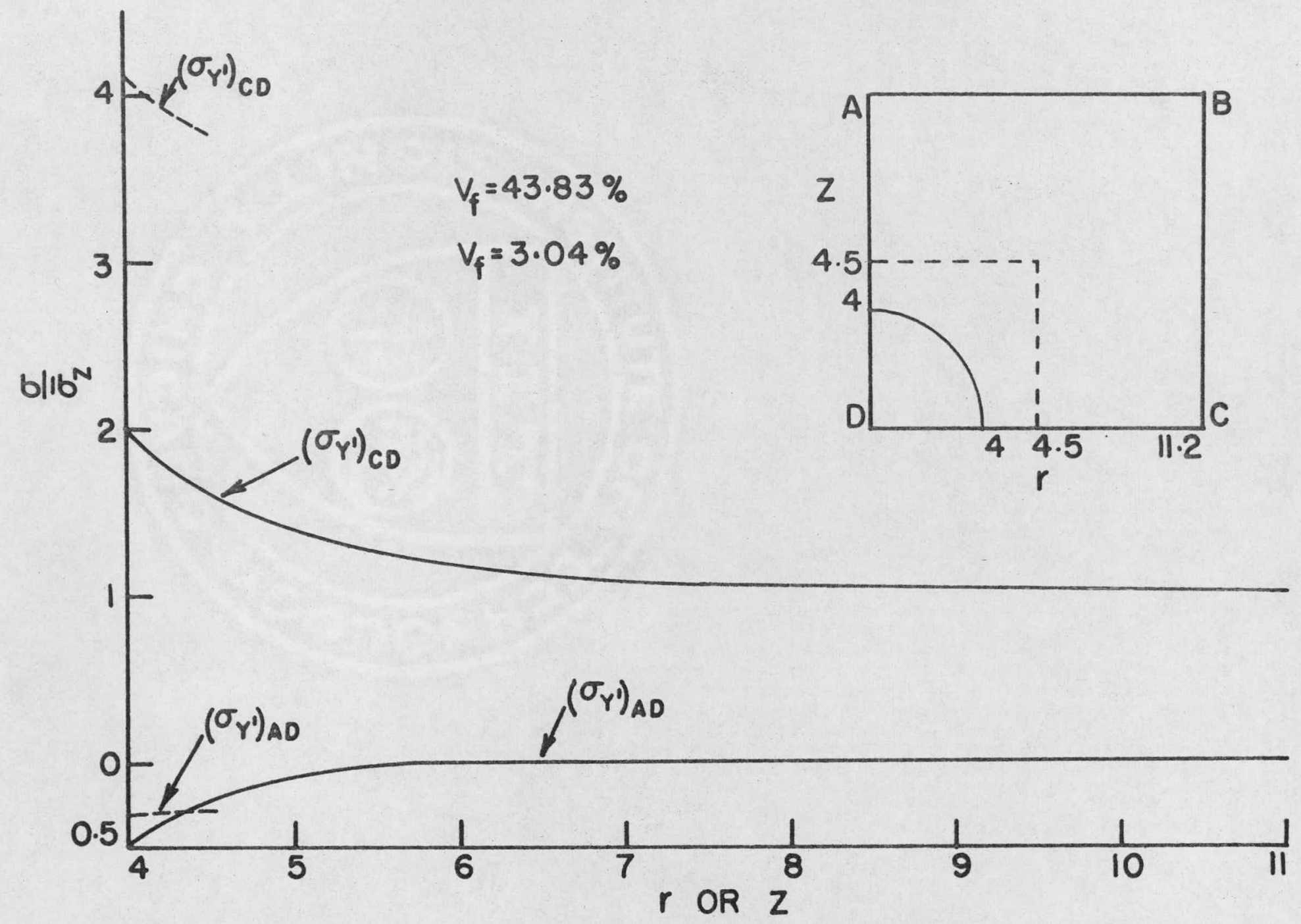

Fig. 12. Matrix Stresses in the Composite 


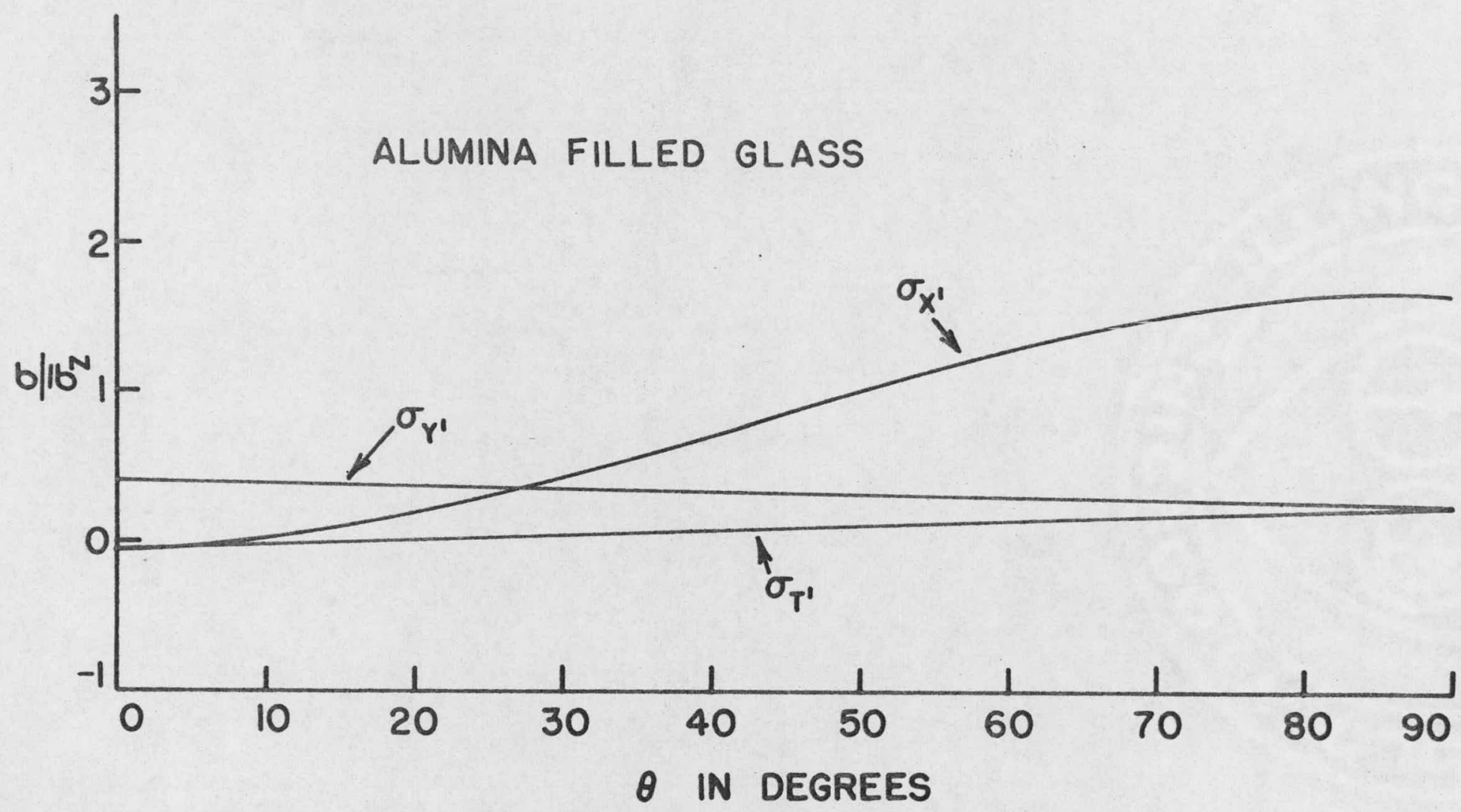

Fig. 13. Matrix Stresses at Interface in Alumina-filled Glass with $r_{2} / r_{1}=0.357$ 


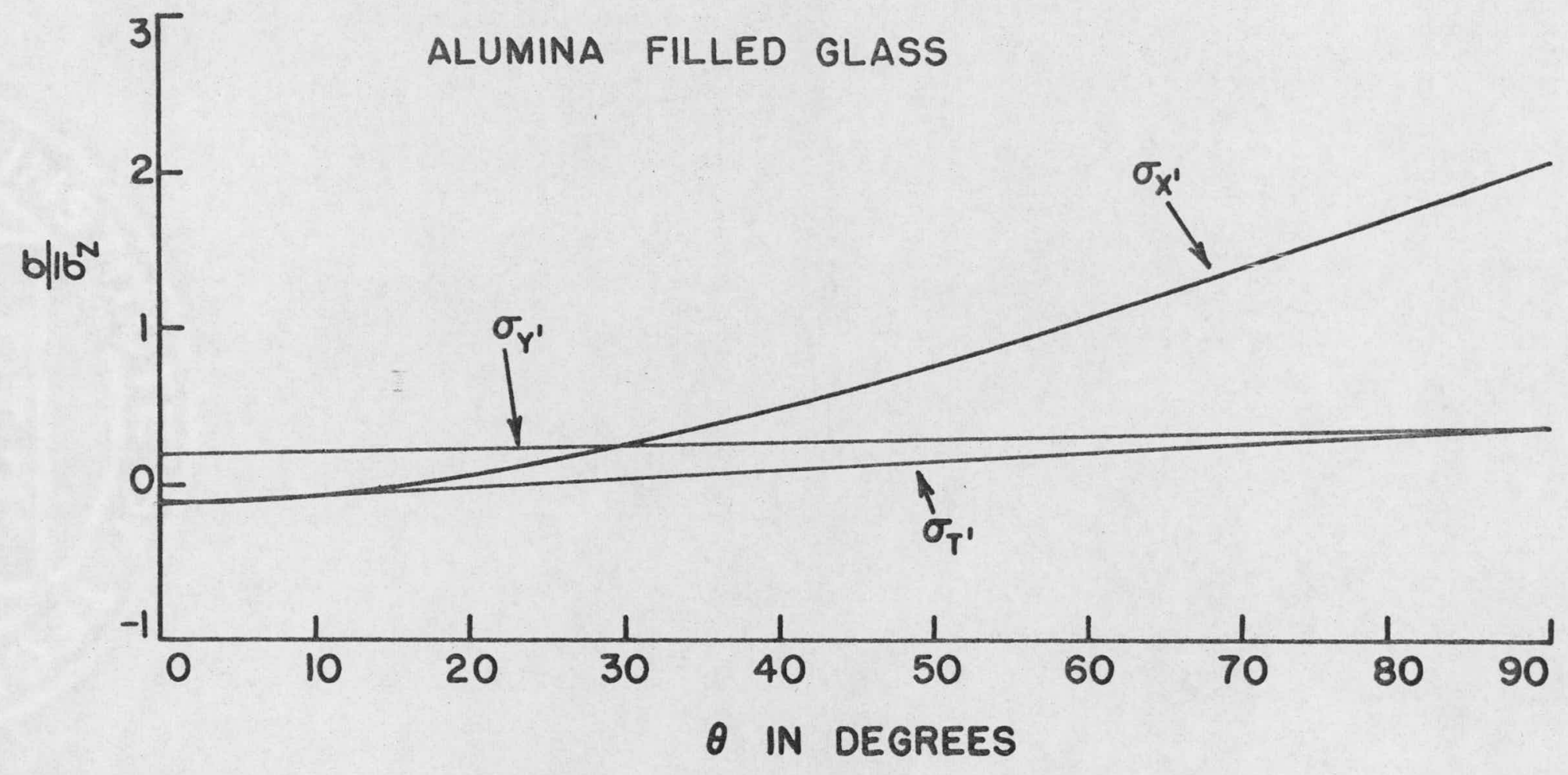

Fig. 14. Matrix Stresses at Inferface in Alumina-filled Glass with $r_{2} / r_{1}=0.870$ 


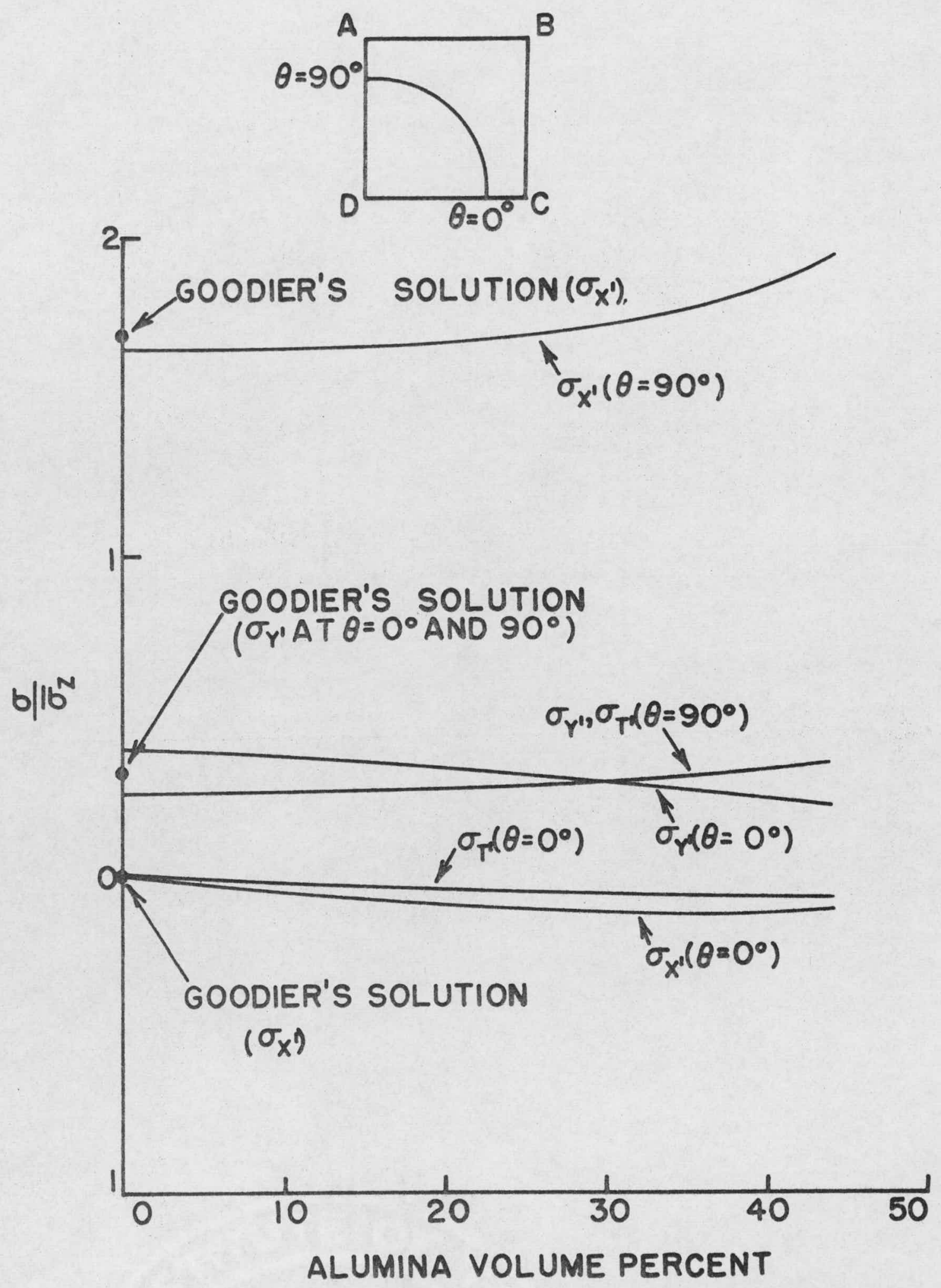

Fig. 15. Interfacial Stresses in Alumina filled Glass as a Function of Volume Fraction of Alumina 
matrix and Poisson's ratio is greater than that of the matrix. The results of the present study qualitatively confirm those of Broutman and Panizza.

\section{Elastic Constants}

The predicted moduli of elasticity and Poisson's ratios are shown in Figs. 16, 17 and 18 and Table 2. The modulus and Poisson's ratio decrease with increasing porosity. Experimental data of Fryxell and Chandler $(17)$ agree quite well with the predicted values; the data of Manning et al. ${ }^{(18)}$ do not agree so well. It can be seen that the normalized experimental data of Fryxell and Chandler do not agree well with those of Manning et al. This may be due to the inherent differences in the material used and also partially to the experimental techniques used by the investigators. Therefore, one simple theory may not be expected to predict the experimental data concerning different materials with equal accuracies.

The modulus of elasticity of a composite increases as the filler content increases (Figs. 17 and 18). The predicted values are compared in Figs. 17 and 18 with the experimental results of Hasse Iman and Fulrath ${ }^{(14,15)}$, the excellent agreement between the experimental and predicted values is shown.

In the present analysis, the moduli of elasticity were calculated on the basis of the actual stress distribution in the composites when the continuous medium is approximated by a system of discrete elements. Therefore, the present studies permit a unique calculation of elastic moduli and do not present the bounded solutions (such as that of Hashin and Shtrikman ${ }^{(3)}$ ) previously used to predict experimental results. However, Hashin and Shtrikman's lower bound agrees well with the predictions of the present analysis and with the experimental results of Hasselman and Fulrath $(14,15)$. Moduli of elasticity calculated from the lower bound of Hashin and Shtrikman's the ory are also shown in Table 2. 


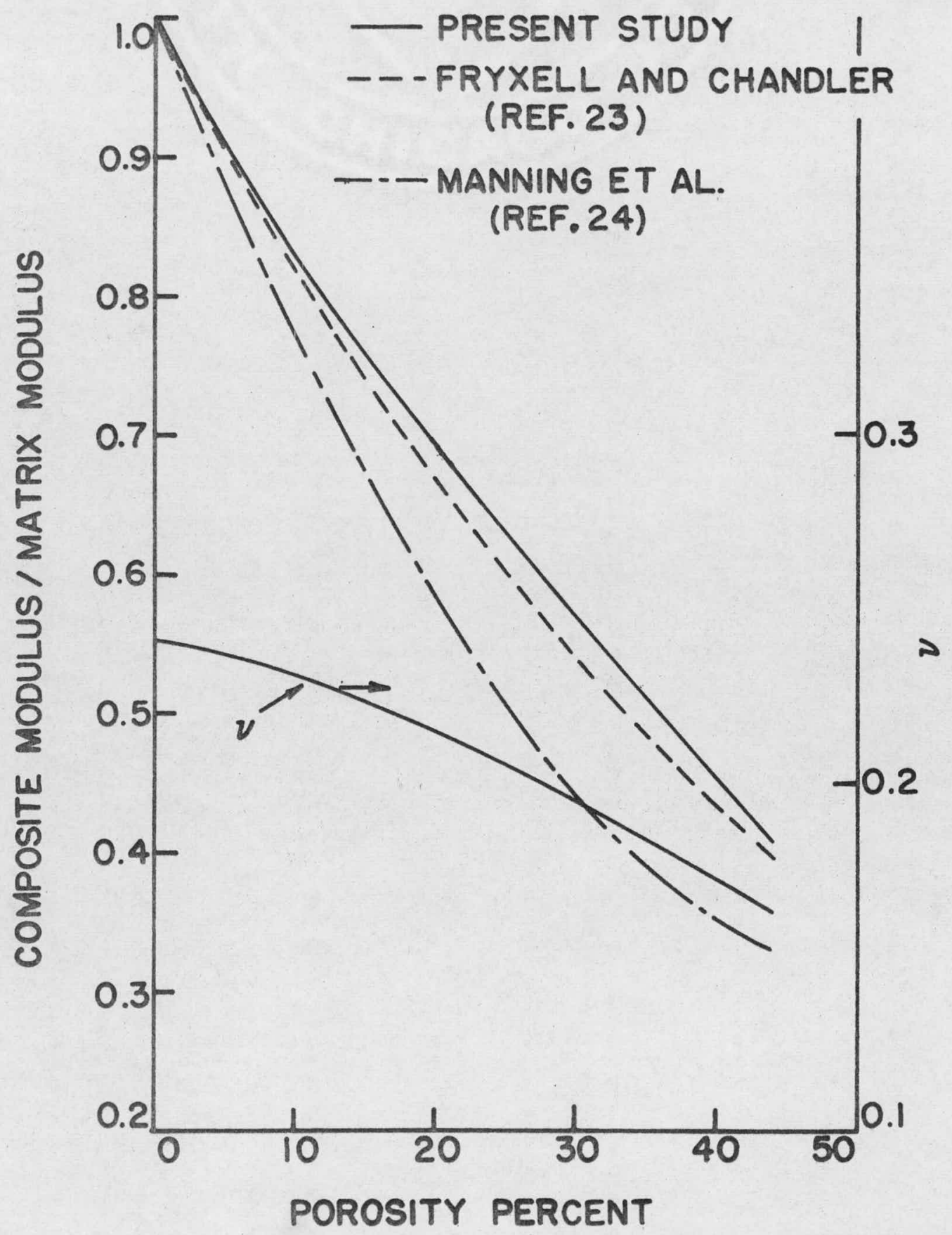

Fig. 16. Comparison of Predicted Modulus of Elasticity of Porous Ceramic with Experimental Data and Prediction of Poisson's Ratio 


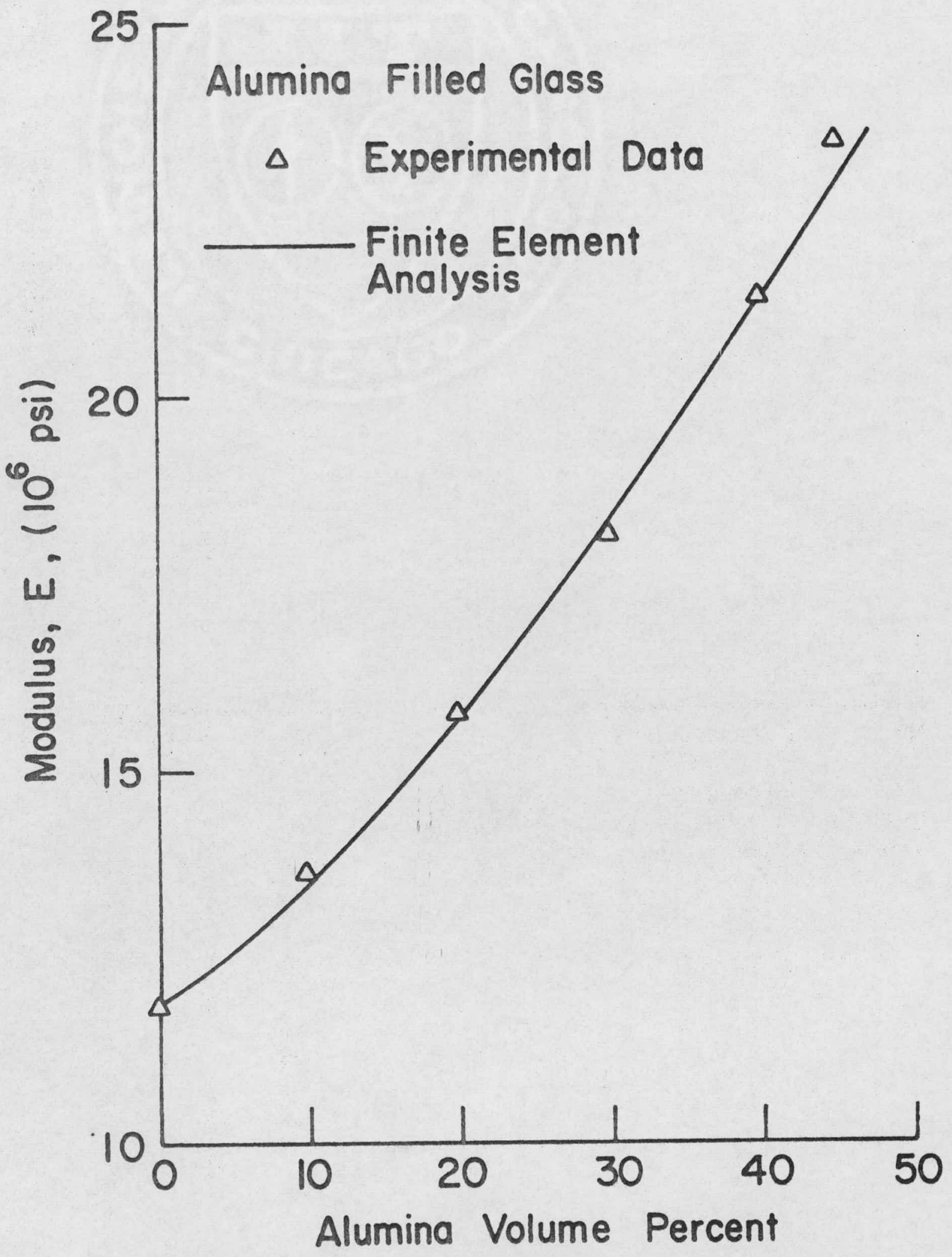

Fig. 17. Comparison of Predicted Modulus of Elasticity of AluminaFilled Glass with Experimental Data of Reference 14. 


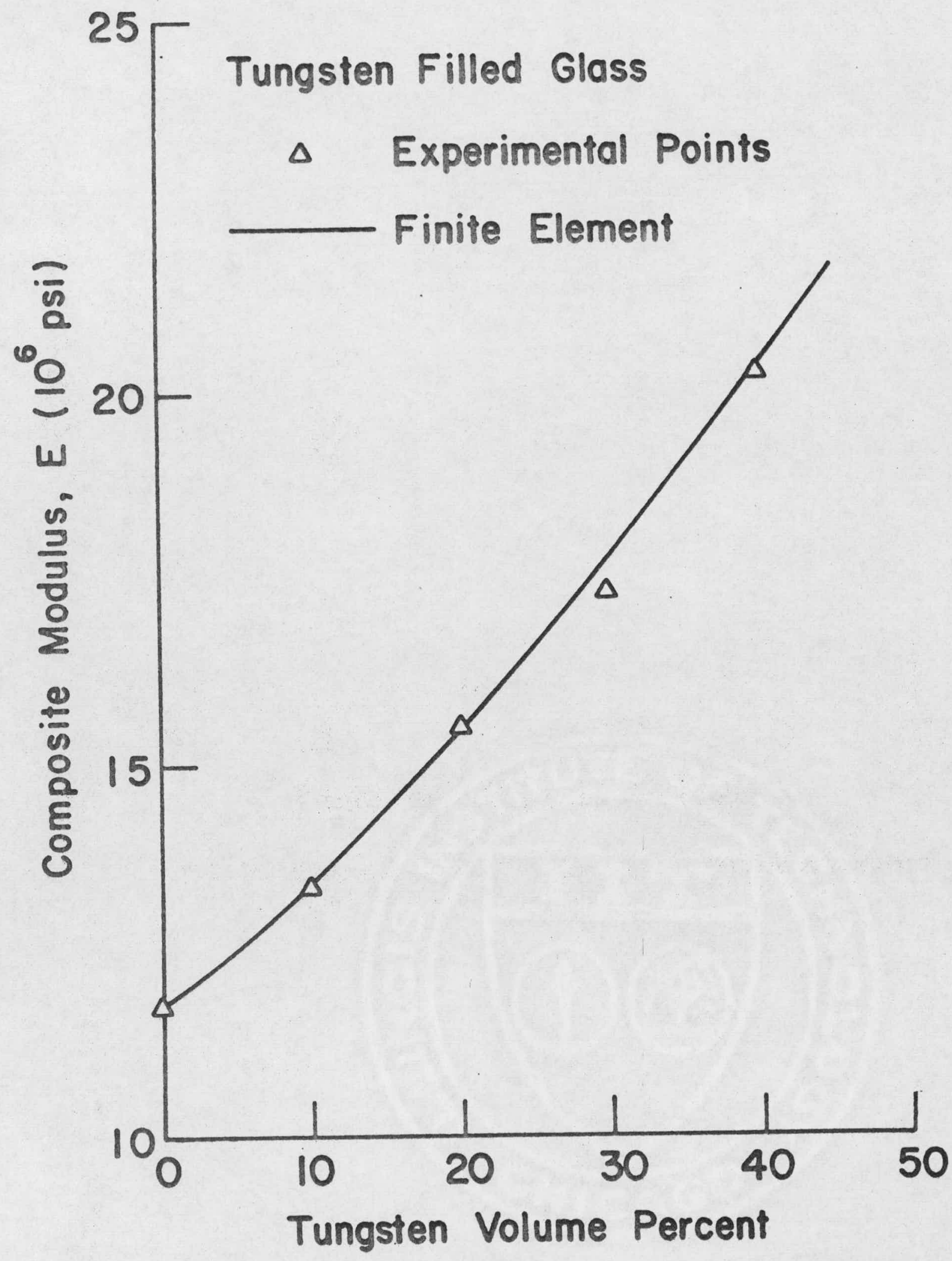

Fig. 18. Comparison of Predicted Modulus of Elasticity of TungstenFilled Glass with Experimental Data of Reference 15. 
Table 2. Predicted Properties of Porous and Filled Ceramics

\begin{tabular}{|c|c|c|c|c|c|c|c|}
\hline \multirow[b]{3}{*}{$\begin{array}{l}\text { Vol. Fraction } \\
\text { Filler or Pores }\end{array}$} & \multirow{3}{*}{$\begin{array}{l}\text { Relative } \\
\text { Modulus } \\
(E / E o)^{*}\end{array}$} & \multirow[b]{3}{*}{$\begin{array}{l}\text { Poisson's } \\
\text { Ratio }\end{array}$} & \multicolumn{3}{|c|}{ GLASS ALUMINA } & \multicolumn{2}{|c|}{ GLASS TUNGSTEN } \\
\hline & & & \multicolumn{2}{|c|}{$\begin{array}{c}\text { Modulus of Elasticity } \\
\left(10^{6} \mathrm{psi}\right)\end{array}$} & \multirow[b]{2}{*}{$\begin{array}{l}\text { Poisson's } \\
\text { Ratio }\end{array}$} & \multirow{2}{*}{$\begin{array}{l}\text { Modulus of } \\
\text { Elasticity } \\
\left(10^{6} \text { psi) }\right.\end{array}$} & \multirow[b]{2}{*}{$\begin{array}{c}\text { Poisson's } \\
\text { Ratio }\end{array}$} \\
\hline & & & $\begin{array}{l}\text { Finite } \\
\text { Element. }\end{array}$ & $\begin{array}{l}\text { Lower } \\
\text { Bound** }\end{array}$ & & & \\
\hline 3.04 & 0.932 & 0.255 & 12.3 & 12.3 & 0.198 & 12.3 & 0.197 \\
\hline 8.33 & 0.859 & 0.232 & 13.2 & 13.2 & 0.199 & 13.1 & 0.196 \\
\hline 15.54 & 0.753 & 0.222 & 14.7 & 14.7 & 0.197 & 14.5 & 0.192 \\
\hline 24.30 & 0.639 & $0.20 \overline{8}$ & 16.7 & 16.6 & 0.193 & 16.3 & 0.186 \\
\hline 38.58 & 0.469 & 0.178 & 20.7 & 20.3 & 0.186 & 19.9 & 0.175 \\
\hline 43.83 & 0.410 & 0.163 & 22.4 & 22.0 & 0.184 & 21.4 & 0.172 \\
\hline
\end{tabular}

* $E_{0}=$ matrix modulus; $E=$ composite modulus.

** Hashin-Shtrikman theory (Ref. 3). 


\section{Effect of a Weak Interface on Composite Properties}

In an actual composite material, the properties of the material at the interface may be different from those of the filler and matrix. Continuous displacements at the interface imply perfect bonding between the filler particles and the matrix. When perfect bonding does not exist between the filler and the matrix, behavior of the interface should be simulated by assigning different property values to the material at the interface. This is very easily accomplished using the axisymmetric finite element method. The shaded elements in Figs. 19 and 20 have been assumed to represent the finite thicknesses of the interface for filler contents of 3.04 and 24.30 percents respectively. The shaded elements account for 0.48 percent of the total volume in the former case and 3.02 percent in the latter case. A close examination of the finite elements in Fig. 7 reveals that a much finer grid is needed to study the effect of the interface at a high volume fraction of the filler. The modulus of elasticity assigned to the elements at the interface was 1000 psi which is very small compared to that of the matrix $\left(E=11.8 \times 10^{6} \mathrm{psi}\right)$ or the filler $(E=$ $\left.60.4 \times 10^{6} \mathrm{psi}\right)$. This represents the case of a very weak interface.

The weak interface as described above completely changes the stress distribution around the interface. The stresses in the elements (in the matrix) adjacent to the interface have been plotted in Figs. 21 and 22. A very small magnitude of radial stress around the interface indicates a free boundary. The curves for tangential stresses with finite interface are very similar to the ones obtained for stresses around the cavity as shown in Figs. 9 and 10. Due to very low modulus, the interface is not able to transfer any stress from the matrix to the hard inclusions and therefore represents a case of the filler particles completely debonded from the matrix. The hard inclusions with weak interface carry very low stresses and hence do not contribute to the enhancement of the modulus of the composite. This is similar to what Stett and Fulrath ${ }^{(11)}$ have described as 


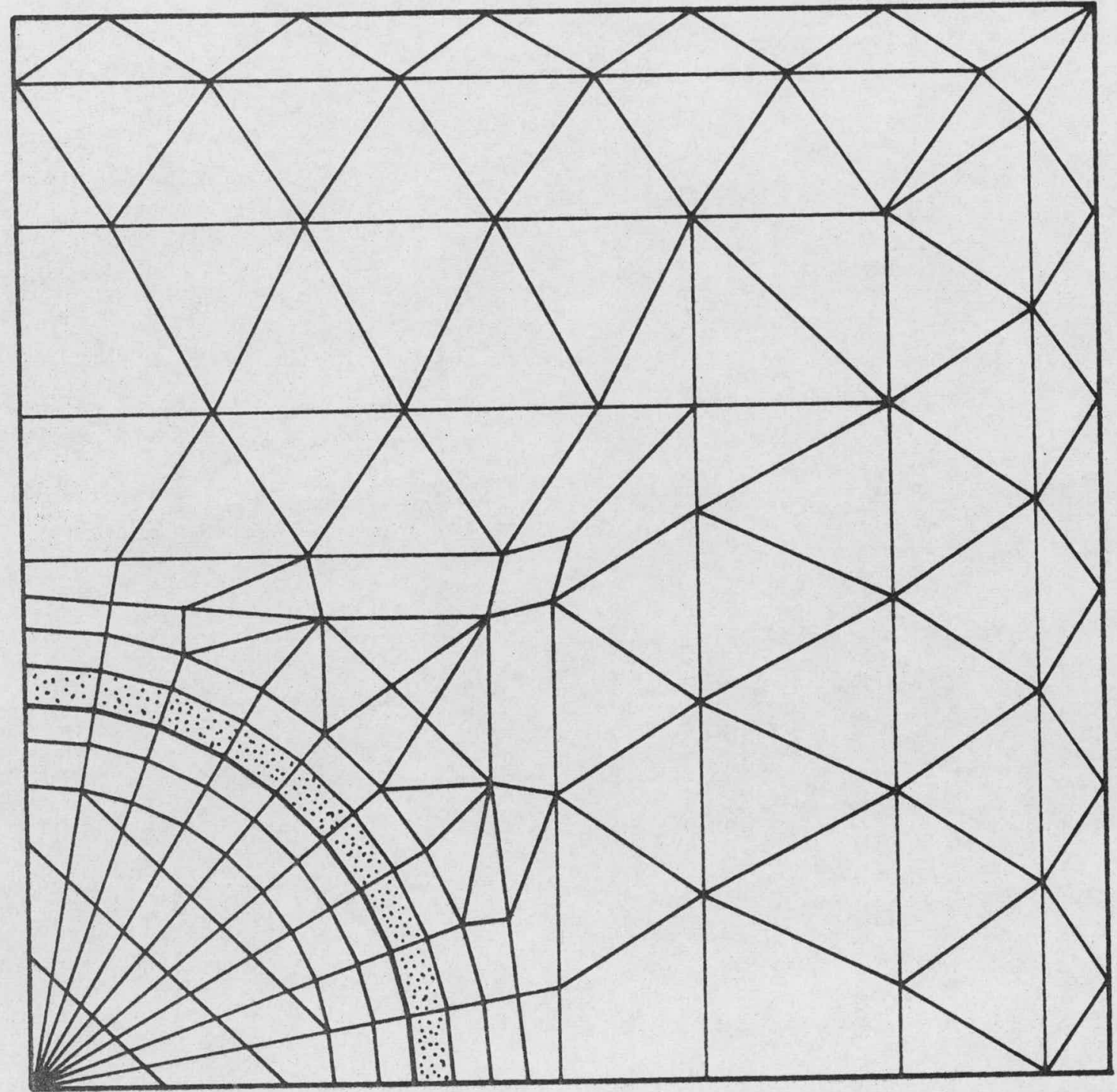

Fig. 19. Shaded Elements Represent Finite Thickness of the Interface for $r_{2} / r_{1}=0.357$ 


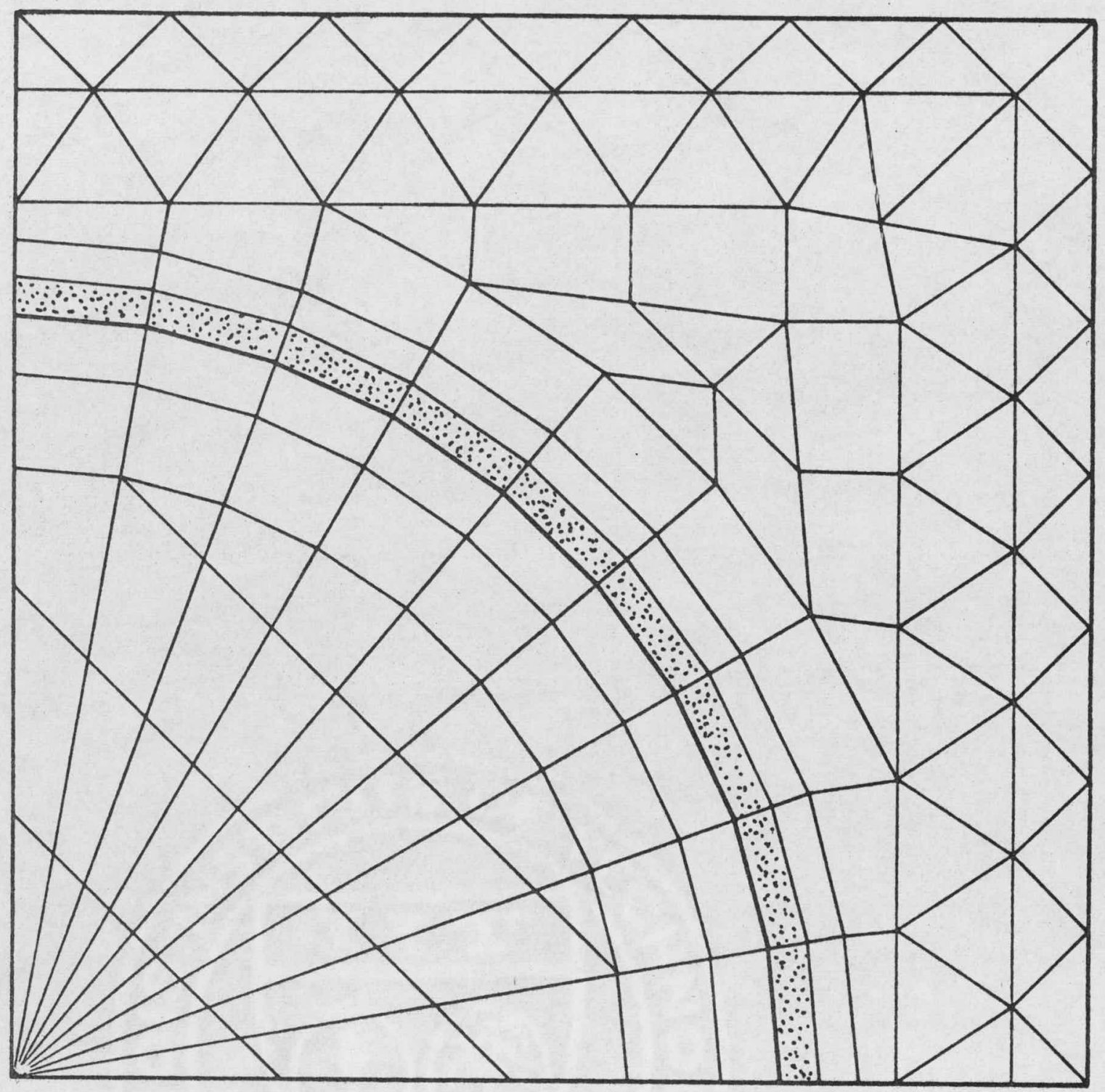

Fig. 20. Shaded Elements Represent Finite Thickness of the Interface for $r_{2} / r_{1}=0.714$ 


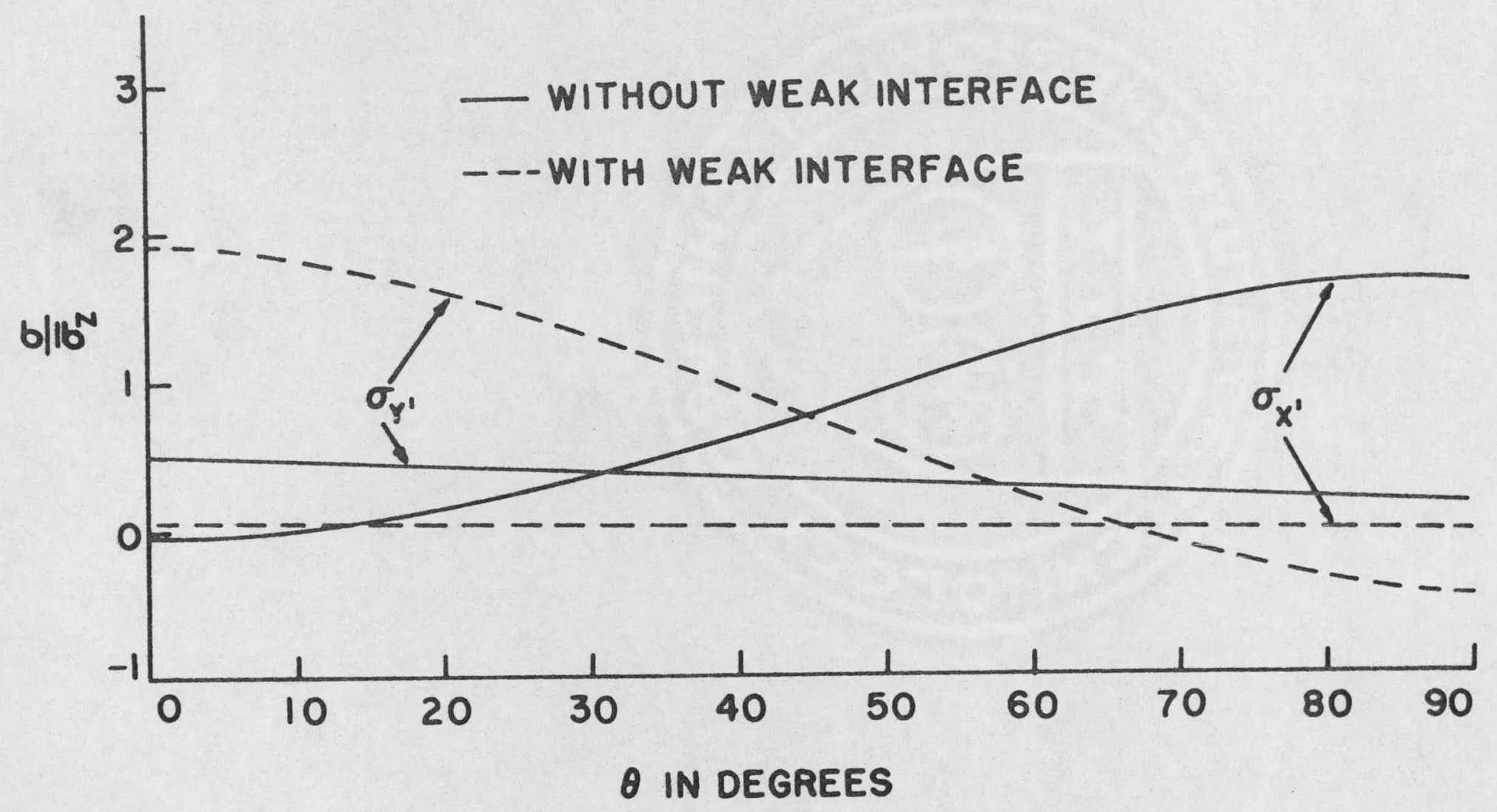

Fig. 21. Comparison of Matrix Stresses With and Without a Weak Interface in a Composite $\left(r_{2} / r_{1}=0.357\right)$ 


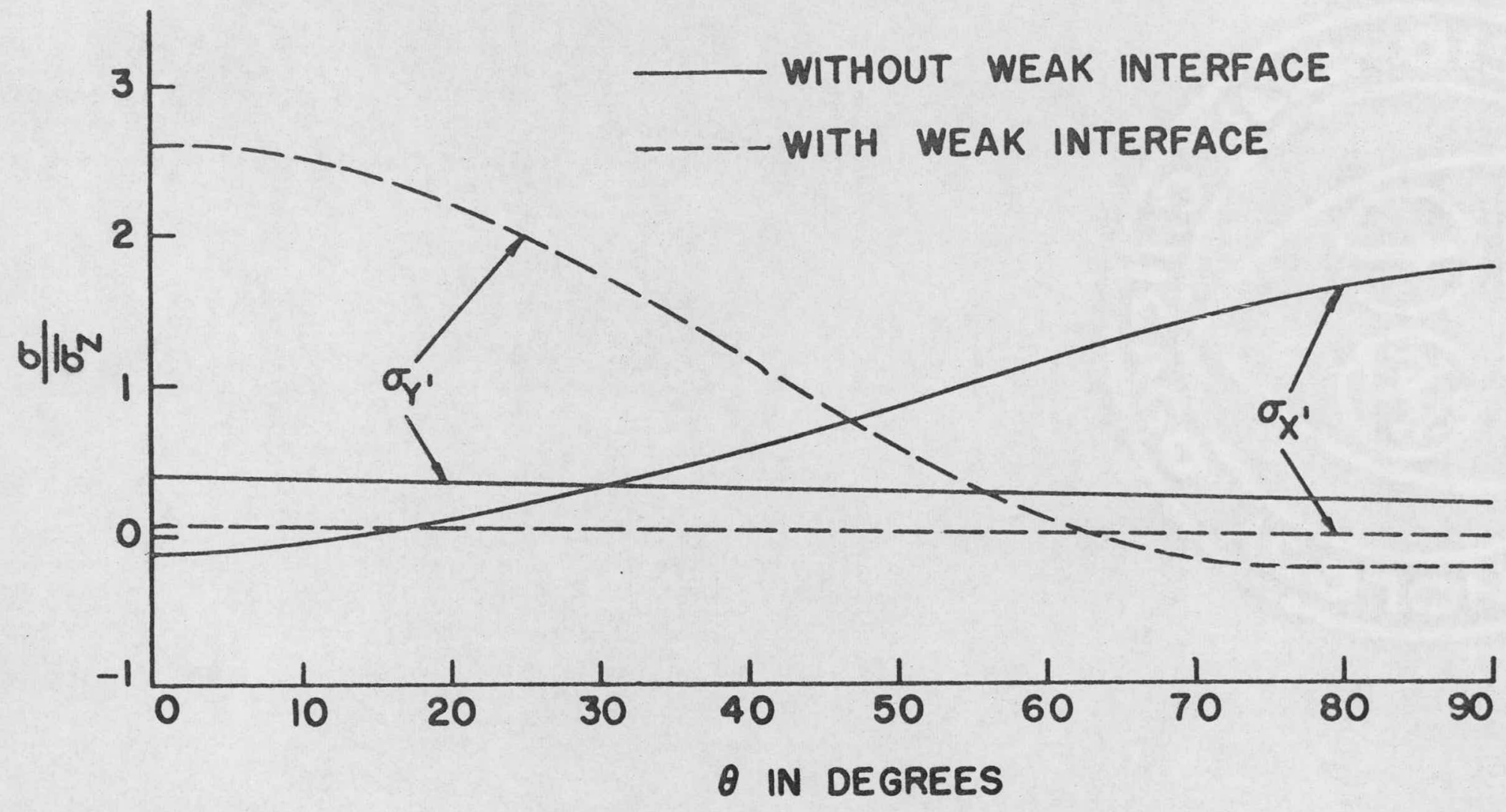

Fig. 22. Comparison of Matrix Stresses With and Without a Weak Interface in a Composite $\left(r_{2} / r_{1}=0.714\right)$ 
pseudoporosity which results in the weakening of the composite. The modulus of elasticity of the composite decreases with higher filler contents as indicated in Table 3.

Table 3. Effect of Interface on Modulus of Elasticity of Composite

\begin{tabular}{ccc}
\hline $\begin{array}{c}\text { Volume Fraction } \\
\text { Filler }\end{array}$ & \multicolumn{2}{c}{ Composite Modulus (psi) } \\
Without Interface & With Interface \\
\hline 3.04 & $12.3 \times 10^{6}$ & $11.05 \times 10^{6}$ \\
24.30 & $16.7 \times 10^{6}$ & $6.97 \times 10^{6}$ \\
43.83 & $22.4 \times 10^{6}$ & $3.84 \times 10^{6}$ \\
\hline
\end{tabular}




\section{THREE DIMENSIONAL ANALYSIS OF PARTICULATE COMPOSITES}

\section{Introduction}

It was pointed out in Section II that axisymmetric representation of the composite only approximates its real packing and structure. The axisymmetric cells are not an actual repetitive unit but are related in their dimensions to the interparticle spacings. They do not account for the total volume of the composite as shown in Fig. 8. The volume fractions of filler are calculated by assuming an appropriate packing of the axisymmetric cells and each arrangement gives a different number as indicated in Table 1. A computer program which analyzes three dimensional solids without restrictions enables one to overcome the apparent major limitation on the use of an axisymmetric analysis of the composite materials.

The program, SAFE-3D(19), for the three-dimensional elastic analysis of heterogeneous composite structures was used in the analysis discussed in this Section. This program uses the following types of finite elements:

1) tetrahedral elements to represent the continuum

2) triangular plane stress membrane elements to represent inner liner or outer case, and

3) uniaxial tension-compression elements to represent internal reinforcement as shown in Fig. 23.

The structure can be of arbitrary geometry and can have any desired distributions of material properties, temperatures, surface loadings, and boundary conditions. The generality of the SAFE-3D program puts a limitation on its use from an economical standpoint.

To simplify the geometric subdivision of the solids, the tetrahedral elements are placed into groups of three, which together form an octahedron (eight-sided figure). In a uniform mesh the octahedron may look like a triangular prism, as 

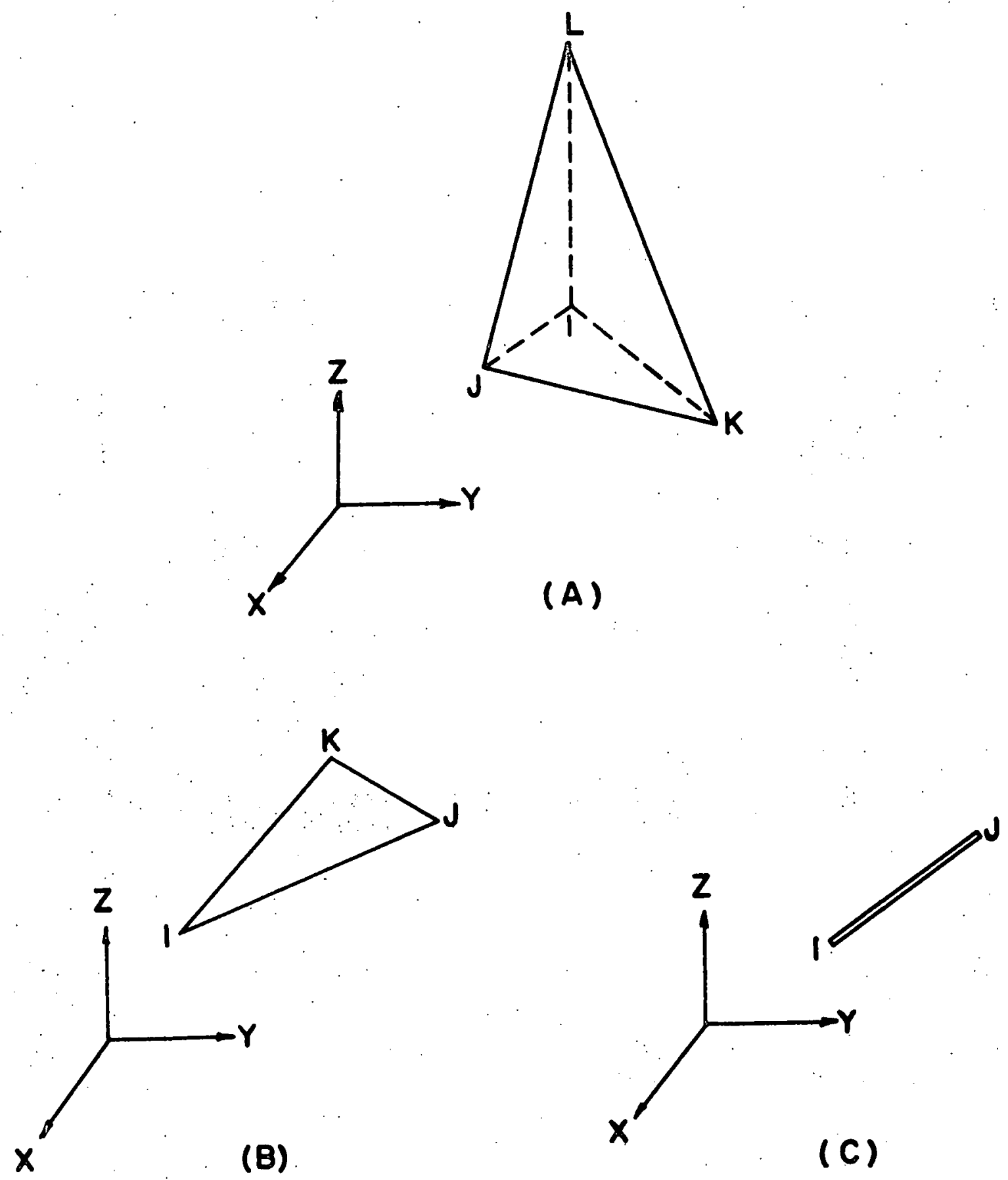

Fig. 23. 'Finite Element Types: (A) Tetrahedral Element, (B) Membrane Plane Element, and (C) Uniaxial Bar Element 
shown in Fig. 24(a). The octahedron becomes the input element, and it is internally subdivided by the program into three tetrahedra, as shown in Fig. 24(a). At irregular regions in the solids, the octahedral elements may degenerate to elements of one or two tetrahedra as shown in Fig. 24(b) and (c).

\section{Boundary Conditions}

Stresses in three-dimensions were calculated around spherical inclusions for various interparticle spacings. Spherical inclusions were assumed packed in a cubic array as shown in Fig. 25. As in the case of the axisymmetric analysis, the stress-strain relations of the martix and the filler were assumed elastic and also perfect bonding was assumed between the filler and matrix.

Due to the symmetry of the problem, one needs to analyze only one eighth of the sphere embedded in a cube as shown in Fig. 26. Three-dimensional meshes (subdivisions) of the solid for the cases $r_{2} / r_{1}=0.357,0.714,0.870$ and 0.952 (where $r_{1}$ and $r_{2}$ are shown in Fig. 26) are shown in Figs. 27-30. It was assumed that the composite is strained in the $z$-direction and that no tractions are applied in the $x$ or $y$ direction. The following boundary conditions for the typical region (Fig. 26) have to be satisfied.

By symmetry, the shear stresses on all the faces of the cube $A B C D E F G H$ are:

$$
\tau_{x y}=\tau_{y z}=\tau_{z x}=0
$$

The faces $A B F E, E F G H$ and $B C G F$ of the cube remain parallel to their original positions after they are displaced by the force in the z-direction whereas the normal displacement of the faces $A B C D, A D H E$ and $D C G H$ is zero. The normal forces on the faces $A B F E$ and BCGF must be zero so that:

$$
\begin{array}{ll}
\int_{A} d A=0 & \text { on } x=r_{1} \\
\int_{A} \sigma_{y} d A=0 & \text { on } y=r_{1}
\end{array}
$$




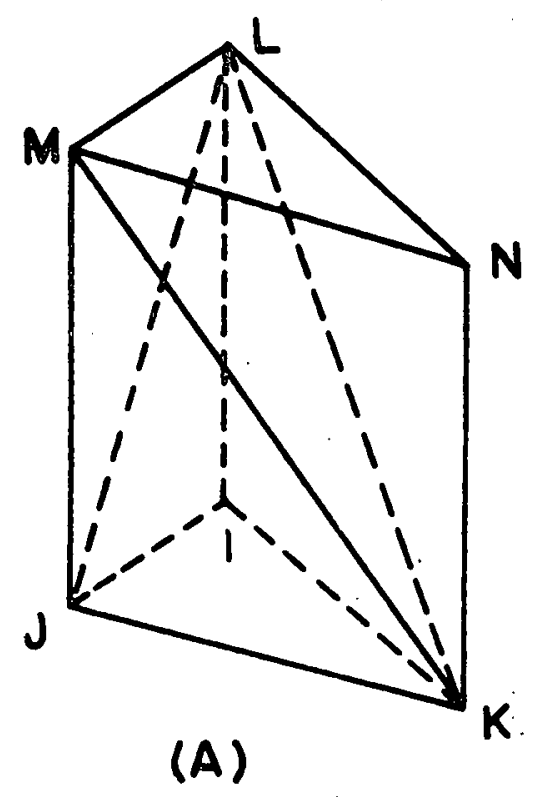

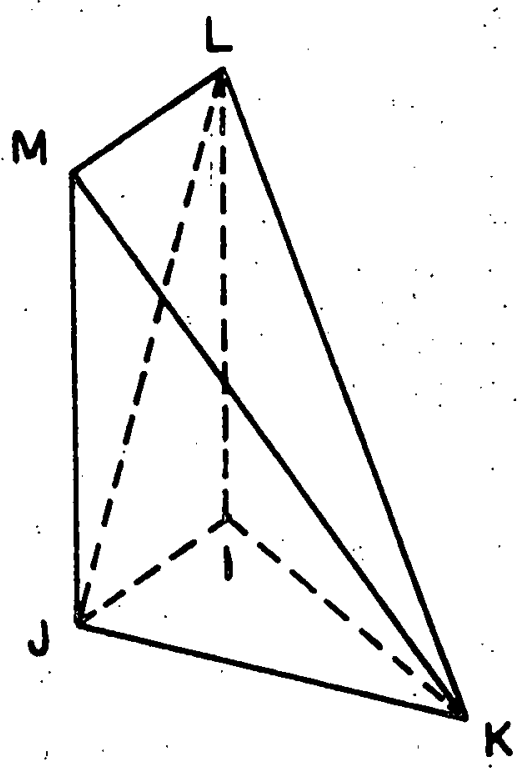

(B)

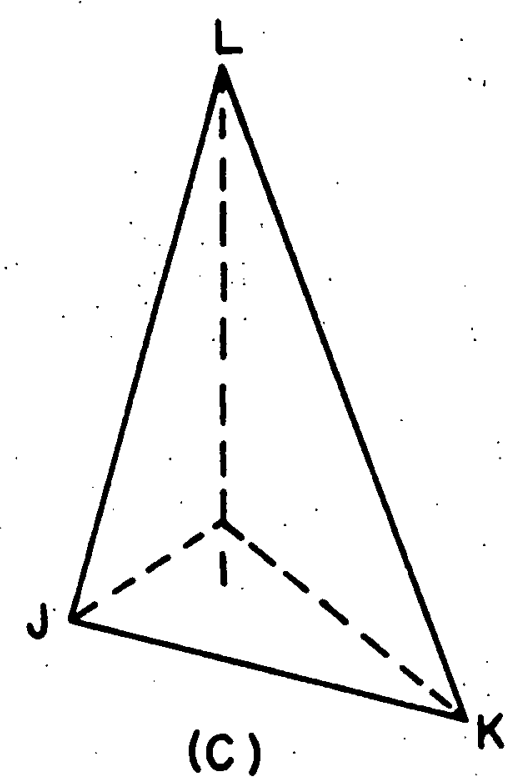

(C)

Fig. 24. Octahedral Element and Degenerate Cases: (A) Basic Octahedral Element, (B) Two Tetrahedra Degenerate Case, and (C) One Tetrahedron Degenerate Case 


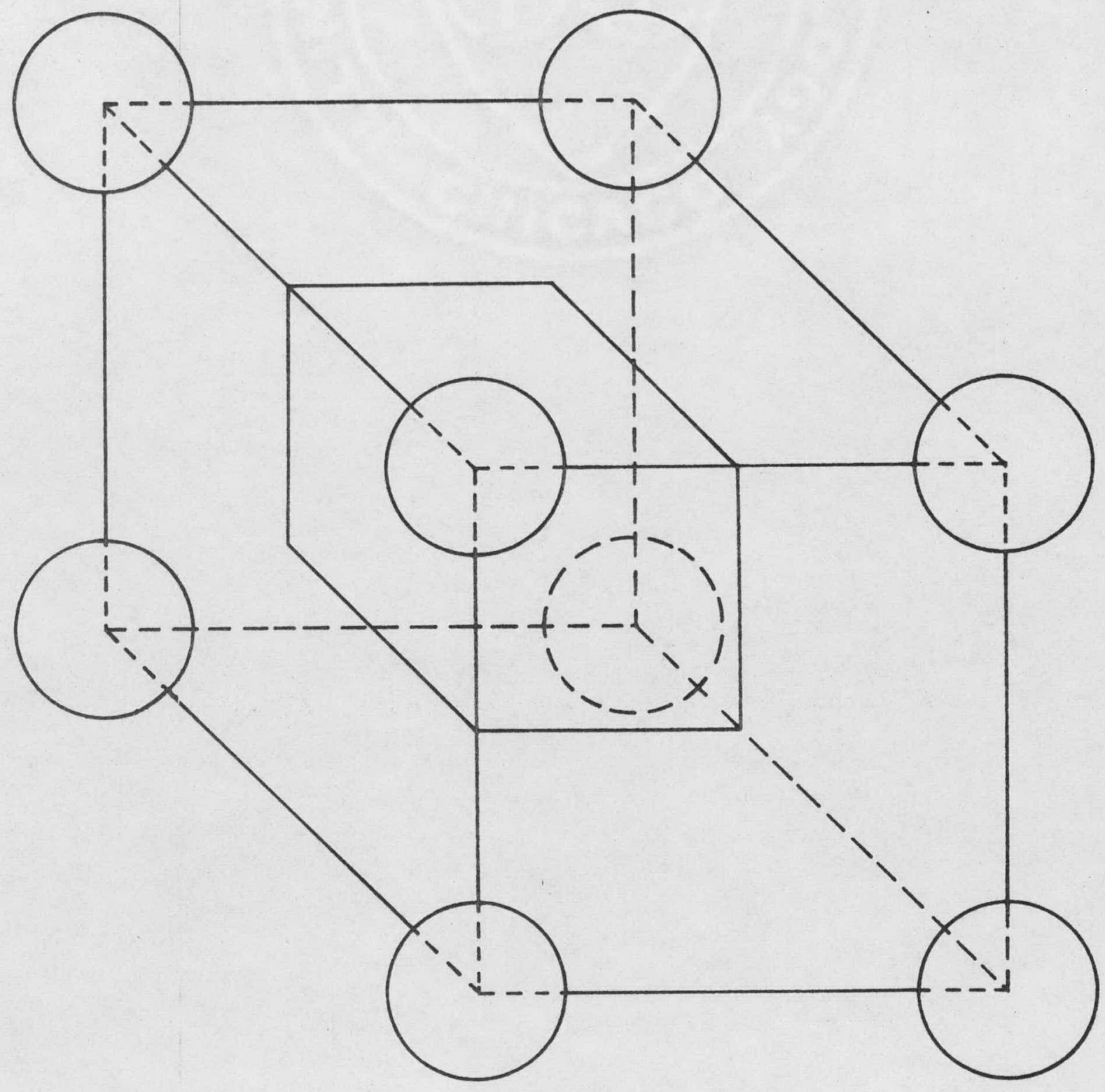

Fig. 25. Packing of Spherical Inclusions in a Composite 


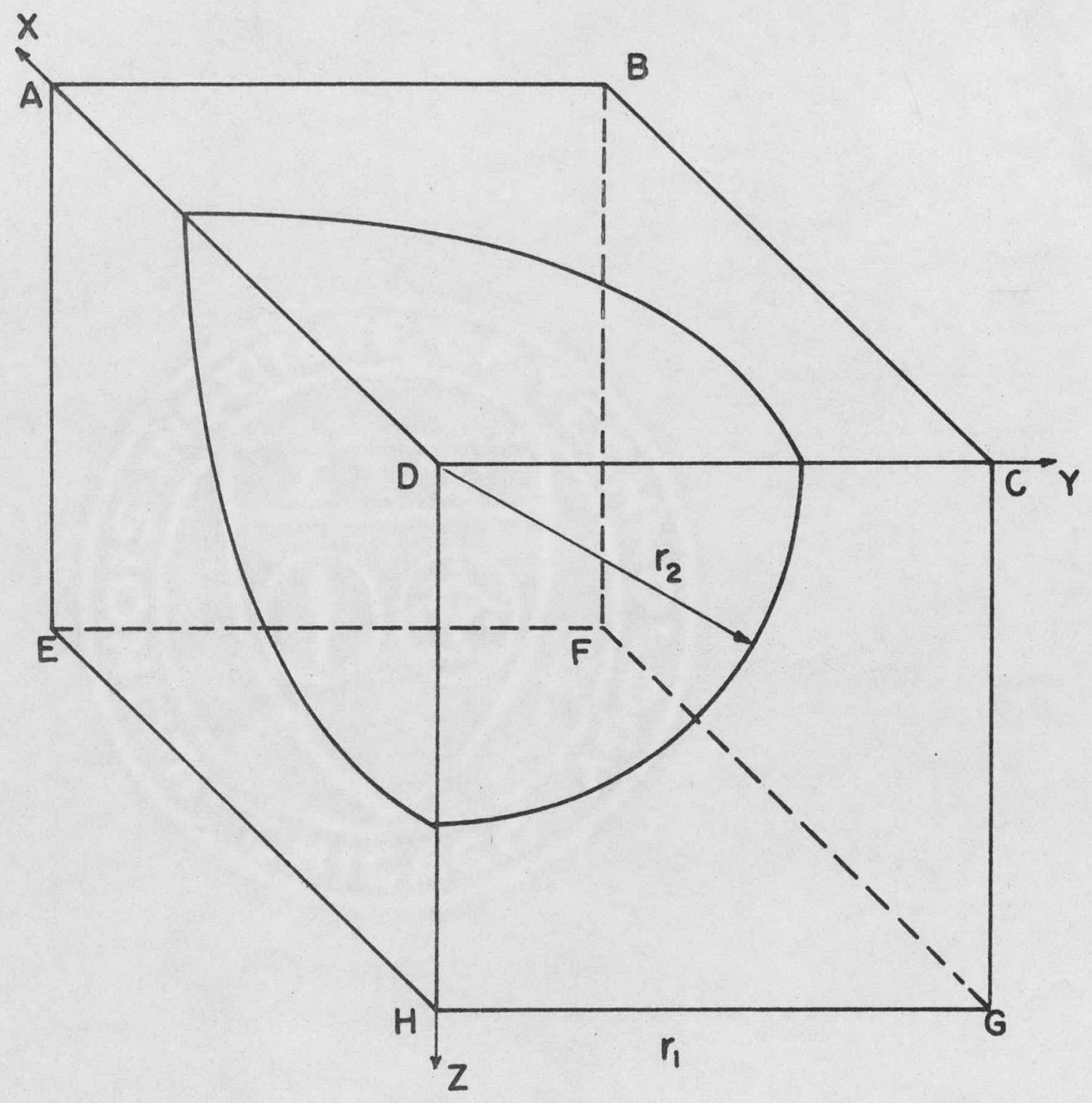

Fig. 26. A Typical Three-Dimensional Region Analyzed 


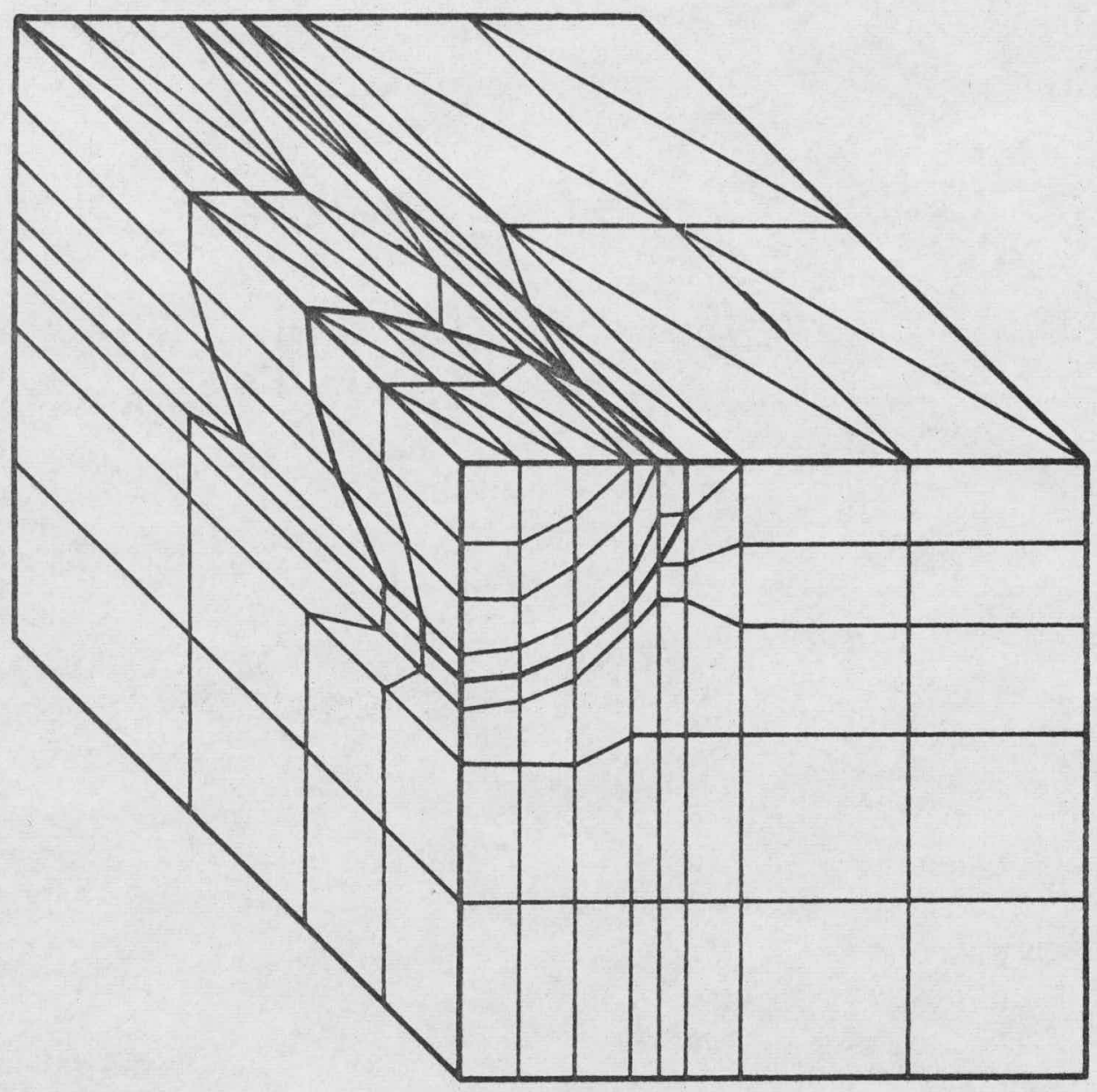

Fig. 27. Three-Dimensional Mesh for $r_{2} / r_{1}=0.357$ 


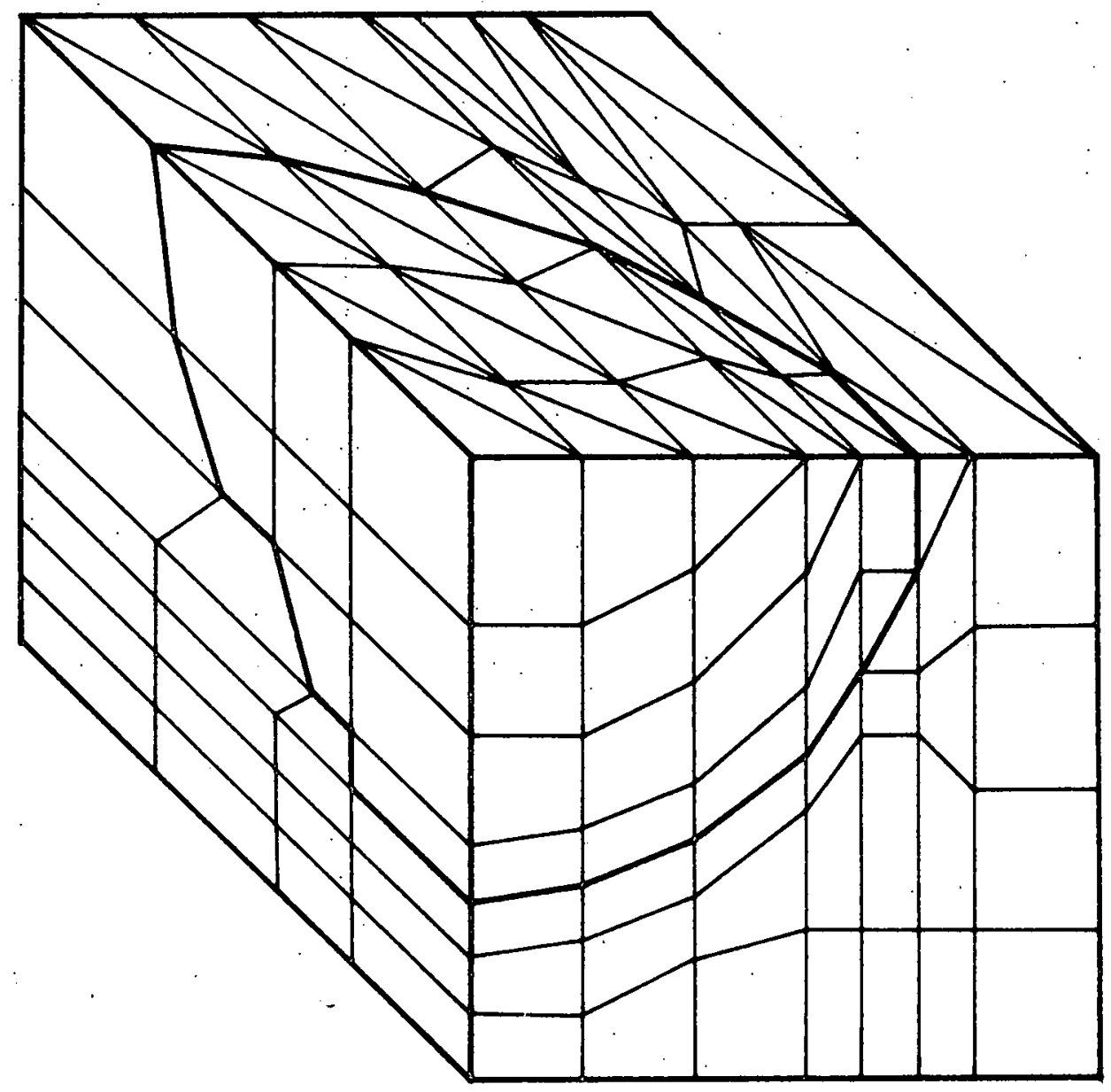

Fig. 28. Three-Dimensional Mesh for $r_{2} / r_{1}=0.714$ 


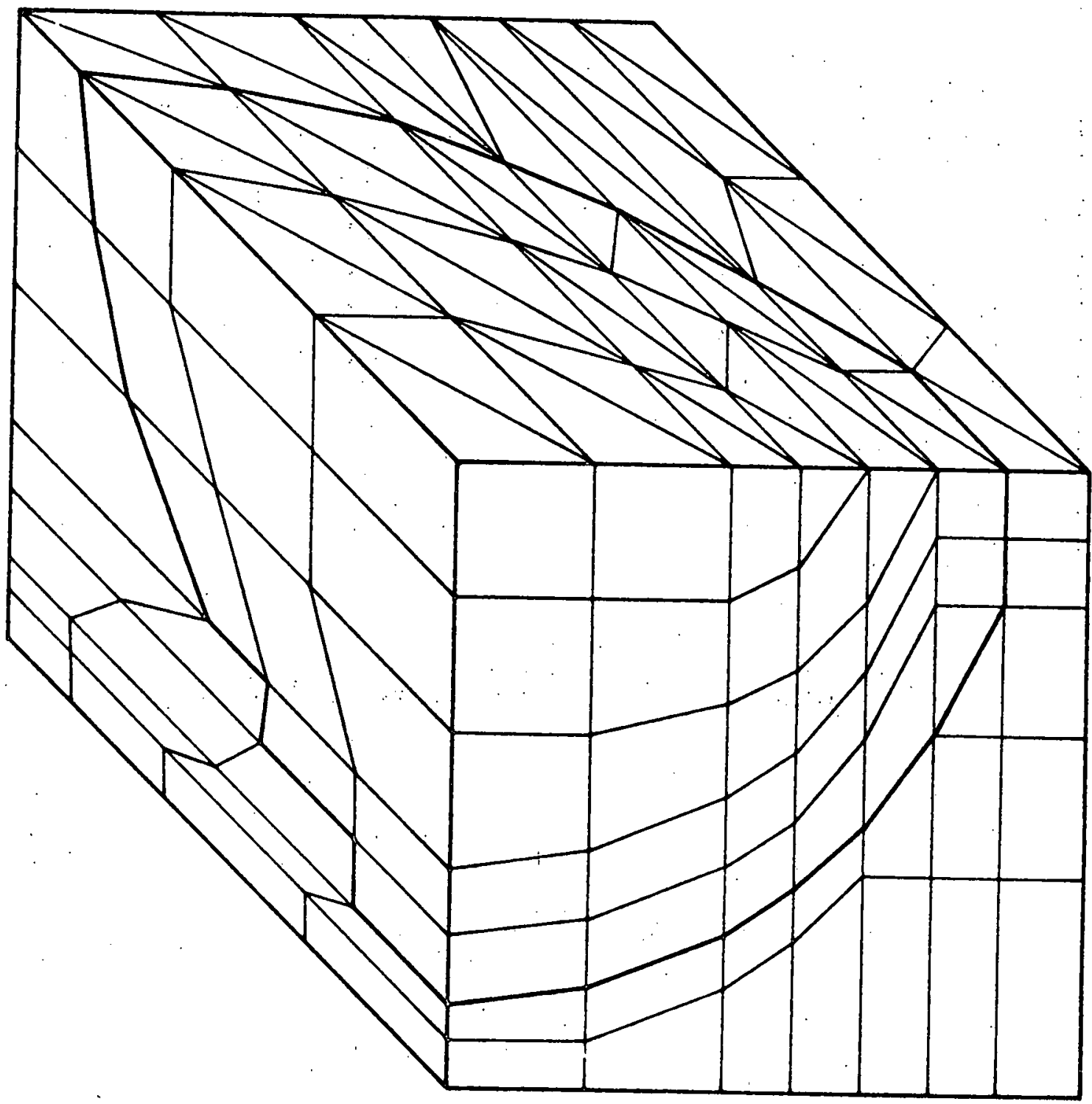

Fig. 29. Three-Dimensional Mesh for $r_{2} / r_{1}=0.870$ 


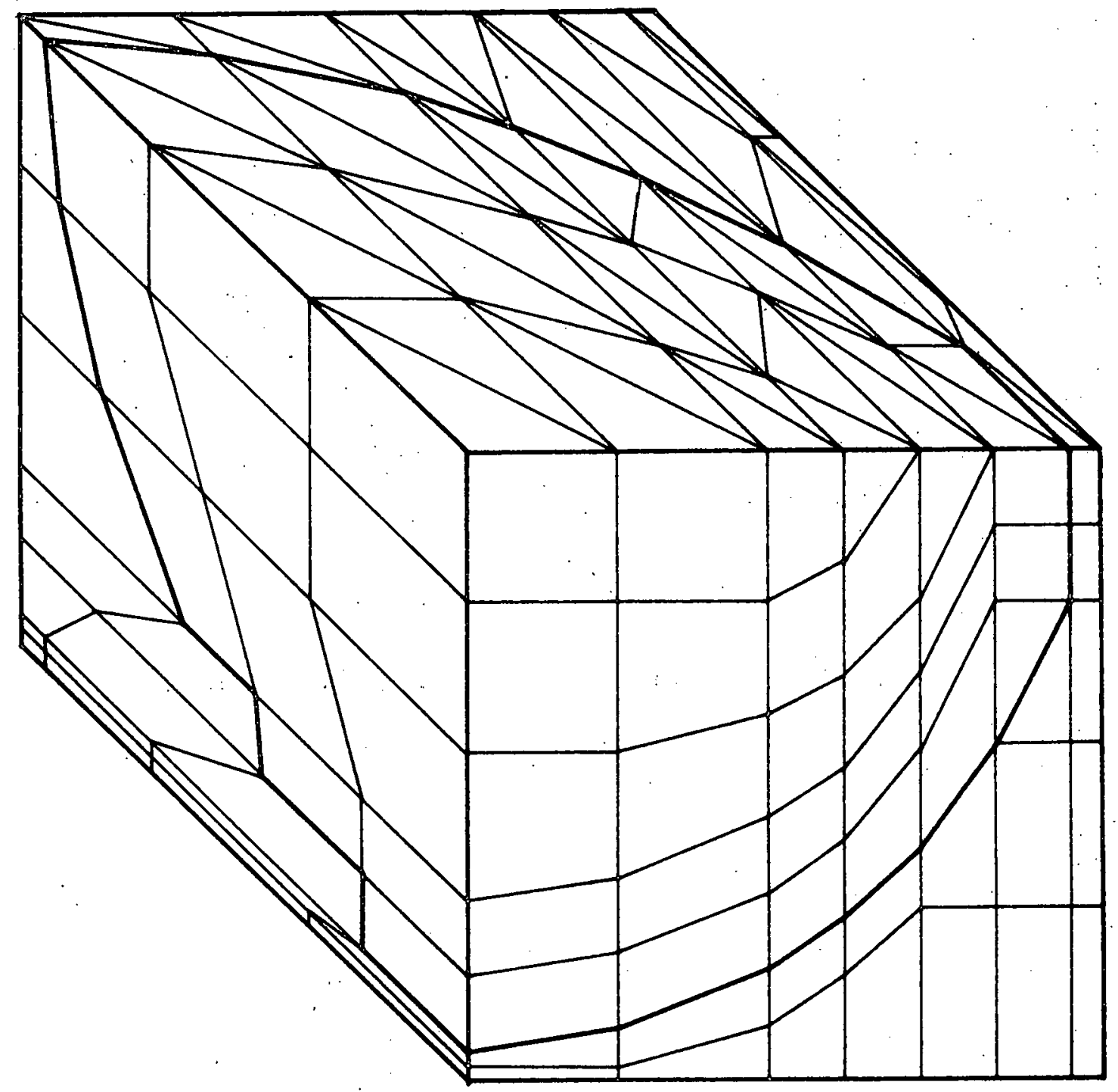

Fig. 30. Three-Dimensional Mesh for $r_{2} / r_{1}=0.952$ 
where the integral is replaced by a summation in the finite element method. These boundary conditions are equivalent to the ones used previously for an axisymmetric representation of the composite material.

The above boundary conditions were satisfied by a superposition method. This superposition method and subsequent calculation of composite modulus of elasticity and Poisson's ratio are discussed in Appendix .

\section{Internal Stresses}

The internal triaxial stresses were calculated throughout the volume of representative filled ceramics with filler contents up to 45.23 percent (minimum interparticle spacing $=0.096 r_{2}$, where $r_{2}=$ radius of spherical particles)

The following component properties were assumed:

$$
\begin{array}{ll}
\text { Glass matrix } & E=11.8 \times 10^{6} \mathrm{psi} \\
\text { Alumina filler } & \nu=0.197 \\
& E=60.4 \times 10^{6} \mathrm{psi} \\
\nu & =0.257
\end{array}
$$

These properties are the same as used in Section II and equivalent to the experimental values reported by Hasse Iman and Fulrath ${ }^{(14)}$.

The stresses around the spherical inclusions are shown in Figs. 31-34 for four different interparticle spacings $\frac{r_{2}}{r_{1}}=0.357,0.714,0.870$ and 0.952$)$. The stresses are represented as a ratio $\sigma / \bar{\sigma}_{z}$ where $\bar{\sigma}_{z}$ is the average stress applied to the composite. Thus, the ratio represents the stress concentration around the cavity. The stresses are in the elements adjacent to the inclusions and are assumed to act at the center of each of the elements.

The radial and tangential stresses obtained in the axisymmetric analysis of filled ceramics have been compared with the results of the three-dimensional analysis for three different interparticle spacings $\left(\frac{r_{2}}{r_{1}}=0.357,0.714\right.$ and 0.870$)$. 


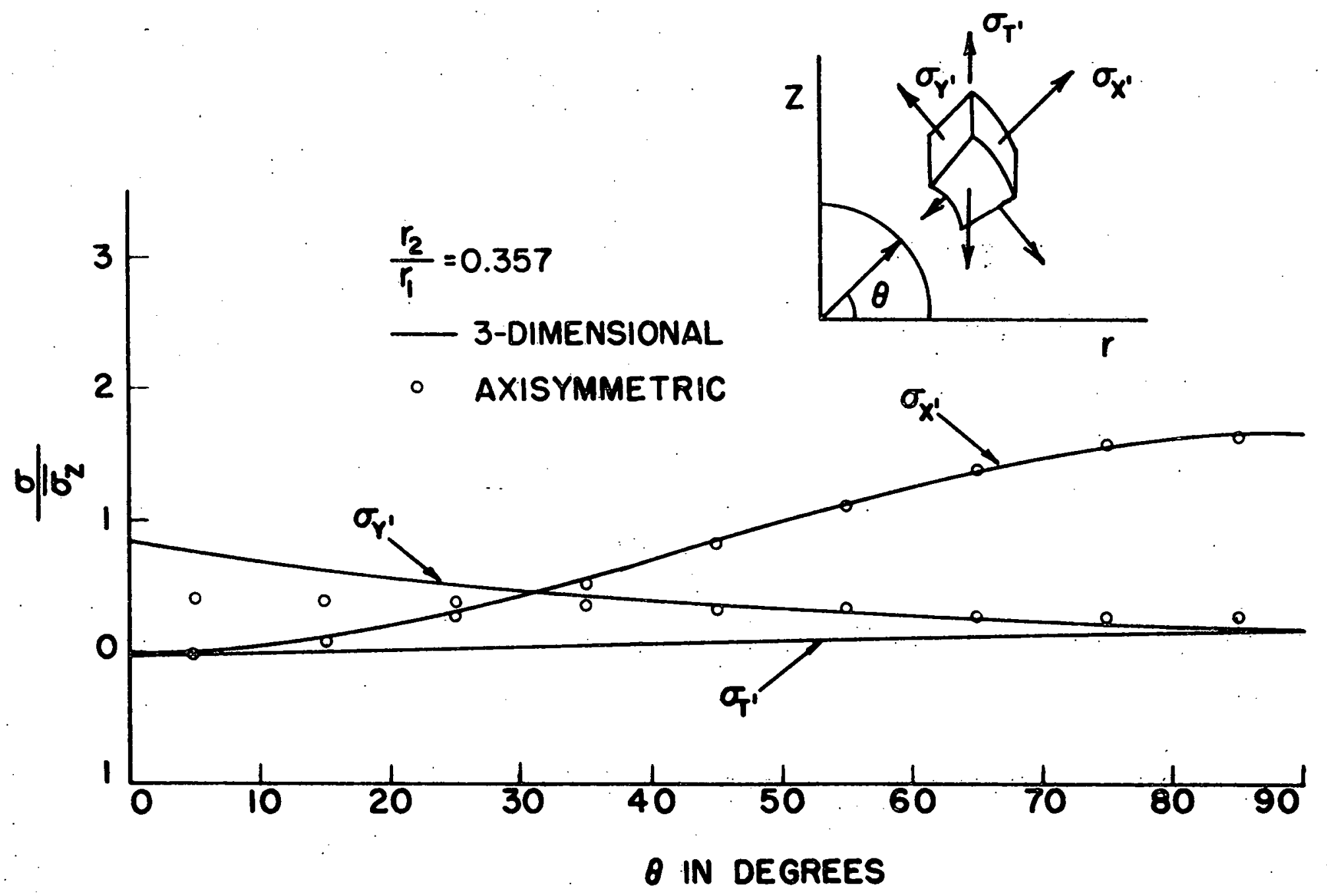

Fig. 31. Matrix Stresses af Interface in Alumina-Filled Glass with $r_{2} / r_{1}=0.357$ 


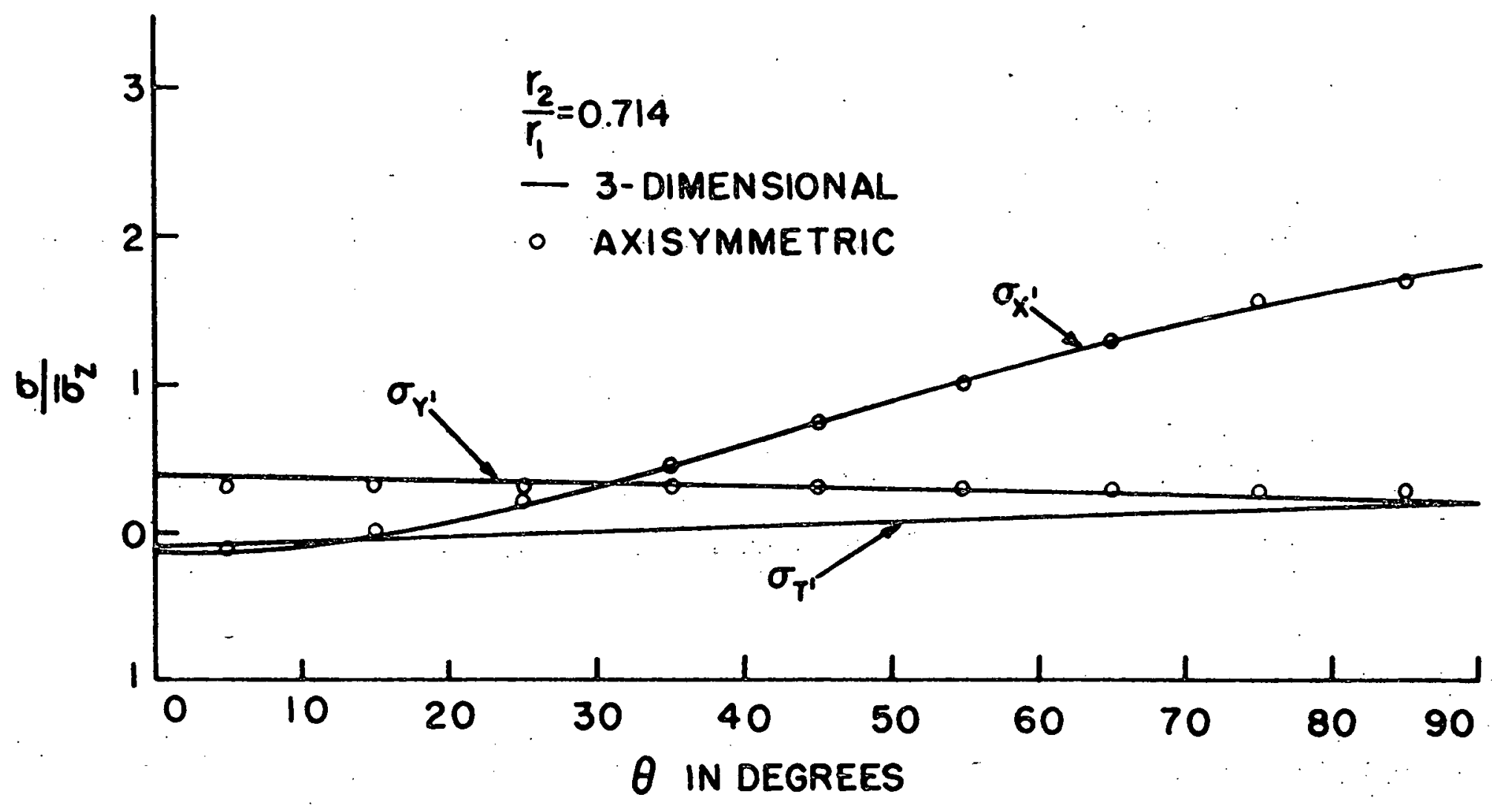

Fig. 32. Matrix Stresses at Interface in Alumina-Filled Glass with $r_{2} / r_{1}=0.714$ 


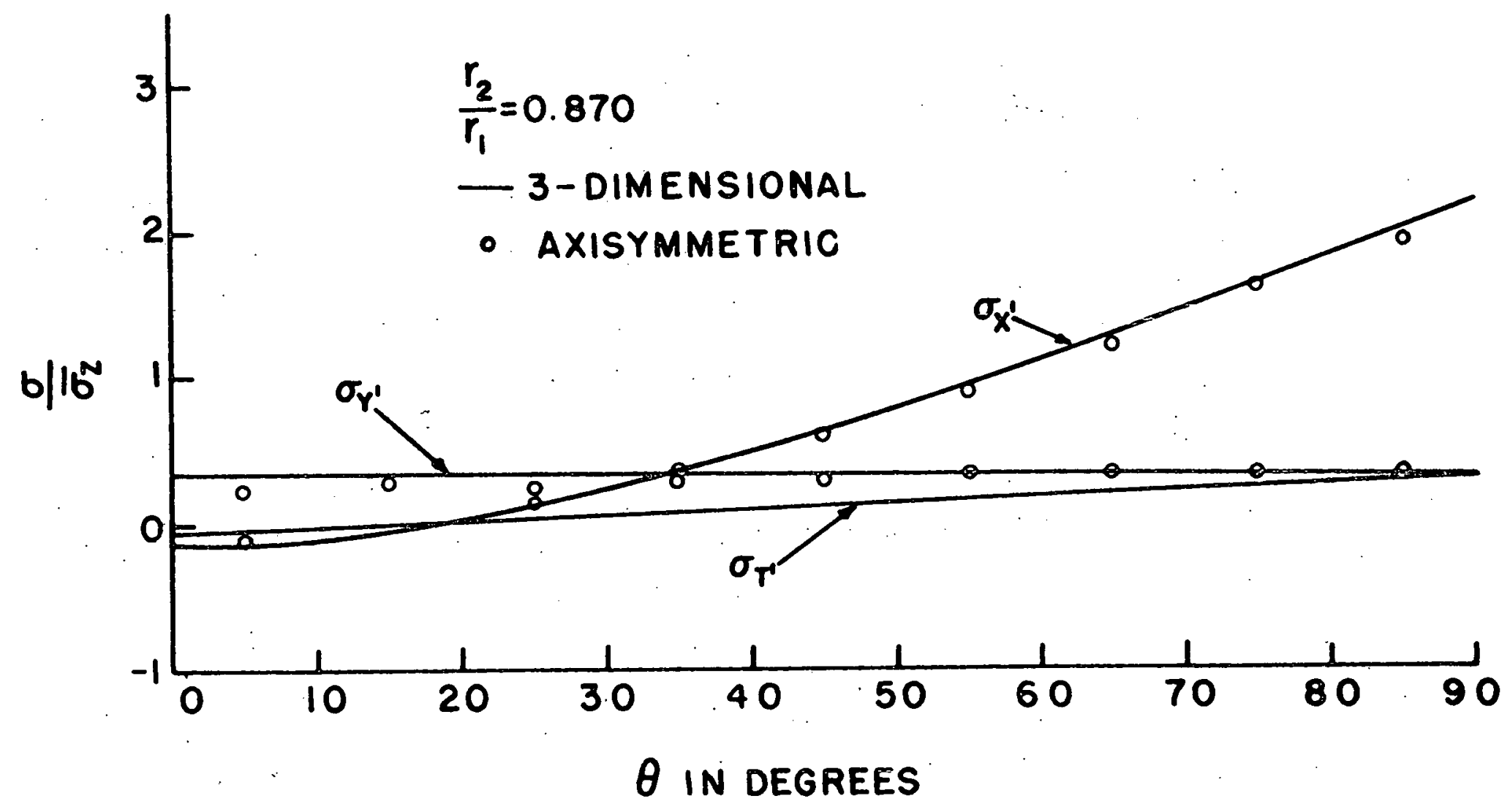

Fig. 33. Matrix Stresses at Interface in Alumina-Filled Glass with $r_{2} / r_{1}=0.870$ 


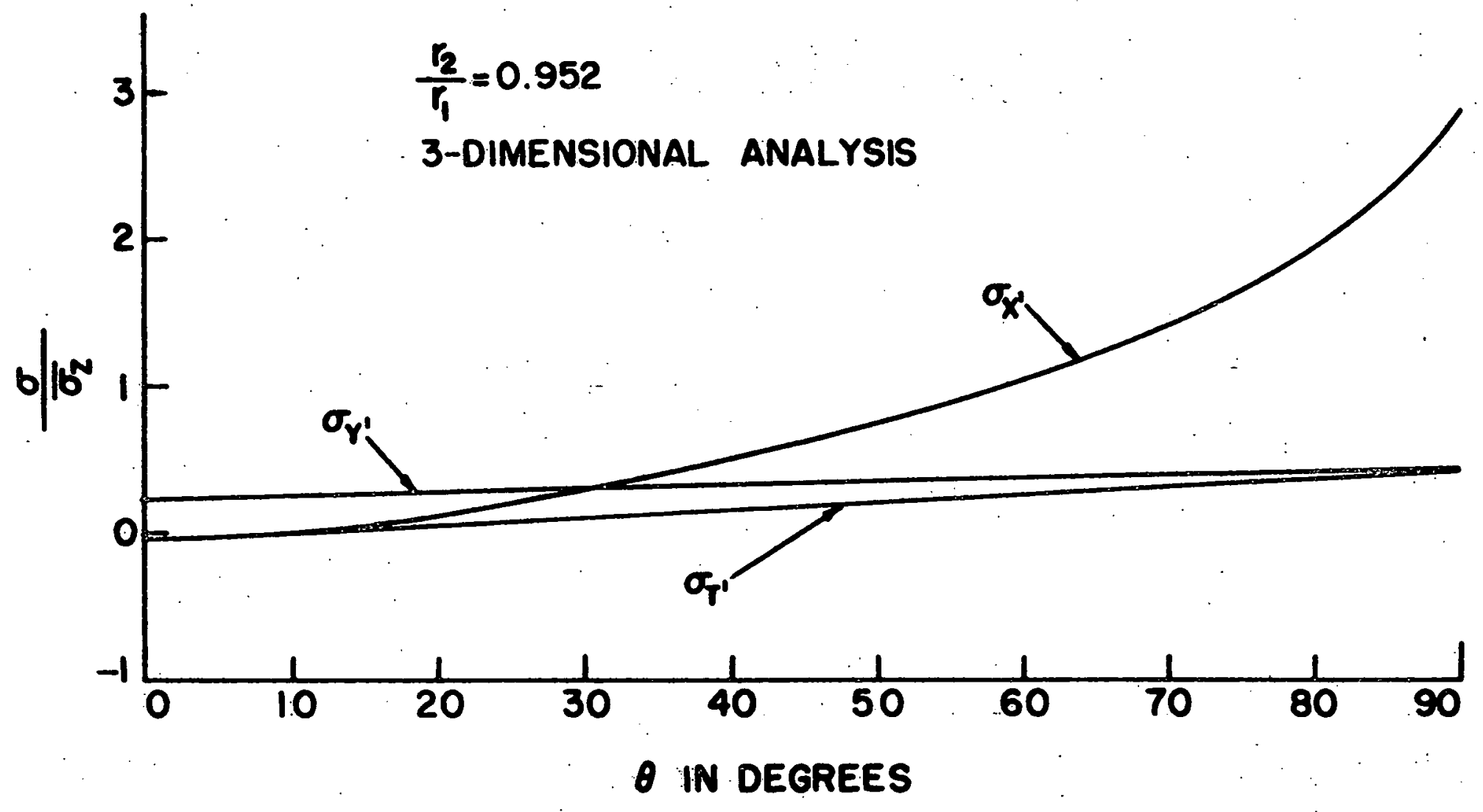

Fig. 34. Matrix Stresses at Interface in Alumina-Filled Glass with $r_{2} / r_{1}=0.952$ 
Figs. 31-33 stiow that the two analyses give almost identical stress distributions. Minor differences at some places may be attributed to the shape and size of the finite elements. The stresses for the case $\frac{r_{2}}{r_{1}}=0.952$ (Fig. 34) show the same general trend of the stresses around the inclusion, and as would be expected the radial stress at $\theta=90^{\circ}$ is much higher compared to that for the other three cases.

The variation of stresses at the pole $\left(\theta=90^{\circ}\right)$ and the equator $\left(\theta=0^{\circ}\right)$ of spherical particles are shown in Fig. 35 as a function of $r_{2} / r_{1}$ (which represents a definite interparticle spacing). The curves were extrapolated to $r_{2} / r_{1}=0$ which corresponds to a single inclusion in an infinite matrix. Theoretical results of Goodier $^{(1)}$ applicable for $r_{2} / r_{1}=0$, agree quite well with the predicted values. Also shown in Fig. 35 are the stresses obtained by the axisymmetric analysis. The results of the two analyses are quite comparable in all cases except for tangential stresses at $\theta=0^{\circ}$. In the axisymmetric analysis, at $\theta=0^{\circ}$ the stresses are independent of position around the equator. This is not true for the three-dimensional analysis. Thus, the averaging effect is probably the reason for the discrepancy in the tangential stresses.

Elastic Constants

The predicted moduli of elasticity are shown in Fig. 36 and Table 4 as a function of the ratio $r_{2} / r_{1}$. The modulus of elasticity increases as the filler content increases. The moduli of elasticity values predicted by axisymmetric analysis have also been plotted in Fig. 36. It is seen, once again, that the results of the two analyses are quite close when plotted as a function of interparticle spacing. 


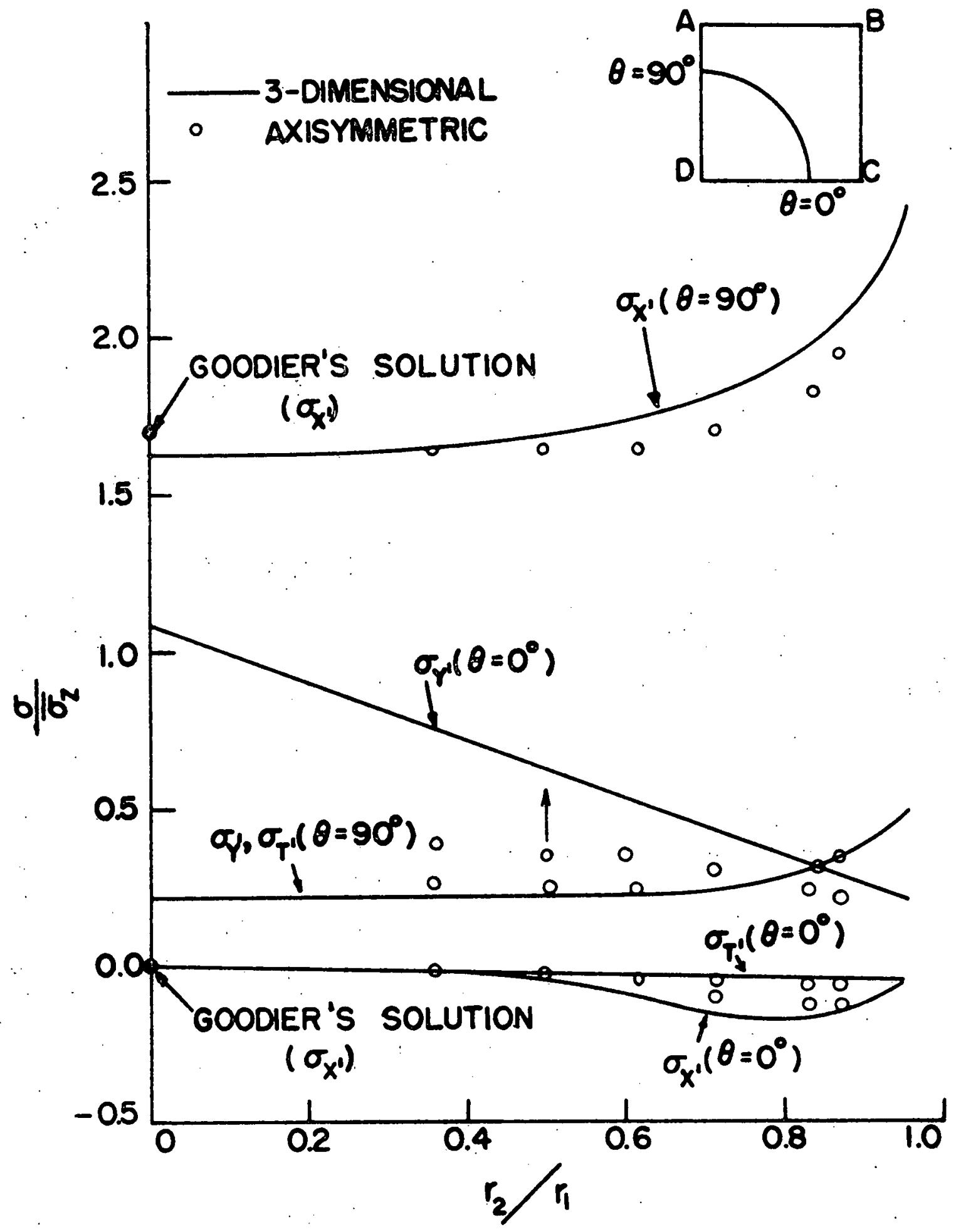

Fig. 35. Interfacial Stresses in Alumina-Filled Glass as a Function of $r_{2} / r_{1}$ 


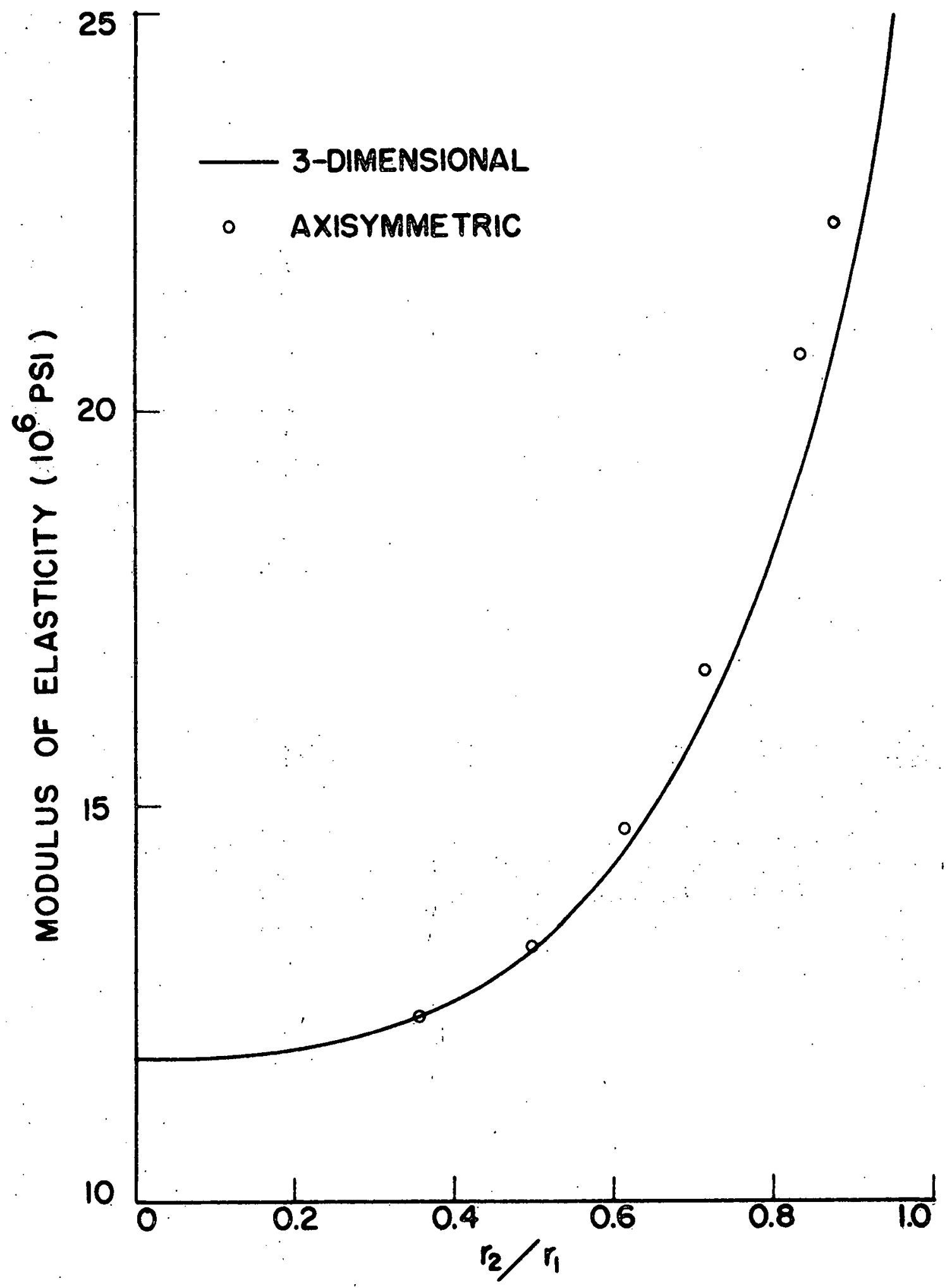

Fig. 36. Comparison of Modulus of Elasticity of Alumina Filled Glass as a Function of $r_{2} / r_{1}$. Predicted by ThreeDimensional and Axisymmetric Analyses 
Table 4. Predicted Modulus of Elasticity of Alumina Filled Glass

\begin{tabular}{cc}
\hline$r_{2} / r_{1}$ & $\begin{array}{c}\text { Modulus of Elasticity } \\
\left(10^{6} \mathrm{psi}\right)\end{array}$ \\
\hline 0.357 & 12.30 \\
0.714 & 16.06 \\
0.870 & 20.57 \\
0.952 & 24.96 \\
\hline
\end{tabular}

The predicted values of moduli of elasticity have been plotted as a function of volume fraction of $\mathrm{Al}_{2} \mathrm{O}_{3}$ in Fig. 37. For the three-dimensional analysis, the volume fractions have been calculated from cubic packing of the spheres as shown in Fig. 25. In the case of the axisymmetric analysis, the volume fractions can be calculated by assuming a sphere inside a cylinder, hexagonal packing or a cubic packing of the spheres as shown in Fig. 8 and Table 1. At lower values of the ratio of $r_{2} / r_{1}$ the volume fractions calculated by assuming three different packings do not differ appreciably but at $r_{2} / r_{1}=0.87$, the volume fraction may be assumed to be 34.43 or 43.83 depending upon the assumed packing. The values of modulus of elasticity predicted by the axisymmetric analysis have been plotted against the three volume fractions in Fig. 37. At lower volume fractions all the values compare satisfactorily with the three-dimensional stress analysis. However, at higher $r_{2} / r_{1}$ ratio the values plotted against volume fractions calculated by assuming a hexagonal packing agree very well with the results of the three-dimensional analysis, which lie in between the other two cases. 


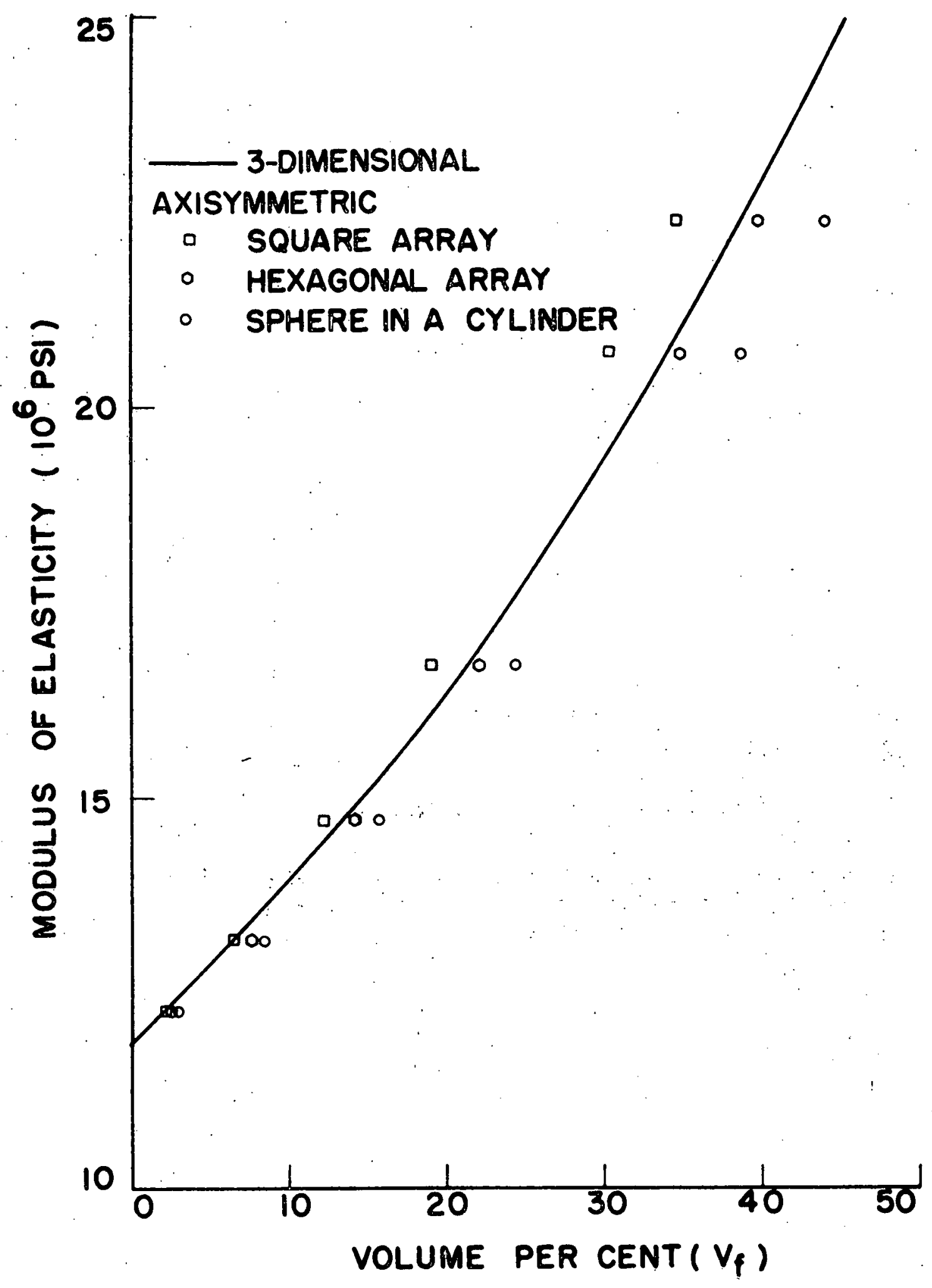

Fig. 37. Comparison of Modulus of Elasticity of Alumina-Filled Glass as a Function of Alumina Contents, Predicted by Three-Dimensional and Axisymmetric Analyses 
The present results strongly suggest that the interparticle spacing is a more significant parameter than volume fraction for presenting the internal stress distributions in a composite material. An average volume fraction of the filler is usually a more familiar term to the investigators. The interparticle spacing and the volume fraction can be easily related to each other in a composite with the uniform distribution of filler particles. However, the real composite always has some non-uniformity. Thus, in a region of the composite, where the concentration of the filler is higher than the average filler concentration, the internal stresses may be significantly higher than would be expected on the basis of a uniform distribution of filler porticles.

The results of the axisymmetric analysis and the three-dimensional analysis agree very well when presented as a function of interparticle spacing (which is uniquely determined by the ratio $\left.r_{2} / r_{1}\right)$. The axisymmetric analysis may be used without much loss of accuracy in place of a relatively complicated and much more costly three-dimensional analysis of composite materials. It may be mentioned here that the cost of obtaining information from the SAFE-3D ${ }^{(19)}$ program is about 20 times that of obtaining the same information from Wilson's ${ }^{(13)}$ program for the analysis of axisymmetric solids. 


\section{ELASTIC ANALYSIS OF THREE PHASE FIBROUS COMPOSITES}

Introduction

The many outstanding features of high performance fibrous composites have made them attractive structural materials. Oriented fiber reinforcement offers a strengthened and stiffened material having a high strength-to-weight ratio. Additional advantages of fibrous composites include improved behavior at high temperatures, the production of structural forms otherwise inconvenient or impossible and controlled anisotropy in physical properties.

In a unidirectional fiber reinforced composite the matrix serves two purposes, namely, to transfer the load to the fibers and to bind the fibers together. In the case of continuous fiber reinforcement, the effect of fiber ends, where the load is transferred by the matrix, is insignificant. The stress is assumed to be constant over the whole length of the fibers. The principal purpose of the matrix is to bind the fibers together. The strength of the composite is then dependent upon the strength of the fibers. However, in studying the fracture of continuous fiber composites it has been found that individual fibers fail well before the entire composite fractures. In this case load transferred to the broken fibers by the matrix and the interfacial conditions may thus influence composite fracture particularly as the number of broken fibers increases.

In the discontinuous fiber-Teinforced composite, the attainment of high strength in the composite will depend upon efficient load transfer from the matrix to the fibers. Therefore, it is of considerable interest to understand how stress builds up in each individual fiber. A study of the length required for effective reinforcement and the factors influencing this length such as the properties of the material at the interface and the fiber end condition, should thus be helpful in guiding the development of composites. 
It is well known that in discontinuous fiber reinforced systems with all fiber axes parallel to the direction of loading, the mechanism of load transfer from matrix to the fiber is an interfacial shear stress. A number of analytical studies concerning this shear stress transfer have been carried out using simplified models. Fiber-matrix interaction has been studied for elastic matrices by Cox ${ }^{(20)}$, Dow ${ }^{(21)}$, and Rosen ${ }^{(22)}$. They give expressions for axial fiber stress and for the shear stress at the fiber matrix interface as a function of position along the fiber length. These expressions are quite similar to each other, although different assumptions were made in deriving them. Tyson and Davies ${ }^{(23)}$, and Schuster and Scala ${ }^{(24)}$ measured interfacial shear stress between a metal fiber and epoxy resin by using photoelastic techniques. Studies of Fujiwara ${ }^{(25)}$ for resin-fiber load transfer in a single fiberresin composite indicate that the stress distribution depends upon glass fiber finishes, especially under wet conditions. Carrara and McGarry ${ }^{(26)}$ studied the effect of fiber end geometry on the stresses near the end of an elastic fiber embedded in an elastic matrix. They found that the stresses depend strongly on the geometry of the fiber tip. More recently, MocLaughlin and Barker ${ }^{(27)}$ investigated the effect of modulus ratio on stress near a discontinuous fiber. They analyzed a twodimensional plane stress composite configuration using moire strain analysis and finite element analysis.

One of the most important factors influencing the transfer of load from the matrix to the fibers is the interface condition. This factor has been thus far largely ignored by investigators. It was pointed out in Section II that a weak interface completely changes the stress distribution and results in low modulus of elasticity of the composite. A strong interface allows an efficient transfer of load to the fibers and would produce a composite with high tensile strength and high modulus of elasticity, therefore a low ultimate elongation. The low ultimate elongation indicates a lower energy absorption of the composite. Some intermediate properties 
of the interface may produce a composite which would have a higher impact strength without much loss of tensile strength. However, a very detailed study assigning different property values to the material at the interface is needed to understand the actual role of an interface on the load carrying capacity of the composite material. Such a study should be helpful both in predicting the behavior of a composite in which the interfacial conditions can be varied and in developing a composite to meet the requirements regarding the final property values. The present investigations were undertaken with this idea and interfaces with properties varying over a wide range were studied. This study, once again, was carried out using the axisymmetric analysis of composite materials with the computer program written by Wilson (13).

\section{Representative Areo}

Unidirectional discontinuous fibers were assumed to be packed in a regular array as shown in Fig. 38. Although this does not represent an actual packing of the fibers in the composite, this idealization is necessary for an axisymmetric analysis. The relation of the model to the actual composite will be discussed further in the next section.

It was assumed that the fibrous composite could be approximated by a cell (Fig. 39) which, when rotated $360^{\circ}$ around axis $A D$ produces a cylinder embedded within a cylinder. The interfiber spacing is equal to $2\left(r_{1}-r_{2}\right)$ in both directions as shown in Fig. 39. The finite elements used for the case $\frac{r_{2}}{r_{1}}=0.67$, are shown in Fig. 40(A). Based on a cylinder within a cylinder, this corresponds to a fiber volume fraction equal to 42.4 percent. The fiber aspect ratio used (ratio of fiber length to fiber diameter, $1 / d$ ) is 10.375 . The elements adjacent to the fiber (shaded elements in Fig. 40(B)) have been assumed to represent the finite thickness of the interface. The property values (mainly the modulus of elasticity and Poisson's 


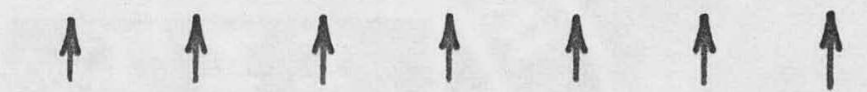
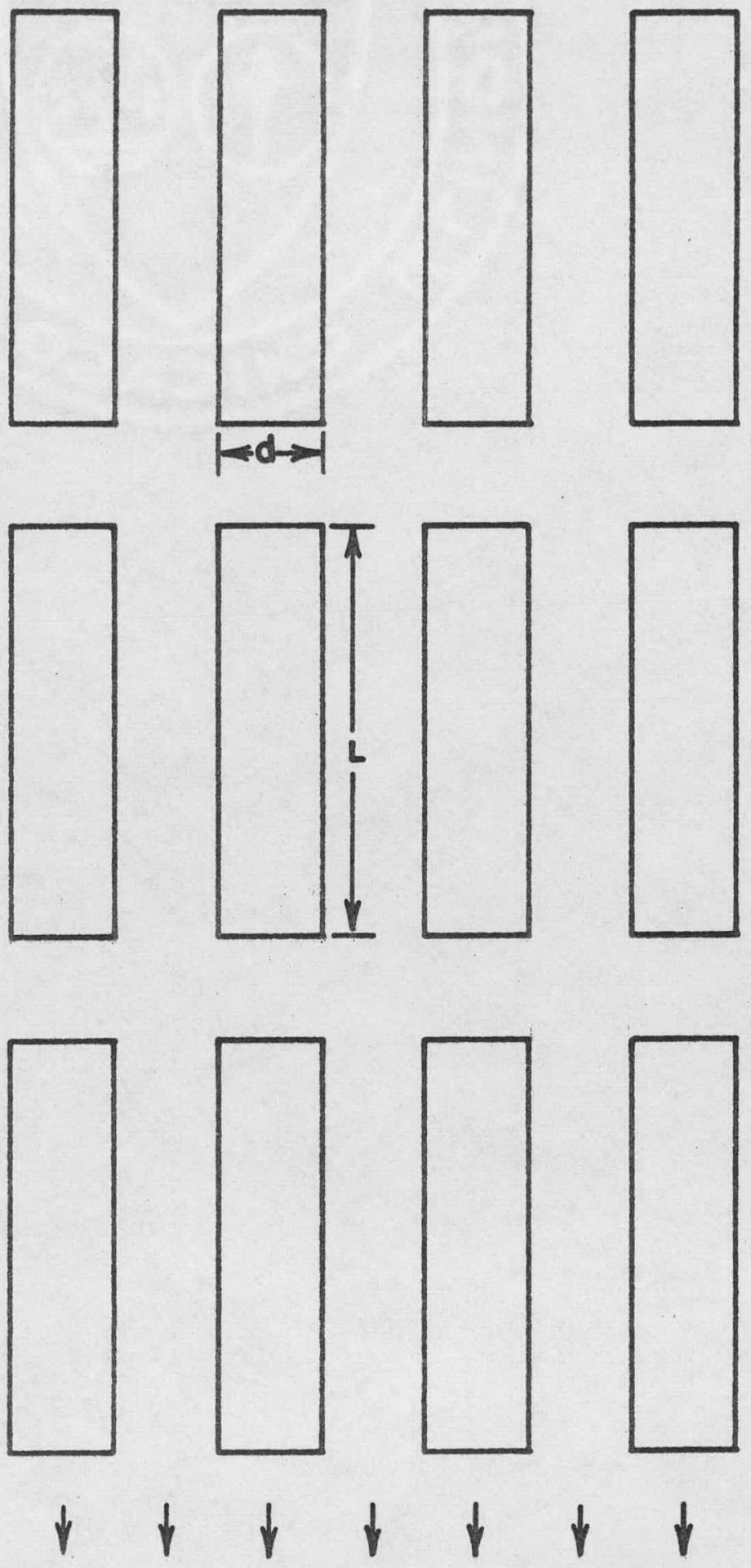

$\downarrow \quad \downarrow$

$\downarrow$

Fig. 38. Symmetrical Packing of Discontinuous Fibers in a Composite 


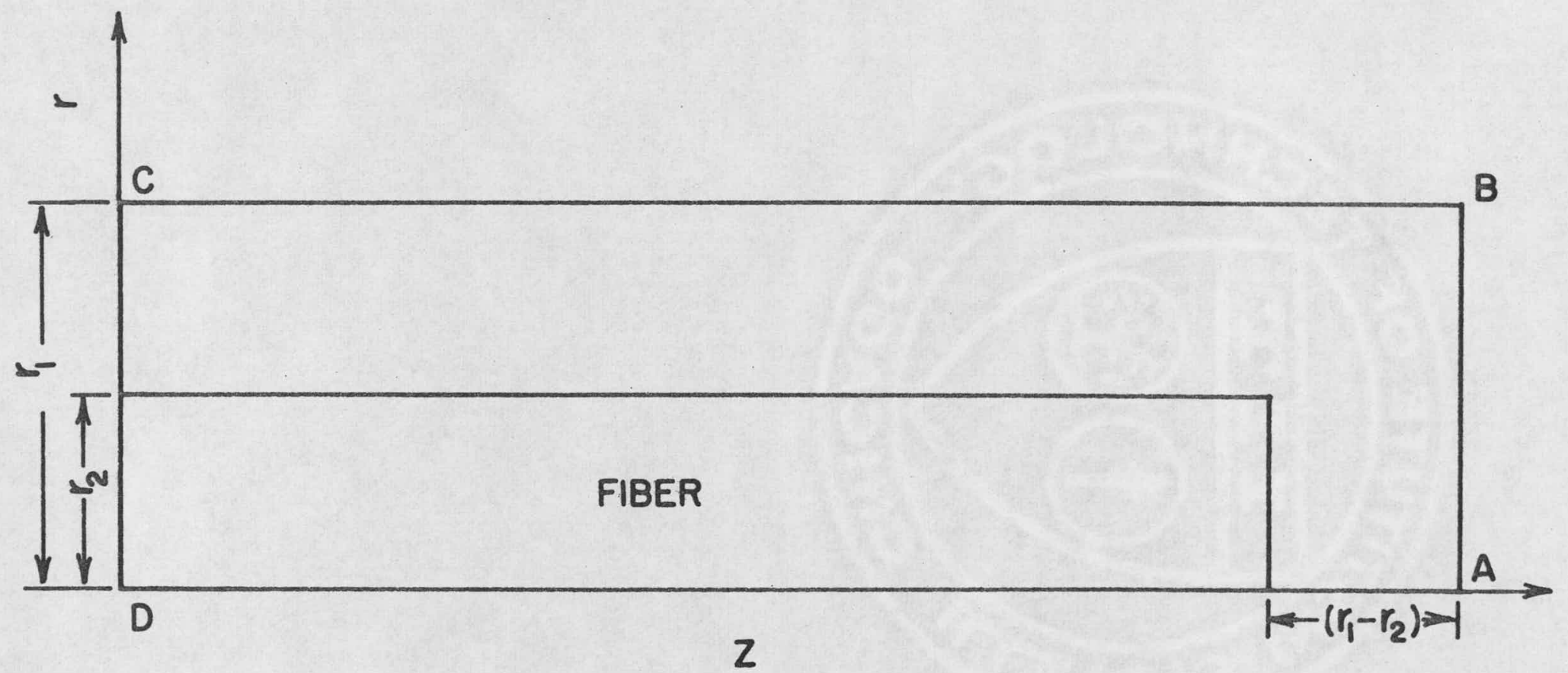

Fig. 39. Cell for Axisymmetric Representation of Fibrous Composite 

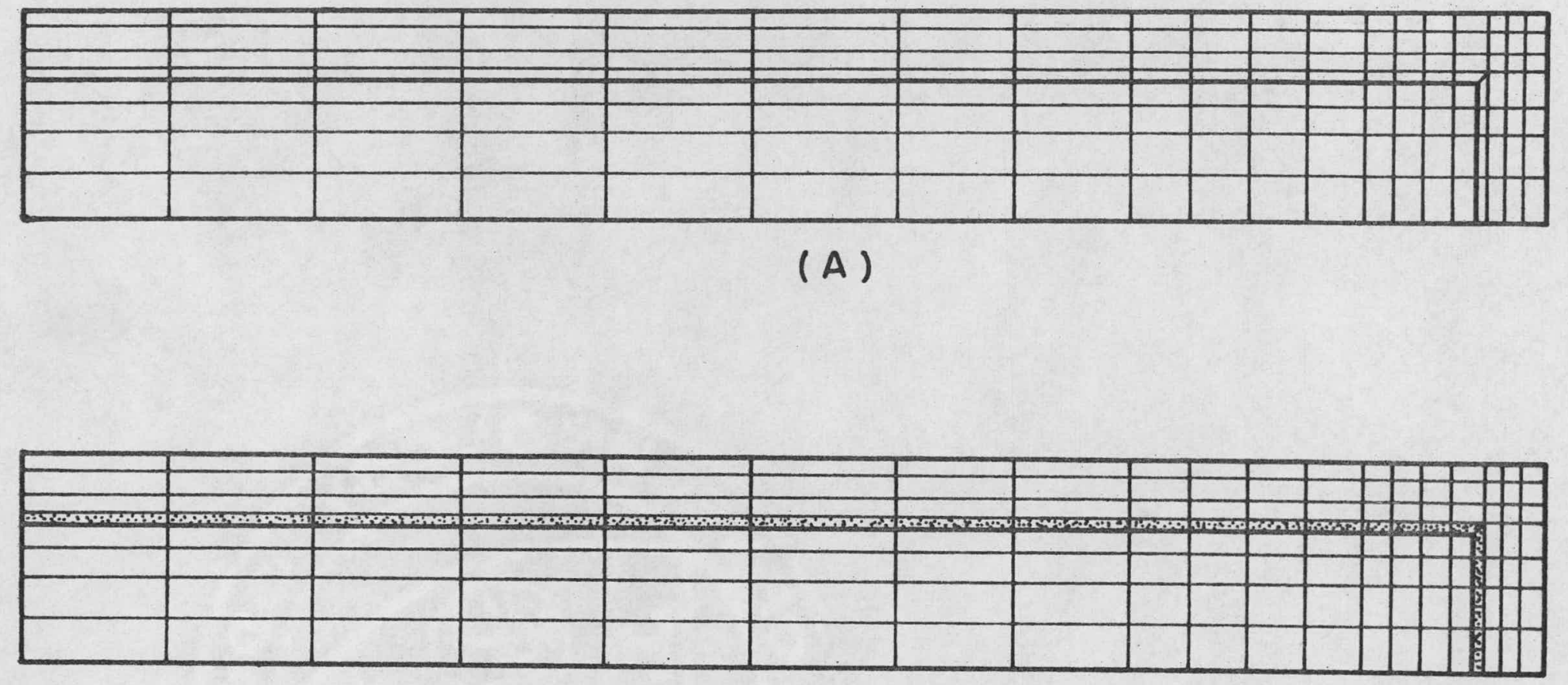

(B)

Fig. 40. (A) Finite Element Grid for $r_{2} / r_{1}=0.67$, and (B) Finite Thickness of the Interface
Represented by Shaded Elements 
ratio in the present case) assigned to these elements are changed to simulate a change in the interface conditions. A high modulus of elasticity of the interface represents a strong interface capable of transferring more load whereas a low modulus represents a weak interface. The shaded elements account for 7.76 percent of the total volume. In some cases the thickness of the interface was reduced by a half to study the effect of this change. The elements adjacent to the fiber end may be assigned property values different from those for the interface. This enables one to study the effect of fiber end condition on the stress distribution. For example, a very low modulus of elasticity for these elements may be assumed to represent a debonded end because a negligible load will be transferred through the fiber end in this case.

\section{Boundary Conditions and Component Properties.}

Stresses in three dimensions were calculated in all the elements shown in Fig. 40 for various interface conditions. As in the case of the particulate composites, the stress-strain relations of the matrix and the fibers were assumed elastic. The stress-strain relations for the materials at the interface and the fiber end were also assumed elastic. It was also assumed that the composite is loaded by a force in the $z$ direction and that no tractions are applied in the $r$ direction. These assumptions lead to the boundary conditions identical to those for the particulate composite described in Section II. Therefore, the procedure to satisfy these boundary conditions and the subsequent calculation of the composite modulus and Poisson's ratio was also the same as described in the Appendix.

The following component properties were assumed:

\begin{tabular}{|c|c|}
\hline Matrix & $\begin{array}{l}E=0.4 \times 10^{6} \text { psi } \\
\nu=0.35\end{array}$ \\
\hline Fibers & $\begin{array}{l}E=11.8 \times 10^{6} \text { psi } \\
\nu=0.197\end{array}$ \\
\hline
\end{tabular}


These properties represent a typical glass fiber reinforced polymeric material. Properties of the interface were varied over a wide range. Investigations were carried out using eleven different combinations of property values as shown in Table 5.

Table 5. Properties of the Interface

\begin{tabular}{ccccc}
\hline S. No. & $E$ (psi) & $\nu$ & Vol. Percent & E ot the fiber end \\
\hline 1 & $8 \times 10^{6}$ & 0.2 & 7.76 & $8 \times 10^{6}$ \\
2 & $8 \times 10^{6}$ & 0.35 & 7.76 & $8 \times 10^{6}$ \\
3 & $8 \times 10^{6}$ & 0.45 & 7.76 & $8 \times 10^{6}$ \\
4 & $8 \times 10^{6}$ & 0.2 & 3.79 & 100 \\
5 & $8 \times 10^{6}$ & 0.2 & 7.76 & 100 \\
6 & $0.8 \times 10^{6}$ & 0.2 & 7.76 & $0.8 \times 10^{6}$ \\
7 & $0.4 \times 10^{6}$ & 0.2 & 3.79 & $0.4 \times 10^{6}$ \\
8 & $0.1 \times 10^{6}$ & 0.2 & 3.79 & $0.1 \times 10^{6}$ \\
9 & 10,000 & 0.2 & 3.79 & 10,000 \\
10 & 1,000 & 0.2 & 7.76 & 1,000 \\
11 & 100 & 0.2 & 7.76 & 100 \\
\hline
\end{tabular}

Modulus of elasticity of the interface has been varied from a very high value of $8 \times 10^{6}$ psi which is close to that of the fibers to a very low value of only 100 psi which may be considered to represent debonding of the fibers from the matrix. The first three sets of properties have been selected to study the effect of varying Poisson's ratio of the interface. Modulus of elasticity of the elements adjacent to the fiber end is the same as that of the interface for all cases except for 4 and 5 . For these two cases it has a very low value (100 psi) compared to that of the interface. This represents a case of strong interface with debonded fiber end. The 
only difference in the case 4 and the case 5 is the thickness of the interfoce which would change the volume percent of the interface.

\section{Stresses}

The stresses in a two-phase fibrous composite (i.e., with the elements at the interface having the same properties as the matrix) have been shown in Figs. 4144. The stresses have been normalized with respect to the applied stress on the composite $\left(\sigma_{c}\right)$. In the figures $Z$ is the distance from the fiber end and $d$ is the fiber diameter. Variation of the axial stress in the fiber and the interfacial shear stress along the fiber is shown in Fig. 41. The fiber axial stress attains its maximum value in less than two fiber diameters from the fiber end. In about the same distance the interfacial shear stress drops down to zero. The axial stress at the fiber end is about $1.5 \sigma_{c}$ which indicates that the load transfer from the fiber end is significant. This can also be seen from Fig. 42 in which the variation in the radial direction of the matrix axial stress (in the elements adjacent to the fiber end) has been shown. The axial stress in the elements with $r / r_{2}$ less than one (where $r_{2}$ is the radius of the fiber) is nearly $1.3 \sigma_{c}$. This significant load transfer from the fiber end leads to a small ineffective fiber length over which the fiber does not carry the maximum stress.

The maximum fiber stress $\left(\sigma_{f}\right)$ in case of unidirectional long fibers can be calculated $(28)$ by assuming sharing of load between the fiber and the matrix as follows:

$$
\sigma_{c} A_{c}=\sigma_{f} A_{f}+\sigma_{m} A_{m}
$$

where $A$ represents the area. Since there is perfect bonding between the fiber and the matrix, the strain experienced by the composite is equal to the fiber strain and also the matrix strain

$$
\varepsilon_{c}=\epsilon_{m}=\varepsilon_{f}
$$




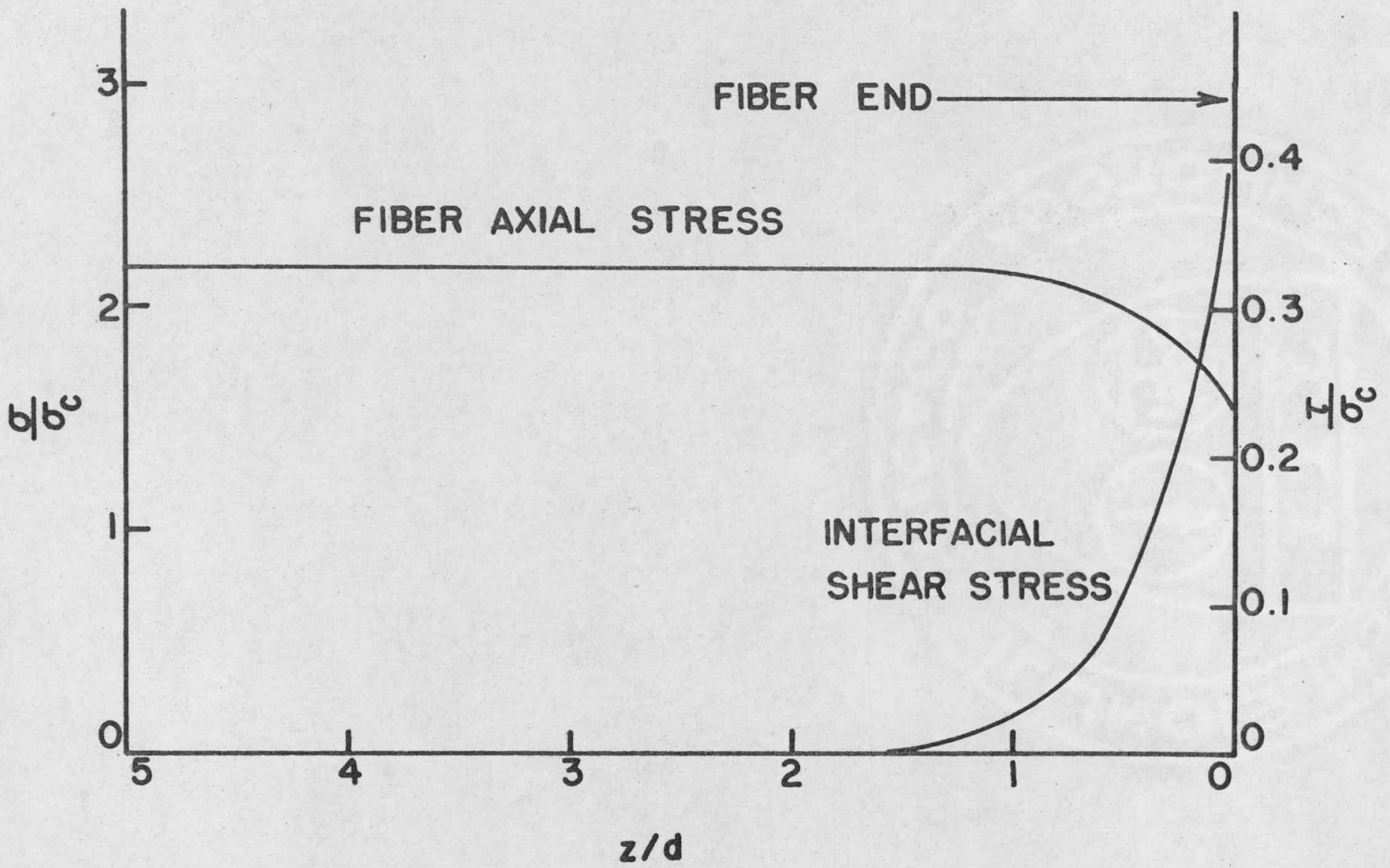

Fig. 41. Normalized Fiber Axial Stresses and Interfacial Shear Stresses Along Fiber Axis in a Two-Phase Composite 


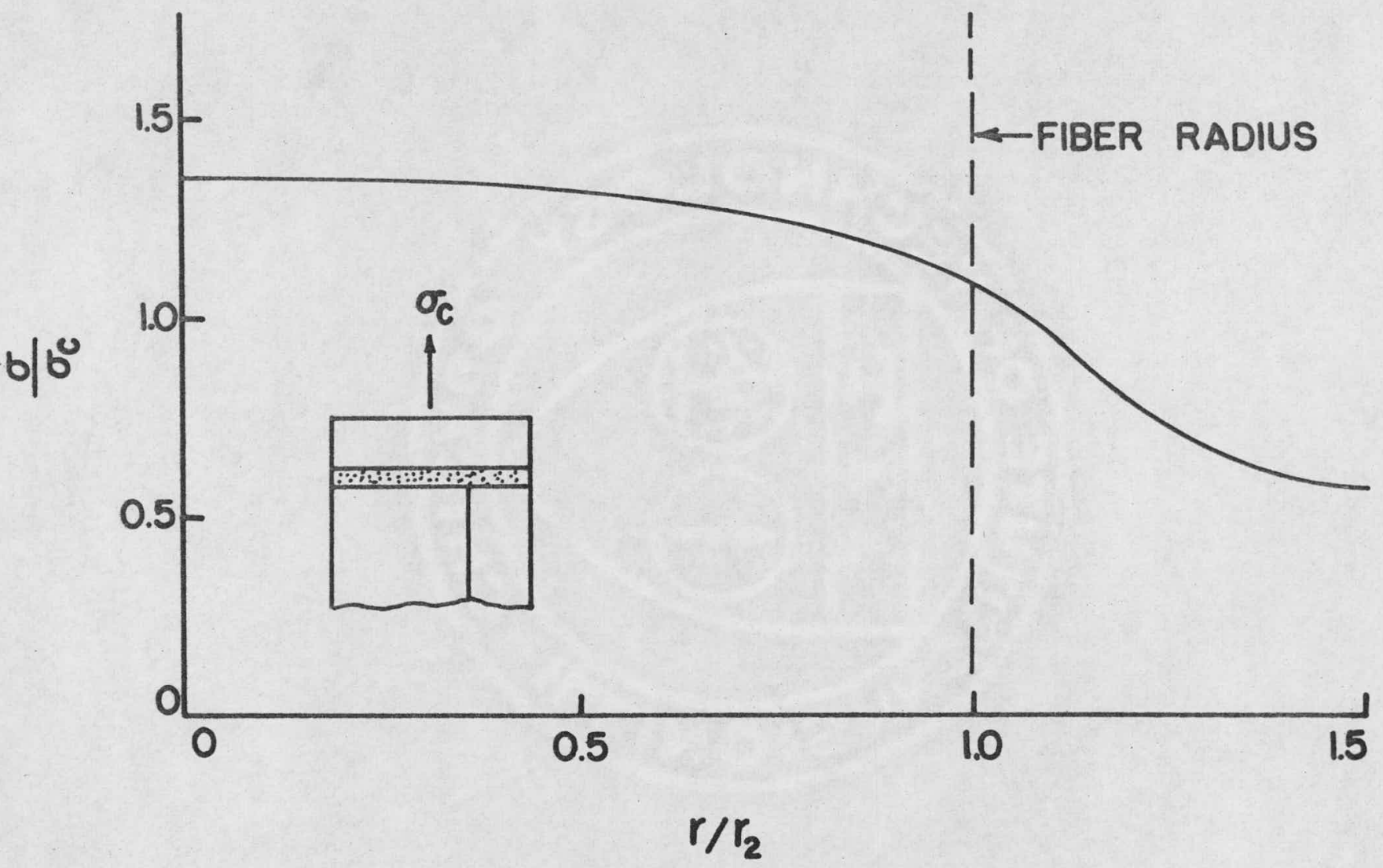

Fig. 42. Radial Variation of Normalized Axial Stresses in the Matrix of a Two-Phase Composite 


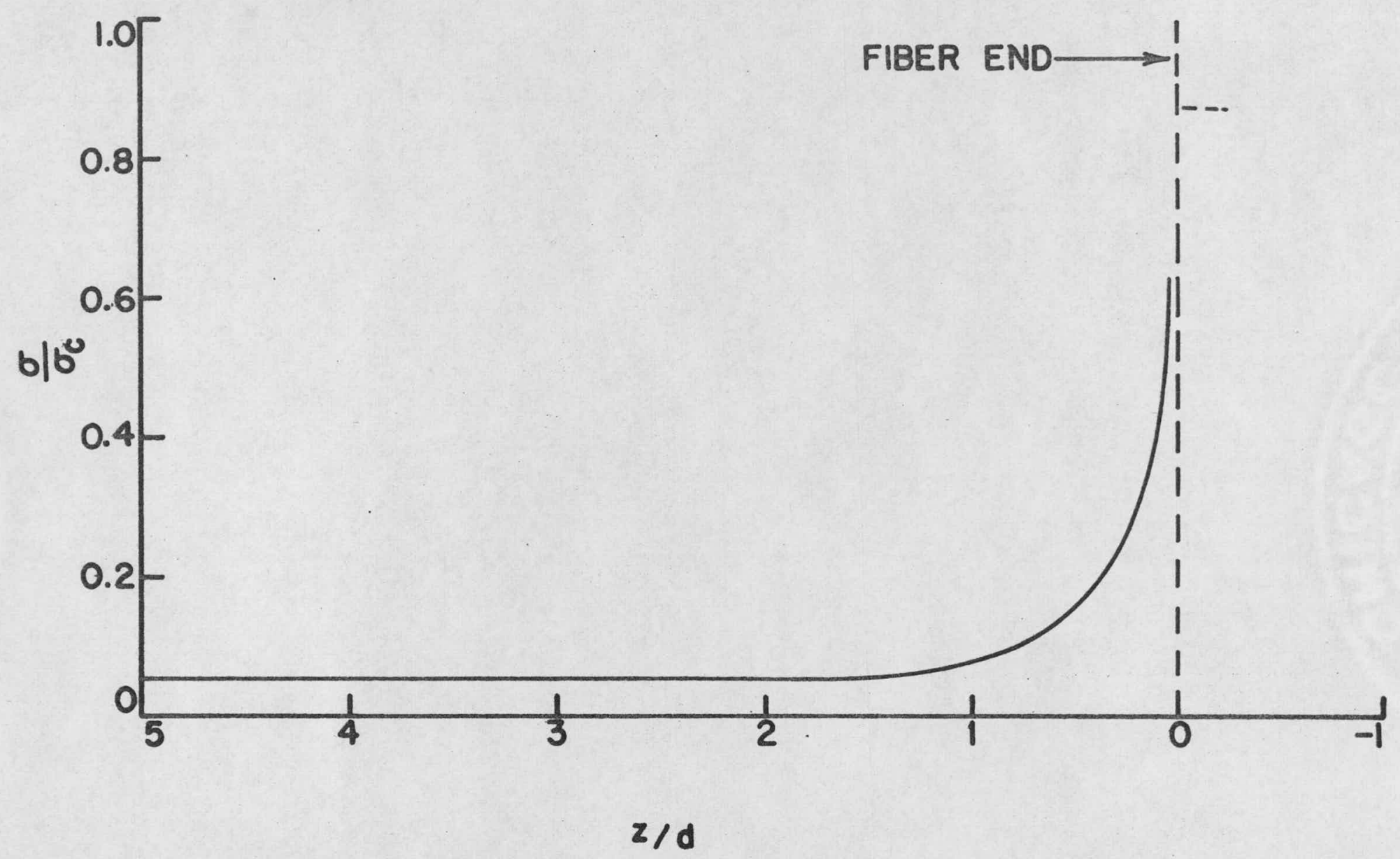

Fig. 43. Normalized Matrix Axial Stresses Along Fiber Axis in a Two-Phase Composite 


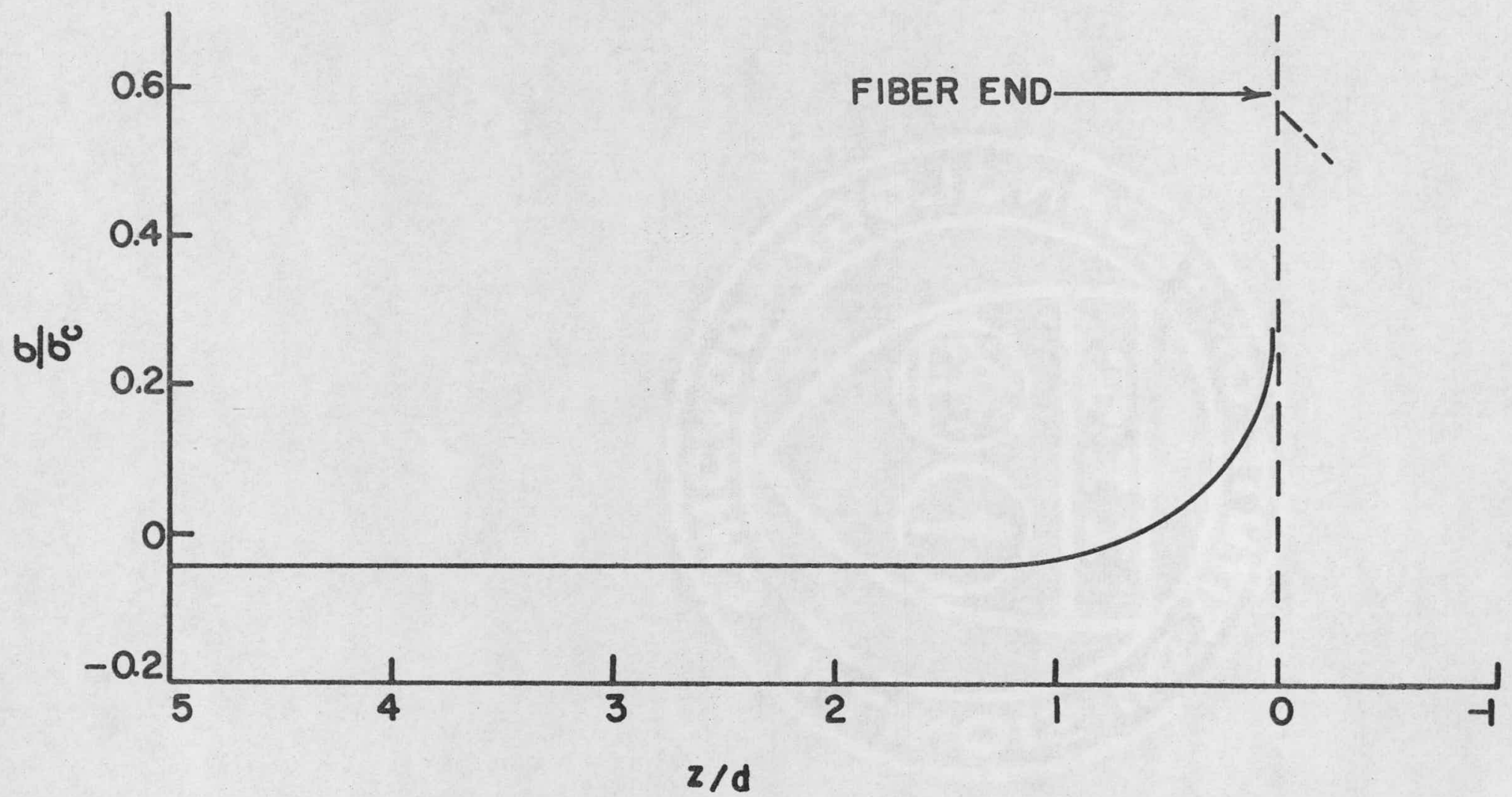

Fig. 44. Normalized Matrix Radial Stresses Along Fiber Axis in a Two-Phase Composite 
Therefore $\frac{\sigma_{m}}{E_{m}}=\frac{\sigma_{f}}{E_{f}}$

Combining equations ( 1 ) and (3)

$$
\frac{\sigma_{f}}{\sigma_{c}}=\frac{1}{\left(\frac{A_{f}}{A_{c}}+\frac{A_{m}}{A_{c}} \frac{E_{m}}{E_{f}}\right)}
$$

For the present case $\frac{A_{f}}{A_{c}}=\frac{4}{9}, \frac{A_{m}}{A_{c}}=\frac{5}{9}$ and $\frac{E_{m}}{E_{f}}=\frac{0.4}{\pi .8}$, thus

$$
\frac{\sigma_{f}}{\sigma_{c}}=2.15
$$

This ratio of fiber stress to the composite stress is very close to the one obtained by finite element analysis $\left(\frac{\sigma_{f}}{\sigma_{c}}=2.17\right)$ and shown in Fig. 41 .

Variation of the matrix axial stress and the matrix radial stress at the interface along the fiber length are shown in Figs. 43 and 44. In both cases the stresses increase sharply near the fiber end. Figs. 43 and 44 also show discontinuities in the stresses upon passing the fiber end. This is not unexpected because of the discontinuity in the material properties at this point.

\section{Effect of Interface}

The stress distributions for three phase composites were obtained for all the cases indicated in Table 5. In the first three cases, the Poisson's ratio of the interface was assigned three different values of $0.2,0.35$ and 0.45 while keeping all other properties unchanged. The stresses thus obtained in the three cases were almost identical to each other and hence they have not been plotted separately. These stresses have been shown along with the other stress distributions for different interface elastic moduli. 
Distributions of fiber axial stress along the length are shown in Fig. 45 for interface moduli varying from $8 \times 10^{6}$ psi to 100 psi. When the interface modulus is near the matrix modulus or higher, the fiber axial stress attains a maximum value within two fiber diameters from the fiber end. The stress distributions for the three case of interface moduli $\left(E=0.1 \times 10^{6}, 0.8 \times 10^{6}\right.$ and $\left.8 \times 10^{6}\right)$ are very similar to each other.: As the interface modulus decreases to 10,000 psi, the fiber axial stress attaịns its maximum value in about four fiber diameter from the fiber end. But as the interface modulus further decreases to 1,000 psi or 100 psi, the fiber axial stress does not reach a constant value with the present fiber length of ten fiber diameters. Due to the low interface modulus, the interface does not transfer load from matrix to the fiber efficiently. In fact the modulus of 100 psi is so low that it represents the case of complete debonding of the fiber from the matrix as will be shown later.

Interfacial shear stress distributions for all the above cases, except for interface modulus of $0.8 \times 10^{6}$ psi, have been shown in Fig. 46. The stress distribution for the interface modulus of $0.8 \times 10^{6}$ psi is very close to the one for the interface modulus of $0.1 \times 10^{6}$ psi. The stress distributions in Fig. 45 are related to those in Fig. 46 because fiber stress build up is related to the shear stress at the interface. The relation between the fiber axial stress and the interfacial shear stress can be obtained by considering equilibrium of an infinitesimal fiber length dz (Fig. 47) as follows:

$$
\begin{aligned}
& \left(\sigma_{f}+d \sigma_{f}\right) \pi r^{2}=\sigma_{f} \pi r^{2}+\tau 2 \pi r d Z \\
& \therefore d \sigma_{f}=\frac{2}{r} T d Z
\end{aligned}
$$

Integrating (6),

$$
\sigma_{f}=\sigma_{0}+\frac{2}{r} \int_{0}^{Z} \tau d Z
$$




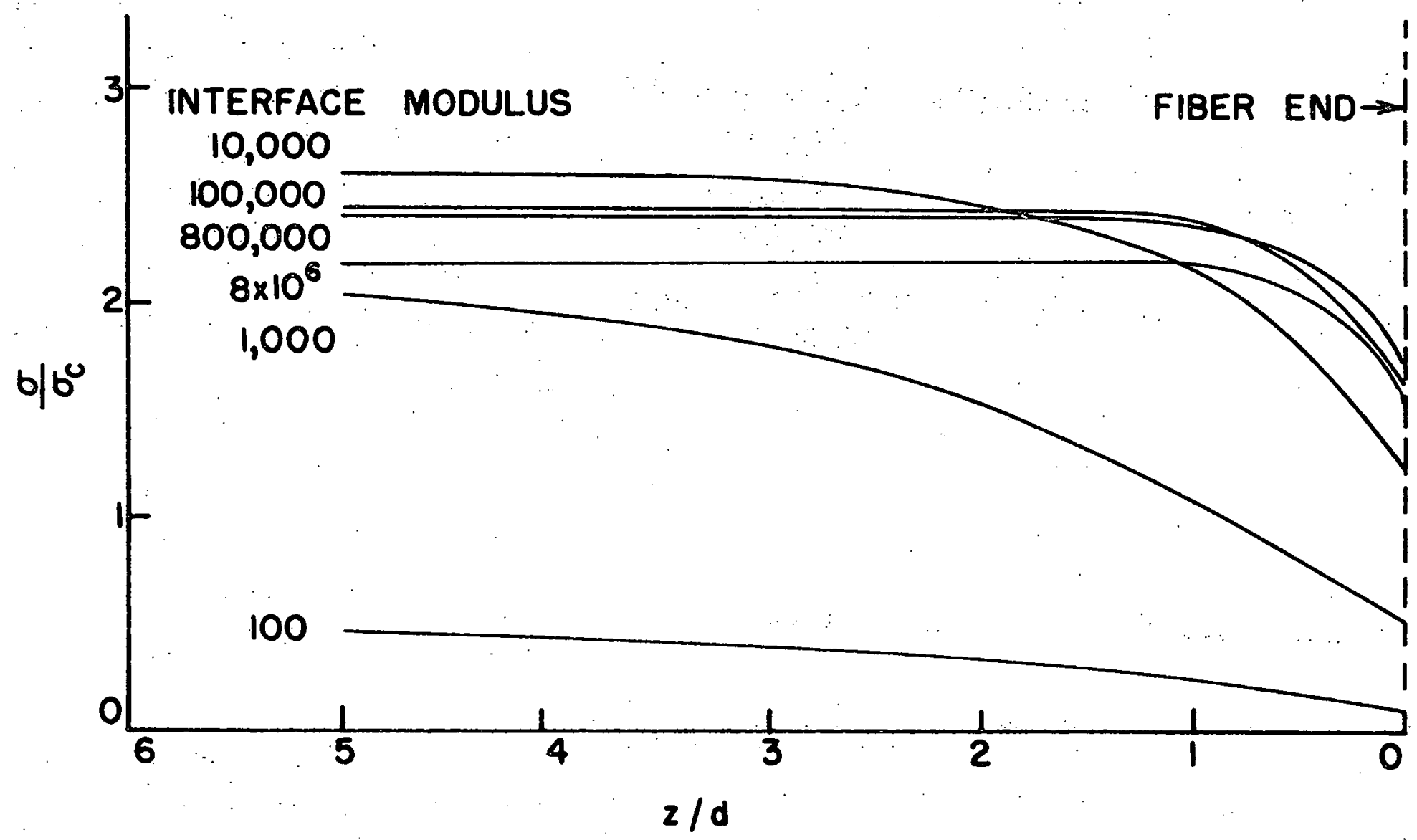

Fig. 45. Normalized Fiber Axial Stresses Along Fiber Axis in a Three-Phase Composite 


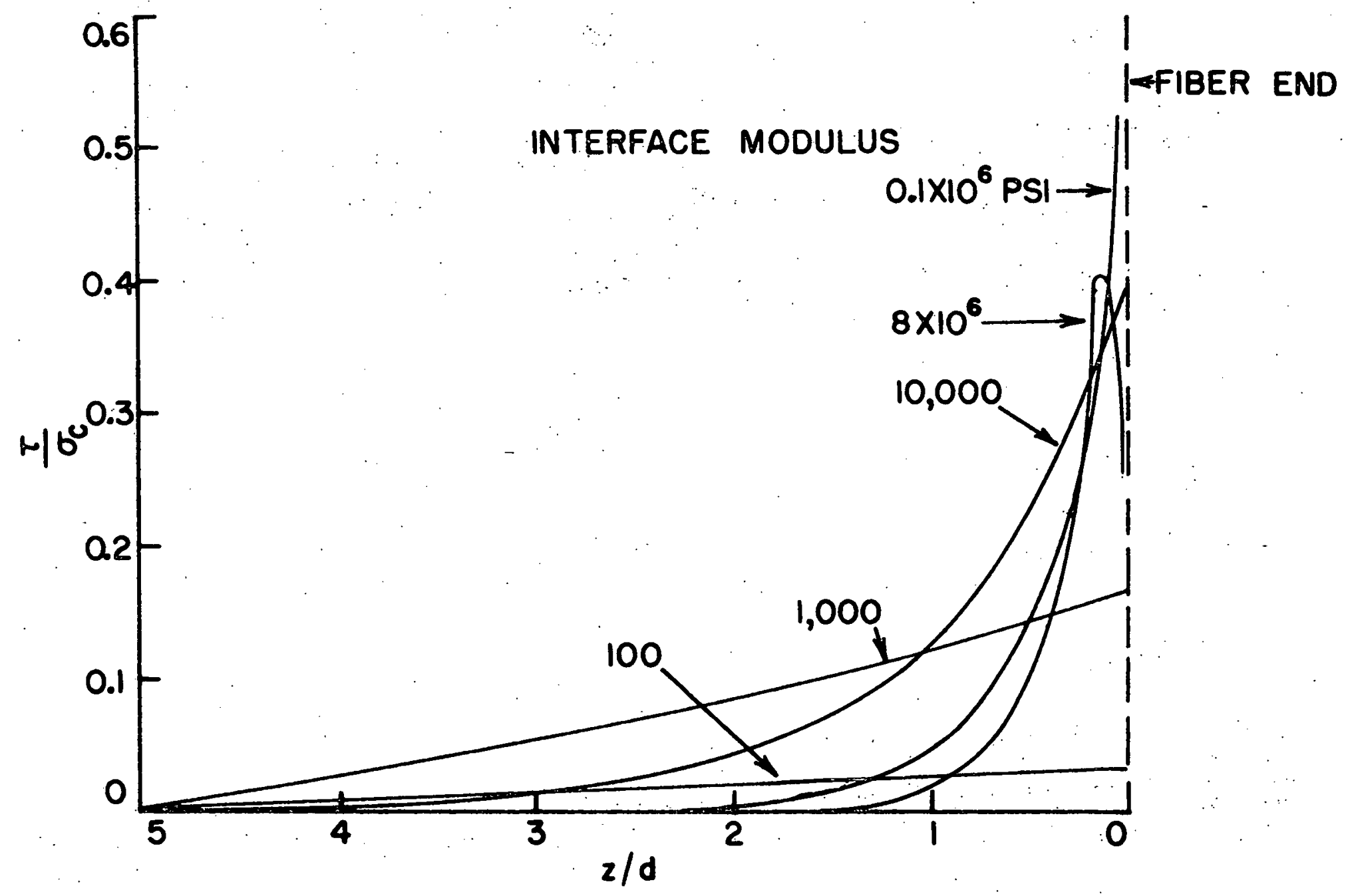

Fig. 46. Normalized Interfacial Shear Stresses. Along Fiber Axis in a Three-Phase Composite 


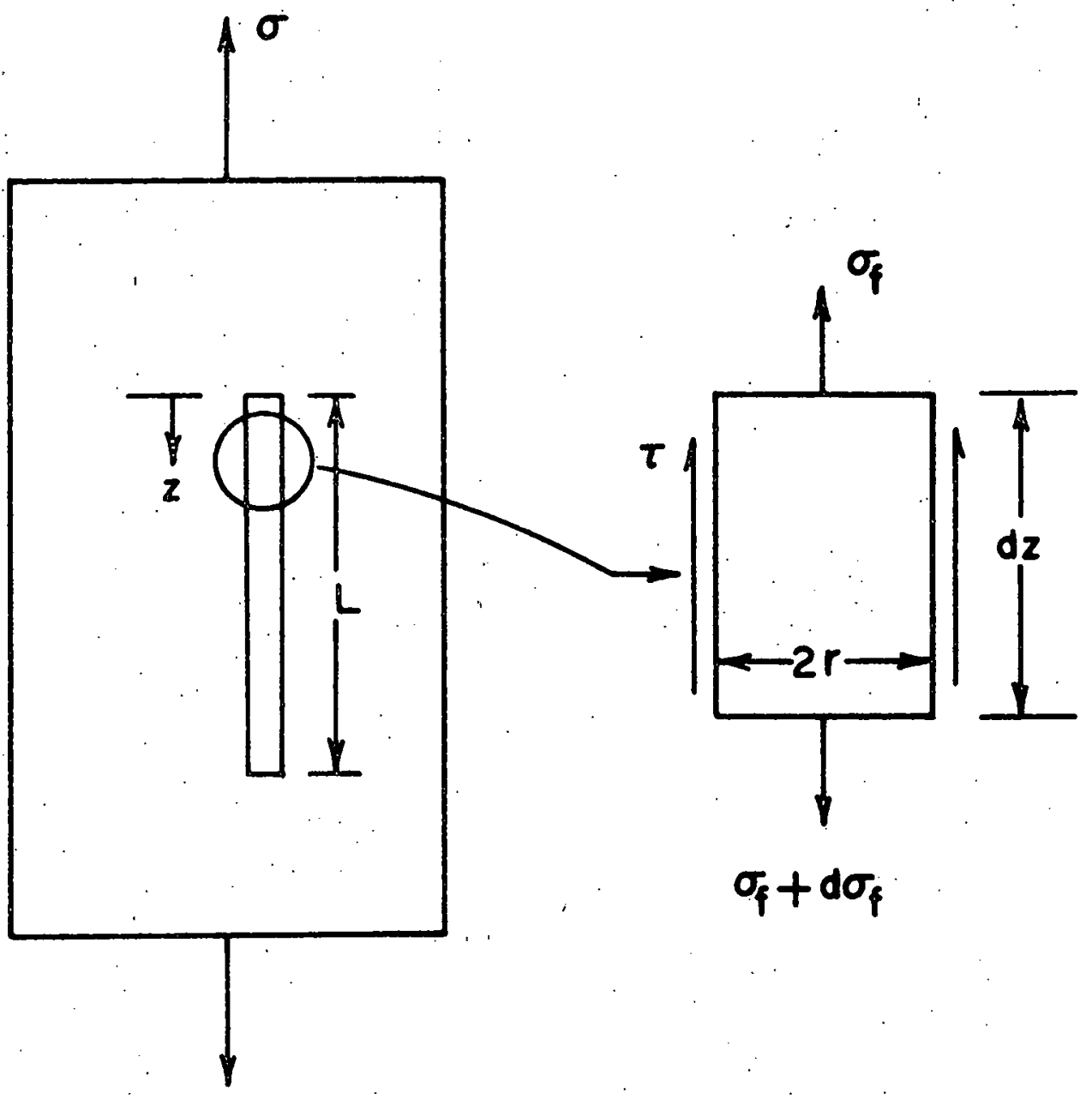

Fig. 47. Equilibrium of Infinitesimal Fiber Length 
where $\sigma_{0}$ is the stress at the fiber end and the exact form of $\tau$ will depend upon relative properties of the fiber, interface and the matrix. Equation (7) shows that the area under the plot of shear stress versus the length of the fiber represents the change in fiber stress or in other words (from Eq. 6) the slope of curve for fiber stress is given by the magnitude of shear stress at the interface at that point. It can be seen that the stress distributions in Fig. 45 are consistent with those in Fig. 46. The shear stresses at the fiber end for interface moduli of $0.1 \times 10^{6}$ and $8 \times 10^{6} \mathrm{psi}$ are high and they drop down to zero in about two fiber diameters. Due to the high interfacial shear stress, the fiber axial stress increases rapidly and reaches a constant value as the shear stress drops to zero. For the interface modulus of 10,000 psi the shear stress does not drop to zero as quickly as in the previous cases and thus leads to a higher maximum stress in the fiber. Normalized shear stress at the fiber end for interface modulus of 1000 psi is less than half of those in the previous cases. However, this does not decrease very fast away from the fiber end and hence the axial stress in the fiber builds up to about twice the stress on the composite. It may be expected that in this case the fiber stress could reach a constant value if the fiber was long enough. Interfacial shear stress in the case of interface modulus of 100 psi is very small and therefore very little load transfer is possible from the matrix to the fiber.

Fiber axial stress at the end has been plotted as function of log of interface modulus in Fig. 48. This stress gives an idea of the load transfer through the fiber end. At very low interface modulus the stress at the fiber end is very small indicating that no significant load transfer takes place through the fiber end. The stress at the fiber end increases with the increase of interface modulus. When the interface modulus is close to or higher than the matrix modulus, the fiber end stress is quite high indicating substantial load transfer through the end. As the inferface modulus varies from $10^{5}$ psi to $8 \times 10^{6}$ psi there is not much change in the fiber end stress. 


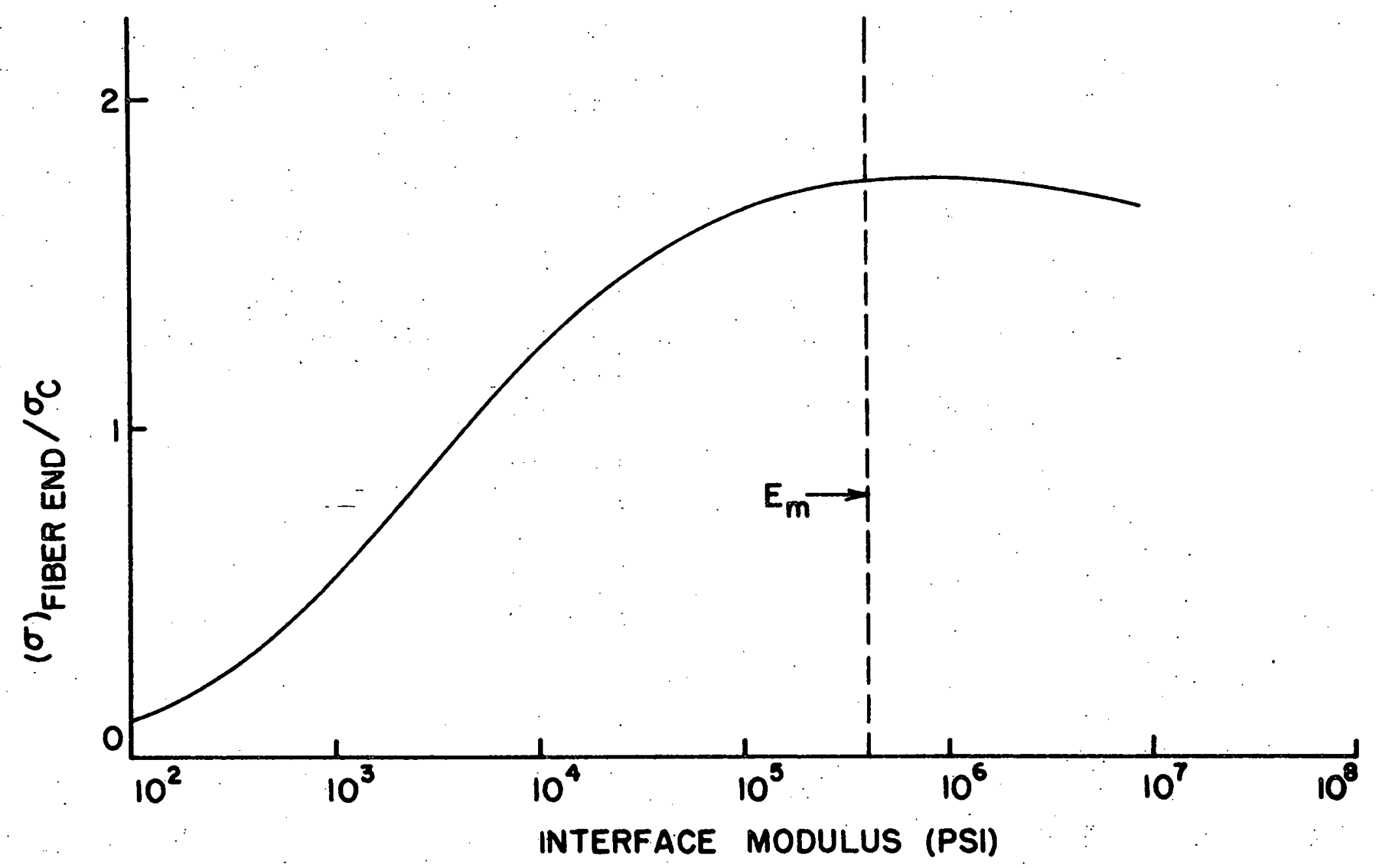

Fig. 48. Normalized Axial Stress at the Fiber End as a Function of Interface Modulus 
Variation in the radial direction of the matrix axial stress (in the elements adjacent to the fiber end, refer Fig. 42) has been shown in Fig. 49 for interface moduli of 100, 1,000,10,000 and 100,000 psi. For interface moduli higher than $10^{5}$ psi, the variations are almost identical to the one for modulus of $10^{5}$ psi. All the curves in Fig. 49 show a discontinuity at $\frac{r}{r_{2}}=1.0$ because of the discontinuity in the material properties at this point. In the case of interface modulus of $10^{5} \mathrm{psi}$, the matrix axial stress is about $1.5 \sigma_{c}$ for $r / r_{2}$ less than one and it sharply drops away from the fiber end. This is because the fiber carries more load than the matrix. For the interface modulus of 10,000 psi, the matrix axial stress does not change appreciably as $r / r_{2}$ varies from zero to 1.5 . In the cases of interface moduli of 100 and 1,000 psi, the matrix axial stress for $r / r_{2}$ less than one is very small but increases very sharply away from the fiber end. In these two cases the load transfer from the matrix to the fiber is very small because of the weak interface. Therefore most of the load is carried by the matrix.

Distribution of matrix axial stresses along the fiber length are shown in Fig. 50 for the interface moduli of 100,1,000,10,000 and $10^{5}$ psi. In all the cases the stresses increase sharply near the fiber end. They also show discontinuities in the stresses upon passing the fiber end because of the physical discontinuity at this point. In the cases of interface moduli of $10^{4}$ and $10^{5}$ psi, the stresses away from the fiber end drop to a very low value indicating that most of the load has been transferred to the fibers. However, low modulus of the interface (100 or 1,000 psi) does not help in the transfer of load from matrix to the 'fibers and therefore the axial stresses in the matrix away from the fiber end are considerably higher than the applied composite stress in these cases.

Radial stresses in the matrix have been plotted along the fiber length in Fig. 5! for interface moduli of 100, 1,000 and 10,000 psi. The stresses increase sharply near the fiber end. The stresses away from the fiber end are compressive 


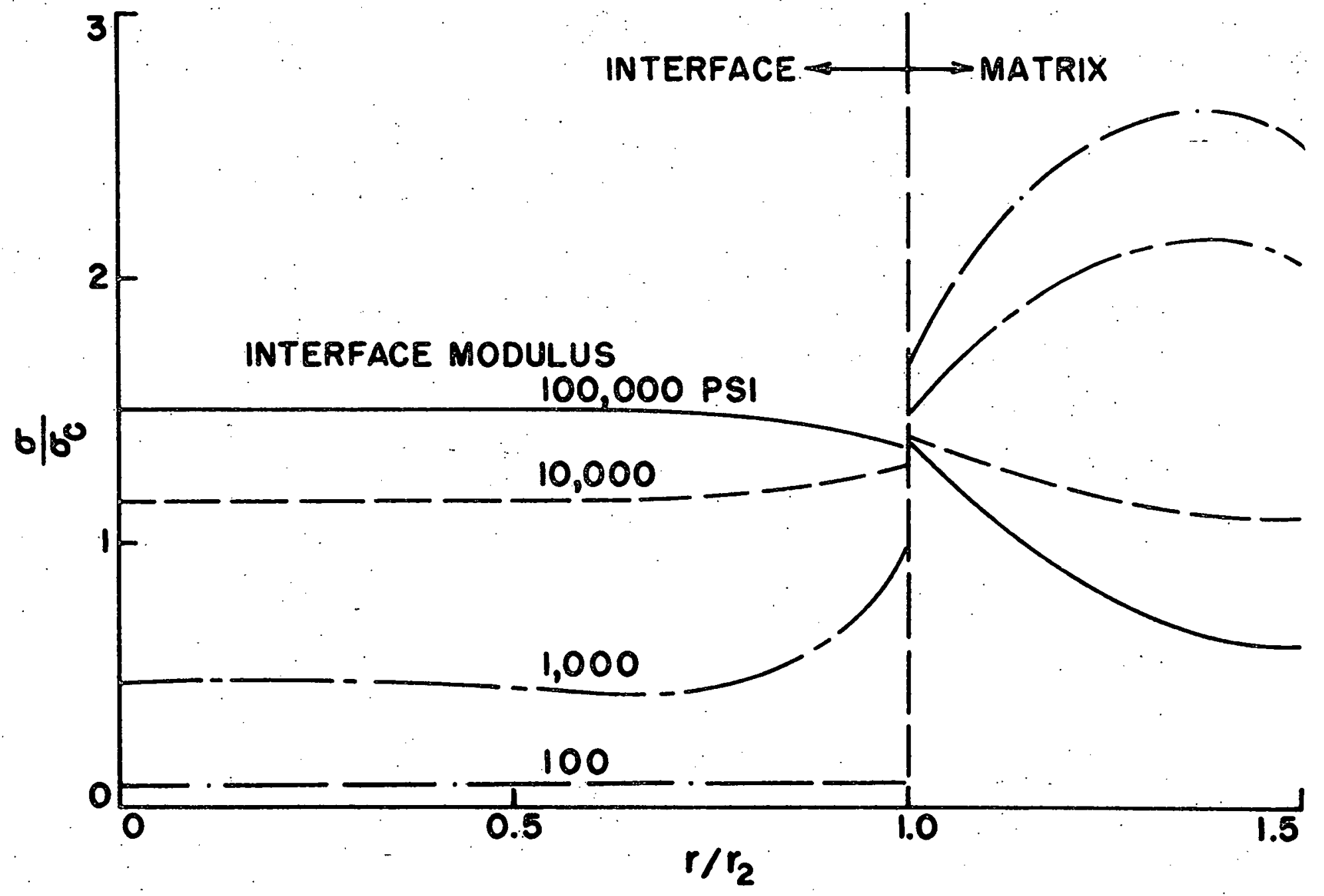

Fig. 49. Radial Variation of Axial Stresses Near Fiber End in a Three-Phase Composite 


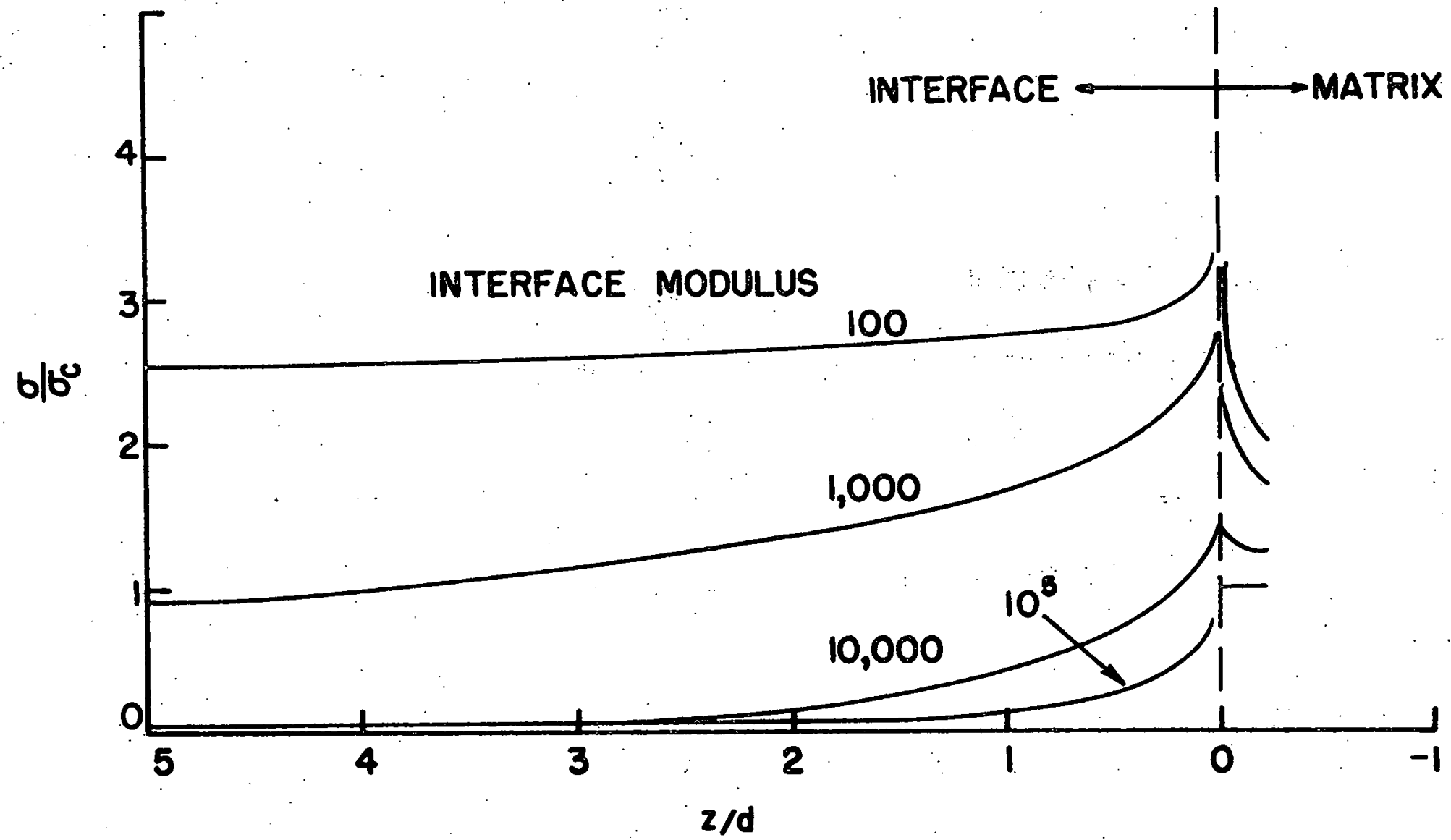

Fig. 50. Normalized Axial Stresses Along Fiber Axis in. Three-Phase Composite 


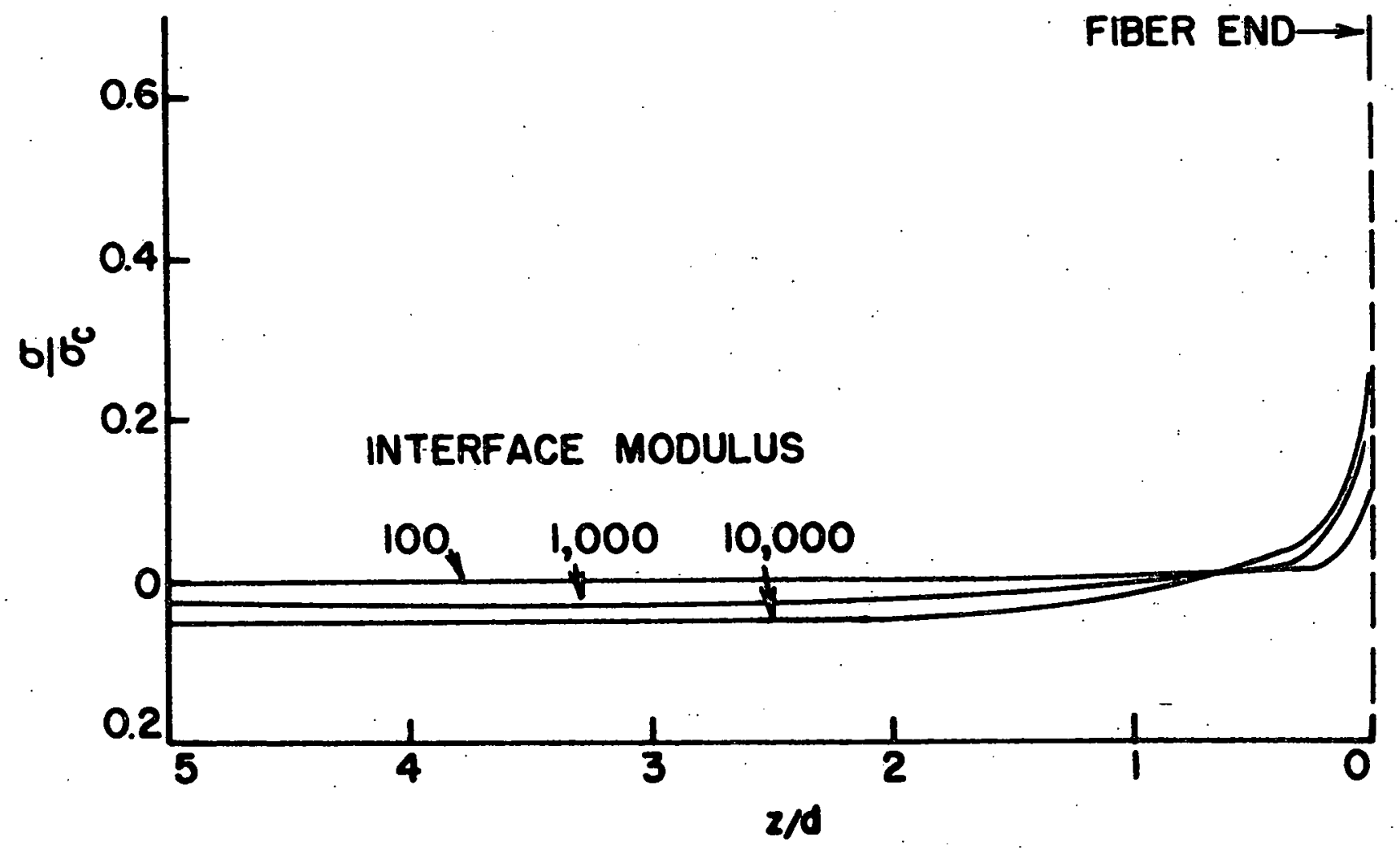

Fig. 51. Nomalized Matrix Radial Stress Along fiber Axis in a Three-Phase Composite 
in case of the interface moduli of 1,000 and 10,000 psi. For the interface modulus of 100 psi, the radial stresses in the matrix are zero. It was shown in Fig. 49 that the axial stresses in the matrix adjacent to the fiber end are nearly zero for interface modulus of 100 psi. Small magnitude of the stresses may be attributed to the size of the finite elements. Thus the normal stresses in the matrix adjacent to the fiber are zero indicating a free boundary. Therefore this represents a case of complete debonding of fibers from the matrix. This effect is the same as obtained in the case of particulate composites (Section II).

Composite modulus has been plotted as a function of log interface modulus in Fig. 52. At very low interface modulus the fibers do not contribute to the stiffness of the composite. As explained earlier, this is due to the fact that the weak interface does not permit any load transfer from the matrix to the fiber. Therefore, the composite behaves as if these were voids of the size of the fiber and the interface. As the interface modulus increases, the load transfer takes place from the matrix to the fiber and consequently the composite modulus increases as shown in Fig. 52. The Halpin and Tsai equation ${ }^{(29)}$ may also be used to calculate the modulus of the two phase composite with discontinuous fiber reinforcement. For the modulus in the longitudinal direction, the equation can be written as

$$
\frac{E_{L}}{E_{m}}=\frac{1+\frac{2 \ell}{d} \cdot V_{f} \cdot \eta_{L}}{-V_{f} \cdot \eta_{L}}
$$

where

$$
\eta_{L}=\frac{\left(E_{p} / E_{m}\right)-1}{\left(E_{p} / E_{m}\right)+2 d d}
$$

and $E_{m}, E_{f}$ and $E_{L}$ are the matrix, fiber and the composite moduli respectively. For the present case $\frac{E_{f}}{E_{m}}=\frac{11.8}{0.4}, \frac{\ell}{d}=10.375, V_{f}=0.424$ and $E_{m}=0.4 \times 10^{6}$ psi 


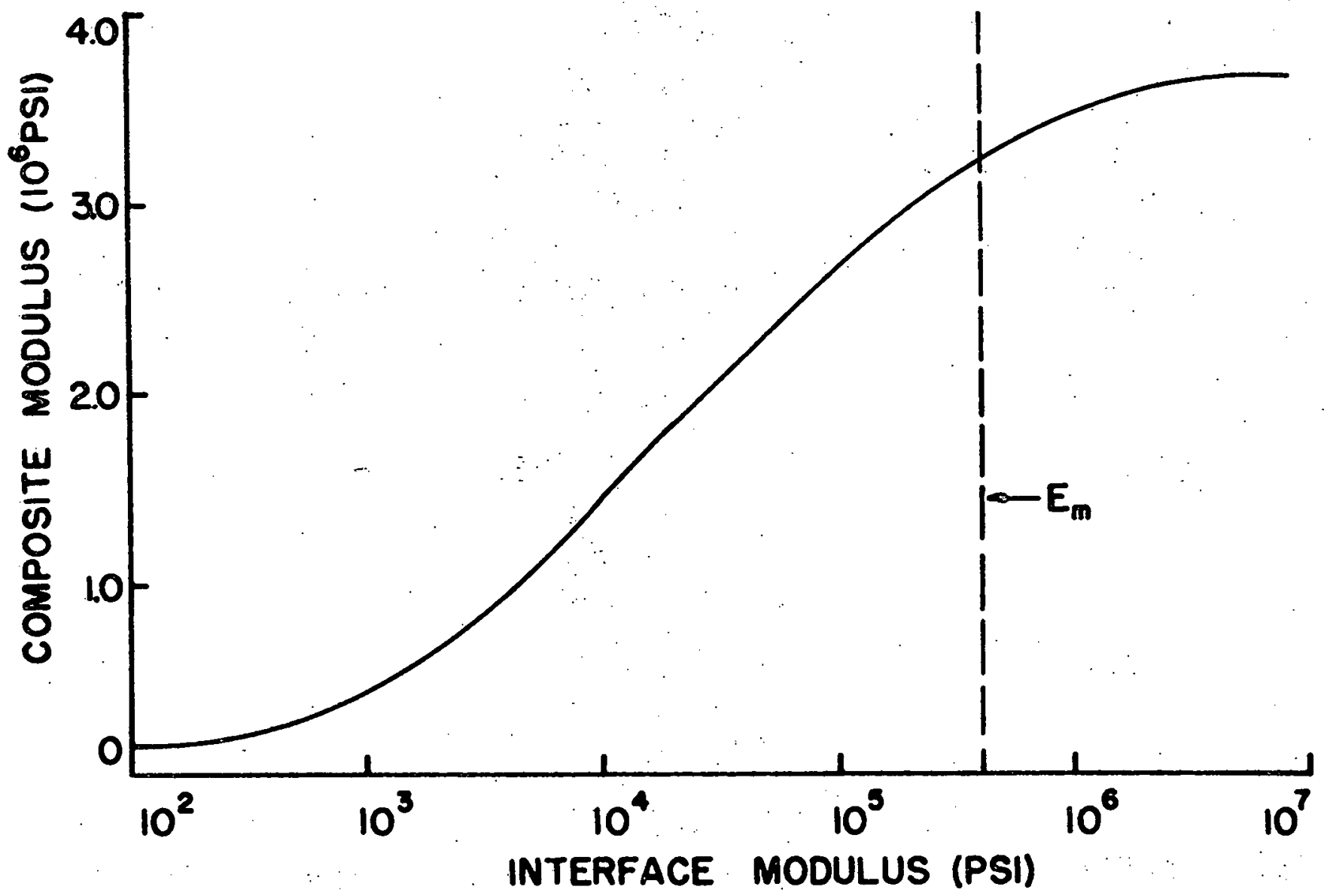

Fig. 52. Composite Modulus as a Function of Interface Modulus

\& 
thus

$$
E_{L}=3.15 \times 10^{6} \mathrm{psi}
$$

This value of the composite modulus compares favorably with the value of $3.58 \times 10^{6}$ psi obtained for the two phase composite by the finite element method. The onset of failure may be predicted from the knowledge of the stress distributions in the composite. To this end, distortion energy given as follows:

$$
U=\frac{1}{2}\left[\left(\sigma_{1}-\sigma_{2}\right)^{2}+\left(\dot{\sigma}_{2}-\sigma_{3}\right)^{2}+\left(\sigma_{3}-\sigma_{1}\right)^{2}\right]
$$

(where $\sigma_{1}, \sigma_{2}$ and $\sigma_{3}$ are principal stresses) was calculated for all the elements (Fig. 40) for different interface moduli (normalized stresses were used for this calculation). Maximum distortion energy in any element of the matrix has been plotted as a function of interface modulus in Fig. 53. Maximum distortion energy always occurs in an element near the fiber end. Strength of the composite based on von Mises failure criterion (i.e., the initiation of composite failure occurs as soon as the distortion energy in any element of the matrix reaches a limiting value) is shown qualitatively in Fig. 54. The actual strength values will depend upon the matrix strength. For very low interface modulus the strength of the composite is low due to high stress concentrations at the discontinuity. Strength of the composite increases as the interface modulus increases. But as the interface modulus changes from $10^{4}$ psi to $8 \times 10^{6}$ psi, the composite strength remains almost constant. However, Fig. 52 shows a significant change in the composite modulus over this range of interface modulus. Thus, the ultimate elongation of the composite can be controlled without affecting the failure stress by suitably controlling the interface modulus (using a surface treatment on the fibers during manufacturing). This also shows that a good combination of the tensile strength and toughness (or the impact strength) may be obtained by suitably selecting the interface properties. 


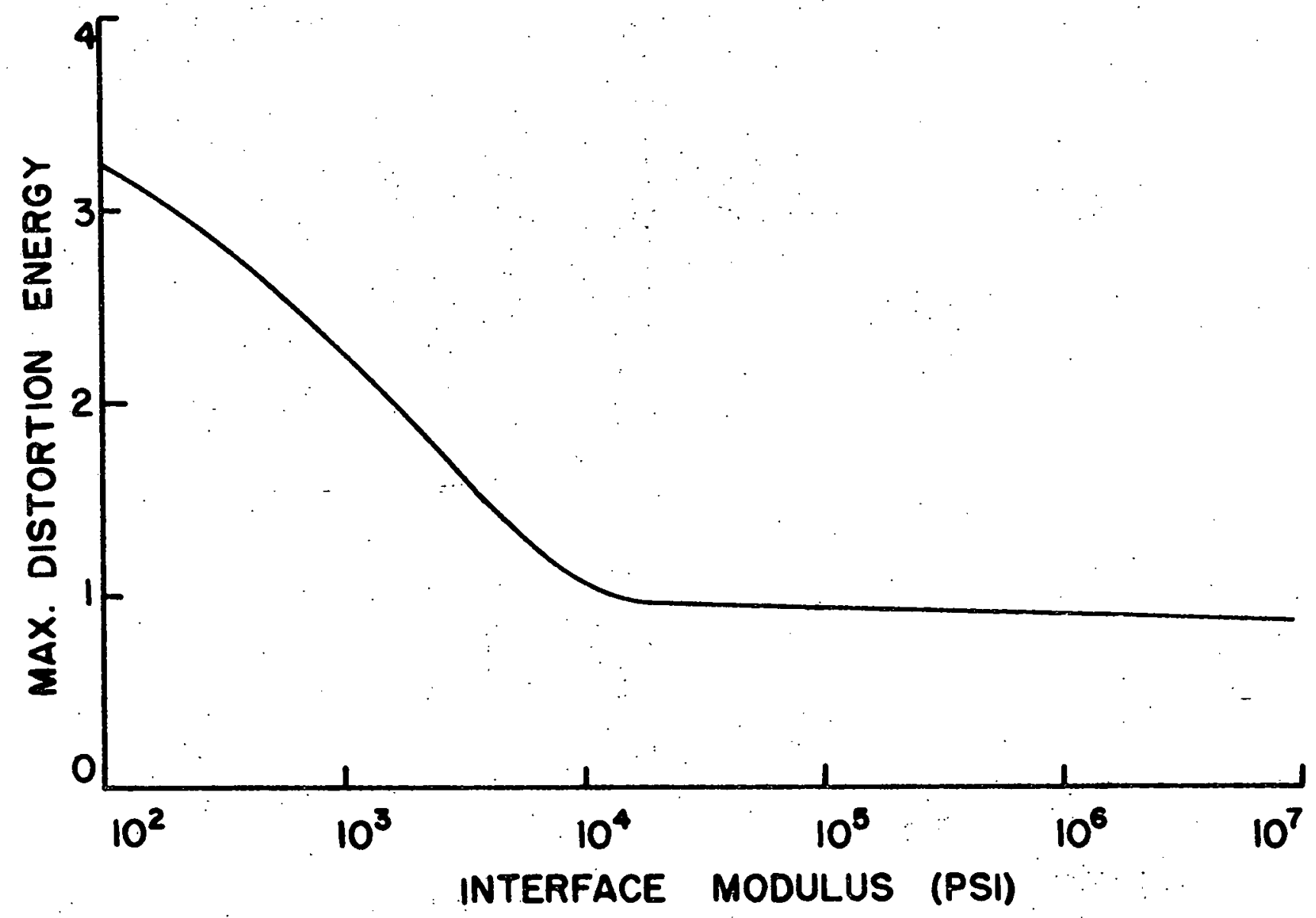

Fig. 53. Maximum Distortion Energy as a Function of. Interface Modulus 


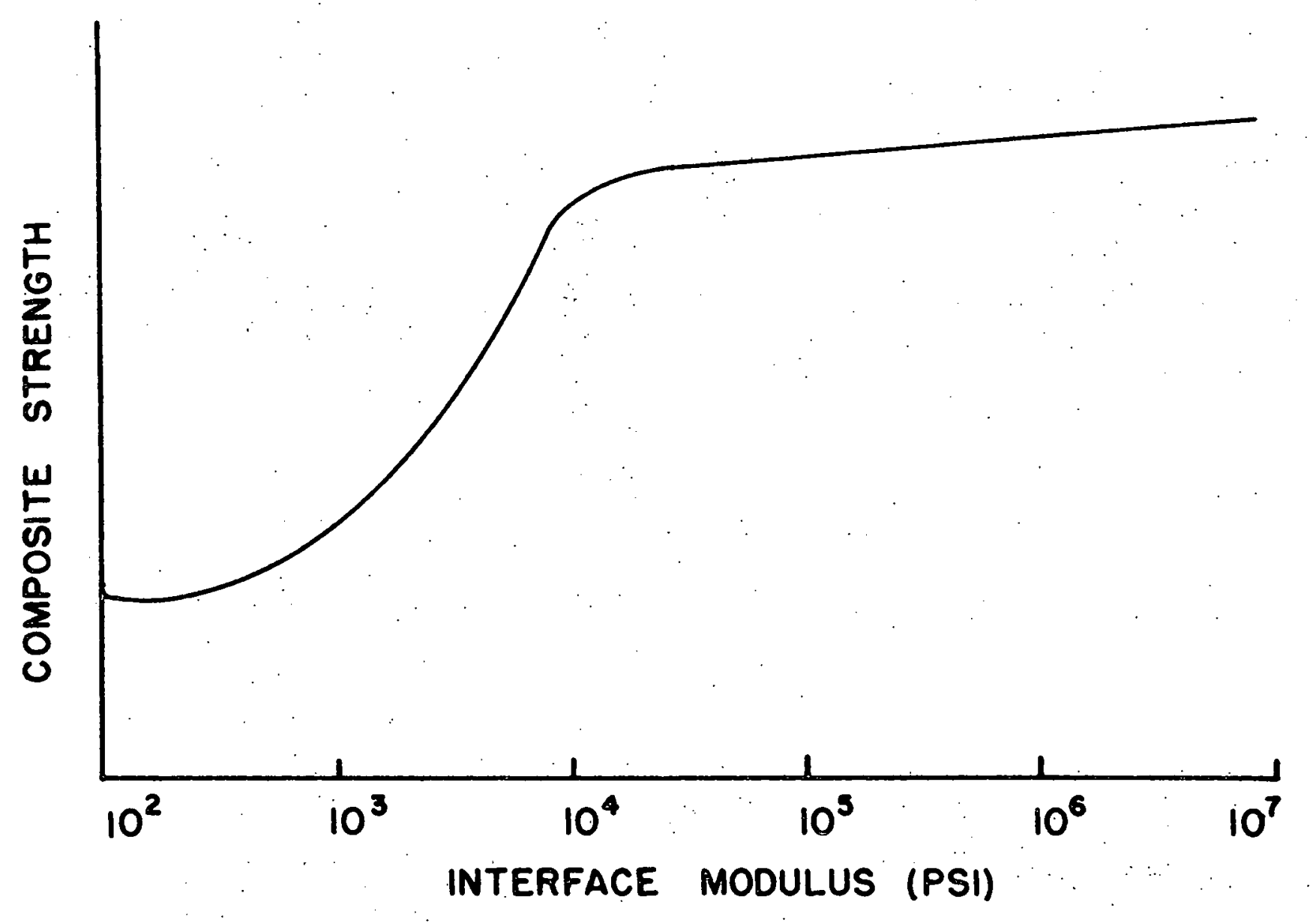

Fig. 54. Composite Strength as a Function of Interface Modulus: (Qualitative Representation) 


\section{Effect of Fiber End Condition}

It has now been shown that a very low modulus of the interface does not allow for load transfer across the interface. This fact has been made use of in studying the stress distribution in a composite with strong interface but with the fiber end debonded from the matrix. Elements of the interface adjacent to the fiber end were assigned a modulus of 100 psi while the rest of the interface had a modulus of $8 \times 10^{6}$ psi. Studies with the debonded fiber end have been carried out for two thicknesses of the interface $i . e$. with interface volume of 3.79 and 7.76 percent. Resulting stress distributions are shown in Figs. 55-58.

Axial stress distribution in the fiber (Fig. 55) shows that the stress at the fiber end is significantly reduced due to debonding of the end. The stresses increase very rapidly away from the end. This is because very high shear stress is developed in the interface near the end (Fig. 56). The high shear stress is produced due to the discontinuity at the end. The strong interface allows an efficient transfer of load from the matrix to the fibers. The fiber axial stress reaches a constant value in less than two fiber diameters from the end and the shear stress drops to zero in the same distance. These results are in agreement with the observations of Chen and Lavengood $(30)$ that debonding at the fiber ends tends to increase the maximum interfacial shear but has relatively little effect on the maximum fiber stress. Figs. 55 and 56 also show that the change in interface thickness has very little effect on the stress distributions.

Variation in the radial direction of matrix axial stress (in the elements adjacent to the fiber end, refer Fig. 42) shows (Fig. 57) that the axial stress vanishes for $\frac{r}{r_{2}}$ less than one. This is to be expected because it acts as a free boundary due to debonding of the fiber end. The axial stress increases very sharply near $\frac{r}{r_{2}}=1$. The variation of matrix axial stress along the fiber length is shown in 


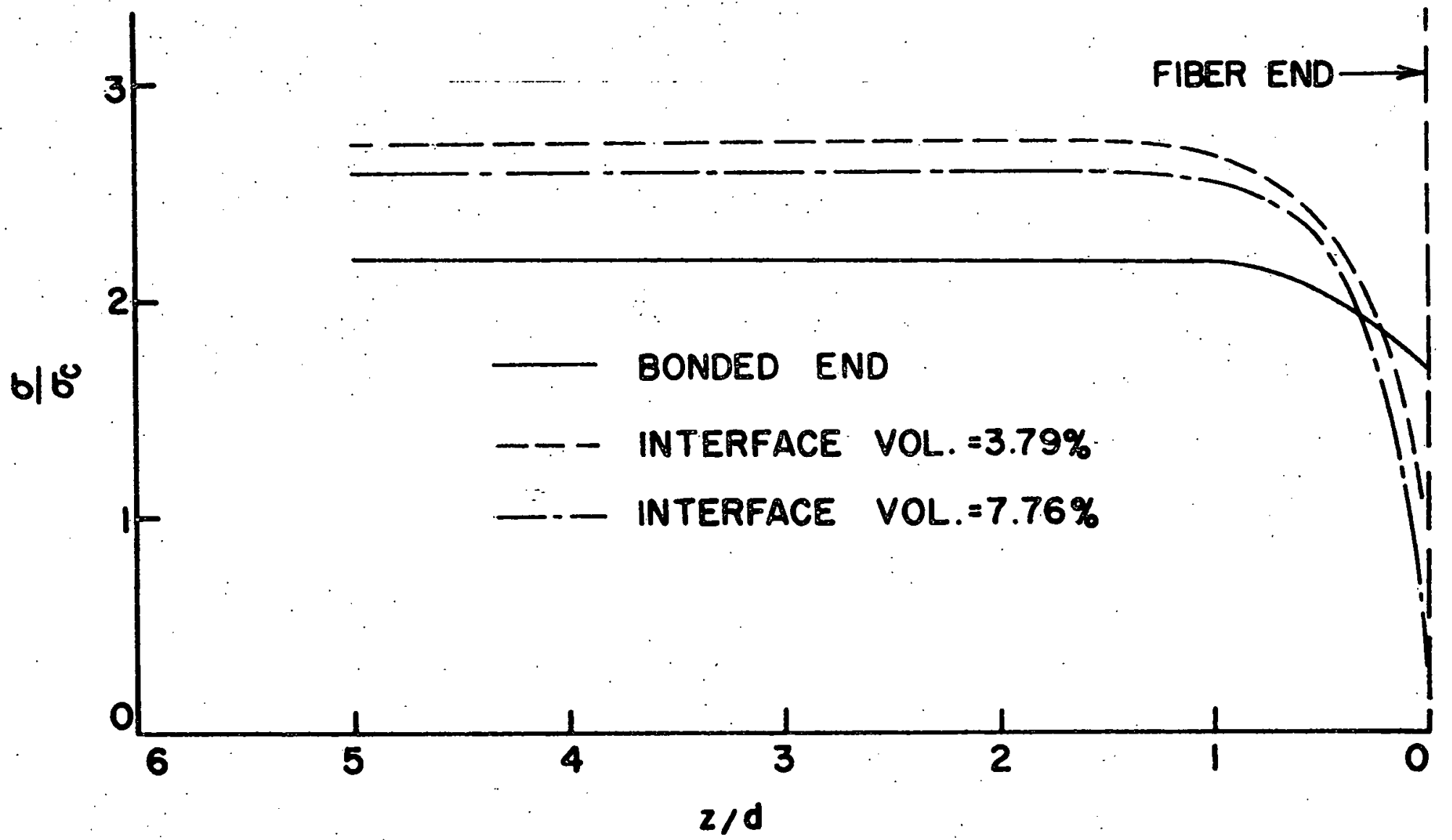

Fig. 55. Normalized Fiber Axial Stresses Along Fiber Axis for Different Fiber End Condipions 


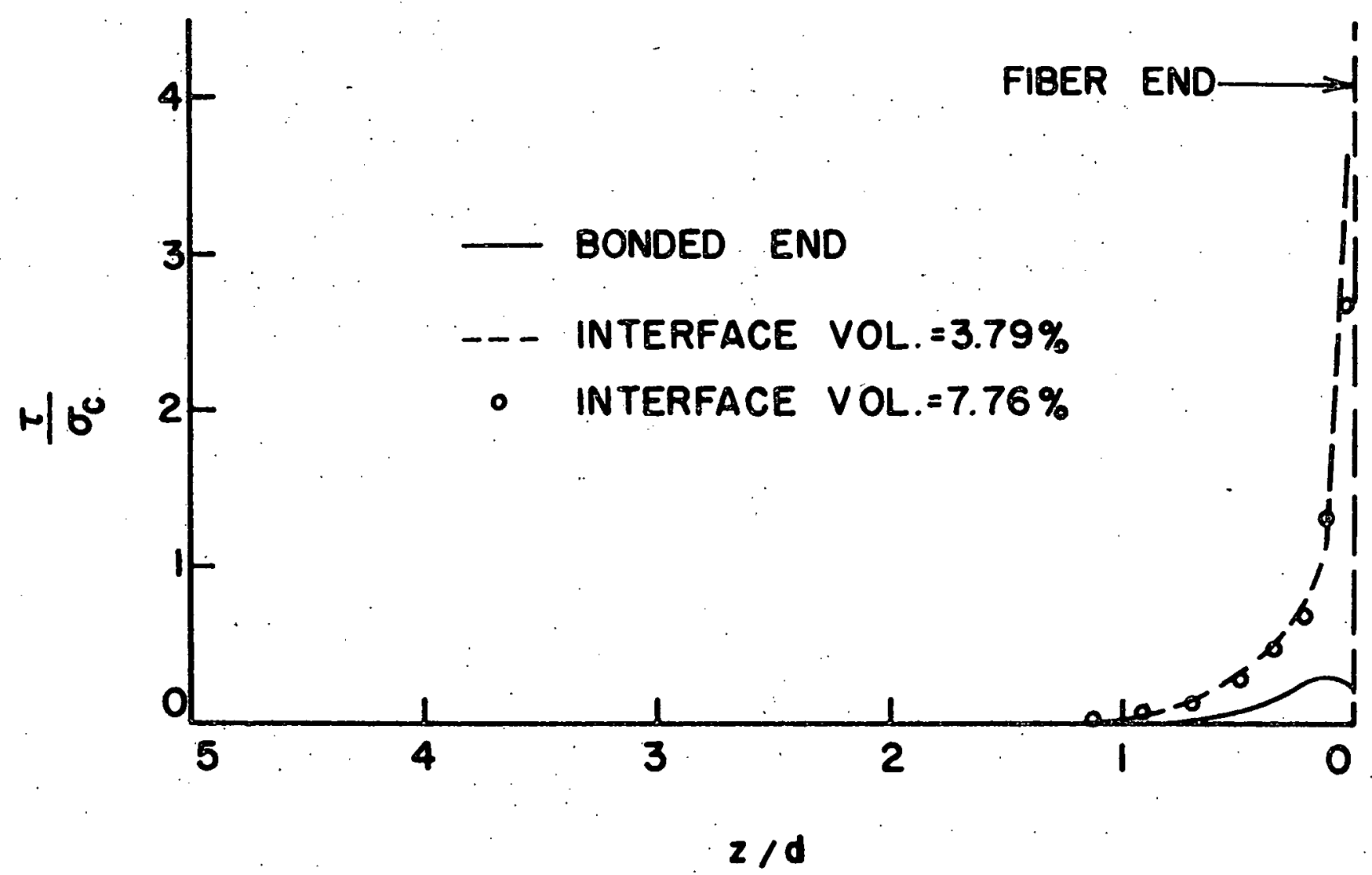

Fig. 56. Normalized Inferfacial Shear Stresses Along Fiber Axis for Different Fiber End Conditions 


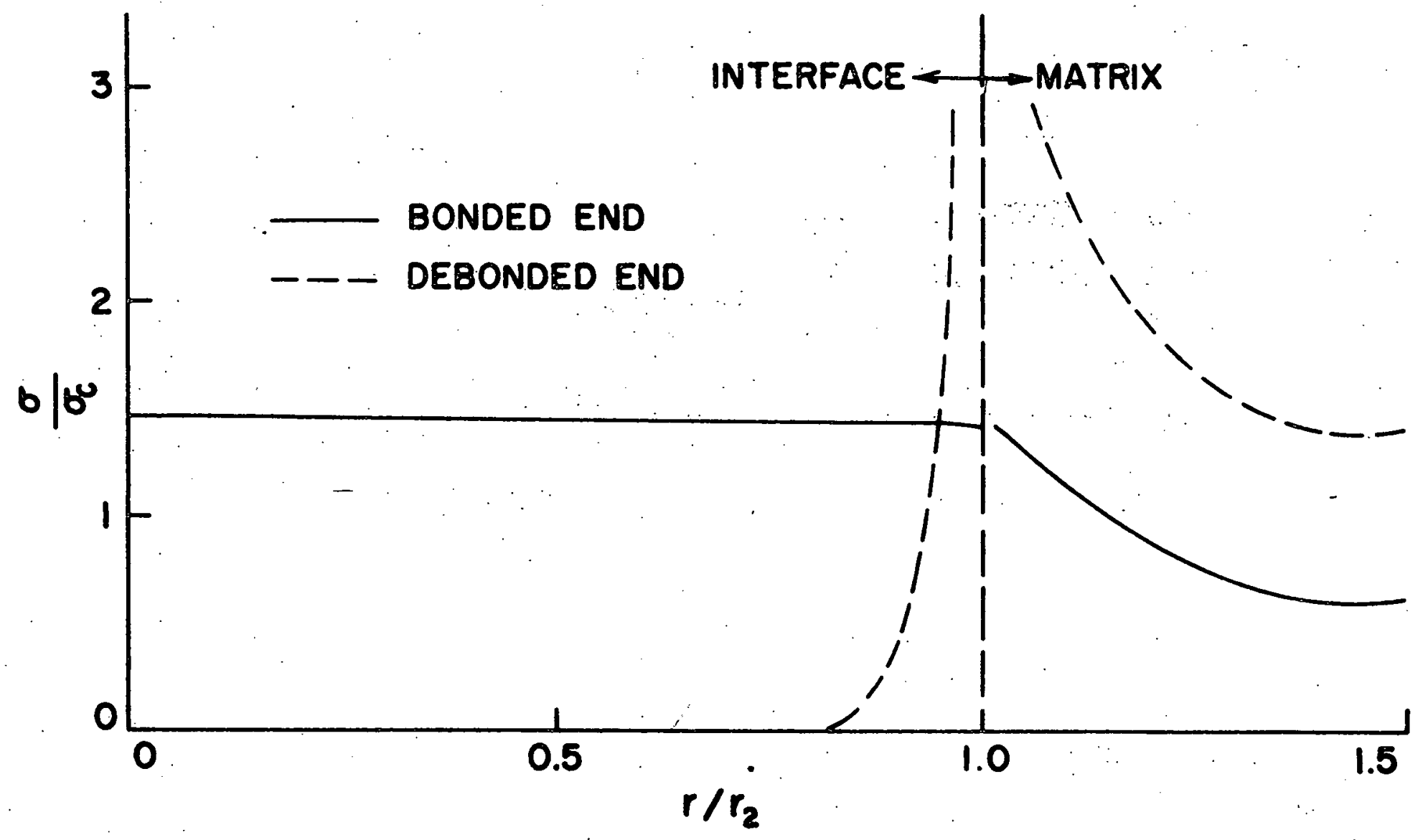

Fig. 57. Radial Variation of Axial Stresses Near Fiber End for Different Fiber End Conditions 


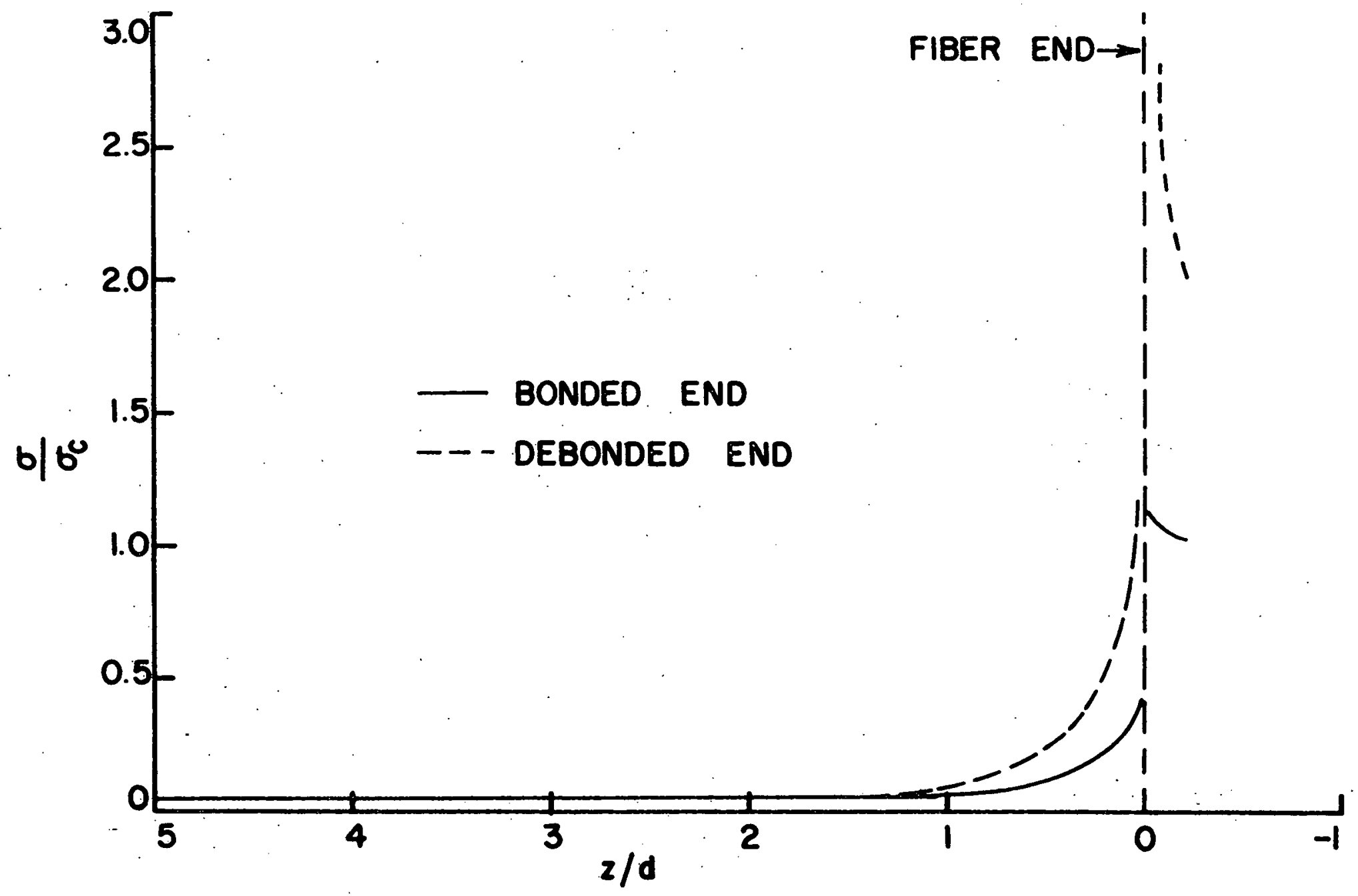

Fig. 58. Normalized Axial Stresses Along Fiber Axis for Different Fiber End Conditions: 
Fig. 58. This shows the stress increases very sharply near the fiber end and the discontinuity in the stress at this point is much severe than the one with the bonded fiber end.

Some Comments on the Results

Results of the elastic analysis of three phase fibrous composites have been presented in this section. Various stress distributions should give an insight for their relative importance and their application. A close examination of Fig. 46 shows that the maximum shear stress at the interface is about 0.4 times the applied stress and Fig. 45 shows that the maximum shear stress in the fiber is 2.5 times the applied stress $i . e$. the shear stress is approximately one sixth of the fiber stress. For a typical fiber strength of 150,000 psi, the shear stress produced in the matrix will be 25,000 psi. If no yielding is allowed, the interface would fail at this high shear stress and hence may be the controlling factor for the composite strength.

The shear stresses in case of debonded fiber ends are extremely high (Fig. 56). These stresses are high enough to cause the complete debonding of the fiber from the matrix. Debonded fiber end represents a case of very practical importance because the fiber end may be debonded due to high stresses at the interface or one broken fiber would produce two debonded fiber ends. A fiber once debonded from the matrix does not usually carry any load and its load has to be shared by other fibers. This results in an increase in the stress in other fibers and therefore may trigger the failure of other fibers and ultimately the composite. However, sometimes compressive residual stresses are present in the matrix which ore produced during manufacturing of the composite. The radial compressive stresses at the interface would cause a mechanical friction between the fiber and the matrix. Under these conditions, a fiber which has been chemically debonded from the matrix may still carry some load. The present analysis does not take into account 
the residual thermal stresses of the composite. A separate analysis has to be carried out whenever these stresses are deemed significant. Another limitation to the present analysis is the assumption of elastic behavior of the matrix. This assumption is valid in the case of some polymer matrices such as the epoxy resins. In case of metallic matrices, where the plastic flow may be quite significant, the elastic analysis has a very limited application. Behavior of the composites with the yielding matrices can be understood only by an elastic-plastic analysis. This has been the subject of investigations in the next section. 


\section{ELASTIC-PLASTIC ANALYSIS OF FIBROUS COMPOSITES}

Introduction

The behavior of a composite material can be divided into three regimes: (1) linear elastic response up to the elastic limit of the matrix material, (2) inelastic behavior beyond the elastic limit and up to that loading at which first failure occurs locally, and (3) subsequent crack propagation and total composite failure.

So far in the thesis, behavior of the composite materials has been investigated only in the first regime (i.e. with linearly elastic matrix) which has received greater attention of other investigators than the other two regimes. But due to the assumption of a linearly elastic matrix material, these studies are applicable in predicting the behavior of the composite material with very special matrix material for example the epoxies or a ceramic or in predicting the initial (elastic or Young's) modulus of the composite and elastic stress concentrations.

However, a number of materials, for example metals and polymers, selected as the matrix materials for the composites exhibit high strain capability i.e., high ductility or yielding. This ductility permits large local strains to occur in matrix near fiber discontinuities and in regions between closely spaced fibers. But since elastic analyses do not permit this local yielding they are only valid up to the applied composite stress at which the most stressed material region reaches the elastic limit value. And this applied stress level is typically only a fraction of that at which composite failure occurs.

The consideration of nonlinear material response represents a somewhat more formidable undertaking. As a result, not much work in the general area of microme chanical behavior of composite materials, $i . e_{.}$, consideration of local states of stress as affecting gross or composite properties, has been done. Doughty and 
McGarry ${ }^{(31)}$ studied a single fiber model of a discontinuous fiber reinforced composite material with an elastic-plastic and viscoelastic matrix. They show that even at the composite stress lower than the yield stress of the matrix stress concentration at the fiber end causes some local yielding in the matrix, the extent of which depends on the shape of the fiber end. This yielding has a profound effect on the stress in the fiber end. Adams ${ }^{(32)}$ carried out an inelastic analysis of a unidirectional composite subjected to transverse normal loading. His results indicate that extensive local yielding and redistribution of stresses can occur in a composite with very little indication of such behavior being apparent by observation of the total stress-strain response of the composite alone. These studies show the importance of elastic-plastic analyses to the understanding of composite material behavior. However, much work has to be done before the design criteria and theories of failure could be established and interpreted in the light of elastic plastic analyses of the composite material. The present elastic-plastic analysis of fibrous composite subjected to longitudinal (axial) loading is a step in that direction.

\section{Analysis of the Models}

Packing of the fibers in the composites was assumed to be the same as in Section IV (Fig. 38). It was pointed out that this idealization of the composite has a definite limitation since in the region between the two rows of fibers the total load is supported by the matrix. Such a section would, therefore, be the weakest and hence the controlling one for the strength of the composite. An elastic-plastic analysis would show that this section would be the first to yield and the stresses in this section can not build up beyond a certain value although the strains may be very large. Thus, the applied stress on the composite studied by this model (Fig. 38) is limited by the section between the two rows of fibers. 


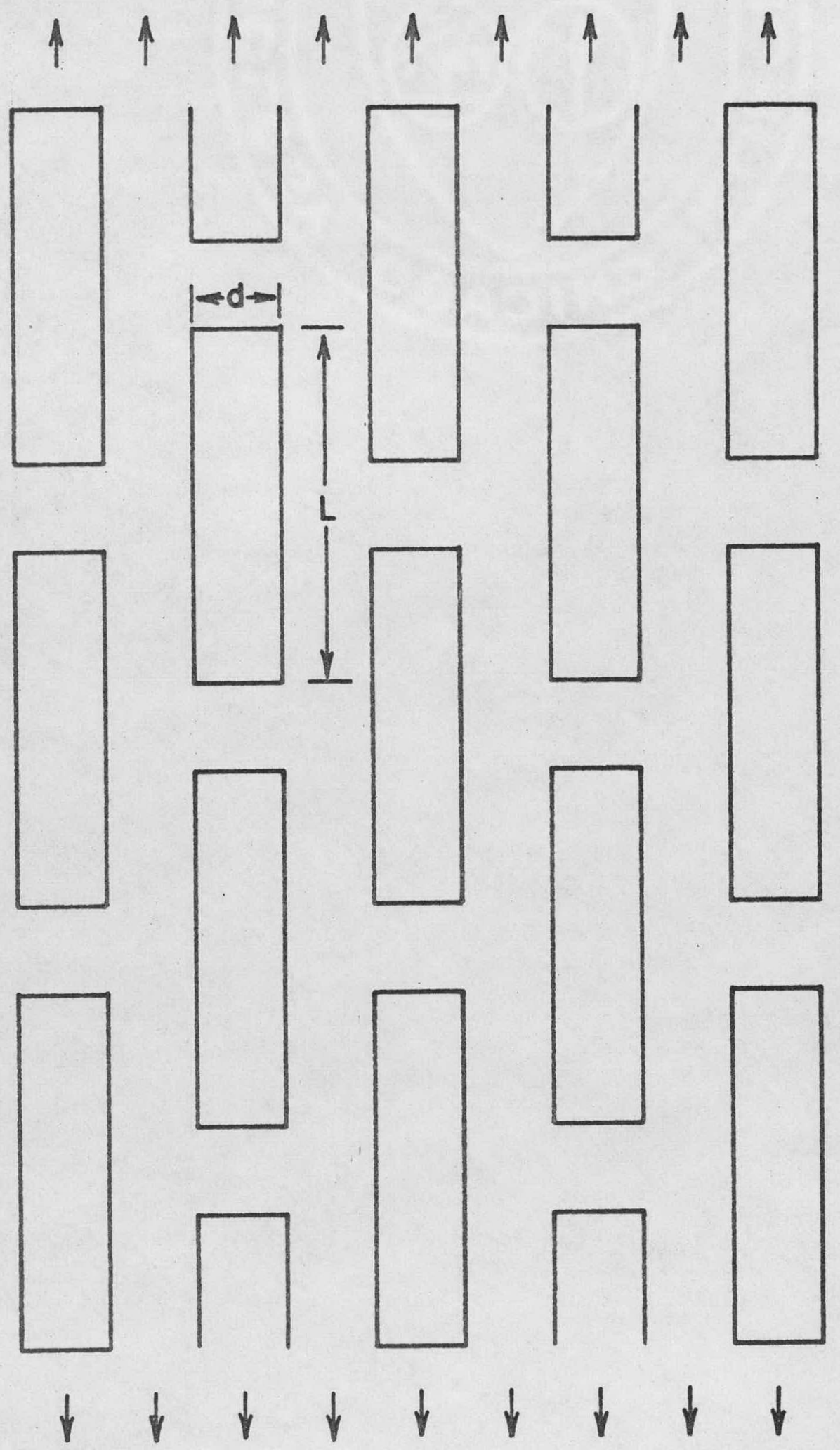

Fig. 59. Packing of Discontinuous Fibers in a Composite 


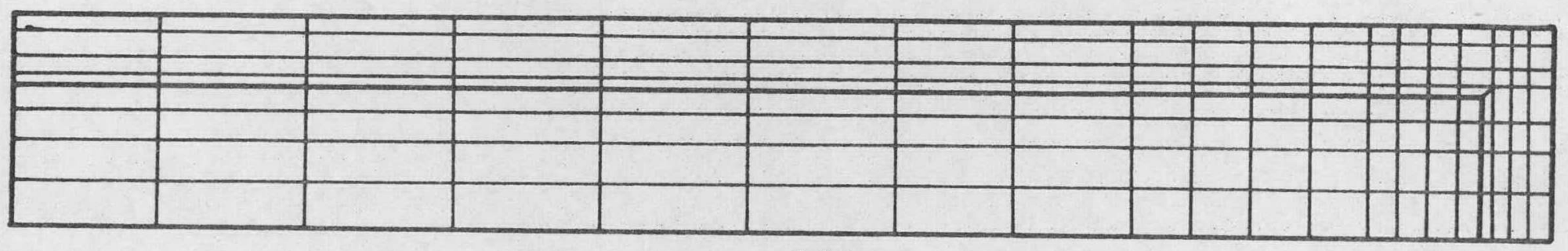

(A)

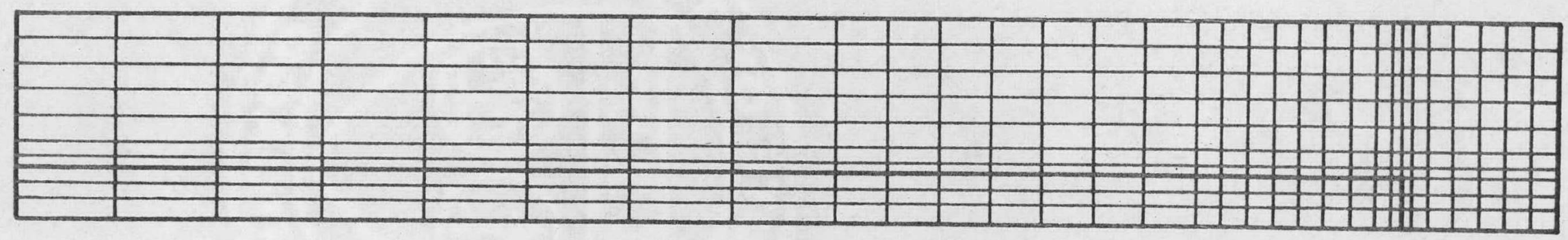

(B)

Fig. 60. Finite Element Grids for (A) $r_{2} / r_{1}=0.67,(B) r_{2} / r_{1}=0.25$ 
boundary conditions for elastic analysis, can no longer be used due to the elasticplastic matrix. The following procedure was used to satisfy the boundary conditions:

The stress and displacement distribution is found (with reference to Fig. 39) such that

$$
\begin{aligned}
& \left(U_{z}\right)_{A B}=K_{1} \text { (Specified displacement in z-direction), } \\
& \left(U_{z}\right)_{D C}=0 \text { (Symmetry), } \\
& \left(U_{r}\right)_{B C}=K_{2} \text { (Specified displacement in r-direction), } \\
& \left(U_{r}\right)_{A D}=0 \text { (Symmetry), } \\
& \left.\tau_{r z}=\tau_{z r}=0 \text { (on } A B C D\right) .
\end{aligned}
$$

From these boundary conditions, $\sigma_{r}$ and $\sigma_{z}$ in all elements and displacements $U_{r}$ and $U_{z}$ at all nodal circles are determined. $K_{1}$ is selected to obtain the desired value of strain in $z$-direction. The value of $K_{2}$ has to be picked such that the net force in the r-direction along $B C$ is zero. Thus

$$
\left(F_{r}\right)_{B C}=\int_{B C} \sigma_{r} d z=|B C| \bar{\sigma}_{r}=0
$$

Several values of $K_{2}$ may have to be tried to satisfy equation (1). Experience shows that it usually takes three attempts to get the correct value to $K_{2}$ which will satisfy equation (1). With this value of $K_{2}$, the solution obtained is the desired one which satisfies all the boundary conditions and is equivalent to the superimposed solution in the case of elastic analysis.

It was pointed out that with the fiber packing shown in Fig. 38, the stress in a single fiber builds up due only to the transfer of load from the matrix. The above imposed boundary condition that $\tau_{r z}$, vanishes on $B C$ does not allow any stress transfer from one fiber to the other and hence is invalid for the fiber packing of Fig. 59. 
The distribution of $\tau_{r z}$ is not known. A close approximation of the actual situation would be to assume that the axial strain at each point of the outer boundary (BC) of the representative cell (Fig. 39 ) is the same as the average composite axial strain. This means that instead of shear stresses, the tangential displacements are prescribed on BC. The stress and displacement distribution is found (with reference to Fig. 39) which satisfies the following boundary conditions:

$$
\begin{aligned}
& \left(U_{z}\right)_{A B}=k_{1} \text { (Specified displacement in z-direction), } \\
& \left(U_{z}\right)_{D C}=0 \text { (Symmetry), } \\
& \left(U_{r}\right)_{B C}=k_{2} \text { (Specified displacement in r-direction), } \\
& \left(U_{r}\right)_{A D}=0 \text { (Symmetry); } \\
& \tau_{r z}=\tau_{z r}=0 \text { on } A B, D C \text { and } A D \\
& \left(U_{z}\right)_{B C}=U(z) \text { (Specified tangential displacement at all nodal } \\
& \text { circles on } B C) .
\end{aligned}
$$

$U(z)$ for each nodal circle on $B C$ is prescribed such that the axial strain along $B C$ is constant and is equal to the one produced by displacement $k$; on $A B$. It can be easily seen that $U(z)$ at a nodal circle on $B C$ is given by

$$
U(z)=\frac{k_{1} \cdot z}{|B C|}
$$

where $z$ is the distance of the nodal circle from DC whose normal displacement is zero. Values of $k_{1}$ and $k_{2}$ are picked in the manner discussed earlier. The stresses and displacments thus obtained represent the state of stress in a composite with the fiber packing shown in Fig. 59. 
Stresses With Axial Strains Imposed on One Boundary of the Representative Cell

To calculate the stresses, the following component properties were assumed:

$$
\text { Fibers } \begin{aligned}
E & =11.8 \times 10^{6} \mathrm{psi} \\
\nu & =0.197 \\
\mathrm{E} & =0.4 \times 10^{6} \mathrm{psi} \\
\nu & =0.35 \\
\text { Yield Stresses } & =8,000 \text { and } 20,000 \text { psi } \\
\text { Yield Strains* } & =2.0 \text { and } 5.0 \text { percent } \\
\text { Post Yield } E & =100 \text { psi }
\end{aligned}
$$

The stress distributions for the composite with the fiber packing shown in Fig. 38 are discussed in this section. The stress distributions for the second fiber packing will be discussed in the next section.

The stress distributions for $r_{2} / r_{1}=0.67$ and matrix yield stress of 8,000 psi are shown in Figs. $61-64$ for four composite strain le vels namely $0.2,0.5,1.0$ and 2.0 percent. The average composite stresses are given in Table 6. Variation of the axial stress in the fiber is shown in Fig. 61. General nature of the curves is the same as shown in Fig. 41 for elastic analysis. Fiber stress attains a maximum value in about two fiber diameters from the fiber end. As the composite strain increases the maximum fiber stress increases. When the composite strain increases from 1.0 to 2.0 percent, the increase in maximum fiber stress is very small.

* In case of triaxial stresses, the von Mises criterion for yielding has been used. An effective strain $\varepsilon$ is determined according to the formula:

$$
\varepsilon_{e}=\sqrt{\frac{1}{2}\left[\left(\varepsilon_{1}-\varepsilon_{2}\right)^{2}+\left(\varepsilon_{2}-\varepsilon_{3}\right)^{2}+\left(\varepsilon_{3}-\varepsilon_{1}\right)^{2}\right]}
$$

(where $\varepsilon_{1}, \varepsilon_{2}$ and. $\varepsilon_{3}$ are principal strains) for every element. If $\varepsilon_{e}$ for an element is greater than the yield strain of the material then that element is considered yielded. 


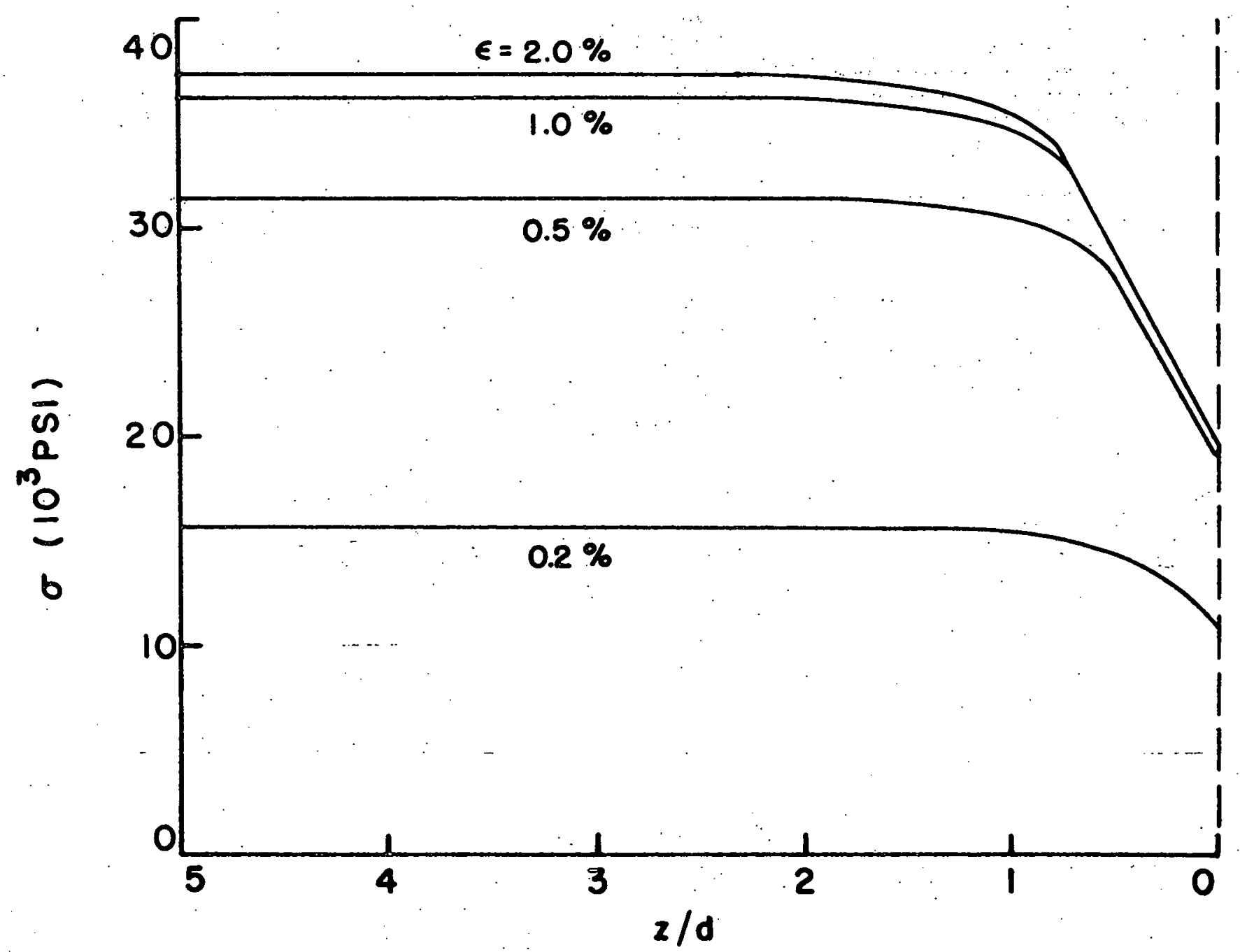

Fig. 61. Fiber Axial Stresses Along Fiber Axis $\left(r_{2} / r_{1}=0.67\right.$, Matrix Yield Strain $=2.0 \%$, Axial Strain Imposed on One Boundary) 


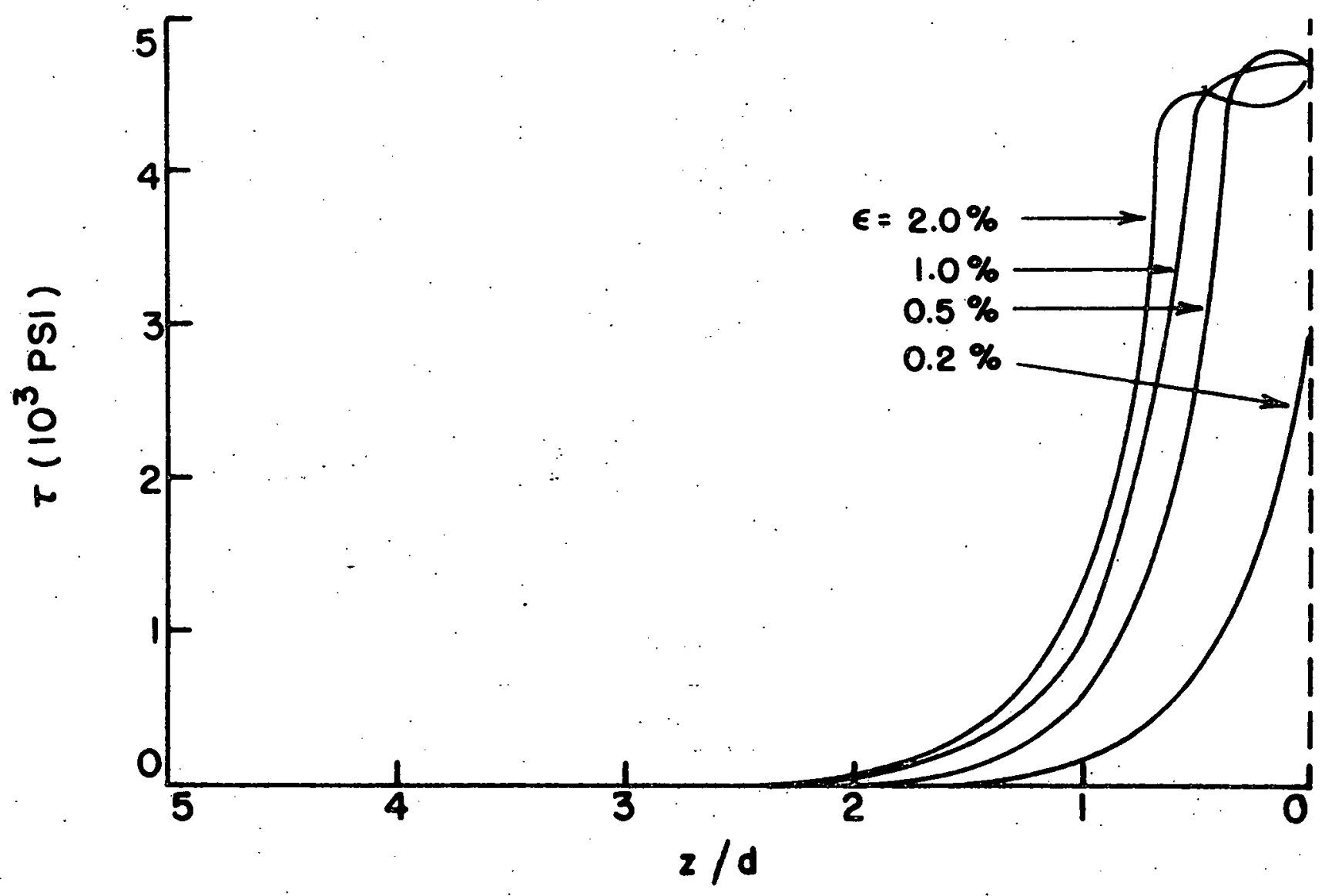

Fig. 62. Interfacial Shear Stresses Along Fiber Axis $\left(r_{2} / r_{1}=0.67\right.$, Matrix Yield Strain $=2.0 \%$, Axial Strain Imposed on One Boundary) 


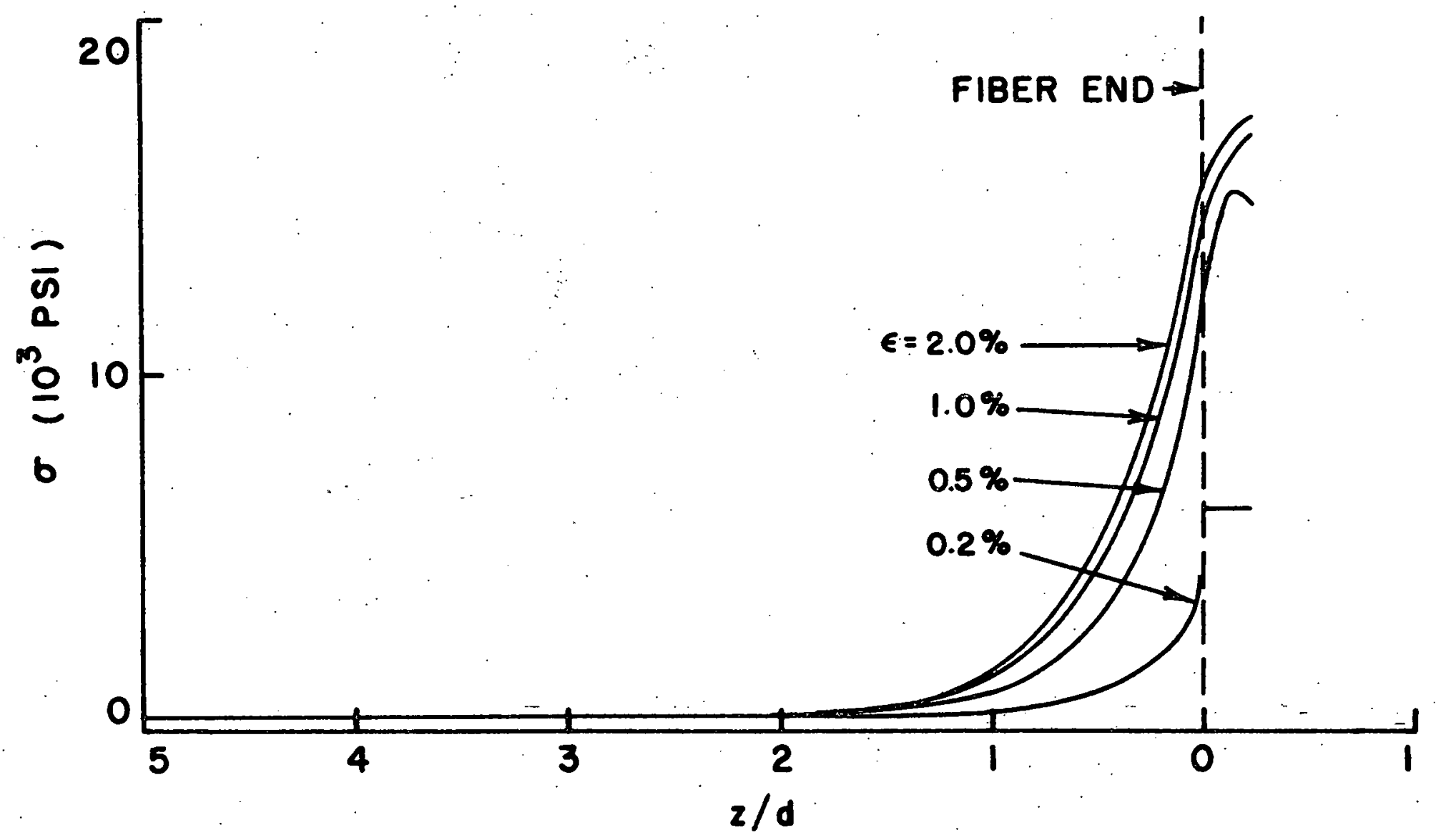

Fig. 63. Matrix Axial Stresses Along Fiber Axis $\left(r_{2} / r_{1}=0.67\right.$, Motrix

Yield Strain $=2.0 \%$, Axial Strain Imposed on One Boundary) 


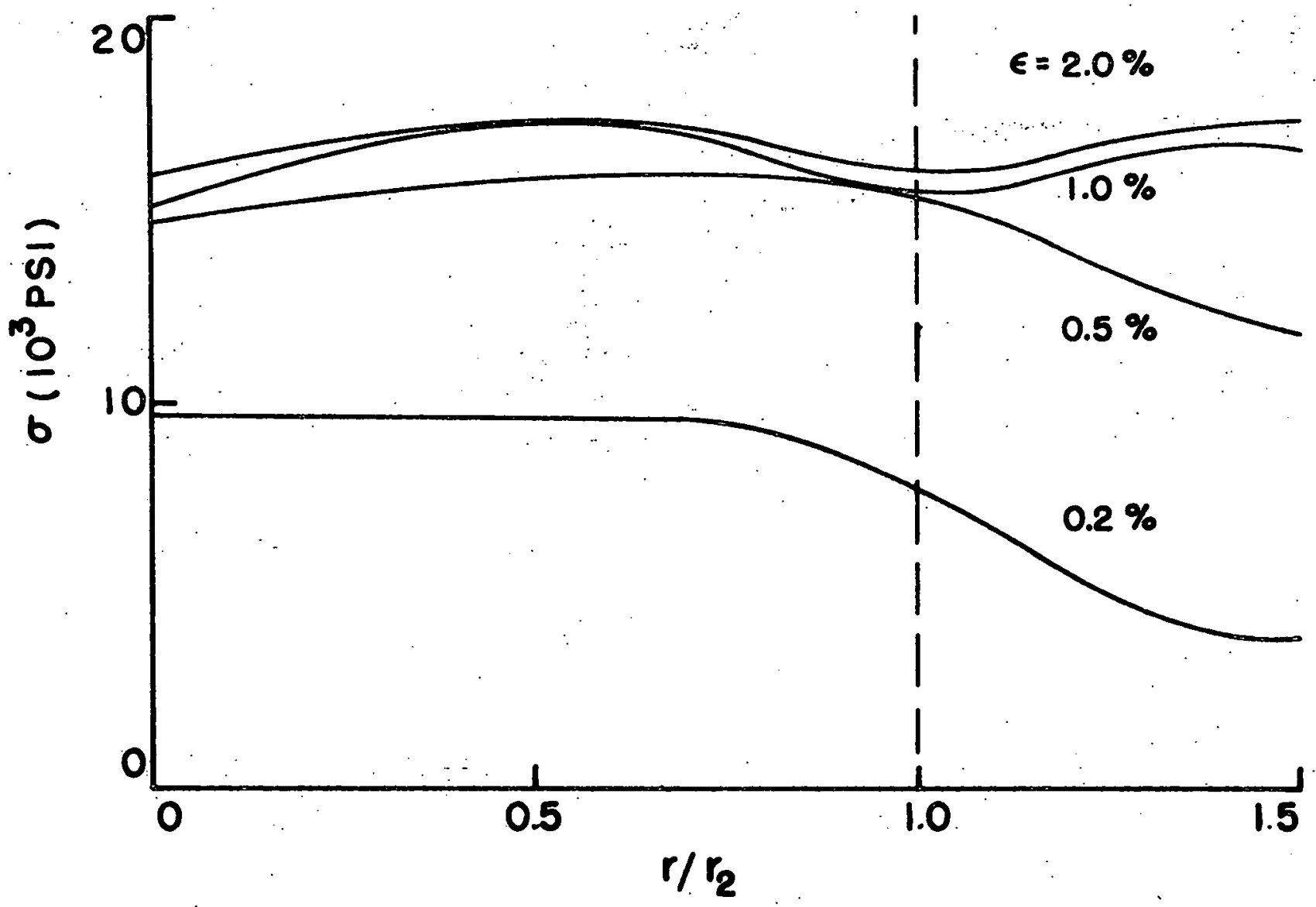

Fig. 64. Radial Variation of Matrix Axial Stress Near Fiber End $\left(r_{2}\right) r_{1}=0.67$, Matrix Yield Strain $=2.0 \%$, Axial Strain Imposed on One Boundary) 
Table 6. Average Composite Stresses for $\frac{r_{2}}{r_{1}}=0.67$ and $\left(\sigma_{y_{m}}\right)=8,000$ psi

\begin{tabular}{cccc}
\hline $\begin{array}{c}\text { Composite Axial } \\
\text { Strain }\end{array}$ & $\begin{array}{c}\text { Matrix Yield } \\
\text { Strain }\end{array}$ & $\begin{array}{c}\text { Matrix Yield } \\
\text { Stress }\end{array}$ & $\begin{array}{c}\text { Average Composite } \\
\text { Stress }\end{array}$ \\
\hline 0.2 percent & 2.0 percent & 8,000 psi & 7,160 psi \\
0.5 percent & 2.0 percent & 8,000 psi & 13,340 psi \\
1.0 percent & 2.0 percent & 8,000 psi & 16,360 psi \\
2.0 percent & 2.0 percent & 8,000 psi & 16,820 psi \\
\hline
\end{tabular}

This is because the matrix between the two fiber rows (Fig. 38) has yielded and the composite stress does not increase significantly due to this change in composite strain. Yielding of the matrix is shown in Fig. 65 where the shaded elements represent the yielded region. There are no regions of yielded matrix at 0.2 percent strain. Some elements of matrix have yielded at 0.5 percent strain. More elements yield at 1.0 percent strain. But as the composite strain is increased from 1.0 to 2.0 percent, the region in which the matrix has yielded does not increase because the weak section of the composite with matrix alone has already yielded. Fig. 61 also shows that the fiber stress increases linearly near the fiber end for composite strains of $0.5,1.0$ and 2.0 percent. This is, once again, due to the yielding of. matrix at the interface near the fiber end. Shear stresses near the fiber end are high and of nearly the same magnitude for composite strains of 0.5, 1.0 and 2.0 percent.(Fig. 62). The initial straight line for fiber stress should be expected as a result of equation ( 7 ) in Section IV.

The shear stresses at the interface are shown varying along the fiber length in Fig. 62. Shear stresses for the composite strains of $0.5,1.0$ and 2.0 percent are high near the fiber end and remain constant for some length and then very 
$\epsilon=0.2 \%$
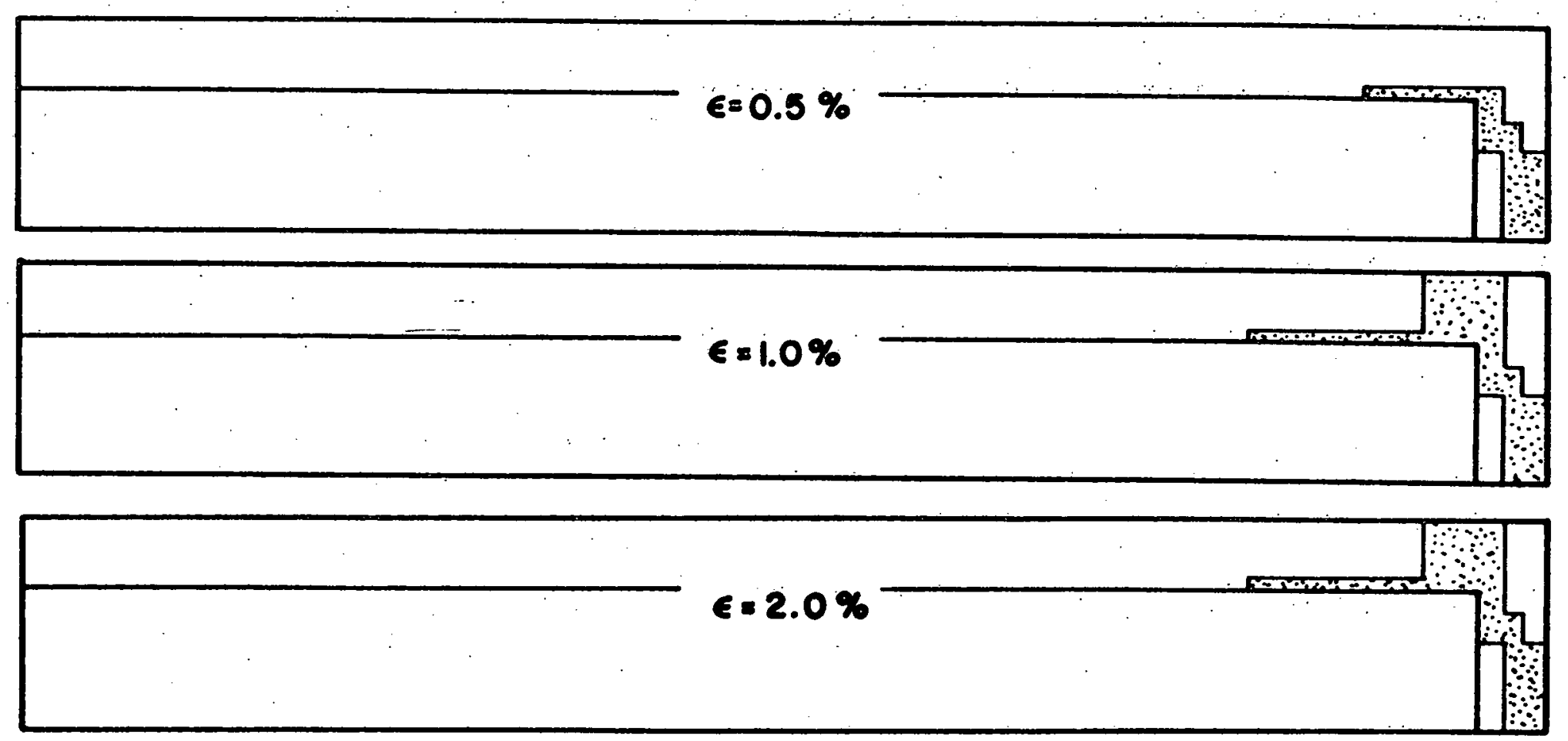

Fig. 65. Yielding of Matrix at Different Composite Strains $\left(r_{2} / r_{j}=0.67\right.$, Matrix Yield Strain $=2.0 \%$, Axial Strain (mposed on One Boundary) 
quickly drop to zero. The matrix axial stresses at the interface are plotted as a function of distance from the fiber end in Fig. 63. The stresses increase very sharply near the fiber end due to the discontinuity. Variation in the radial direction of matrix axial stress (in the elements adjacent to the fiber end, refer Fig. 42) is shown in Fig. 64. The curve for 0.2 percent composite strain is similar to one in Fig. 42 in the case of the elastic analysis. This is becouse at 0.2 percent composite strain even the highest stressed element of the matrix is elastic. However, at the composite strain of $0.5,1.0$ and 2.0 percent the matrix near the fiber end has yielded and hence the stresses do not show much variation.

The stress distributions for $r_{2} / r_{1}=0.67$ and the matrix yield stress of 20,000 psi are shown in Figs. 66-69. The growth of yield zone for this case is shown in Fig. 70. The average composite stresses are given in Table 7.

Table 7. Average Composite Stresses for $\frac{r_{1}}{r_{2}}=0.67$ and $\left(\sigma_{y}\right)_{m}=20,000$ psi

\begin{tabular}{cccc}
\hline $\begin{array}{c}\text { Composite Axial } \\
\text { Strain }\end{array}$ & $\begin{array}{c}\text { Matrix Yield } \\
\text { Strain }\end{array}$ & $\begin{array}{c}\text { Matrix Yield } \\
\text { Stress }\end{array}$ & $\begin{array}{c}\text { Average Composite } \\
\text { Stress }\end{array}$ \\
\hline 0.5 percent & 5.0 percent & 20,000 psi & 17,730 psi \\
1.0 percent & 5.0 percent & 20,000 psi & 31,810 psi \\
2.0 percent & 5.0 percent & 20,000 psi & 40,420 psi \\
4.0 percent & 5.0 percent & 20,000 psi & 41,830 psi \\
\hline
\end{tabular}

Due to higher yield stress, higher strain levels of $0.5,1,0,2.0$ and 4.0 percent were selected for the analysis. A comparison of Figs. 66-69 with the corresponding Figs. 61-64 shows that the stress distributions are very similar for the two cases of yield stress. The explanation of results for stress distributions 


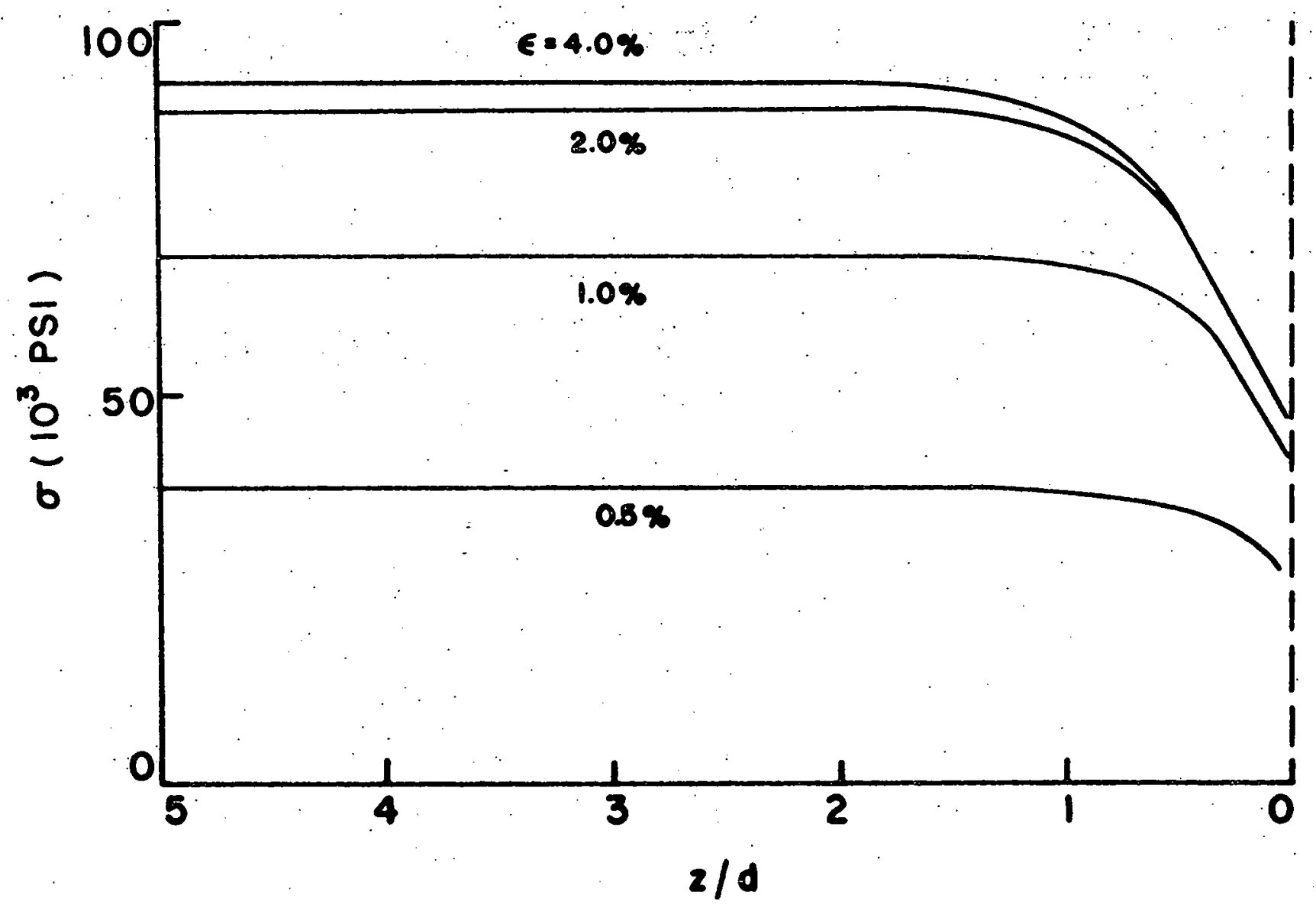

Fig. 66. Fiber Axial Stresses Along Fiber Axis $\left(r_{2} / r_{1}=0: 67\right.$, Matrix Yield Strain $=5.0 \%$, Axial Strain Imposed on One Boundary) 


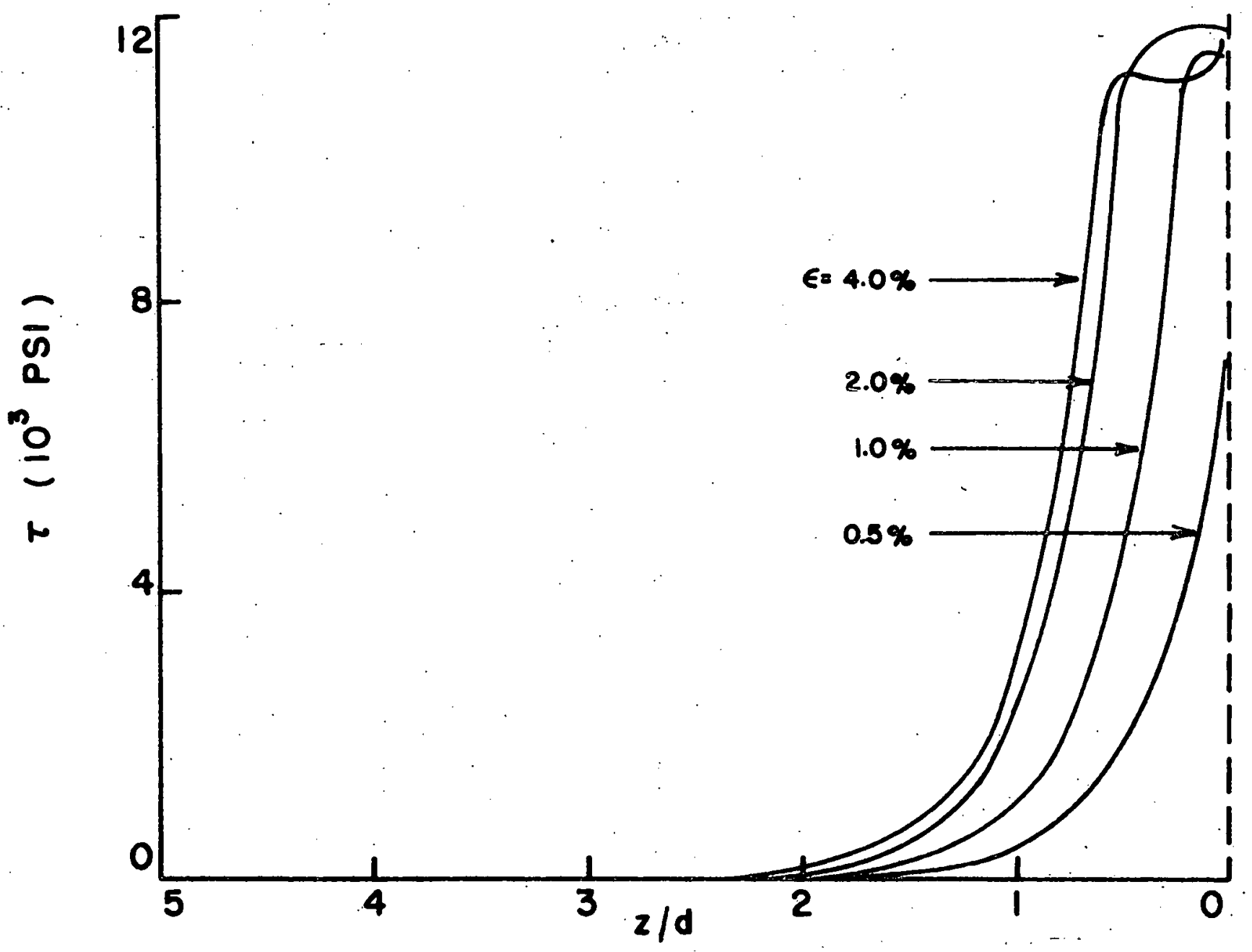

Fig. 67. Interfacial Shear Stresses Along Fiber Axis $\left(r_{2} / r_{1}=0.67\right.$, Matrix Yield Strain $=5.0 \%$, Axial Strain Imposed on One Boundary) 


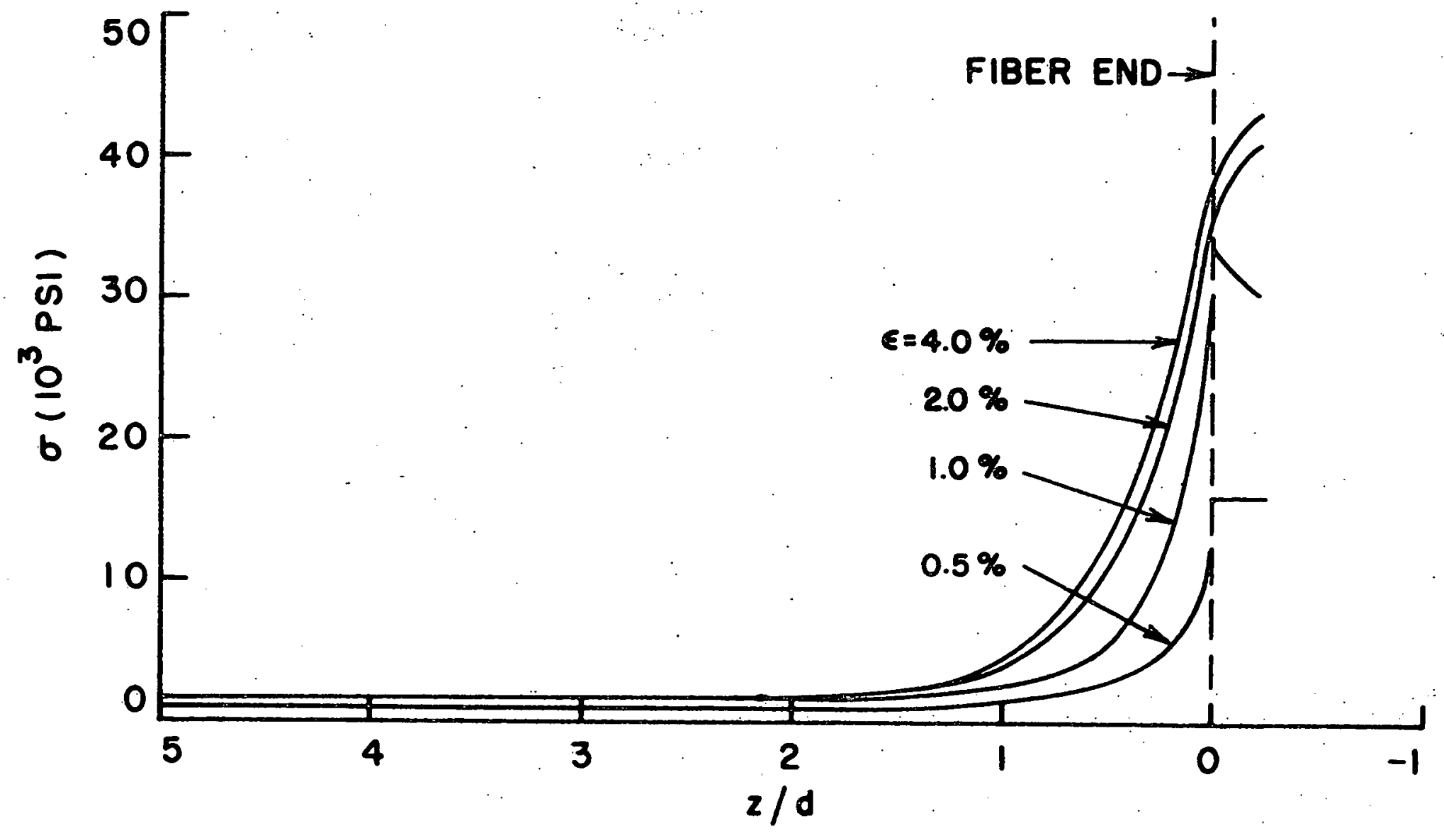

Fig. 68. Matrix Axial Stresses Along Fiber Axis $\left(r_{2} / r_{1}=0.67\right.$, Matrix Yield Strain $=5.0 \%$, Axial Strain Imposed on One Boundary) 


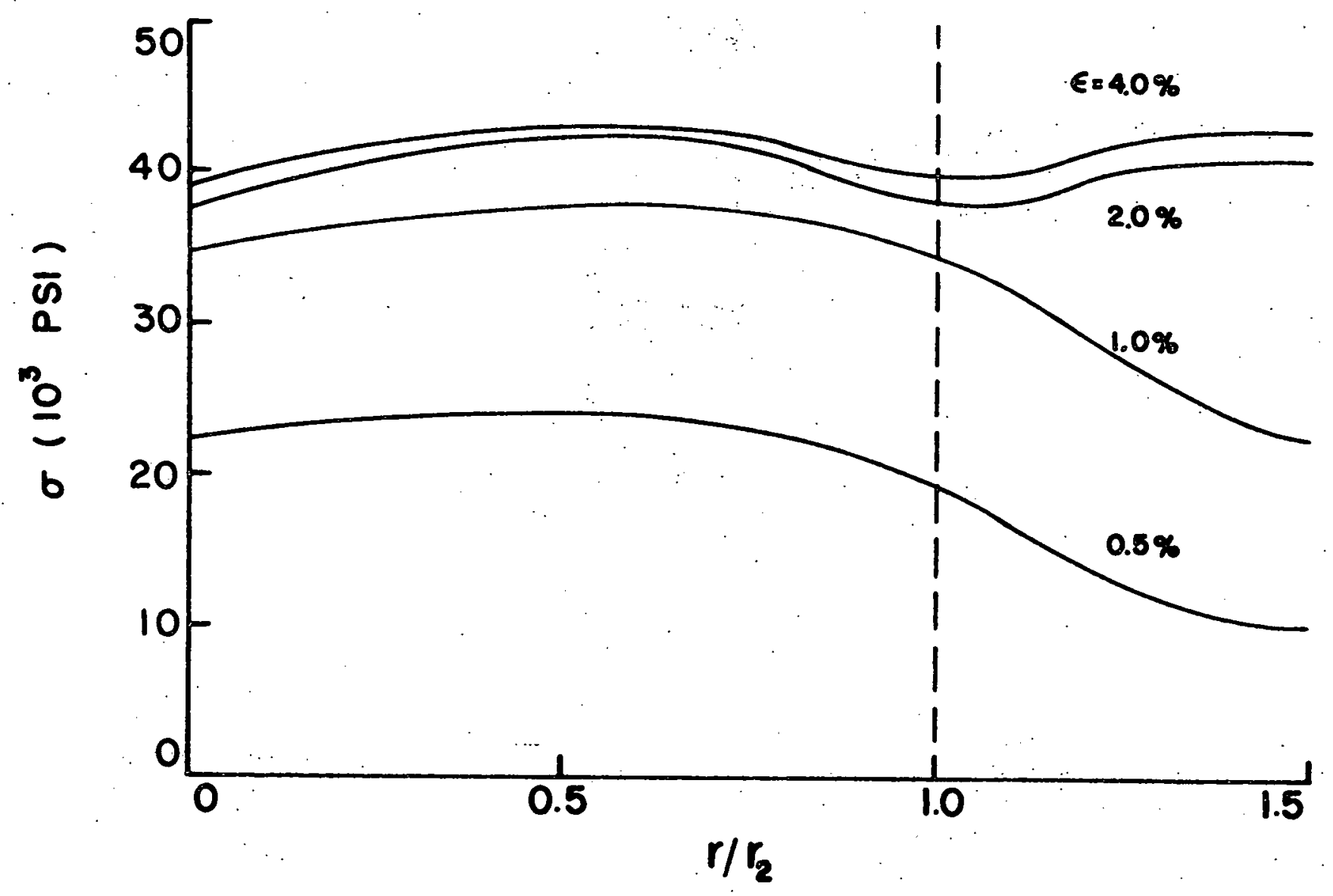

Fig. 69. Radial Variation of Matrix Axial Stresses Near Fiber End $\left(r_{2} / r_{1}=0.67\right.$, Matrix Yield Strain $=5.0 \%$, Axial Strain imposed on One Boundary) 

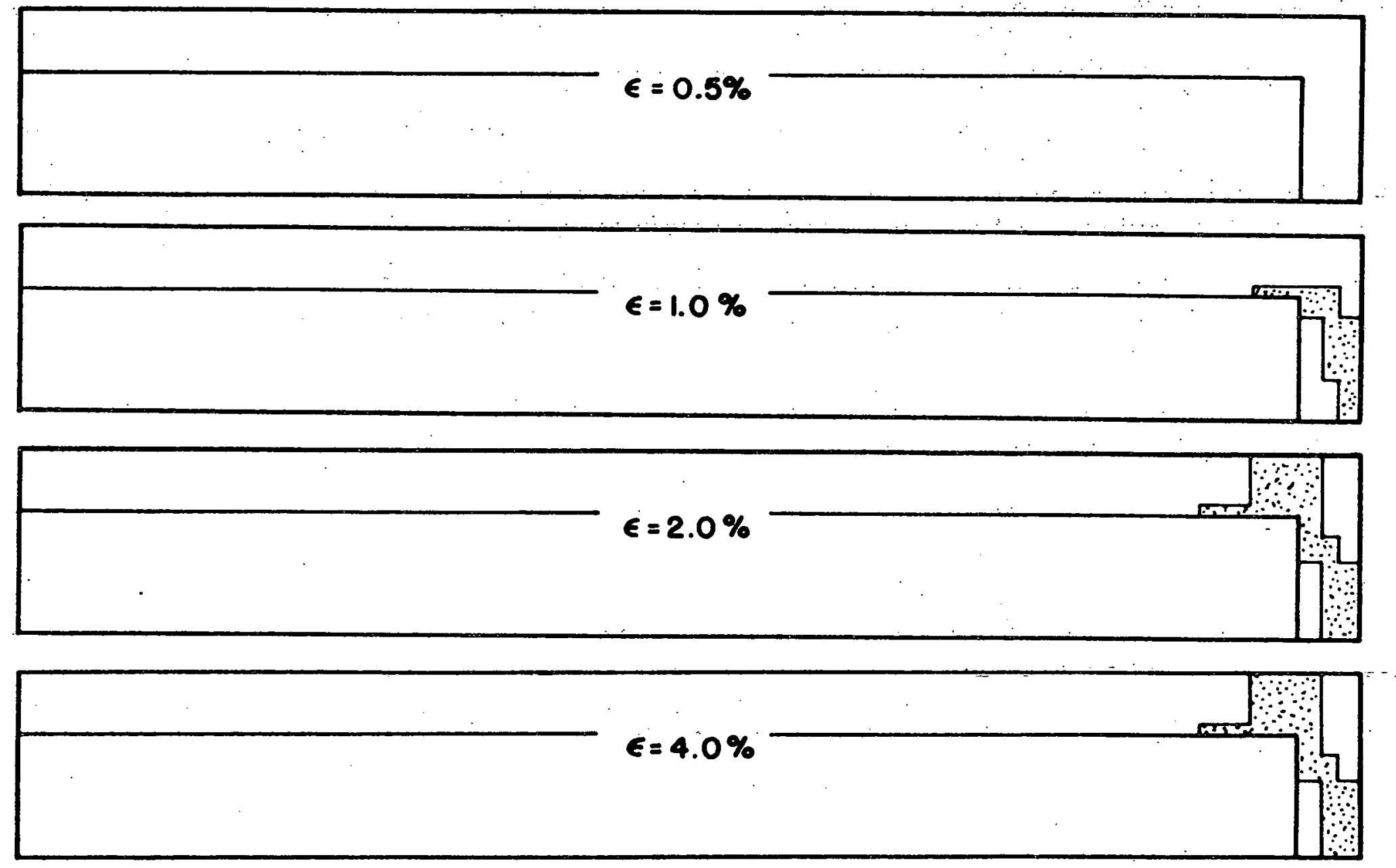

Fig. 70. Yielding of Matrix at Different Composite Stroins $\left(r_{2} / r_{3}=0.67\right.$, Matrix Yield Strain $=0.5 \%$, Axial Strain Imposed on One Boundary) 
given previously for the matrix yield stress of 8,000 psi apply also for the yield stress of 20,000 psi. Comparison of Fig. 70 with Fig. 65 shows that the growth of yield zone is also identical with that in the case of matrix yield stress of 8,000 psi.

The stress distributions for $r_{2} / r_{1}=0.25$ and the matrix yield stress of 8,000 psi are shown in Figs. 71-74. Yielding of the matrix at different strain levels is shown in Fig. 75. The average composite stresses are given in Table 8.

Table 8. Average Composite Stresses for $r_{2} / r_{1}=0.25$ and $\left(\sigma_{y}\right)_{m}=8,000$ psi

\begin{tabular}{llll}
\hline $\begin{array}{c}\text { Composite Axial } \\
\text { Strain }\end{array}$ & $\begin{array}{c}\text { Matrix Yield } \\
\text { Strain }\end{array}$ & $\begin{array}{c}\text { Matrix Yield } \\
\text { Stress }\end{array}$ & $\begin{array}{c}\text { Average Composite } \\
\text { Stress }\end{array}$ \\
\hline 0.5 percent & 2.0 percent & $8,000 \mathrm{psi}$ & $4,410 \mathrm{psi}$ \\
1.0 percent & 2.0 percent & $8,000 \mathrm{psi}$ & $8,350 \mathrm{psi}$ \\
2.0 percent & 2.0 percent & $8,000 \mathrm{psi}$ & $12,670 \mathrm{psi}$ \\
3.0 percent & 2.0 percent & 8,000 psi & $13,930 \mathrm{psi}$ \\
\hline
\end{tabular}

Axial stresses in the fiber (Fig. 7l) attain the maximum value in less than thirteen fiber diameters from the fiber end. The maximum stress in the fiber increases with the composite strain. The change in maximum fiber stress as the composite strain is increased from 2.0 to 3.0 percent is small because of the extensive yielding of the matrix at these strain levels (Fig. 75). A linear increase in the fiber stress near the end is due to the yielding of the matrix at the interface.

Shear stresses at the interface are shown varying along the fiber length in Fig. 72. Shear stresses near the fiber end are high. For the composite strain of 0.5 percent, the shear stress drops very rapidly and becomes zero in ten fiber diameters away from the end. For composite strain of 1.0 percent the shear stress remains constant for some distance, which is even longer for strains of 2.0 and 


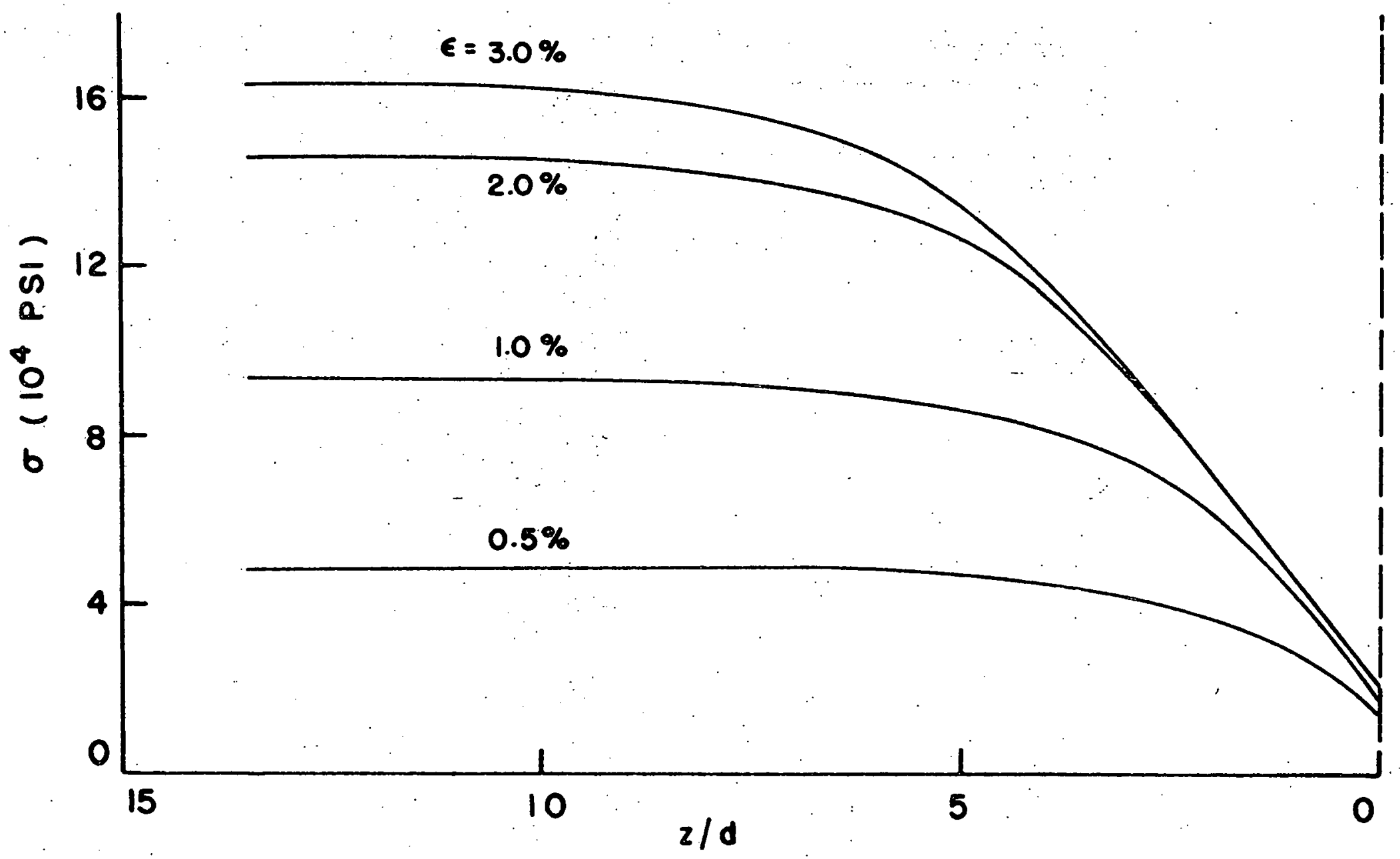

Fig. 71. Fiber Axial Stresses Along Fiber Axis $\left(r_{2} / r_{1}=0.25\right.$, Matrix

Yield Strain $=2.0 \%$, Axial Strain Imposed on One Boundary) 


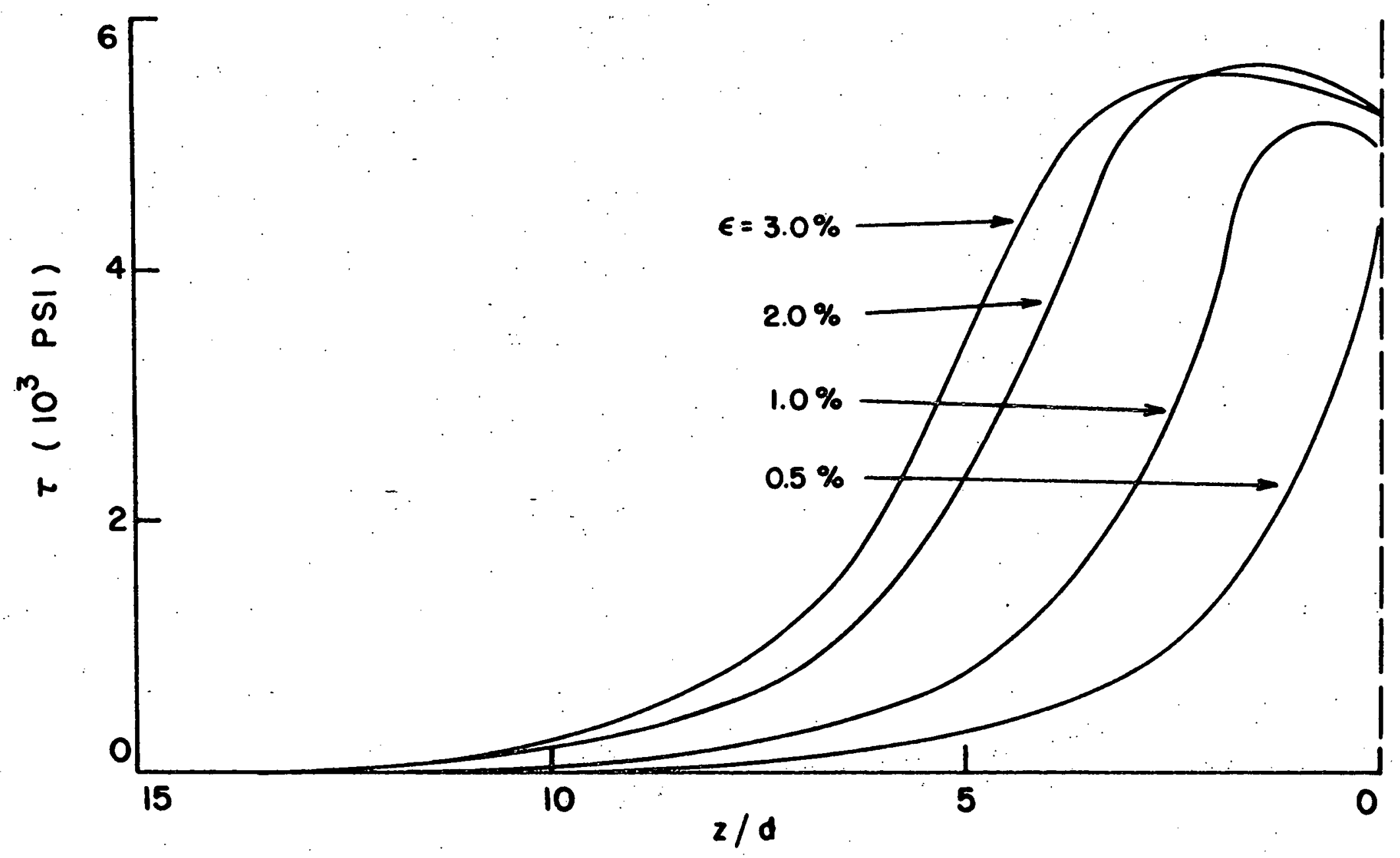

Fig. 72. Interfacial Shear Stresses Along Fiber Axis $\left(r_{2} / r_{1}=0.25\right.$, Matrix Yield Strain $=2.0 \%$, Axial Strain Imposed on One Boundary) 


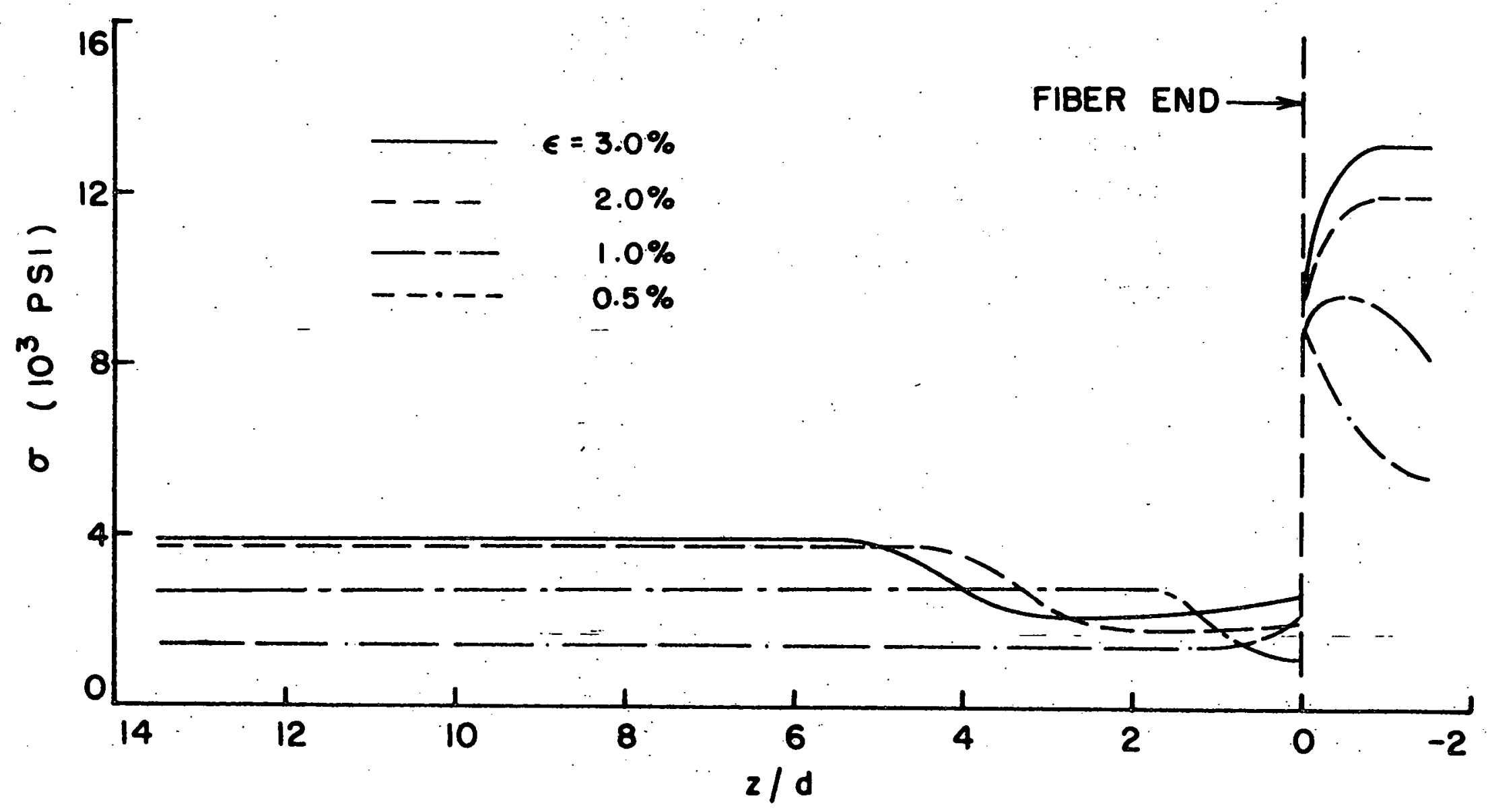

Fig. 73. Matrix Axial Stresses Along Fiber Axis $\left(r_{2} / r_{j}=0.25\right.$, Matrix Yield Strain. $=2.0 \%$, Axial Strain Imposed on One Boundary) 


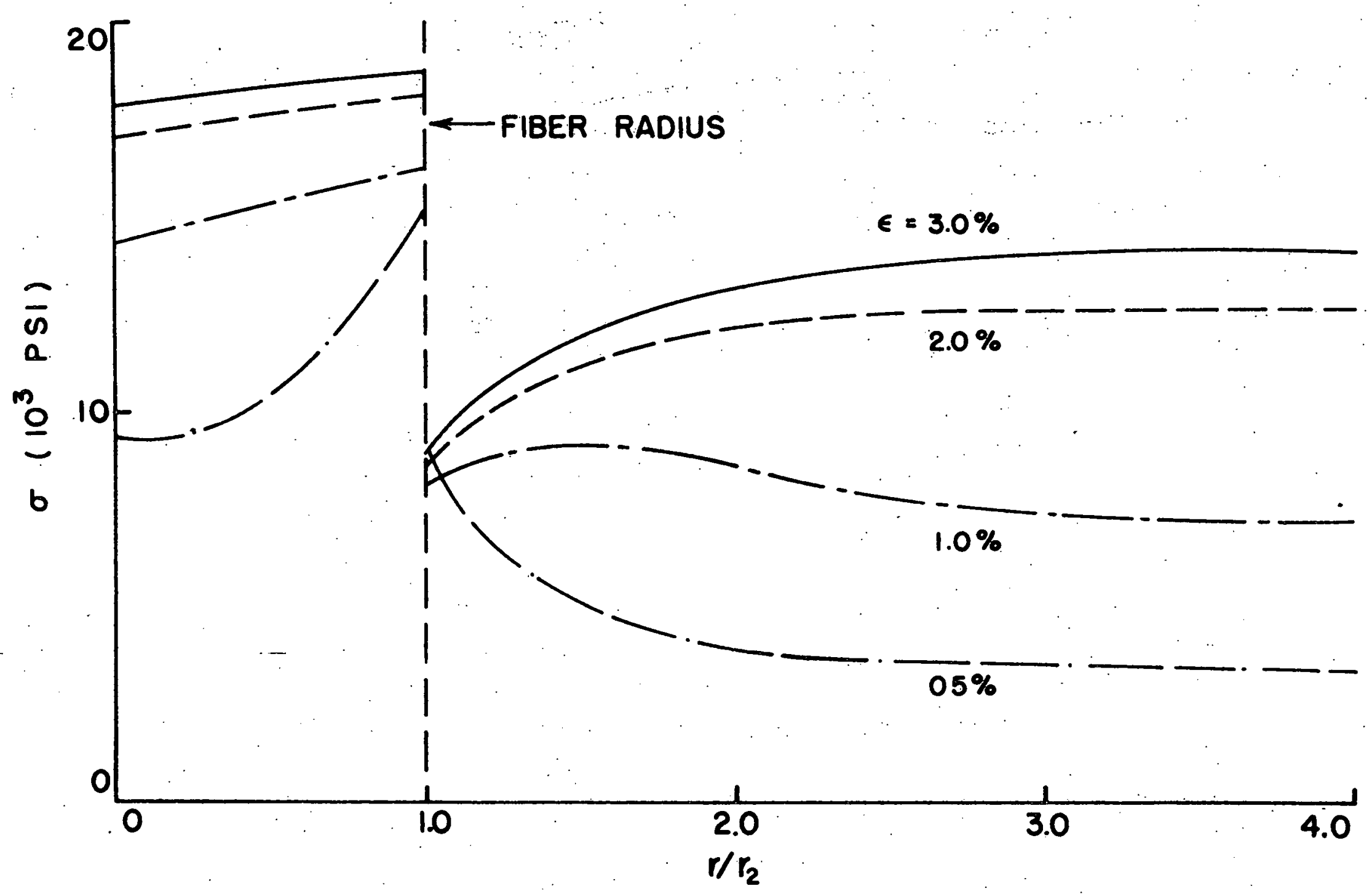

Fig. 74. Radial Variation of Matrix Axial Stresses Near Fiber End $\left(r_{2} / r_{1}=0.25\right.$, Matrix Yield Strain $=2.0 \%$, Axial Strain Imposed on One Boundary) 

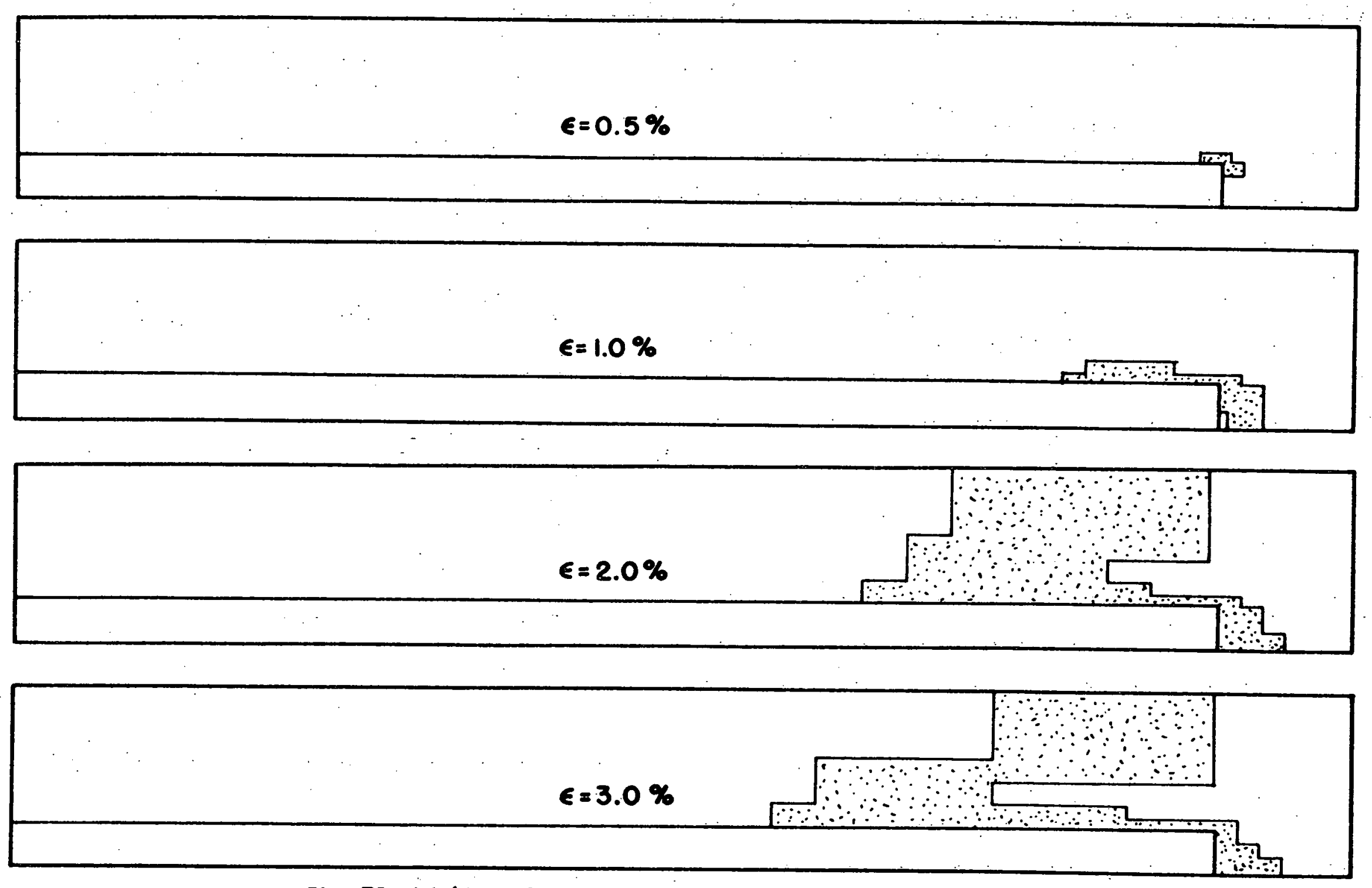

Fig. 75. Yielding of Matrix at Different Composite Strains $\left(r_{2} / r_{1}=0.25\right.$, Matrix

Yield Strain $=2.0 \%$, Axial Strain Imposed on One Boundary) 
3.0 percent, and then drops rapdily. The stress in all the cases reduces to zero within thirteen fiber diameters which is consistent with the observations bosed on Fig. 71. The matrix axial stresses at the interface are plotted as a function of distance from the fiber end in Fig. 73. Variation in the radial direction of the matrix axial stress (in the elements adjacent to the fiber end, refer Fig. 42) is shown in Fig. 74. Both Fig. 73 and 74 show discontinuity in the matrix stress near the physical discontinuity at the fiber end. Yielding of the matrix is shown in Fig. 75. At 0.5 percent composite strain only a few elements of the matrix have yielded near the fiber end. As the composite strain is raised to 1.0 percent more matrix yields near the fiber end and also along the interface. There is an extensive yielding of the matrix at the composite strains of 2.0 and 3.0 percent.

Stress-Strain Curves With Axial Strains Imposed on One Boundary of the Representative Cell

Predicted stress-strain curves for the composite with the fiber packing of Fig. 38 are shown in Figs. 76 and 77. Effect of change in fiber contents is shown in Fig. 76. Initial modulus of elasticity of the composite with 5.62 percent fibers is $0.88 \times 10^{6}$ psi compared to $3.58 \times 10^{6}$ psi for the composite with 42.4 percent fibers. It was pointed out in Section IV that the Halpin and Tsai equation (29) may also be used to calculate the modulus of the composite with discontinuous fiber reinforcement. In Section IV, the elastic modulus calculated by this equation for 42.4 percent fiber contents compared favorably with the present predictions. The Halpin and Tsai equation predicts an elastic modulu's of $0.83 \times 10^{6}$ psi for 5.62 percent fiber contents and a fiber aspect ratio of 27 (i.e. for the present fiber geometry). This value is also quite comparable with the prediction of the present study. Fig. 76 shows that the composite with 5.62 percent fibers has low initial modulus $\left(0.88 \times 10^{6}\right.$ psi), but it shows the effect of local yielding 


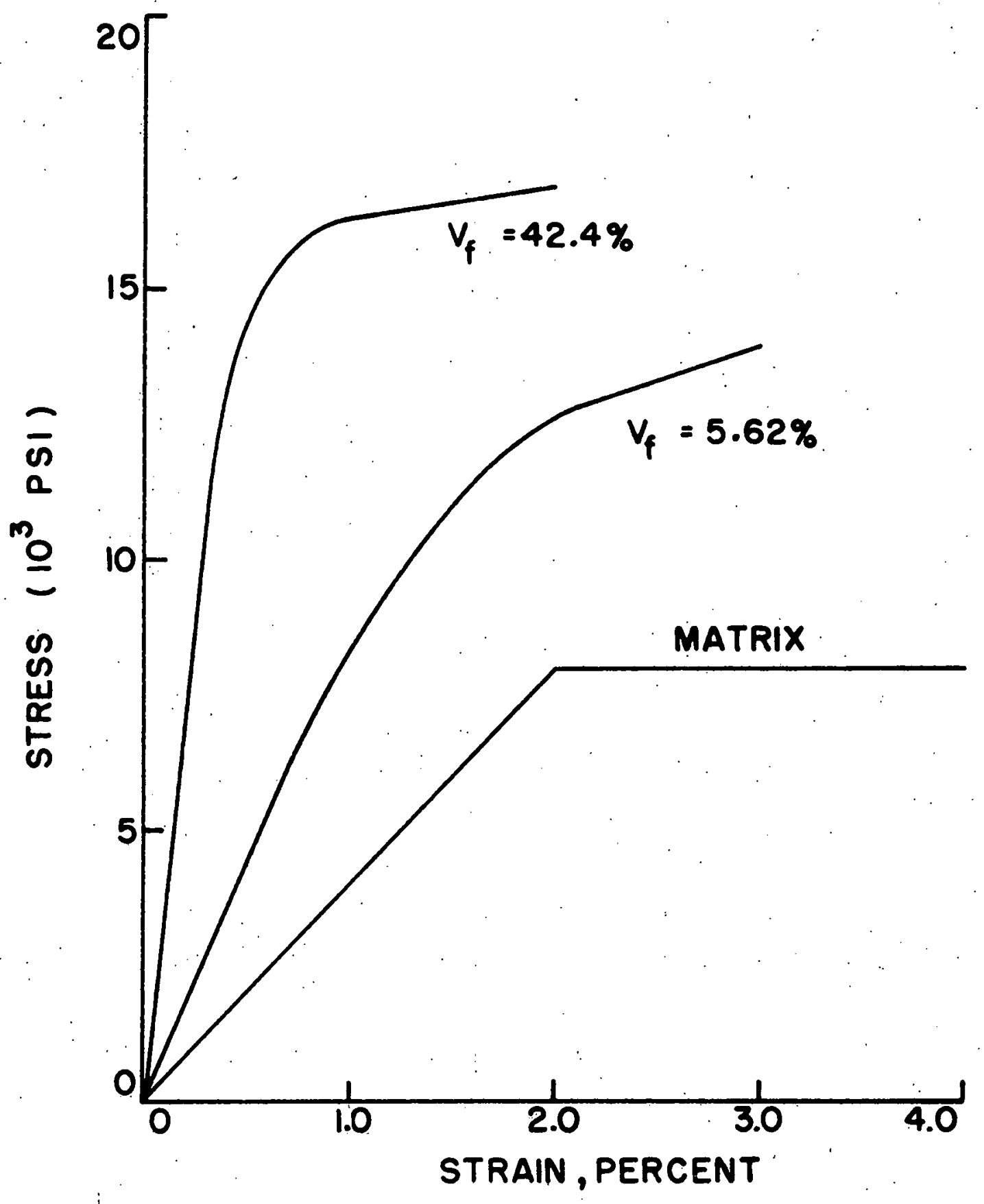

Fig. 76. Stress-Strain Curves for Matrix and Composites with Different Fiber Volume Fractions 
122

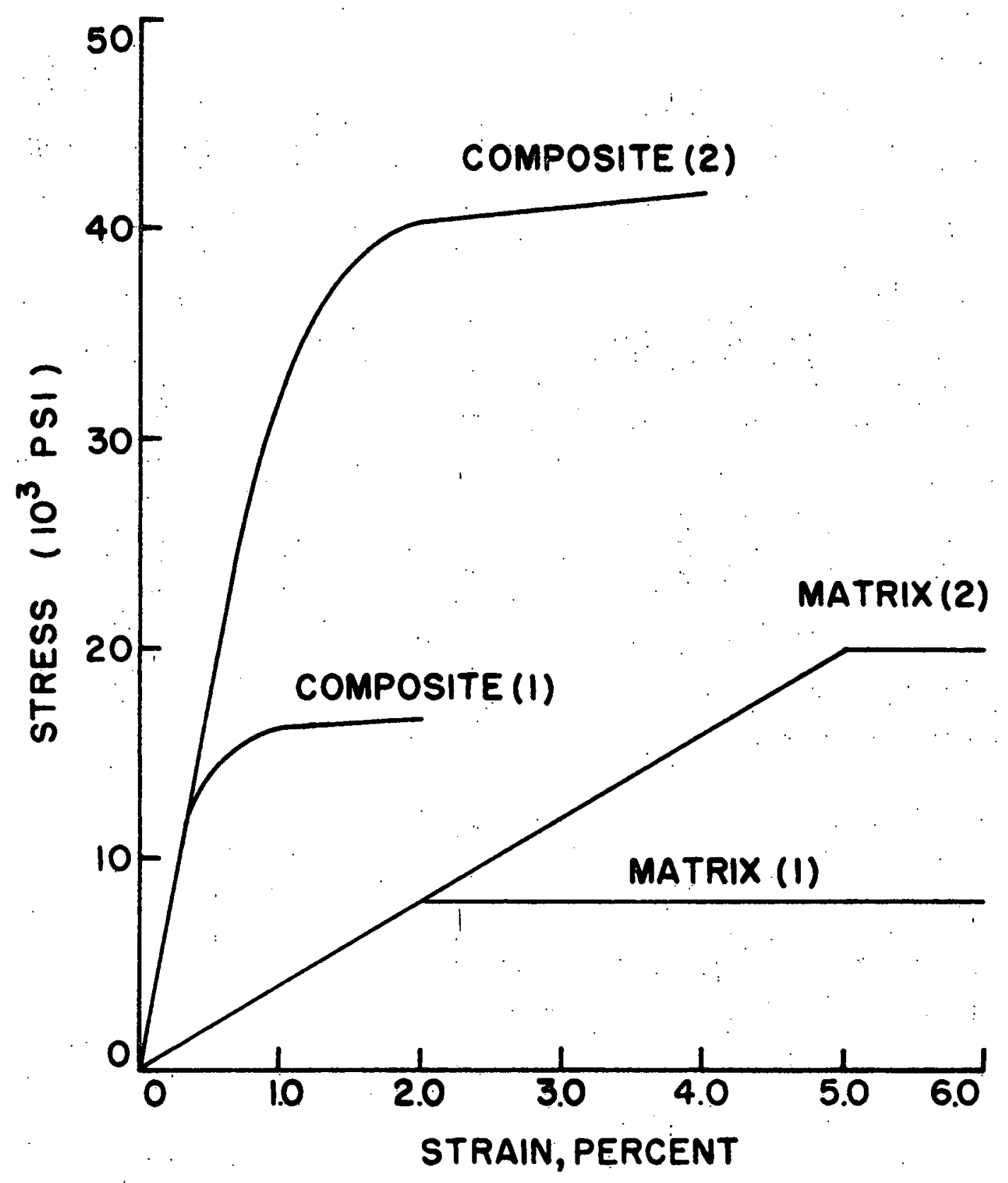

Fig. 77. Stress-Strain Curves for Matrices and Corresponding Composites 
of matrix after 1.0 percent composite strain. Initial modulus of the composite with 42.4 percent fibers is quite high $\left(3.58 \times 10^{6}\right.$ psi) but this composite shows yielding even at 0.5 percent composite strain. The effect of a change in the matrix yield stress is shown in Fig. 77 . It is quite clear that the matrix yield stress does not influence the initial modulus of the composite. Yield stress of the composite increases in about the same proportion as the yield stress of the matrix. However, in both cases the composite yield strain is much smaller than the matrix yield strain. This is because the fiber end produces high strain concentrations in the matrix in its vicinity which cause yielding of the matrix between the two rows of fibers. The stresses do not build up beyond a certain value although the strains are large. It was pointed out in an earlier section that the boundary conditions may be imposed in such a way that the average composite stress is not completely controlled by a weak section. Results of the analysis with such boundary conditions are presented in the next section.

Stresses and Stress-Strain Curve with Axial Strains Imposed on Entire Outer Boundary of the Representative Cell

Results of the analysis of the composites with the fiber packing shown in Fig. 59 are discussed in this section. The two geometries of the fibers (Fig. 60) used were the same as in the previous case. The component properties were also assumed to be the same as in the preceding section of this chapter. However, the analysis was not carried out for the matrix yield stress of 20,000 psi because of the similarity of results with the yield stress of 8,000 psi.

The stress distributions for $r_{2} / r_{1}=0.67$ are shown in Figs. 78-81. Yielding of the matrix at different strain levels is shown in Fig. 82. The average composite stresses given in Table 9 were calculated by taking an average of the axial stresses over the entire volume of the composite. In the previous case the average over the 


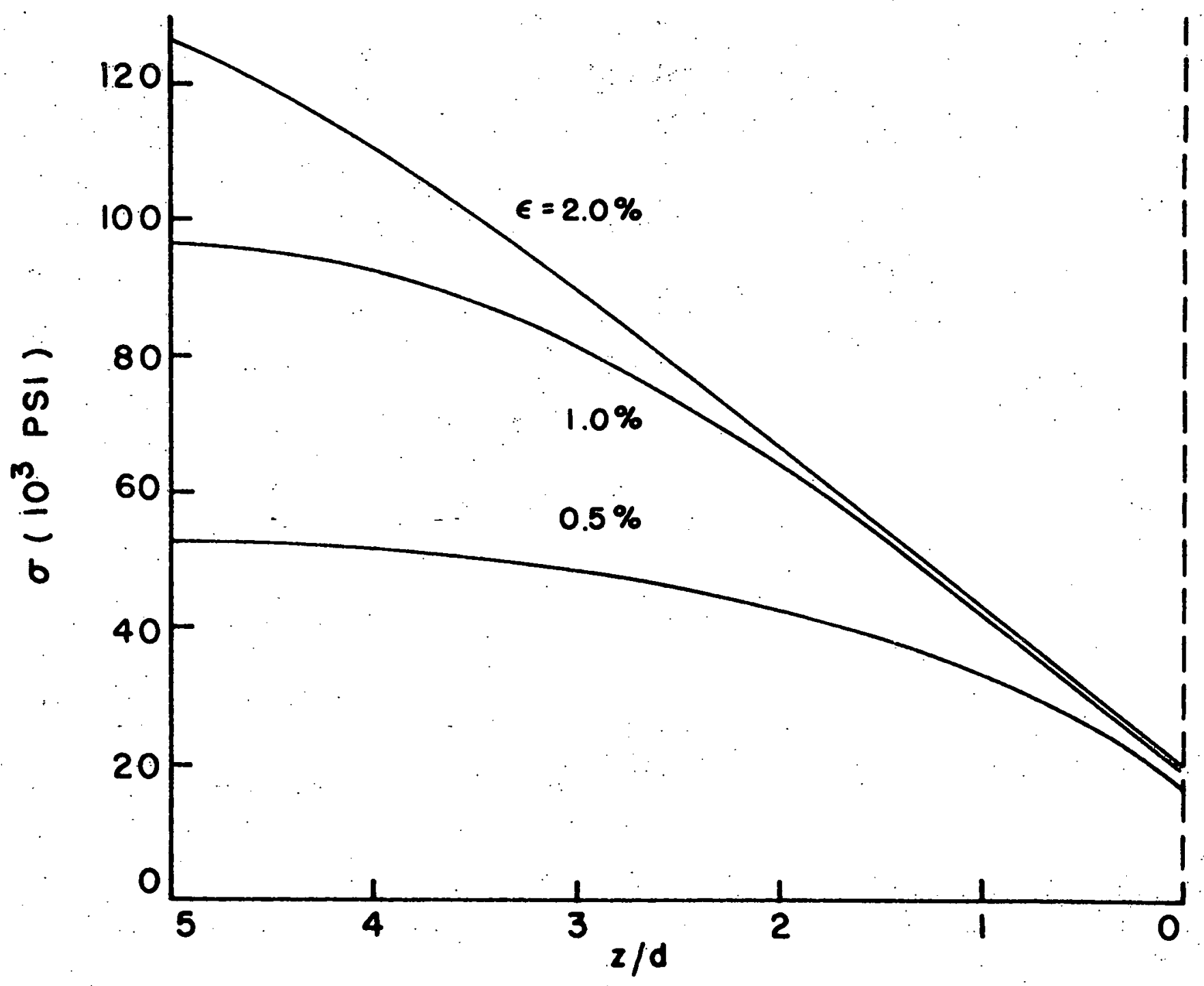

Fig. 78. Fiber Axial Stresses Along Fiber Axis $\left(r_{2} / r_{1}=0.67\right.$, Matrix Yield Strain $=2.0 \%$, Axial Strain Imposed on Entire Outer 


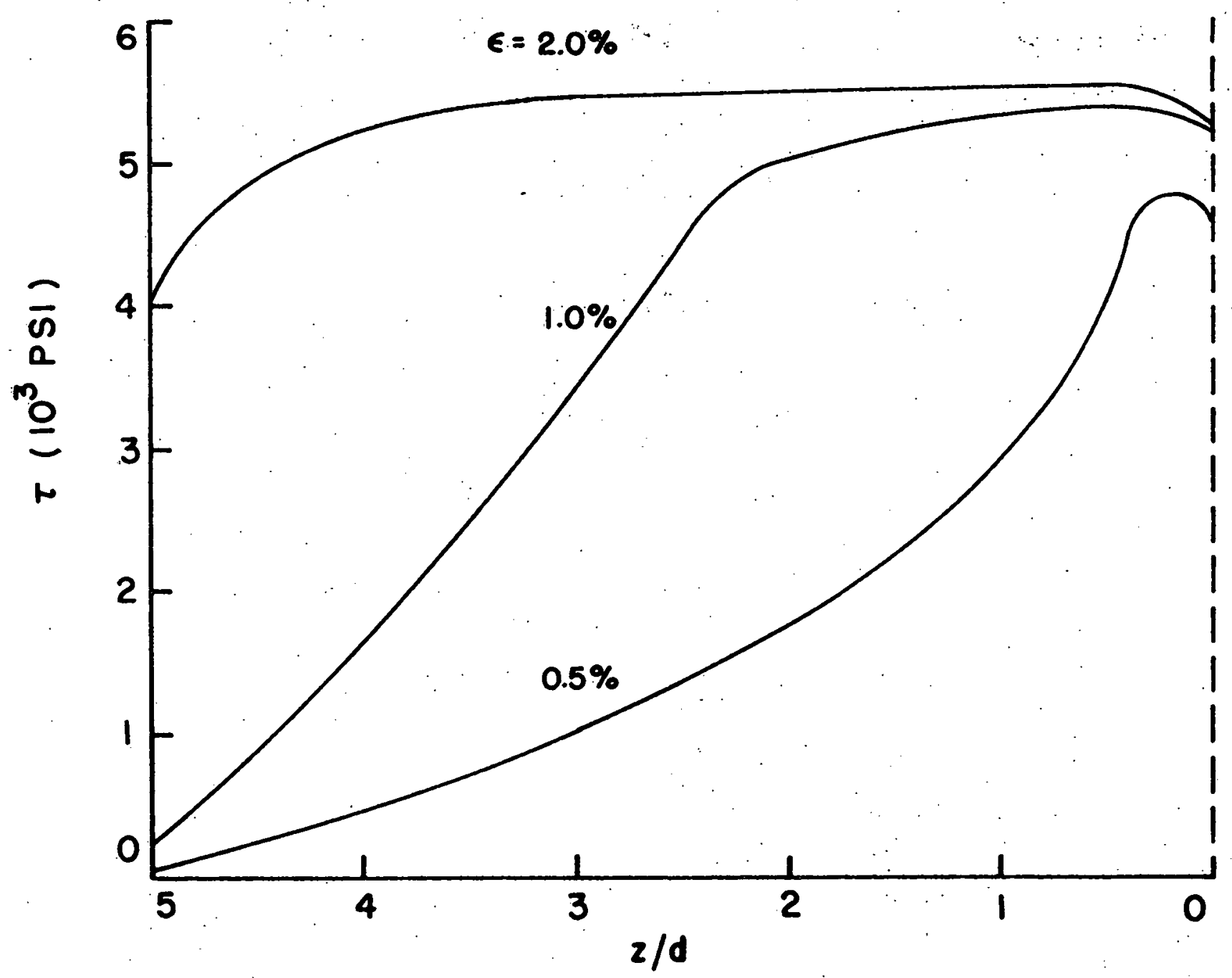

Fig. 79: Interfacial Shear Stresses Along Fiber Axis $\left(r_{2} / r_{1}=0.67\right.$, Matrix Yield Strain $=2.0 \%$, Axial Strain Imposed on Entire Outer Boundary) 


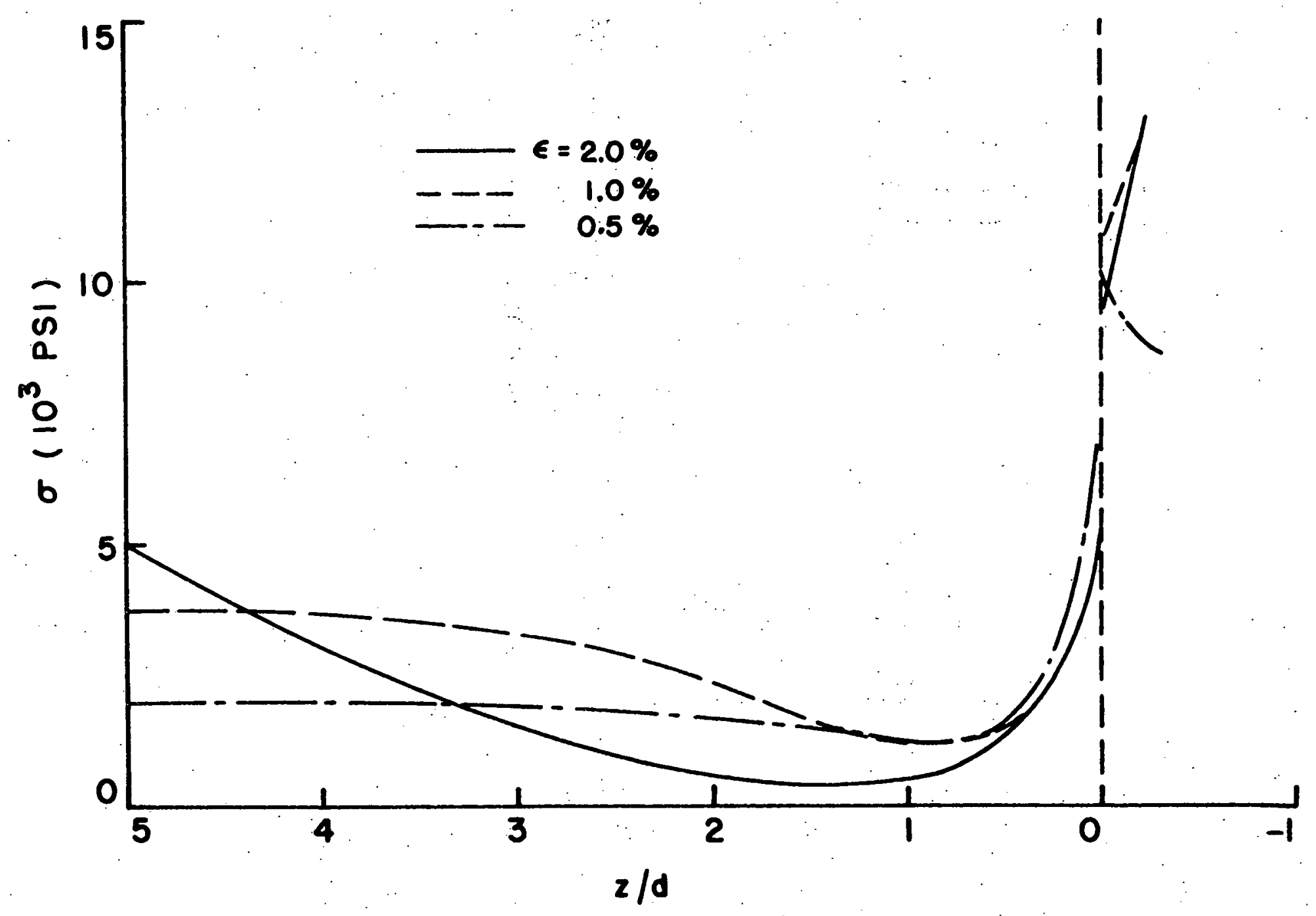

Fig. 80. Matrix Axial Stresses Along Fiber Axis $\left(r_{2} / r_{1}=0.67\right.$, Matrix Yield Strain $=2.0 \%$, Axial Strain Imposed on Entire Outer

Boundary) 


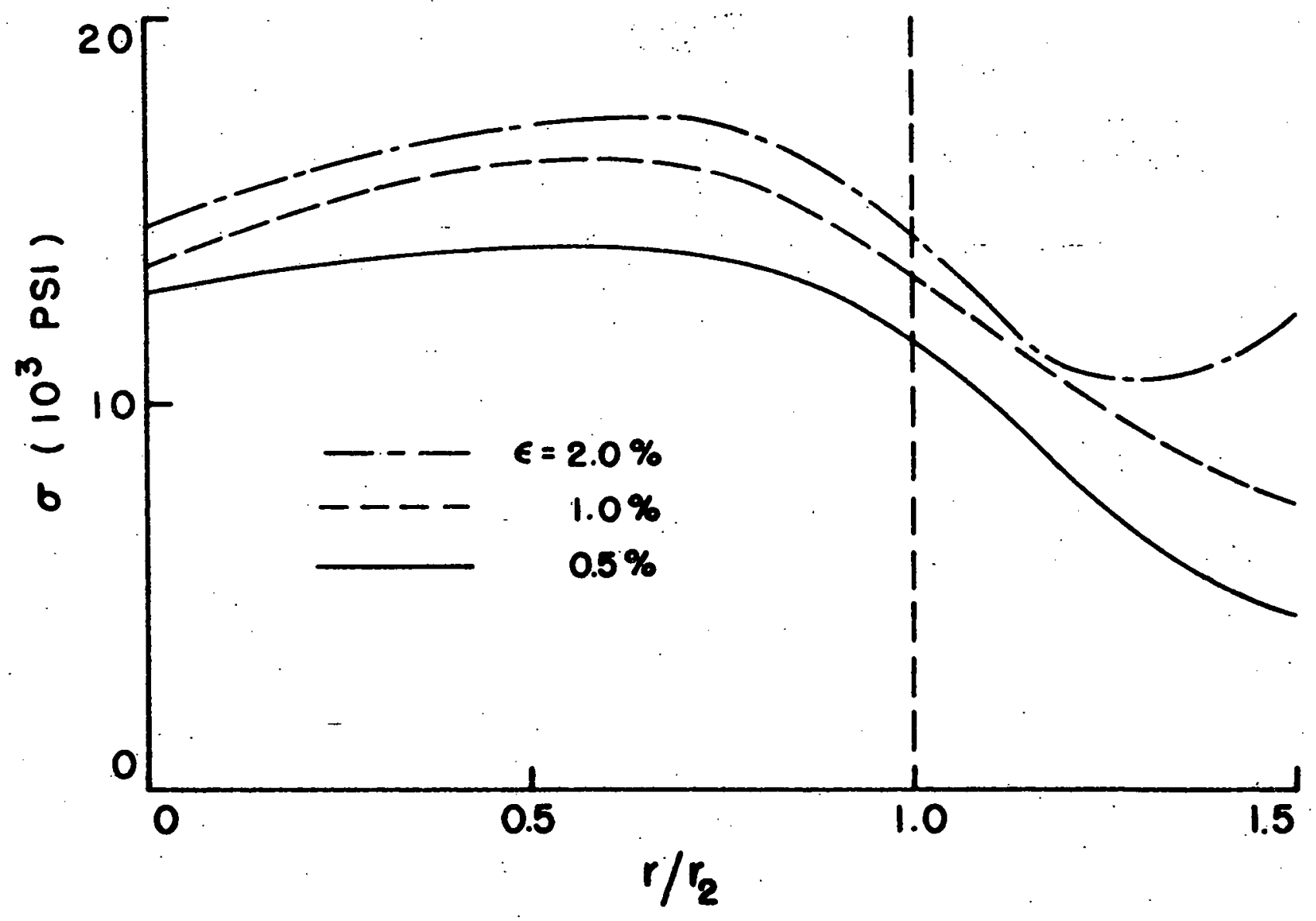

Fig. 81. Radial Variation of Matrix Axial Stresses Near Fiber End $\left(r_{2} / r_{1}=0.67\right.$, Matrix Yield Strain $=2.0 \%$, Axial Strain Imposed on Entire Outer Boundary) 


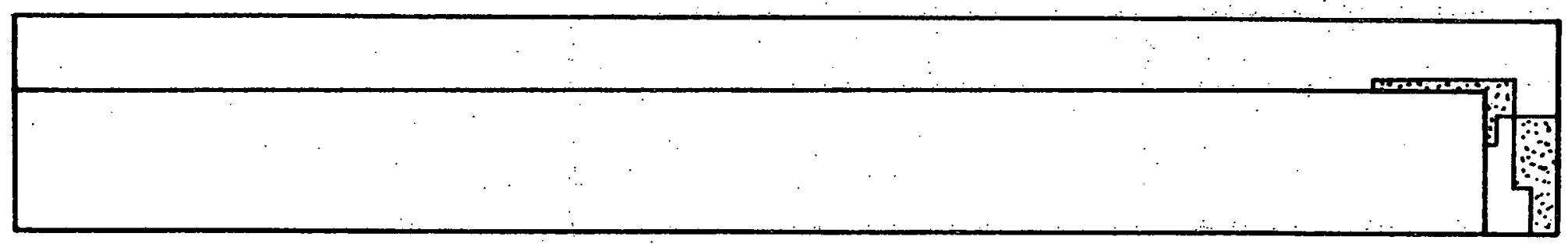

$\epsilon=0.5 \%$

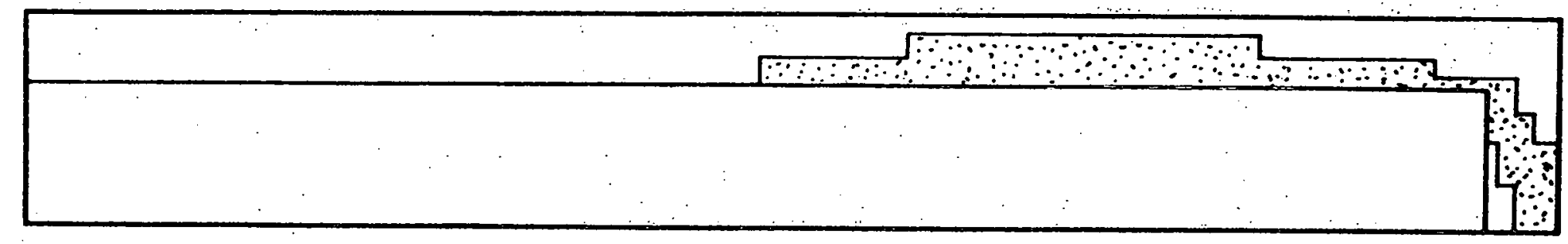

$\epsilon=1.0 \%$

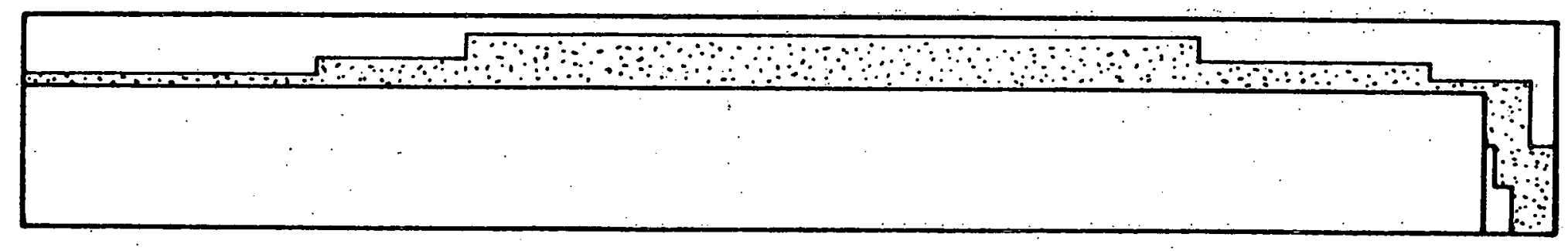

$\epsilon=2.0 \%$

Fig. 82. Yielding of Marrix at Different Composite Strains $\left(r_{2} / r_{1}=0.67\right.$, Matrix Yield Strain $=2.0 \%$, Axial Strain Imposed on Entire Outer Boundary) 
entire volume was not necessary because the average stress on any cross-rection was the same since the shear stress on the outer boundary of the representative cell (Fig. 39) was prescribed to be zero. But with the present boundary conditions shear stresses exist on the outer boundary of the representative cell and hence the average axial stresses on different cross-sections are different.

Table 9. A verage Composite Stresses for $\frac{r_{2}}{r_{1}}=0.67$ and $\left(\sigma_{y}\right)_{m}=8,000$ psi

\begin{tabular}{|c|c|c|c|}
\hline $\begin{array}{l}\text { Composite Axial } \\
\text { Strain }\end{array}$ & $\begin{array}{l}\text { Matrix Yield } \\
\text { Strain }\end{array}$ & $\begin{array}{l}\text { Matrix Yield } \\
\text { Stress }\end{array}$ & $\begin{array}{c}\text { Average Composite } \\
\text { Stress }\end{array}$ \\
\hline 0.5 percent & 2.0 percent & 8,000 psi & 19,670 psi \\
\hline 1.0 percent & 2.0 percent & 8,000 psi & 31,440 psi \\
\hline 2.0 percent & 2.0 percent & 8,000 psi & 35,500 psi \\
\hline
\end{tabular}

The axial stresses in fibers are shown in Fig. 78 for the composite strain of $0.5,1.0$ and 2.0 percent. The stresses near the fiber end are low but increase away from the end in all cases. At 0.5 percent composite strain the fiber stress reaches a constant value within five fiber diameters from the end. However, unlike the previous case (Fig. 61), the fiber stresses at the composite strains of 1.0 and 2.0 percent do not attain a constant value in the assumed fiber length (i.e., 10.375 fiber diameters). This is because the redistribution of load along the fiber length allows higher stresses to be carried by the fibers at higher composite strains. Longer fibers are needed to transfer this higher stress. The transfer of load is aided by the yielding of the matrix along the fiber-matrix interface as shown in Fig. 82. A linear increase in the fiber axial stress at 2.0 percent composite strain is, once again, due to the yielding of the matrix at the interface. 
The shear stresses at the interface are shown varying along the fiber length in Fig. 79. The stresses are high near the fiber end at all the composite strains (0.5, 1.0 and 2.0 percent). At 0.5 percent composite strain, the interfacial shear stress starts decreasing rapidly very near the fiber end, whereas due to yielding of the matrix it remains nearly constant for more than two fiber diameters at 1.0 percent composite strain and for more than four fiber diameters at 2.0 percent composite strain. The stress distributions in Fig. 78 are consistent with the ones in Fig. 79 according to equation ( 7 ) in Section IV. The matrix axial stresses of the interface are plotted as a function of distance from the fiber end in Fig. 80 . The stresses increase sharply near the fiber end and show a discontinuity due to the discontinuity in the material properties at this point. Variation in the radial direction of the matrix axial stresses (in the elements adjacent to the fiber end, refer Fig. 42) is shown in Fig. 81. The variation in the stresses is not very large due to the yielding of the matrix near the end. Fig. 82 shows that only few elements of the matrix near the fiber end have yielded at 0.5 percent composite strain. The yielded region of the matrix enlarges as the composite strain increases. There is extensive yielding of the matrix at 2.0 percent composite strain.

The predicted stress-strain curve for the composite with the fiber packing of Fig. 59 is shown in Fig. 83. The stress-strain curve in Fig. 83 shows much higher values of stresses compared to the corresponding curve in Fig. 77. This is not unexpected because the higher stresses are expected due to the load redistribution between the fibers. There are no experimental data in the literature to the author's knowledge which could be directly compared with the present predictions. Studies of Kelly and Tyson ${ }^{(33)}$ concerning the tensile properties of fiber-reinforced metals show that in the case of discontinuous molybdenum fibers in copper matrix (with yield stress $\approx 10,000$ psi) the composite with 42 percent fibers has a yield stress 


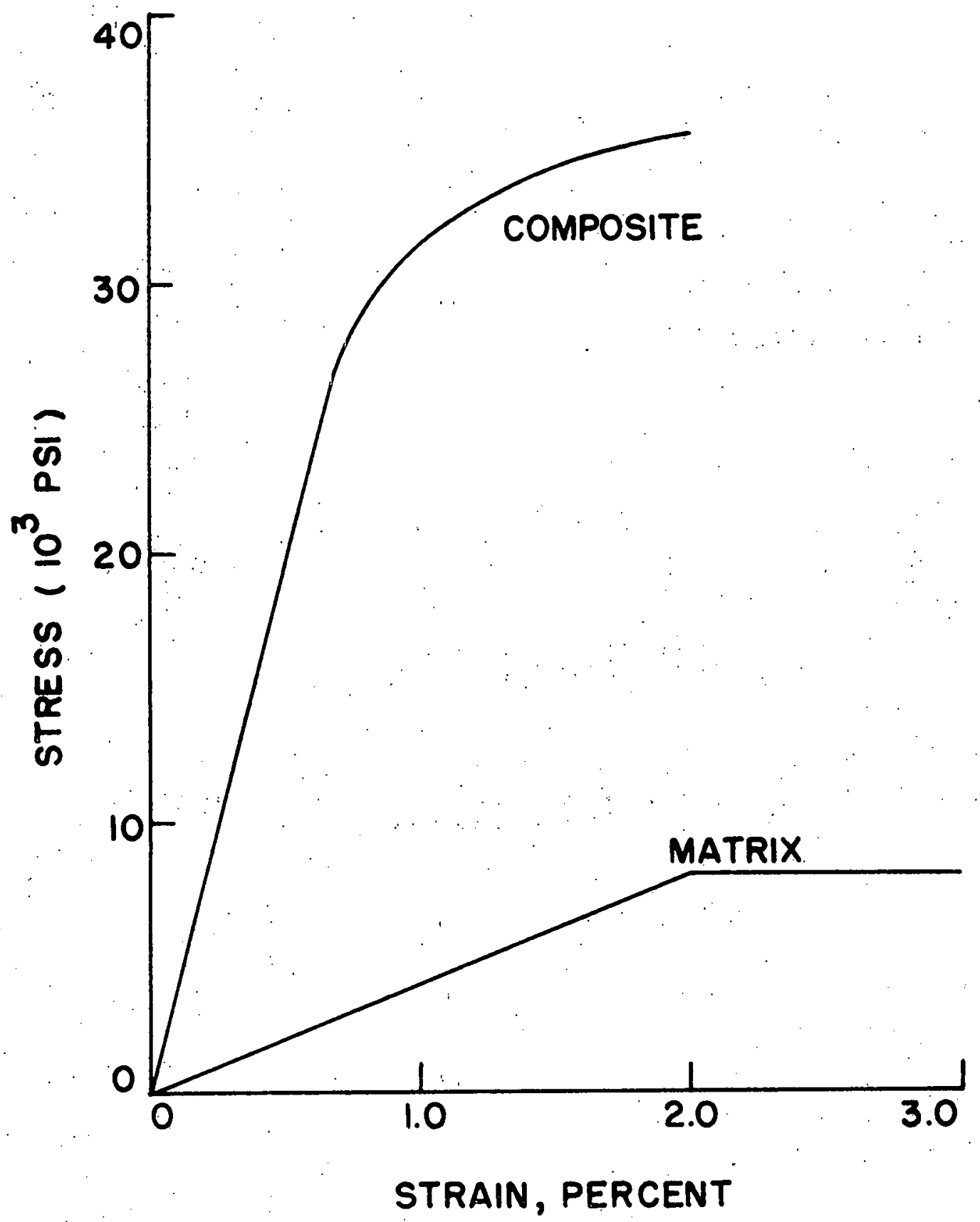

Fig. 83, Stress-Strain Curves for Matrix and Composite with Axial Strain Imposed on Entire Outer Boundary $\left(r_{2} / r_{1}=0.67\right)$ 
of about 40,000 psi. Studies of Weeton and Signorelli ${ }^{(34)}$ indicate that the composite reinforced with continuous $5 \mathrm{mil}$ diameter tungsten wire $\left(v_{f}=53.6\right.$ percent) has a yield stress of about 150,000 psi compared to 10,000 psi yield stress of the copper matrix. In the light of these studies, the stress-strain curve shown in Fig. 83 seems closer to the reality. Definite conclusions can be drawn only after more experimental data are accumulated and the analyses are carried out by varying other parameters such as volume fraction of fibers and fiber aspect ratio.

For the $r_{2} / r_{1}=0.25$ and fiber aspect ratio of 27 , the stress distributions were obtained for the composite strains of 1.0 and 2.0 percent. The results are shown in Figs. 84-87. Yielding of the matrix at the two strain levels is shown in Fig. 88. These results are quite similar to the ones obtained for the closer fiber packing $\left(r_{2} / r_{1}=0.67\right)$. It may be noted that at the low volume fraction of fibers (i.e.., 5.62 percent) the fiber aspect ratio of even 27 is not enough for the fiber axial stress to reach a constant value. It suggests that the analyses with higher fiber aspect ratios would be of considerable interest.

The present studies indicate that the behaviour of a composite can be simulated very closely by carefully imposing the boundary conditions on a representative cell. The studies show the effect of yielding of the matrix on the internal stresses and predict the stress-strain relation of the composite. The influence of changing the matrix yield stress has also been studied. However, the studies are not extensive enough in some areas of the composite materials. Studies have been carried out with only two volume fractions of the fibers (5.62 and 42.4 percent) and at each volume fraction only one fiber aspect ratio has been studied. The effect of relative elastic properties of the fiber and the matrix has not been investigated. For the better understanding of the behavior of the composite materials further investigations should be carried out in these areas. More attention should be devoted to the 


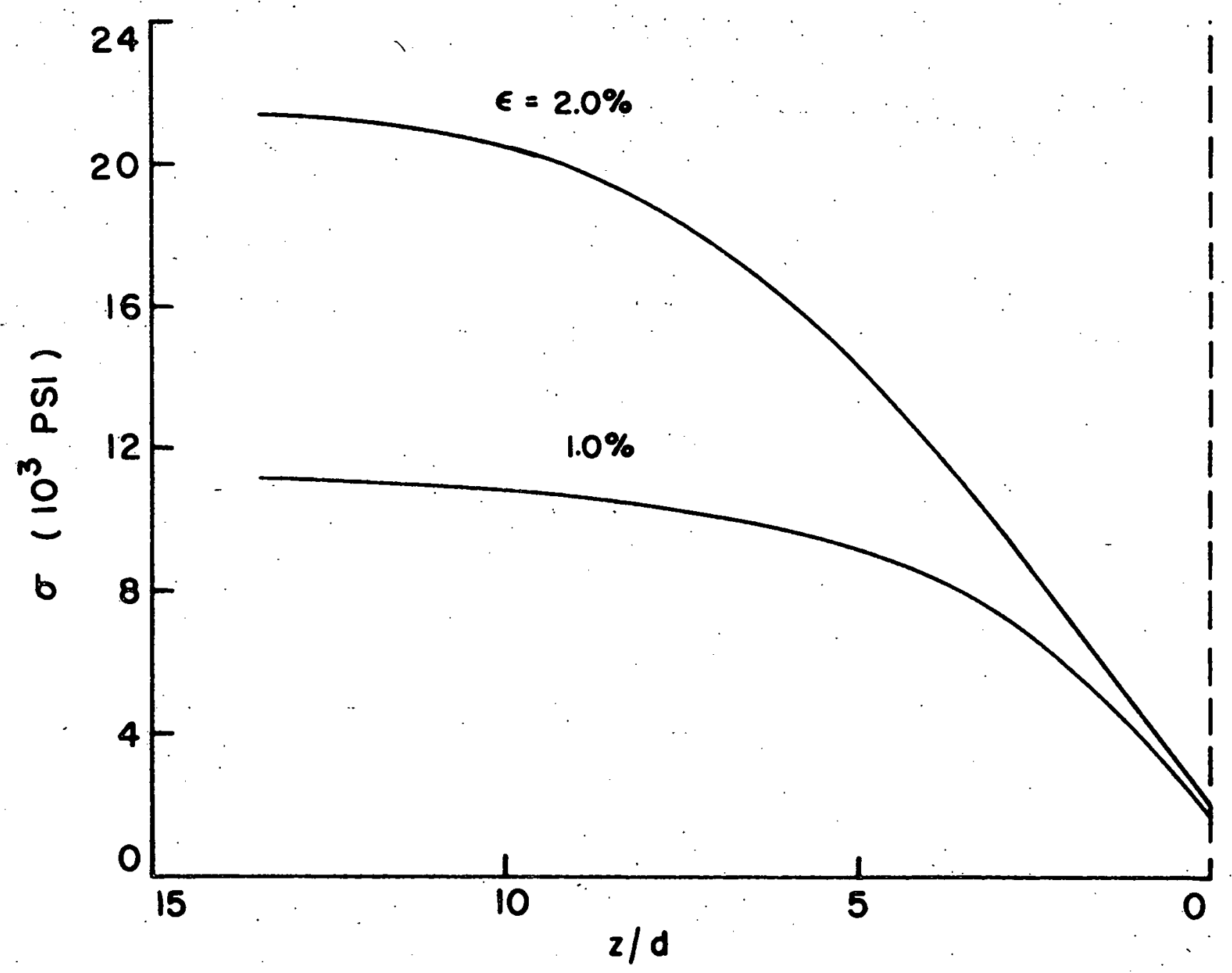

Fig. 84. Fiber Axial Stresses Along Fiber Axis $\left(r_{2} / r_{1}=0.25\right.$, Matrix Yield Strain $=2.0 \%$, Axial Strain Imposed on Entire Outer Boundary) 


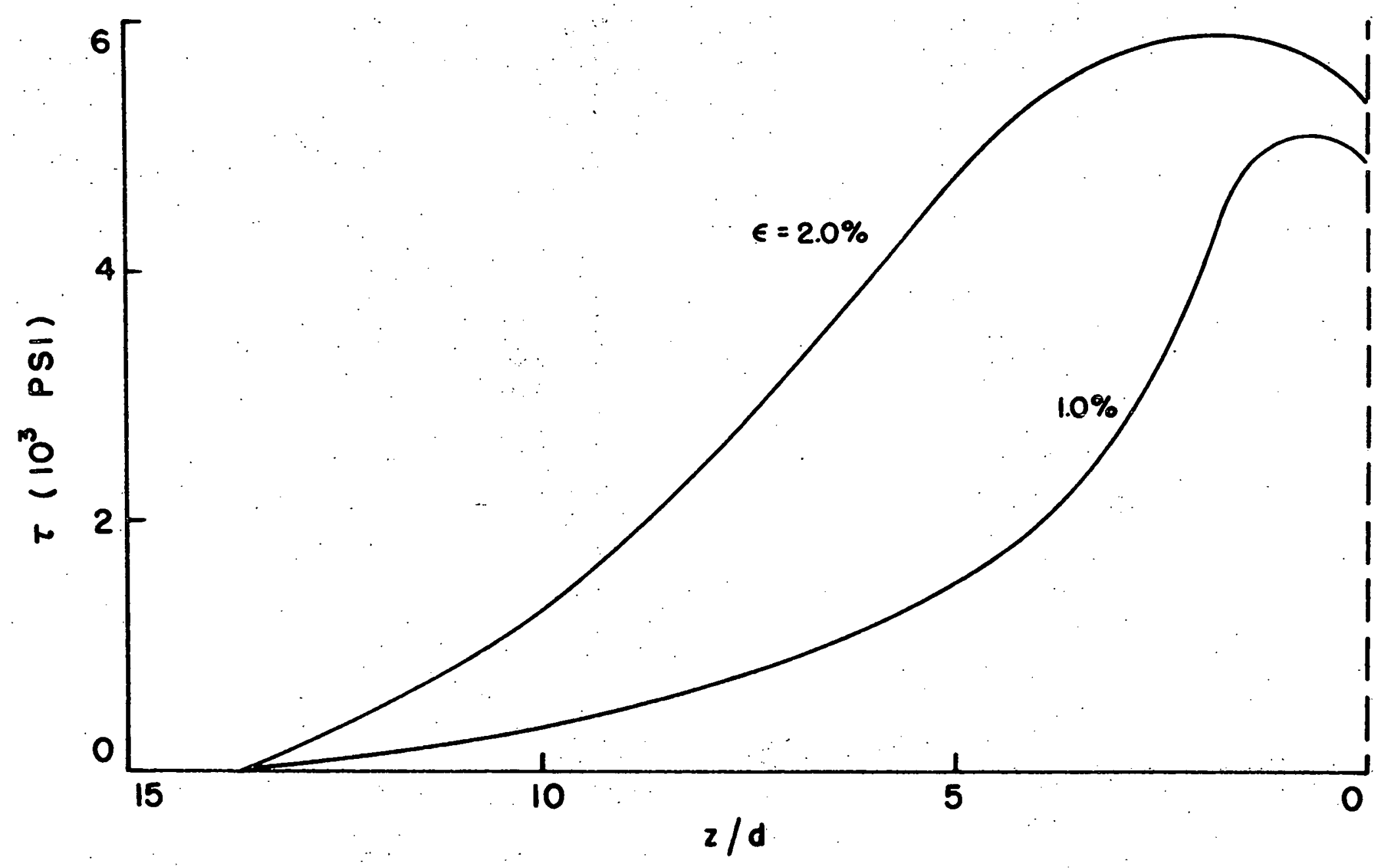

Fig. 85. Interfacial Shear Stresses Along Fiber Axis $\left(r_{2} / r_{1}=0.25\right.$, Matrix Yield Strain $=2.0 \%$, Axial Strain Imposed on Entire Outer Boundary) 


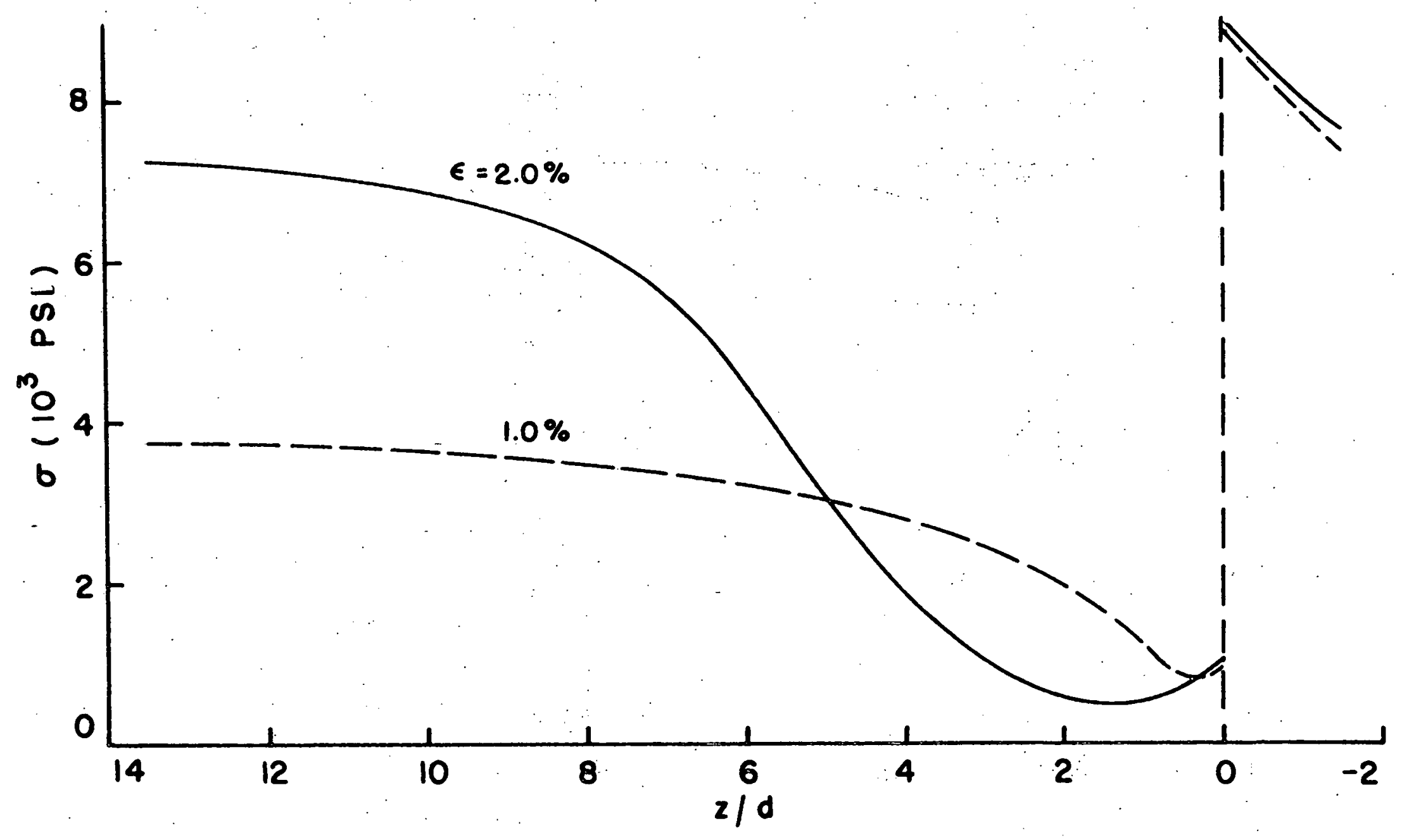

Fig. 86. Matrix Axial Stresses Along Fiber Axis $\left(r_{2} / r_{1}=0.25\right.$, Matrix Yield Strain $=2.0 \%$, Axial Strain Imposed on Entire Outer Boundary) 


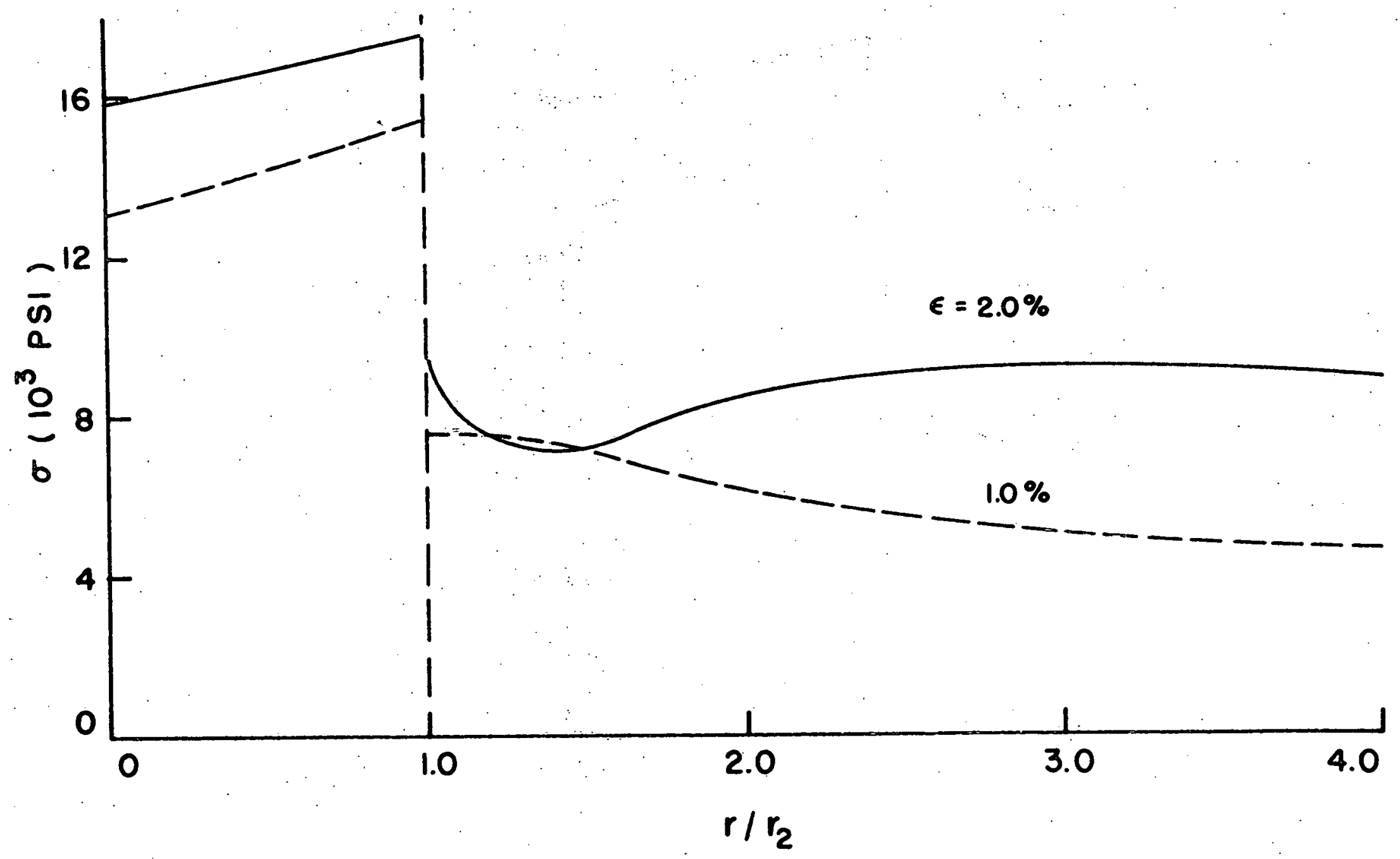

Fig. 87. Radial Variation of Matrix Axial Stresses Near Fiber End $\left(r_{2} / r_{1}=0.25\right.$, Matrix Yield Strain $=2.0 \%$, Axial Strain Imposed on Entire Outer Boundary) 


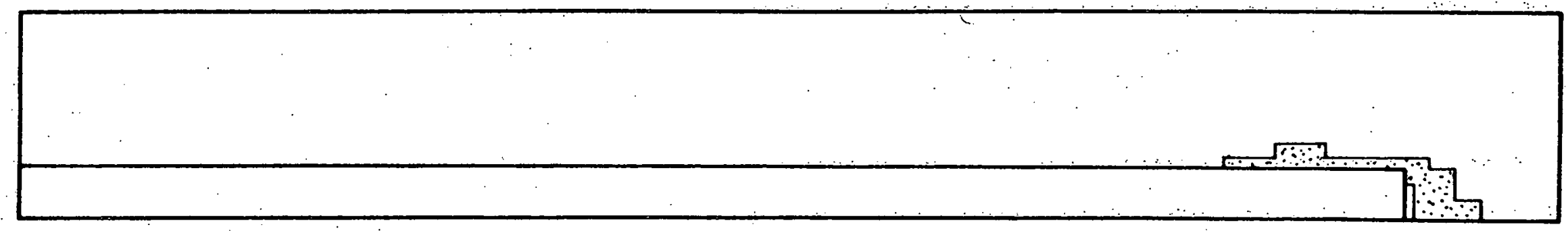

$\epsilon=1.0 \%$

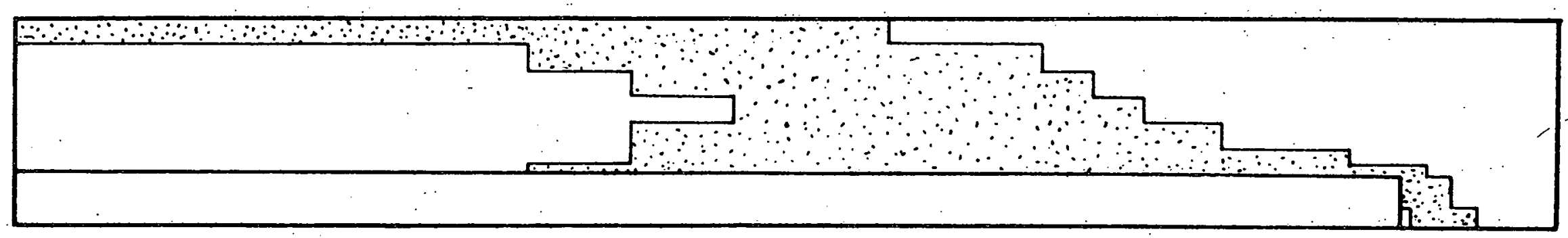

$\epsilon=2.0 \%$

Fig. 88. Yielding of Matrix at Different Composite Strains $\left(r_{2} / r_{1}\right)=0.25$, Matrix

Yield Strain $=2.0 \%$, Axial Strain Imposed on Entire Outer Boundary) 
selection of the model and the associated boundary conditions. Finally, to gain confidence regarding the usefulness of the analyses, experiments should be performed and the data compared. 


\section{CONCLUSIONS}

Finite element methods have been used to calculate internal stresses within a spherical particle or discontinuous fiber composite. The internal stresses have been analyzed as a function of interparticle spacing and the elastic constants have been calculated from a knowledge of these stresses. The effect of altering the interface properties has been simulated by assigning different properties to the finite elements forming the interface. For the case of composites with elasticplastic matrices the boundary conditions employed had to be altered in order to simulate the behavior of a real composite.

In the case of spherical particle composites, a comparison has been made between an axisymmetric analysis and a three-dimensional analysis to represent the geometrical arrangement of the particles. Although the axisymmetric analysis is easy to use and the computer running time is nominal, the axisymmetric cell used to represent the composite does not actually fill the entire space. Thus a three-dimensional analysis. was performed assuming the spheres were placed on the corners of a cube, to represent a simple cubic packing. For this case, the interparticle spacing can be directly related to the volume fraction of spherical particles in the composite. It is seen that the results of the two analyses are in good agreement. At low volume fractions or at great interparticle spacings the results are in agreement with the predictions of the Goodier theory for stresses around a spherical inclusion. Predictions of the elastic constants of the composite are in good agreement with the experimental data avai lable in the literature. The influence of a weak interface has also been studied. The weak interface has the effect of debonding the filler particles from the matrix.

For the analysis of aligned discontinuous fiber composites an axisymmetric analysis was utilized since for spherical composites the results were in close 
agreement with the three-dimensional representation. An elastic analysis of a three phase (matrix, fiber and interface) fibrous composite was carried out to study in detail the role of an interface on the load carrying capacity of the composite. The relative importance of the various stress distributions and their influence on the strength of the composite has been discussed. It has been shown that by weakening the interface both the modulus of the composite and the strength of the composite can be independently altered. Thus it is possible to select an interface modulus to optimize the impact strength or energy absorption capability of the composite. The influence of an elastic-plastic matrix on internal stresses and composite properties was also studied. Here, different types of boundary conditions were applied to the axisymmetric cell representing the composite. It has been found that the behavior of a composite can be simulated very closely by carefully imposing the boundary conditions on the representative cell. The composite sttress-strain behavior has been predicted and a comparison has been made with available experimental data.

The present studies are an attempt to predict the behavior of complex composite materials using simplified micromechanics analyses. The powerful tool of finite element methods makes it possible to analyze complex problems with great ease. Limitations concerning the applicability of the results have been pointed out at the appropriate places in the thesis. Suggestions have been made for research work which would further enhance the understanding of the behavior of composite materials and would increase the confidence in the application of analyses such as the one presented here. 


\section{APPENDIX}

In case of elastic analysis it is possible to superpose specific individual boundary-value problems in order to obtain the solution for a given set of boundary conditions $(35,36)$. Superposition of two boundary value problems is needed for the axisymmetric analysis and three for the three-dimensional analysis.

\section{Axisymmetric Analysis}

To satisfy the boundary conditions stated in Section II, the following stress and displacement distributions (with reference to Fig. 1) were obtoined and properly superposed:

(1) The stress and displacement distribution (No. 1) is found such that

$$
\begin{aligned}
& \left(U_{z 1}\right)_{A B}=1 \text { (Arbitrarily specified unit displacement), } \\
& \left(U_{z 1}\right)_{D C}=0 \text { (Symmetry), } \\
& \left(U_{r 1}\right)_{B C}=0 \text { (Specified displacement condition), } \\
& \left(U_{r l}\right)_{A D}=0 \text { (Symmetry)! } \\
& \tau_{r z}=\tau_{z r}=0 \text { (on ABCD). }
\end{aligned}
$$

From these boundary conditions, $\sigma_{r 1}$ and $\sigma_{21}$ in all elements and displacements $U_{r 1}, U_{z 1}$ at all nodal circles are determined.

(2) The stress and displacement distribution (No. 2) is found such that

$$
\begin{aligned}
& \left(U_{z 2}\right)_{A B}=0 \text { (Specified displacement condition), } \\
& \left(U_{\mathrm{z} 2}\right)_{D C}=0 \text { (Symmetry), } \\
& \left(U_{\mathrm{r} 2}\right)_{B C}=1 \text { (Arbitrarily specified unit displacement), } \\
& \left(U_{\mathrm{r} 2}\right)_{A D}=0 \text { (Symmetry), } \\
& \tau_{r z}=\tau_{z r}=0 \text { (on ABCD). }
\end{aligned}
$$


142

(3) These stress and displacement distributions are superimposed to obtain

$$
\begin{aligned}
& \sigma=\sigma_{1}+k \sigma_{2} \\
& U=U_{1}+k U_{2}
\end{aligned}
$$

where $k$ is determined such that the net force in the $r$ direction along $B C$ is zero. Thus

$$
\left(F_{P_{B C}}=\int_{B C}\left(\sigma_{r 1}+k \sigma_{r 2}\right) d z=|B C|\left(\bar{\sigma}_{r 1}+k \bar{\sigma}_{r 2}\right)=0\right.
$$

(where $\bar{\sigma}_{\mathrm{p}}$ represents the average stress) so that

$$
\therefore k=-\left(\frac{\bar{\sigma}_{\mathrm{rl}}}{\bar{\sigma}_{\mathrm{r} 2}}\right)_{B C}
$$

The average stress on $A B$ is thus

$$
\left(\bar{\sigma}_{z}\right)_{A B}=\left(\bar{\sigma}_{z 1}\right)_{A B}-\left(\frac{\bar{\sigma}_{r 1}}{\delta_{r 2}}\right)_{B C}\left(\bar{\sigma}_{z 2}\right)_{A B}
$$

and the displacement is

$$
\begin{aligned}
\left(U_{z}\right)_{A B} & =\left(U_{z 1}\right)_{A B}-\left(\frac{\sigma_{r 1}}{\delta_{r 2}}\right)_{B C}\left(U_{z 2}\right)_{A B}=\left(U_{z 1}\right)_{A B} \\
\text { since }\left(U_{z 2}\right)_{A B} & =0 .
\end{aligned}
$$

To calculate the stiffness or modulus of elasticity of the composite, the average stress on the boundary $A B$ is calculated:

$$
\bar{\sigma}_{z}=\frac{\int_{A} \bar{\sigma}_{z} d A}{A}=\left(\bar{\sigma}_{z}\right)_{A B}
$$

where $A$ is the area of the top of the cylinder in the finite element analysis and the integral is replaced as a summation as follows:

$$
\int_{A} \sigma_{z} \mathrm{dA}=2 \pi \int_{0}^{r_{1}} \sigma_{z} r d r=2 \pi \sum_{i=1}^{n} \frac{3}{2}\left(r_{i}^{2}-r_{i-1}{ }^{2}\right) \sigma_{z}
$$


where $r_{i}$ and $r_{i-1}$ are the radii to the nodal circles that define the elements on the top of the cylinder, $n$ is the number of such circles, and $\sigma_{z}$ is the corresponding normal stress in each element.

The modulus is defined as

$$
E=\frac{\bar{\sigma}_{z}}{\bar{\varepsilon}_{z}}
$$

where the strain used is calculated from the specified boundary displacement,

$$
\vec{\varepsilon}_{z}=\frac{\left(U_{z}\right)_{A B}}{|B C|}
$$

$\left(U_{z}\right)_{A B}$ is the net displacement of boundary as follows:

$$
\left(U_{z}\right)_{A B}=\left(U_{z 1}\right)_{A B}+k\left(U_{z 2}\right)_{A B}
$$

but.

$$
\left(U_{22}\right)_{A B}=0
$$

thus

$$
\left(U_{z}\right)_{A B}=\left(U_{z}\right)_{A B}
$$

The displacement of the boundary BC is obtained in the some woy as

$$
\left(U_{B C}\right)_{B C}=\left(U_{B C}\right)_{B C}
$$

The net displacements of the boundaries $A B$ and $B C$ are used to calculate the Poisson's ratio which can be written as follows:

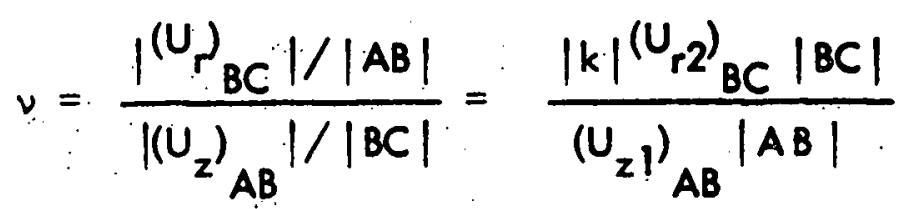

and since $\left(U_{r 2}\right)_{B C}=\left(U_{z 1}\right)_{A B}$ and $|B C|=|A B|$

$$
\nu=|k| \frac{|B C|}{|A B|}=|k|
$$


It may be observed that the modulus of elasticity and Poisson's ratio are calculated in the process of combining solutions.

Three-Dimensional Analysis

In the three-dimensional analysis, three solutions are needed to be superposed to get the desired boundary conditions as stated in the text. Otherwise, the procedure is quite similar to that for the axisymmetric analysis. The following steps are used with reference to Fig. 26

(1) The stress and displacement distribution (No. I) is found such that:

$$
\begin{aligned}
\left(U_{x l}\right)_{A B F E} & =1 \text { (Arbitrarily specified unit displacement), } \\
\left(U_{x}\right)_{D C G H} & =0 \text { (Symmetry), } \\
\left(U_{y !}\right)_{B C G F} & =0 \text { (Specified displacement condition) } \\
\left(U_{y 1}\right)_{A D H E} & =0 \text { (Symmetry), } \\
\left(U_{z l}\right)_{E F G H} & =0 \text { (Specified displacement condition), } \\
\left(U_{z 2}\right)_{A B C D} & =0 \text { (Symmetry) } \\
\text { and }{ }^{\top}{ }_{x y}{ }^{\top}{ }_{y z} & =T_{z x}=0 \text { (on all faces of the cube). }
\end{aligned}
$$

From these boundary conditions, stresses $\sigma_{x 1}, \sigma_{y l}$ and $\sigma_{z i}$ in all the elements and displacements $U_{x I}, U_{y l}$ and $U_{z l}$ at all the nodal points are determined.

(2) The stress and displacement distribution (No. 2) is found such that:

$$
\begin{aligned}
& \left(U_{x 2}\right)_{A B F E}=0 \text { (Specified displacement condition) } \\
& \left(U_{x 2}\right)_{D C G H}=0 \text { (Symmetry) } \\
& \left(U_{y 2}\right)_{B C G F}=1 \text { (Arbitrarily specified unit displacement) }
\end{aligned}
$$




$$
\begin{aligned}
&\left(U_{y 2}\right)_{A D H E}=0 \text { (Symmetry) } \\
&\left(U_{z 2}\right)_{E F G H}=0 \text { (Specified displacement condition) } \\
&\left(U_{z 2}\right)_{A B C D}=0 \text { (Symmetry) } \\
& \text { and } \tau_{x y}=\tau_{y z}=\tau_{z x}=0 \text { (on all faces of the cube). } \\
& \text { (3) The stress and displacement distribution (No. 3) is found such that: } \\
&\left(U_{x 3}\right)_{A B F E}=0 \text { (Specified displacement condition) } \\
&\left(U_{x 3}\right)_{D C G H}=0 \text { (Symmetry) } \\
&\left(U_{y 3}\right)_{B C G F}=0 \text { (Specified displacement condition) } \\
&\left(U_{y 3}\right)_{A D H E}=0 \text { (Symmetry) } \\
&\left(U_{z 3}\right)_{E F G H}=1 \text { (Arbitrarily specified unit displacement) } \\
&\left(U_{z 3}\right)_{A B C D}=0 \text { (Symmetry) } \\
& \text { and } \tau_{x y}={ }_{y z}^{T}=\tau_{z x}=0 \text { (on all faces of the cube). }
\end{aligned}
$$

(4) These stress and displacement distributions are superimposed to obtain:

$$
\begin{aligned}
& \sigma=k_{1} \sigma_{1}+k_{2} \sigma_{2}+\sigma_{3} \\
& u=k_{1} u_{1}+k_{2} u_{2}+u_{3}
\end{aligned}
$$

where $k_{1}$ and $k_{2}$ are determined such that net normal forces on the faces ABFE and $B C G F$ are zero. Thus

$$
\left(F_{x}\right)_{A B F E}=\int_{A B F E}\left(k_{1} \sigma_{x 1}+k_{2} \sigma_{x 2}+\sigma_{x 3}\right) d A=A\left(k_{1} \delta_{x 1}+k_{2} \sigma_{x 2}+\sigma_{x 3}\right)=0
$$

where $A$ is the area of face of the cube. Therefore $k_{1} \sigma_{x i}+k_{2} \sigma_{x 2}+\sigma_{\times 3}=0$. 
Summation of the force in $y$-direction on the face BCGF leads to another equation:

$$
k_{1} \delta_{y_{1}}+k_{2} \bar{\sigma}_{y_{2}}+\delta_{y^{3}}=0
$$

Solution of these equations gives

$$
k_{1}=-\frac{\bar{\sigma}_{y 2} \bar{\sigma}_{x 3}-\bar{\sigma}_{x 2} \bar{\sigma}_{y 3}}{\bar{\sigma}_{x 1} \bar{\sigma}_{y 2}-\bar{\sigma}_{y 1} \bar{\sigma}_{x 2}}
$$

and

$$
k_{2}=-\frac{\bar{\sigma}_{x 1} \bar{\sigma}_{y 3}-\bar{\sigma}_{y 1} \sigma_{x 3}}{\bar{\sigma}_{x 1} \bar{\sigma}_{y 2}-\bar{\sigma}_{y 1} \bar{\sigma}_{x 2}}
$$

The average normal stress on face EFGH is thus

$$
\left(\bar{\sigma}_{z}\right)_{E F G H}=\left(\bar{\sigma}_{z 3}\right)_{E F G H}+k_{1}\left(\bar{\sigma}_{z 1}\right)_{E F G H}+k_{2}\left(\bar{\sigma}_{z 2}\right)_{E F G H}
$$

Average stress, $\bar{\sigma}_{z}$, for a stress distribution is calculated as:

$$
\bar{\sigma}_{z}=\frac{\int_{A} \sigma_{z} d A}{A}
$$

where the integral is replaced by a summation as follows:

$$
\int_{A} \sigma_{z} d A=\sum_{i=1}^{n} \sigma_{z} A i
$$

where $\sigma_{z}$ is the stress in an element an'd $A_{i}$ the area of the element on the face under consideration and $n$ is the total number of such elements.

The modulus of elasticity is calculated from the average stress

$$
E=\frac{\bar{\sigma}_{z}}{\bar{\varepsilon}_{z}}
$$

where the strain used is calculated from the specified boundary displacement 


$$
\bar{\varepsilon}_{\mathbf{z}}=\frac{\left(\mathrm{U}_{\mathbf{z}}\right)_{E F G H}}{|A E|}
$$

$\left(U_{z}\right)_{E F G H}$ is the net normal displacement of the boundary as follows:

$$
\left(U_{z E F G H}=k_{1}\left(U_{z 1}\right)_{E F G H}+k_{2}\left(U_{z 2}\right)_{E F G H}+\left(U_{z 3}\right)_{E F G H}\right.
$$

but

$$
\left(U_{Z 1}\right)_{E F G H}=\left(U_{Z 2}\right)_{E F G H}=0
$$

thus

$$
\left(U_{z E F H}\right)=\left(U_{23}\right)_{E F H}
$$

The normal displacement of the faces $A B F E$ and $B C G F$ are found to be:

$$
\begin{aligned}
& \left(U_{x 1}\right)_{A B F E}=k_{1}\left(U_{x 1}\right)_{A B F E} \\
& \left(U_{Y}\right)_{B C G E}=k_{2}\left(U_{y 2}\right)_{B C G E}
\end{aligned}
$$

Poisson's ratios $v_{z x}$ and $v_{z y}$ are calculated from these displacements:

$$
\nu_{z x}=\frac{\left|\left(U_{x}\right)_{A B F E}\right| /|A D|}{\left|\left(U_{z}\right)_{E F G H} V\right| A E \mid}=\frac{\left|k_{1}\right|\left(U_{x \mid}\right)_{A B F E}|A E|}{\left(U_{z 3}\right)_{E F G H}|A D|}
$$

$$
\begin{aligned}
\text { since }\left(U_{X 1}\right)_{A B F E} & =\left(U_{z 3}\right)_{E F G H} \text { and }\left|A E_{1}\right|=|A D| \\
v_{z X} & =k_{1}
\end{aligned}
$$

Similarly

$$
v_{z y}=k_{2}
$$

Due to the symmetry of the problem $v_{z x}$ and $v_{z y}$ should be the same. However, the size and the orientation of the elements near the surface may cause some difference in the values of $k_{1}$ and $k_{2}$. Here also the modulus of elasticity and Poisson's ratio are calculated in the process of combining the solutions. 


\section{BIBLIOGRAPHY}

1. Goodier, J. N., "Concentrations of Stress Around Spherical and Cylindrical Inclusions and Flaws," J. Appl. Mech., 1 (1) 39-44; 1933.

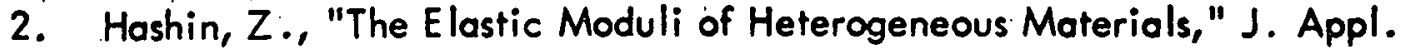
Mech., 29, 143, 1962.

3. Hashin, Z. and Shirikman, S., "Variational Approach to the Theory of Elastic Behavior of Multiphase Materials," J. Mech. Phys. Solids, 11 (2) 127-40, 1963.

4. Hashin, Z: "Theory of Mechanical Behavior of Heterogeneous Media," Appl. Mech., Rev. 17, 1, 1964.

5. Hill, R., "Elastic Properties of Reinforced Solids: Some Theoretical Principles," J. Mech. Phys. Solids, 11, 357, 1963.

6. Holister, G. S. and Thomas, C., Fiber Reinforced Materials, Elsevier Publishing Company, London, 1966.

7. Muskhelishvili, Some Basic Problems of the Mathematical Theory of Elasticity, (translated by J. R. M. Radok), Erven P. Noordhoff, N. V., Gronningen, Netherlands, 1963.

8. Zienkiewicz, O.C., The Finite Element Method, McGrow-Hill Publishing Co., Ltd., London, 1967.

9. Hasselman; D.P.H. and Fulrath, R. M., "Micromechanical Stress Concentrations in Two Phase Brittle-Matrix Ceramic Composites," J. Am. Ceram. Soc., 50 (8) 399-404, 1967.

10. Bertolotti, R. L. and Fulrath, R. M., "Effect of Micromechanical Stress Concentrations on Strength of Porous Glass," J. Am. Ceram. Soc. 50 (II) $558-62,1967$.

11. Stett, M. A. and Fulrath, R. M., "Mechanical Properties and Fracture Behovior of Chemically Bonded Composites," J. Am. Ceram. Soc., 53 (1) $5-13,1970$.

12. Davidge, R. W. and Green, T. J., "The Strength of Two-Phase Ceramic/ Glass Materials," J. Materials Science, 3, 629-34, 1968.

13. Wilson, E. L., Structural Engineering Laboratory Report 63-1, University of California, Berkley, California, June 1963.

14. Hasse Iman, D.P.H. and Fulrath, R. M., "Effect of Alumina Dispersion on Young's Modulus of a Glass," J. Am. Ceram. Soc. 48 (4) 218-19, 1965. 
15. Hasselman, D.P.H. and Fulrath, R. M., "Effect of Spehrical Tungsten Dispersion on Young's Modulus of a Glass," J. Am. Ceram. Soc. 48 (10), 548-49, 1965.

16. Broutman, L..J. and Panizza, G., "Micromechanics Studies of Rubber Reinforced Glassy Polymers," Int. J. Poly. Mat., Vol. I, 95-109, 1971.

17. Fryxell, R. E. and Chandler, B. A., "Creep, Strength, Expansion and Elastic Moduli of Sintered BeO as a Function of Grain Size, Porosity, and Grain Orientation," J. Am. Ceram. Soc. 47 (6) 283-91, 1964.

18. Manning, W. R., Hunter, Jr., O., and Powell, Jr., B. R., "Elastic Properties of Polycrystalline Yttrium Oxide, Dysprosium Oxide, Holmium Oxide, and Erbium Oxide: Room Temperature Measurements," J. Am. Ceram. Soc. 52 (8) 436-42, 1969.

19. Cornell, D. C., Jadhov, K. B. and Rashid, Y. R., "SAFE-3D-A Computer Program for the Three-Dimensional Stress Analysis of Composite Structures," General Dynamics, AEC Research and Development Report, GA-7855, September 1; 1967.

20. Cox, H. L., "The Elasticity and Strength of Paper and Other Fibrous Materials," British J. Appl. Phys., Vol. 3, 72, 1952.

21. Dow, N. F., "Study of Stress Near a Discontinuity in a Filament Reinforced Composite Material," G.E.C., Missile and Space Division Report No. R63SD61, 1963.

22. Rosen, B. W., "Mechanics of Fiber Strengthening," Fiber Composite Materials, Chapter 3, ASM Publication for Seminar of ASM, October 1960.

23. Tyson, W. R. and Davies, G. J., "A Photoelastic Study of the Shear Stresses Associated with the Transfer of Stress During Fiber Reinforcement," British J. Appl. Phys., Vol. 16, 199, 1965.

24. Schuster, D. M. and Scala, E., "The Mechanical Interaction of Saphire Whiskers with a Birefringent Matrix," Trans. Met. Soc. of AIME, Vol. 230, p. 1635, Dec. 1964.

25. Fujiwara, M., "Resin-Fiber Load Transfer in Fiber-Reinforced Plastics," MIT Research Report R67-8, February 1967.

26. Carrara, A. S. and McGarry, "Matrix and Interface Stresses in a Discontinuous Fiber Composite Model," J. Comp. Mat., 2 (2), 222-243, April 1968.

27. Maclaughlin, T. F. and Barker, R. M., "Effect of Modulus Ratio on Stress Near a Discontinuous Fiber," Experimental Mechanics, 12 (4), 178-183, April 1972.

28. Broutman, L. J. and Krock, R. H., Modern Composite Materials, AddisonWesley Publishing Co., Reading, Mass. 1967. 
29. Ashton, J. E., Halpin, J. C., and P. H. Petit, Primer on Composite Materials: Analysis, Technomic Publishing Co., Inc., Stamford, Conn., 1969.

30. Chen, P. E. and Lovengood, R. E.., "Stress Field 'Around Multiple Inclusions," Proc. Southampton 1969, Civil Engineering Materials Conference, Part I, 75-86, April 1969.

31. Doughty, C. B. and McGarry, F. J., "Viscoelastic and Elastic-Plastic Analysis of a Fiber Reinforced Composite Material," MIT Research Report R68-89, December 1968.

32. Adams, D. F., "Inelastic Analysis of a Unidirectional Composite Subjected to Transverse Normal Loading," J. Comp. Mat., Vol. 4, 310-328, July 1970.

33. Kelly, A. and Tyson, W. R., "Tensile Properties of Fiber-Reinforced Metals: Copper/Tungsten and Copper/Molybdenum," J. Mech. Phys. Solids, Vol. $13,329-350,1965$.

34. Weeton, J. W. and Signorelli, R. A., "Fiber Metal Composites," Proc. 12th Sagamore Army Materials Research Conference, 477-530, Aug. 1965.

35. Chen, P. E. and Lin, J. M., "Transverse Properties of Fibrous Composites," Mat. Res. Stand., 9 (8) 29-33, 1969.

36. Adams, D. F. and Doner, D. R., "Transverse Normal Loading of a Unidirectional Composite," J. Comp. Mat. I (2) 152-164, April 1967. 